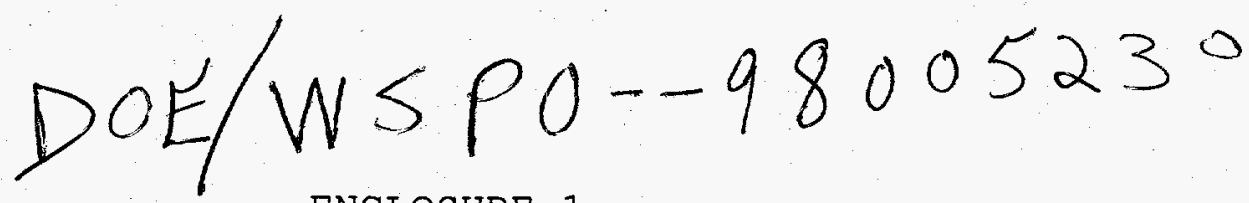

ENCLOSURE 1

\title{
QUARTERIY ENVIRONMENTAL DATA SUMMARY FOR FIRST QUARTER 1998
}

In support of the Weldon Spring Site Remedial Action Project Federal Facilities Agreement, a copy of the Quarterly Environmental Data Summary (QEDS) for the first quarter of 1998 is enclosed.

The data presented in this letter and attachment constitute the QEDS. The data were received from the contract laboratories, verified by the Weldon Spring Site verification group and, except for air monitoring data and site KPA generated data (uranium analyses), merged into the data base during the first quarter of 1998. Air monitoring data presented are the most recent complete sets of quarterly data. Air data are not stored in the data base, and KPA data are not merged into the regular data base.

Significant data, defined as data values that have exceeded defined "above normal" Level 2 values, are discussed in this letter for Environmental Monitoring Plan (EMP) generated data only. Above normal Level 2 values are based, in ES\&H procedures, on historical high values, DOE Derived Concentration Guides (DCGs), NPDES limits and other guidelines. The procedures also establish actions to be taken in the event that "above normal" data occur.

All data received and verified during the first quarter were within a permissible range of variability except for those detailed below. Above normal occurrences are cited for groundwater, air, and NPDES data. There were none for springs or surface water. The following discussion offers a brief summary of the data merged during the first quarter that exceeded the above normal criteria and updates on past reported above normal. data. The attached tables present the most recent data for air and the data merged into the data base during the first quarter 1998 for groundwater, NPDES, surface water, and springs.

Graphs showing concentrations of selected contaminants of concern at some of the critical locations have also been included in this QEDS. The graphs are discussed in the separate sections.

\section{NPDES}

The uranium concentrations at the three major NPDES outfalls are graphed for January 1997 to March 1998. The graphs are located at the beginning of the NPDES tables. For comparison purposes, the annual average for 1997 is also graphed. The derived concentration guide (DCG) of $600 \mathrm{pCi} / 1$ for uranium may also be used for comparison. The DCG is the concentration of a radionuclide in air or water that, under conditions of continuous exposure for one year by one exposure mode (i.e., ingestion of water), would result in an effective dose equivalent of $100 \mathrm{mrem}$. A graph of historical annual averages fpr the three outfalls is also attached. 


\section{DISCLAIMER}

Portions of this document may be illegible electronic image products. Images are produced from the best available original document. 


\section{PAGE 2: QUARTERIY ENVIRONMENTAL DATA SUMMARY FOR FIRST QUARTER 1998}

Outfall NP-0002 was slightly elevated above the 1997 annual average for uranium (14 pCi/l) for each of the three months of the quarter. The values were still well below the DCG and are suspected to be natural variations. The majority of the NP-0002 watershed has been remediated, and uranium levels are expected to stay well below the pre-remediation levels.

The uranium levels at NP-0003 were slightly lower than the 1997 annual average (143 pCi/l) for the quarter. The final 1997 annual average was $143 \mathrm{pCi} / 1$ (an increase over the 1996 annual average of $88 \mathrm{pCi} / \mathrm{ll}$. While most of the Outfall NP-0003 watershed has been remediated, it contains Ash Pond, where contaminated soils, concrete, etc., are being stored. This has probably contributed to the higher levels at NP-0003 for 1997 and early 1998. Water is released from the Ash Pond area only if it is less than $600 \mathrm{pCi} / 1$; however, it may still be higher than the previous Outfall NP-0003 annual average which could cause an increase in the current annual average. The volume of runoff from Ash Pond is low in comparison to the remainder of the watershed. An upward trend for uranium is not expected because the surface area exposed to storm water is not expected to increase. Stabilization of soil piles stored in the Ash Pond area will likely help reduce uranium levels in the storm water run off. As material is removed for placement in the cell, fluctuating uranium levels are expected.

outfall NP-0005 was below the 1997 annual average for uranium (19 pCi/1) for January and March and above for February. The slightly elevated level is suspected to be a natural variation. A large portion of the NP-0005 watershed has been remediated, and uranium levels are expected to remain well below pre-remediation levels.

Outfall NP-0010, the CMSA sedimentation basin outfall, remained below the 1997 annual average $(2.7 \mathrm{pCi} / \mathrm{l})$ for January and February for uranium. For March uranium was greater than the 1997 annual average but still much less than the DCG of 600 $\mathrm{pCi} / 1$. The area has been remediated and uranium levels are expected to remain low, but the future use of a small area for vehicle maintenance may increase uranium levels slightly. Because NP-0010 is a minor outfall it is not graphed.

The above normals for data merged during the first quarter and any above normals that were not resolved in previous QEDS are discussed below.

\section{NPDES HYDROSTATIC TEST WATER}

There were four Level 2 above normals for the first quarter 1998 . The above normals occurred for hydrostatic test water released at the Chemical stabilization and Solidification (CSS) facility. potable water is used for the tests. The four occurrences are listed below. Two occurrences were from one sample. 


\section{PAGE 3: QUARTERIY ENVIRONMENTAI DATA SUMMARY FOR FIRST QUARTER 1998}

The water from these discharges flows to sedimentation Basin 4 and then to Outfall NP-0003. There was no known effect on the receiving stream and compliance has been met after corrective actions were taken.

$\mathrm{NP}-\mathrm{CSSH}-020998$

The TSS for this sample was $140 \mathrm{mg} / 1$, and the NPDES limit is 100 $\mathrm{mg} / \mathrm{l}$. The $\mathrm{pH}$ was 9.08 and the permissible range is 6.0 to 9.0 . The cause for the elevated levels is not definitely known, but the elevated TSS is believed to be the result of soil in the pipe being tested, and the $\mathrm{pH}$ is believed to have been caused from a high $\mathrm{pH}$ in the influent potable water.

NP-CSSH-030698

The TSS for this sample was $113 \mathrm{mg} / \mathrm{l}$, which is above the NPDES limit of $100 \mathrm{mg} / \mathrm{l}$. The elevated level is believed to be the result of allowing the water to accumulate in a sump prior to discharge. The sump has a rock bottom and the water was drawn from near the bottom during the pumping operation.

NP-CSSH-031198

The TSS for this sample was $141 \mathrm{mg} / \mathrm{l}$, which is above the NPDES limit of $100 \mathrm{mg} / \mathrm{l}$. The elevated level was again the result of pumping from a rock lined sump. When the results were received on March 16 for the sample collected on March 6, 1998, the hose was elevated above the bottom of the sump. One final discharge was made from the sump on March 18, and all parameters were in compliance with the permitted limits. Use of the sump was discontinued after the March 18 sample.

\section{GROUNDTATER}

\section{Chemical Plant}

\section{Site Water Treatment Plant and Temporary Storage Area}

Reported concentrations of metals continue to occasionally exceed baseline values. The majority of these are attributable to comparing new data from unfiltered samples to baseline values which were established using filtered sample data. During the first quarter of 1998, baseline values were re-evaluated and adjusted to compensate for differences between filtered and unfiltered samples. Beginning in the second quarter of 1998, analytical results which exceed original baseline values will be compared to the adjusted values. If the reported value does not exceed the adjusted baseline, the sample will not be reported as above normal. 


\section{PAGE 4: QUARTERIY ENVIRONMENTAL DATA SUMMARY FOR FIRST QUARTER 1998}

GW-2036-Q397, GW-2036-Q497, and GW-2036-Q198

The third quarter 1997 arsenic concentration (4.2 ug/1) at this monitoring location along the west side of the Temporary storage Area (TSA) was above baseline $(2.09 \mathrm{ug} / 1)$. The subsequent fourth quarter 1997 and first quarter 1998 analytical results were reported as below detection limits, suggesting that the third quarter value was anomalous and that the groundwater does not have elevated arsenic concentrations at this location.

$\mathrm{GW}-2038-\mathrm{Q} 397, \mathrm{GW}-2038-\mathrm{Q} 497$, and $\mathrm{GW}-2038-\mathrm{Q} 198$

The third quarter 1997 lead concentration (17.7 ug/1) for this monitoring location south of Raffinate Pit 3 was above the baseline concentration $(1.65 \mathrm{ug} / 1)$. The subsequent fourth quarter 1997 and first quarter 1998 analytical results were reported as below detection limits, suggesting that the third quarter value was anomalous and that the groundwater does not have elevated lead concentrations at this location.

GW-2039-Q397, GW-2039-Q397-RE and GW-2039-Q497

Arsenic for the third quarter 1997 (107 ug/l) was reported as approximately two orders of magnitude above baseline (2.43 ug/1) for this location along the east side of the TSA. A laboratory reporting error was suspected, and the sample was reanalyzed at an alternate lab (GW-2039-Q397-RE). The reanalyzed sample and the fourth quarter 1997 sample (GW-2039-Q497) were reported with no detection of arsenic, suggesting that the $107 \mathrm{ug} / \mathrm{I}$ value was in error.

GW-2041-Q397, GW-2041-Q497, and GW-2041-Q198

The third quarter arsenic concentration (5.5 ug/l) for this location west of the Site Water Treatment Plant equalization basin was reported above baseline $(4.35 \mathrm{ug} / 1)$. The fourth quarter 1997 and first quarter 1998 samples (GW-2041-Q497 and GW2041-Q198, respectively) had no arsenic detections, suggesting that there are no elevated arsenic levels at this location.

GW-2043-Q497 and GW-2043-Q198

The fourth quarter nitrate value $(494 \mathrm{mg} / 1)$ was reported as two orders of magnitude above baseline $(8.03 \mathrm{mg} / 1)$ for this location east of the Site Water Treatment Plant equalization basin. The subject sample value could not be validated, and a subsequent sample (GW-2043-Q198) was reported within normal range (6.83 $\mathrm{mg} / \mathrm{ll}$. This subsequent value suggests that the original $494 \mathrm{mg} / \mathrm{l}$ value was in error. 
PAGE 5: QUARTERIY ENVIRONMENTAL DATA SUMMARY FOR FIRST QUARTER 1998

\section{VoC Monitoring}

Monthly monitoring for the Volatile Organic Compounds (VOC's), specifically trichloroethene(TCE), south and west of Raffinate Pits 3 and 4 at WSSRAP locations MW-2037, MW-2038, MW-3025 and Department of Army location MW-S02I continued throughout 1997. During the first quarter 1998, monitoring frequency was reduced to once every two months for the same locations. The reduced monitoring frequency was justified by the relatively slow TCE concentration changes observed during 1996 and 1997.

GW-2037-1197, GW-2037-1297, GW-2037-B197, GW-2038-1197, GW2038-1297, GW-2038-B198, GW-3025-1197, GW-3025-1297, GW-3025B198, MW-S004-B198, MW-S021-1197, MW-S021-1297, and MW-S021B198

First quarter 1998 TCE concentrations at all locations remained approximately the same as those measured in late 1997. A slight decrease was reported for location MW-S021. The TCE concentrations at these locations are plotted versus time on the attached figure. Bi-monthly sampling is scheduled to continue through 1998, and the results will be reported in subsequent QEDS.

TCE was detected above quantification levels for the first time at location MW-S004 during the first quarter of 1997. TCE at this location was reported at a concentration of $21 \mathrm{ug} / 1$.

\section{Enhanced Raffinate Pit Area Monitoring}

Enhanced raffinate pit area groundwater monitoring for selected locations in the southern and western portions of the site is intended to provide increased capability of detecting effects on groundwater from disturbance of raffinate pit sludge and soil during ongoing remediation efforts. The monitoring includes bimonthly sampling of 11 groundwater monitoring locations (MW2037, MW-2038, MW-2039, MW-3003, MW-3023, MW-3024, MW-3025, MW3027, MW-4001, MW-4002, and MW-4006). The analytical parameters for the 11 locations include nitrate, sulfate, metals (Toxicity List), and total uranium (on-site KPA).

$\mathrm{GW}-3027-1297$

An above normal nitrate concentration (453 $\mathrm{mg} / \mathrm{l}$ ) was reported for the December 1997 sample at this location southwest of Raffinate Pit 4. The historical nitrate mean concentration at this location is $55.6 \mathrm{mg} / \mathrm{l}$. It is possible that the increased concentration reflects a localized mobilization of raffinate pit impacted groundwater resulting from remediation activities. No other locations or contaminant parameters were apparently impacted. Subsequent data from this location will be used to 


\section{PAGE 6: QUARTERIY ENVIRONMENTAL DATA SUMMARY FOR FIRST QUARTER 1998}

assess the source of this elevated nitrate value.

\section{Disposal Cell Detection Monitoring}

Groundwater sampling for the first quarter 1998 Disposal Cell Detection Monitoring System (MW-2032, MW-2045, MW-2046, MW-2047, and MW-2048) was completed in January, 1998. The analytical results from this event were within the expected ranges (based upon the previous year's sampling events) for all parameters.

The sampling results from this baseline monitoring are statistically evaluated quarterly and these evaluations are being used to determine baseline groundwater quality conditions against which subsequent monitoring results (during and after waste placement) will be compared.

\section{WELDON SPRING QUARRY}

\section{Quarry Water Treatment Plant}

Chloride and fluoride levels for groundwater monitoring locations in the vicinity of the Quarry Water Treatment Plant remain above baseline. St. Charles County began fluoridating the public water supply in June 1997. It was suspected that the recent fluoride and chloride increases, and previous levels of chloride (and related elevated metals) were related to a subsurface release of county water from water main lines located along Missouri State Highway 94. The PMC coordinated a field inspection and water line pressure test with St. Charles County Public water Supply District Number 2 to investigate this possibility. No leaks along the water lines were detected. Other than road salts used along Missouri Route 94 adjacent to monitoring well MW-1035 and use of hydrochloric acid at the quarry for decontamination purposes (which ended in 1995), no other sources of chlorides have been discovered. Due to the continued presence of elevated chloride and fluoride, an external source related to treated public water lines is suspected.

It is suspected that apparent elevated metals concentrations are related to comparing unfiltered sample results to baseline values established using filtered sample data. During the first quarter of 1998, baseline values were re-evaluated and adjusted to compensate for differences between filtered and unfiltered samples. Beginning in the second quarter of 1998, analytical results which exceed original baseline values will be compared to the adjusted values. If the reported value does not exceed the adjusted baseline, the sample will not be reported as above normal. 


\section{PAGE 7: QUARTERLY ENVIRONMENTAL DATA SUMMARY FOR FIRST}

GW-1035-Q198, GW-1036-Q198, and GW-1040-Q497

Chloride concentrations at the above-listed monitoring locations exceeded baseline values during the fourth quarter 1997 and the first quarter 1998. Since the second quarter 1997, chloride levels have decreased at all locations: $M W-1035$ from $39.7 \mathrm{mg} / 1$ to $19 \mathrm{mg} / \mathrm{l}$ (baseline is $14 \mathrm{mg} / \mathrm{l}$ ); $\mathrm{MW}-1036$ from $130 \mathrm{mg} / \mathrm{l}$ to $106 \mathrm{mg} / \mathrm{l}$ (baseline is $102 \mathrm{mg} / \mathrm{l}$ ); and MW-1040 from $33.9 \mathrm{mg} / 1$ to $19.1 \mathrm{mg} / 1$ (baseline is $16 \mathrm{mg} / 1$ ). Temporal similarities common to these locations include increases during 1996 followed by decreases in approaching baseline conditions beginning in second quarter, 1997. These similarities suggest a common chloride source for this groundwater impact. However, spatial distribution of the elevated chloride does not support this. Groundwater at MW-1041, approximately mid-way between locations MW-1036 and MW1040, had no elevated chloride levels to any noticeable degree during this period. Location MW-1037, hydraulically. downgradient from MW-1040, was also not affected. Considering the decreasing concentrations reported for the last four quarterly monitoring periods, it is expected that chloride concentrations will approach baseline levels in samples collected later in 1998 .

GW-1036-Q497, GW-1036-Q497-F, GW-1037-Q497, $\mathrm{GW}-1037-\mathrm{Q} 497-\mathrm{F}$, and $\mathrm{GW}-1037-\mathrm{Q} 198$

Metal concentrations elevated above baselines were reported in two locations along the east side of the Quarry Water Treatment Plant. During the fourth quarter 1997, lead concentrations at $\mathrm{MW}-1036$ (2.3 ug/1) and $\mathrm{MW}-1037$ (17.4 ug/l) were elevated above baselines $(2.06 \mathrm{ug} / 1$ and $2.30 \mathrm{ug} / 1$, respectively). Filtered replicates were collected during the fourth quarter sampling event. Lead concentrations at both locations in the filtered samples were below their respective baseline values (MW-1036 lead was not detected and $M W-1037$ was $2.2 \mathrm{ug} / 1$ ). Chromium at $\mathrm{MW}-1037$ $(9.3 \mathrm{ug} / 1)$ was also above baseline $(7.57 \mathrm{ug} / 1)$ for the fourth quarter of 1997. The filtered replicate for this parameter was also below baseline (below the detection limit of $2.0 \mathrm{ug} / 1$ ). These results demonstrate the inconsistencies involved in comparing unfiltered sample data to baselines established from filtered sample results, and support the proposed comparisons to adjusted baselines for all subsequent data review.

\section{Quarry Vicinity}

Groundwater quality in the quarry vicinity continues to show improvement at locations along the quarry rim for total uranium and nitroaromatic compounds. Total uranium activity in groundwater monitoring locations along the quarry rim decreased approximately 11\% during calendar year 1997. The nitroaromatic compounds TNT and DNT decreased approximately $40 \%$ and 18\%, respectively, at these locations during 1997. 


\section{PAGE 8: QUARTERLY ENVIRONMENTAL DATA SUMMARY FOR FIRST QUARTER 1998}

No above-normal contaminant concentrations were reported for the fourth quarter 1997 or first quarter 1998 data.

\section{St. Charles County Well Field}

No elevated levels of contaminants originating from the Weldon Spring Quarry were reported during the first quarter 1998 data review. Uranium activity results from the last four sampling events at the st. Charles county Well Field are shown on the attached figure

\section{SPRINGS}

No elevated contaminants were reported in samples from springs during the first quarter of 1998.

\section{SURFACE WATER}

No surface water contaminants (at Level 2 above normal concentrations) were detected in samples collected during the first quarter of 1998.

\section{AIR}

Data for the monitoring locations are attached. Graphs of the monitoring results are located in front of the tables and discussed below. There was one above normal air monitoring result for the fourth quarter of 1997.

The gross alpha concentrations for air particulate monitoring for the first quarter 1997 are graphed with the background level, which is based on the second quarter 1996 to first quarter 1998 average concentration, and background +3 standard deviations shown for comparison. The background location is AP-4012, at the Daniel Boone Elementary School in New Melle.

The gamma exposure from environmental TLD monitoring results for the fourth quarter 1998 are graphed with the background and the background plus 25 mrem quarterly committed effected dose equivalent ( $C E D E$ ) shown for comparison. The background level is based on monitoring conducted from the first quarter 1997 to the fourth quarter of 1997. The 25 mrem CEDE is based on one-fourth of the annual 100 mrem CEDE established in DOE Order 5400.5. The background locations are TD-4005 (west of the Army site) and TD4009 (Daniel Boone Elementary School, New Melle).

The alpha track radon and thoron monitoring results are graphed for the fourth quarter of 1997. The background level (based on 1997 monitoring) and the Derived Concentration Guide (DCG) for radon and thoron are shown for comparison. The DCG is a reference value for protection of the public and the environment contained in DOE Order 5400.5. Although some locations had concentrations above the DCG, they are all within the site boundary. The background locations are RD-4005 (west of the Army 


\section{PAGE 9: QUARTERLY ENVIRONMENTAL DATA SUMMARY FOR FIRST}

QUARTER 1998

site) and RD-4009 (Daniel Boone Elementary School, New Melle). One above normal for air monitoring that occurred during the fourth quarter is discussed below.

\section{RAFFINATE PIT 4 AND TSA}

$\underline{R D-3003-0497}$

During the fourth quarter 1997, the average thoron concentration measured at the alpha-track monitoring station located just to the west of the TSA and south of Raffinate Pit 4 (RD-3003) was $1.1 \mathrm{pCi} / 1$. This level was $1.0 \mathrm{pCi} / 1$ above the background concentration of $0.1 \mathrm{pCi} / 1$, and was therefore considered an "above normal" for a site perimeter monitoring location. This elevated concentration was attributed to thoron-generating waste

stored at the TSA and remedial activities conducted in Raffinate Pit 4. A dose assessment based on the elevated thoron concentration at $\mathrm{RD}-3003$ indicated a resulting committed effective dose equivalent (CEDE) of less than 0.1 mrem to a nearby member of the public. The site perimeter in the vicinity of the raffinate pits continues to be monitored for radon and thoron by both alpha-track and electret monitors.

\section{SUMMARY}

The previously described data are highlighted as being above prescribed baseline values, varying from historical ranges or being above regulatory limits, and as a result, are subject to more focused attention by the WSSRAP Environmental Protection Group. Continuous trends are monitored to determine the need for additional possible action. Except for the highlighted data, all other indicators subject to reporting in the QEDS were within historic range or below reporting criteria. 


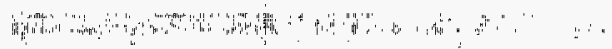

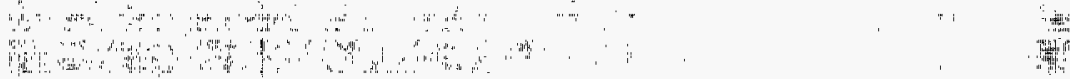

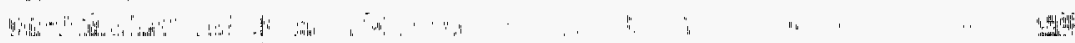

(1)

and

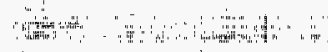

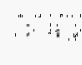

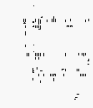

$\cdots$
$\cdots$
$\cdots$

inting

:

L.

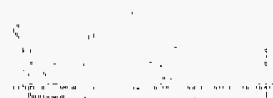

. 


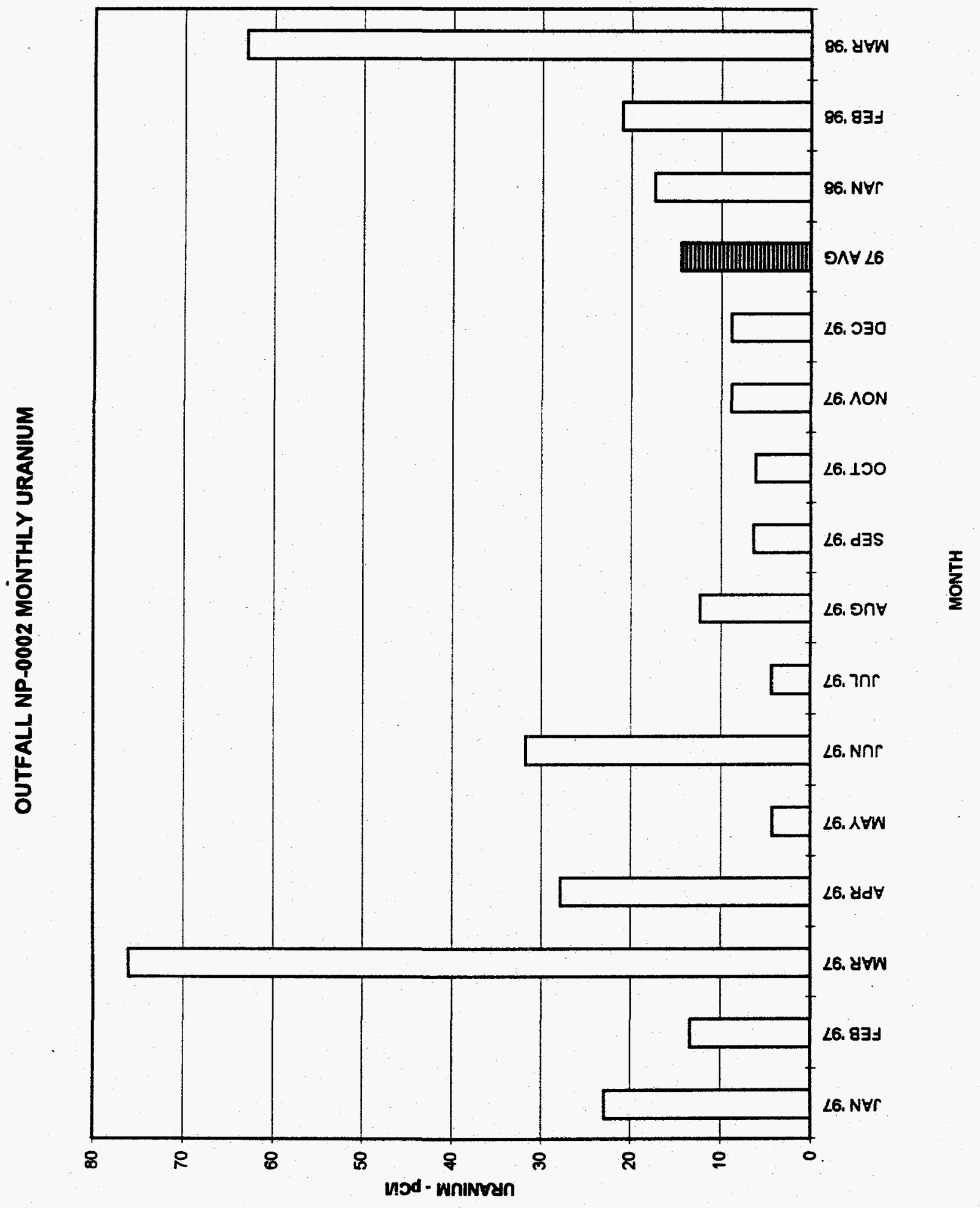

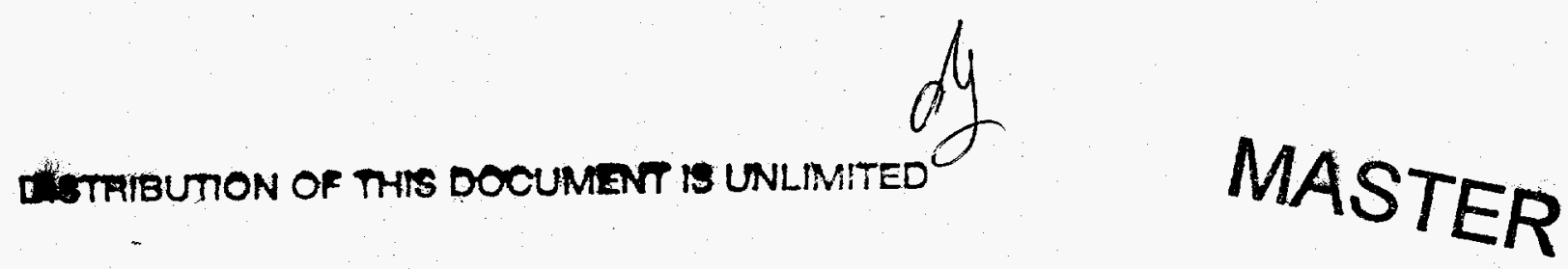




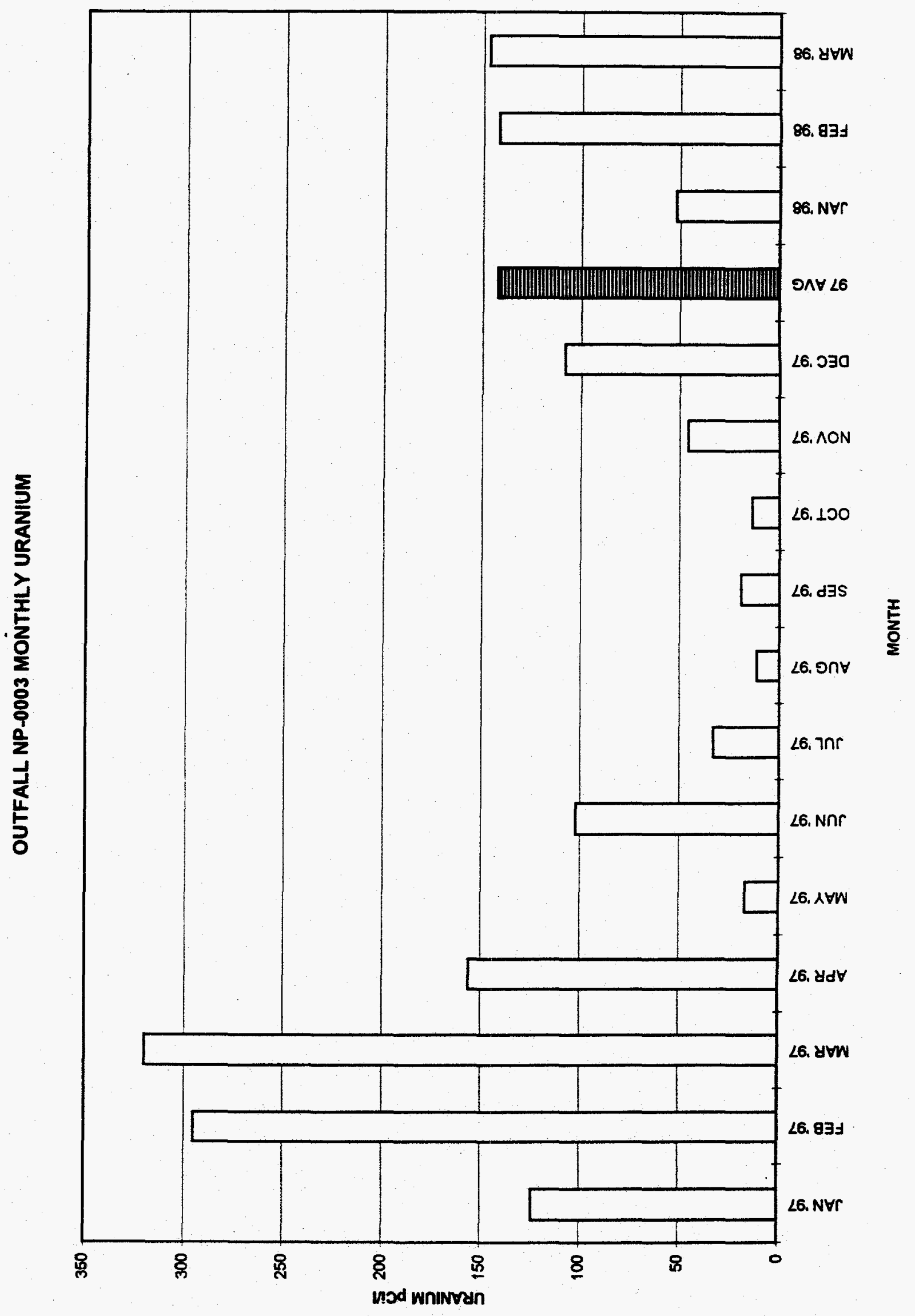




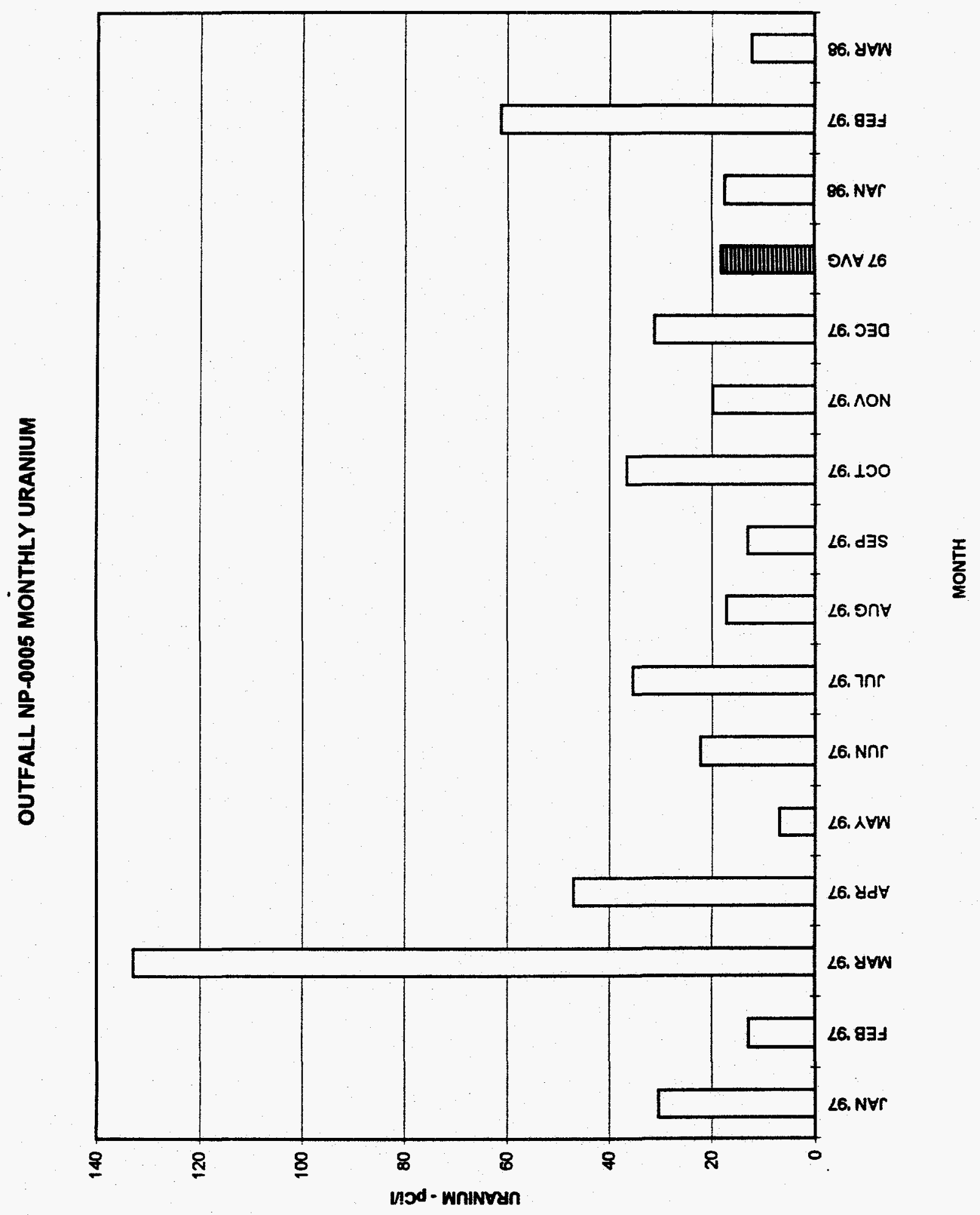




\section{URANIUM ANNUAL AVERAGE}

MAJOR NPDES STORM WATER OUTFALLS

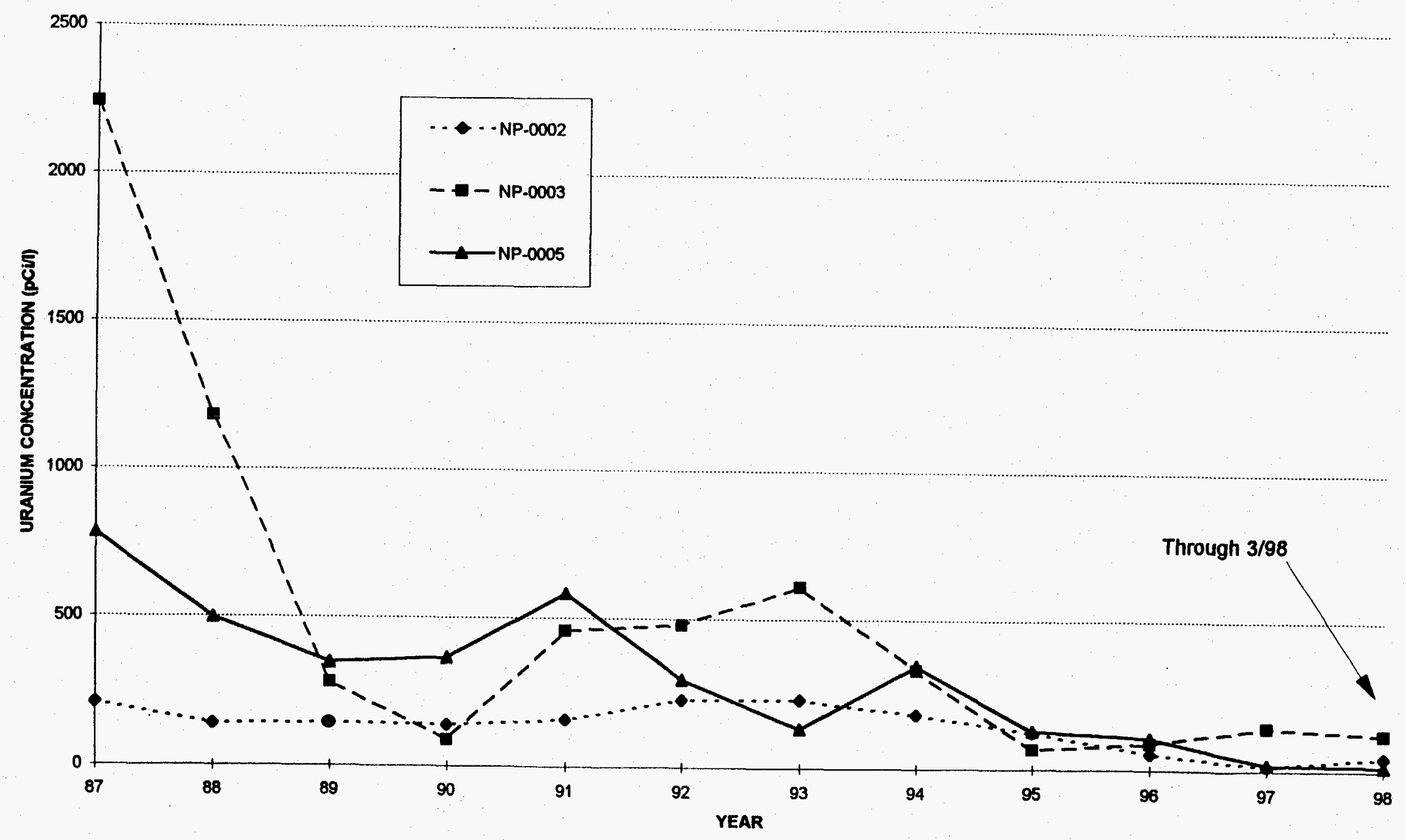


MPDES

DATA MERGED DURING FIRST QUARTER 1998

\begin{tabular}{|c|c|c|c|c|c|c|}
\hline WSSRAP_ID & DATE_SAM & PARAMETER & CONC & DL & UNITS & METHOO \\
\hline 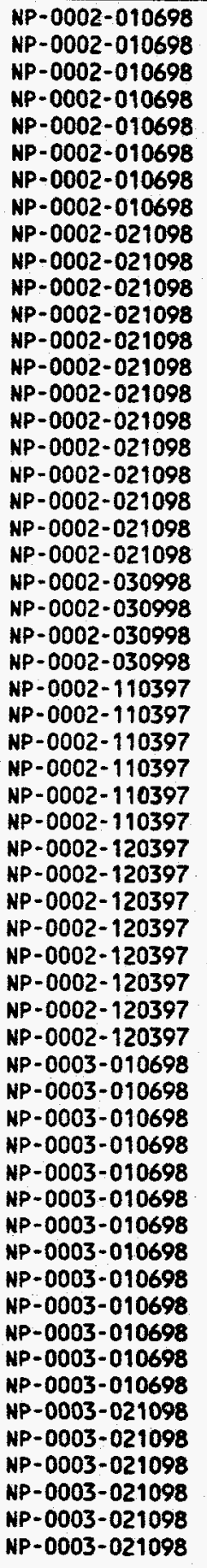 & $\begin{array}{l}01 / 06 / 98 \\
01 / 06 / 98 \\
01 / 06 / 98 \\
01 / 06 / 98 \\
01 / 06 / 98 \\
01 / 06 / 98 \\
01 / 06 / 98 \\
01 / 06 / 98 \\
02 / 10 / 98 \\
02 / 10 / 98 \\
02 / 10 / 98 \\
02 / 10 / 98 \\
02 / 10 / 98 \\
02 / 10 / 98 \\
02 / 10 / 98 \\
02 / 10 / 98 \\
02 / 10 / 98 \\
02 / 10 / 98 \\
02 / 10 / 98 \\
02 / 10 / 98 \\
02 / 10 / 98 \\
03 / 09 / 98 \\
03 / 09 / 98 \\
03 / 09 / 98 \\
03 / 09 / 98 \\
11 / 03 / 97 \\
11 / 03 / 97 \\
11 / 03 / 97 \\
11103 / 97 \\
11 / 03 / 97 \\
11 / 03 / 97 \\
12 / 03 / 97 \\
12 / 03 / 97 \\
12 / 03 / 97 \\
12 / 03 / 97 \\
12 / 03 / 97 \\
12 / 03 / 97 \\
12 / 03 / 97 \\
12 / 03 / 97 \\
01 / 06 / 98 \\
01 / 06 / 98 \\
011 / 06 / 98 \\
01 / 06 / 98 \\
01 / 06 / 98 \\
01 / 06 / 98 \\
01 / 06 / 98 \\
01 / 06 / 98 \\
01 / 06 / 98 \\
01 / 06 / 98 \\
01 / 06 / 98 \\
01 / 06 / 98 \\
01 / 06 / 98 \\
02 / 10 / 98 \\
02 / 10 / 98 \\
02 / 10 / 98 \\
02 / 10 / 98 \\
02 / 10 / 98 \\
02 / 10 / 98\end{array}$ & $\begin{array}{l}\text { ARSENIC } \\
\text { CHROMIUM } \\
\text { GROSS ALPHA } \\
\text { LEAD } \\
\text { NITRATE-N } \\
\text { THALLIUM } \\
\text { TOTAL SUSPENDED SOLIDS } \\
\text { URANIUM, TOTAL } \\
\text { ARSENIC } \\
\text { CHROMIUM } \\
\text { GROSS ALPHA } \\
\text { LEAO } \\
\text { NITRATE-N } \\
\text { RADIUM-226 } \\
\text { RADIUM-228 } \\
\text { THALLIUM } \\
\text { THORIUM-228 } \\
\text { THORIUM-230 } \\
\text { THORIUM-232 } \\
\text { TOTAL SUSPENDED SOLIDS } \\
\text { URANIUM, TOTAL } \\
\text { GROSS ALPHA } \\
\text { NITRATE-N } \\
\text { TOTAL SUSPENDED SOLIDS } \\
\text { URANIUM, TOTAL } \\
\text { ARSENIC } \\
\text { CHROMIUM } \\
\text { LEAD } \\
\text { NITRATE-N } \\
\text { THALLIUM } \\
\text { TOTAL SUSPENDED SOLIDS } \\
\text { ARSENIC } \\
\text { CHROMIUM } \\
\text { GROSS ALPHA } \\
\text { LEAD } \\
\text { NITRATE-N } \\
\text { THALLIUM } \\
\text { TOTAL SUSPENDED SOLIDS } \\
\text { URANIUM, TOTAL } \\
\text { ARSENIC } \\
\text { CHROMIUM } \\
\text { GROSS ALPHA } \\
\text { LEAD } \\
\text { NITRATE-N } \\
\text { RADIUM-226 } \\
\text { RADIUM-228 } \\
\text { THALLIUM } \\
\text { THORIUM-228 } \\
\text { THORIUM-230 } \\
\text { THORIUM-232 } \\
\text { TOTAL SUSPENDED SOLIDS } \\
\text { URANIUM, TOTAL } \\
\text { ARSENIC } \\
\text { CHRONIUM } \\
\text { GROSS ALPHA } \\
\text { LEAD } \\
\text { NITRATE-N } \\
\text { RADIUM-226 } \\
\text { RAM }\end{array}$ & $\begin{array}{l}4.5 \\
21.9 \\
12.2 \\
5.7 \\
0.989 \\
\text { ND } \\
72.0 \\
17.4 \\
\text { ND } \\
2.4 \\
1.75 \\
\text { ND } \\
0.79 \\
0.294 \\
0.426 \\
\text { ND } \\
C 0.07 \\
10.07 \\
C 0.05 \\
29.0 \\
21.0 \\
54.5 \\
1.85 \\
718 \\
63.0 \\
7.6 \\
14.8 \\
4.9 \\
0.71 \\
4.9 \\
203 \\
\text { ND } \\
5.7 \\
6.77 \\
2.2 \\
0.860 \\
\text { ND } \\
73.0 \\
8.75 \\
2.2 \\
6.4 \\
47.8 \\
1.6 \\
6.66 \\
0.270 \\
10.32 \\
\text { MD } \\
0.244 \\
0.732 \\
0.272 \\
42.0 \\
52.0 \\
\text { ND } \\
1.6 \\
96.0 \\
\text { ND } \\
5.90 \\
0.103\end{array}$ & $\begin{array}{l}1.3 \\
0.60 \\
1.06 \\
0.90 \\
0.050 \\
1.8 \\
1.00 \\
0.677 \\
1.3 \\
0.60 \\
0.624 \\
0.90 \\
0.050 \\
0.027 \\
0.361 \\
1.8 \\
0.103 \\
0.079 \\
0.054 \\
1.00 \\
0.677 \\
0.909 \\
0.10 \\
1.00 \\
0.677 \\
2.4 \\
0.60 \\
2.9 \\
0.02 \\
4.8 \\
10.0 \\
2.1 \\
0.80 \\
0.766 \\
1.1 \\
0.050 \\
2.9 \\
1.00 \\
0.677 \\
1.3 \\
0.60 \\
0.896 \\
0.90 \\
1.00 \\
0.035 \\
0.363 \\
1.8 \\
0.062 \\
0.044 \\
0.013 \\
1.00 \\
0.677 \\
1.3 \\
0.60 \\
0.624 \\
0.90 \\
0.50 \\
0.029\end{array}$ & $\begin{array}{l}\text { UG/L } \\
\text { UG } / L \\
P C I / L \\
U G / L \\
M G / L \\
\text { UG/L } \\
M G / L \\
P C I / L \\
\text { UG/L } \\
\text { UG/L } \\
P C I / L \\
U G / L \\
M G / L \\
P C I / L \\
P C I / L \\
U G / L \\
P C I / L \\
P C I / L \\
P C I / L \\
M G / L \\
P C I / L \\
P C I / L \\
M G / L \\
M G / L \\
P C I / L \\
U G / L \\
U G / L \\
U G / L \\
M G / L \\
U G / L \\
M G / L \\
U G / L \\
U G / L \\
P C I / L \\
U G / L \\
M G / L \\
U G / L \\
M G / L \\
P C I / L \\
U G / L \\
U G / L \\
P C I / L \\
U G / L \\
M G / L \\
P C I / L \\
P C I / L \\
U G / L \\
P C I / L \\
P C I / L \\
P C I / L \\
M G / L \\
P C I / L \\
U G / L \\
U G / L \\
P C I / L \\
U G / L \\
M G / L \\
P C I / L \\
\end{array}$ & $\begin{array}{l}\text { EPA CLP } \\
\text { EPA CLP } \\
\text { EPA } 900.0 \\
\text { EPA CLP } \\
\text { EPA } 353.1 \\
\text { EPA CLP } \\
\text { EPA } 160.2 \\
\text { ASTM } 5174-91 \\
\text { EPA CLP } \\
\text { EPA CLP } \\
\text { EPA } 900.0 \\
\text { EPA CLP } \\
\text { EPA } 353.1 \\
\text { EPA } 904.0 \\
\text { EPA } 904.0 \\
\text { EPA CLP } \\
\text { NAS-NS-3004 } \\
\text { NAS-NS-3004 } \\
\text { NAS-NS-3004 } \\
\text { EPA } 160.2 \\
\text { ASTM } 5174-91 \\
\text { EPA } 900.0 \\
\text { EPA } 353.1 \\
\text { EPA } 160.2 \\
\text { ASTM } 5174-91 \\
\text { EPA CLP } \\
\text { EPA CLP } \\
\text { EPA CLP } \\
\text { EPA } 353.2 \\
\text { EPA CLP } \\
\text { EPA } 160.2 \\
\text { EPA CLP } \\
\text { EPA CLP } \\
\text { EPA } 900.0 \\
\text { EPA CLP } \\
\text { EPA } 353.1 \\
\text { EPA CLP } \\
\text { EPA } 160.2 \\
\text { ASTM } 5174-91 \\
\text { EPA CLP } \\
\text { EPA CLP } \\
\text { EPA } 900.0 \\
\text { EPA CLP } \\
\text { EPA } 353.1 \\
\text { EPA } 904.0 \\
\text { EPA } 904.0 \\
\text { EPA CLP } \\
\text { NAS-NS-3004 } \\
\text { NAS-NS-3004 } \\
\text { NAS-NS-3004 } \\
\text { EPA } 160.2 \\
\text { ASTM } 5174-91 \\
\text { EPA CLP } \\
\text { EPA CLP } \\
\text { EPA } 900.0 \\
\text { EPA CLP. } \\
\text { EPA } 353.1 \\
\text { EPA } 904.0 \\
\end{array}$ \\
\hline
\end{tabular}




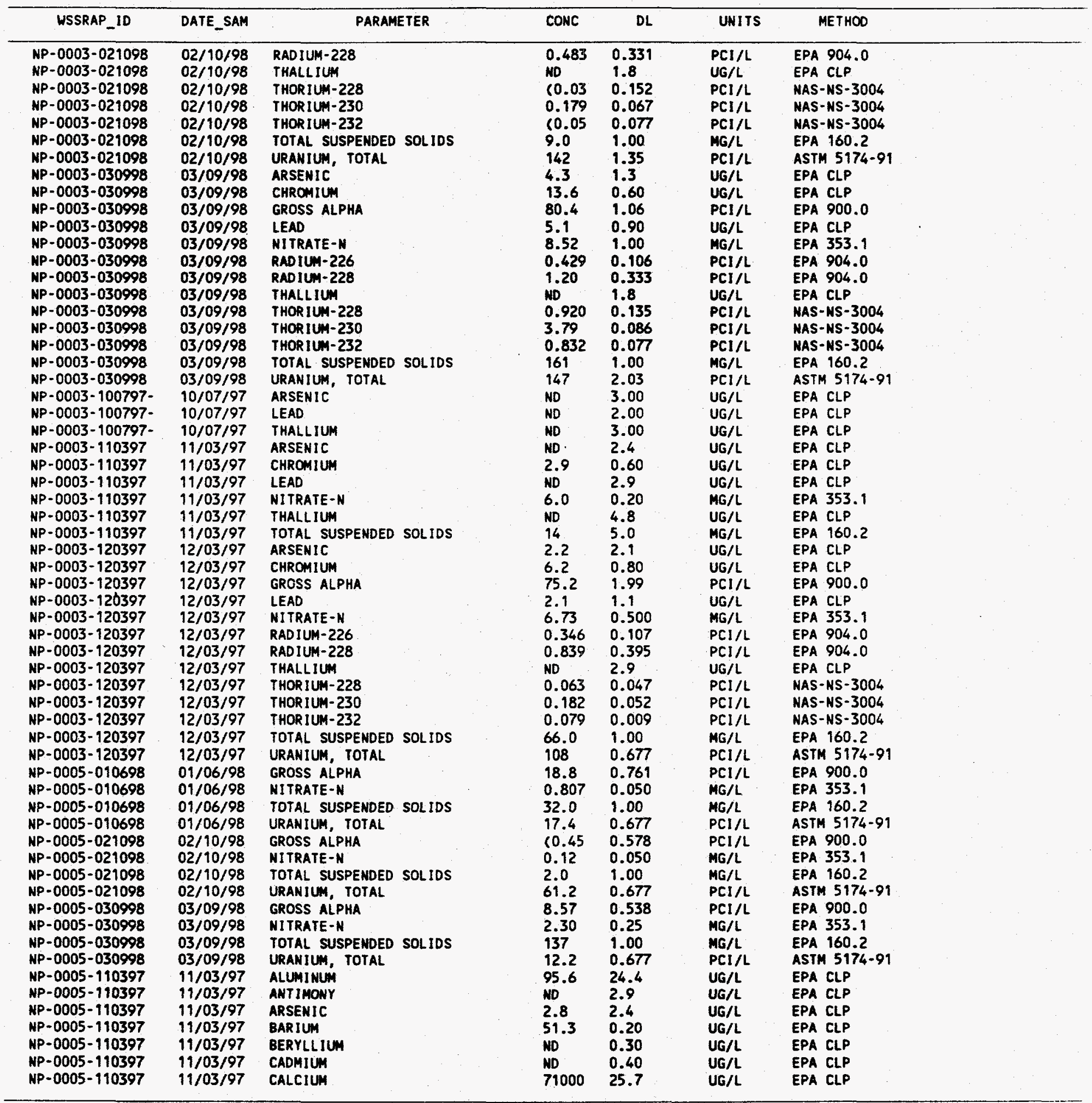




\begin{tabular}{|c|c|c|c|c|c|c|}
\hline WSSI & DATE_SAM & PARAMETER & $\begin{array}{l}\text { CONC } \\
0.97 \\
N D \\
4.9 \\
180\end{array}$ & $\mathrm{DL}$ & UNITS & METHOD \\
\hline 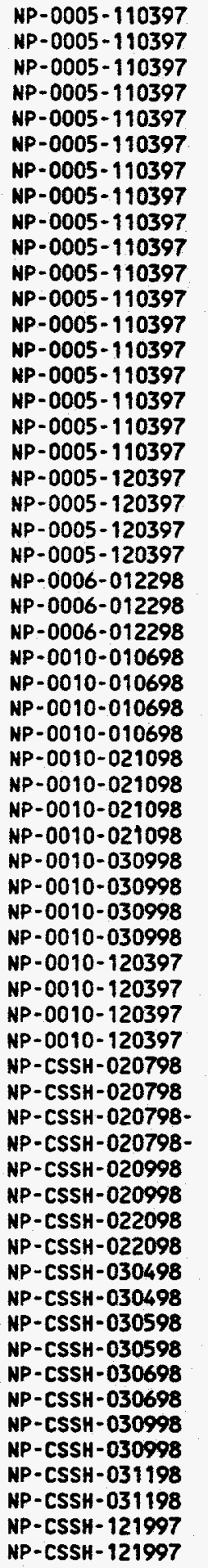 & $\begin{array}{l}11 / 03 / 97 \\
11 / 03 / 97 \\
11 / 03 / 97 \\
11 / 03 / 97 \\
11 / 03 / 97 \\
11 / 03 / 97 \\
11 / 03 / 97 \\
11 / 03 / 97 \\
11 / 03 / 97 \\
11 / 03 / 97 \\
11 / 03 / 97 \\
11 / 03 / 97 \\
11 / 03 / 97 \\
11 / 03 / 97 \\
111 / 03 / 97 \\
11 / 03 / 97 \\
11 / 03 / 97 \\
11 / 03 / 97 \\
12 / 03 / 97 \\
12 / 03 / 97 \\
12 / 03 / 97 \\
12 / 03 / 97 \\
01 / 22 / 98 \\
01 / 22 / 98 \\
01 / 22 / 98 \\
01 / 06 / 98 \\
01 / 06 / 98 \\
01 / 06 / 98 \\
01 / 06 / 98 \\
02 / 10 / 98 \\
02 / 10 / 98 \\
02 / 10 / 98 \\
02 / 10 / 98 \\
03 / 09 / 98 \\
03 / 09 / 98 \\
03 / 09 / 98 \\
03 / 09 / 98 \\
12 / 03 / 97 \\
12 / 03 / 97 \\
12 / 03 / 97 \\
12 / 03 / 97 \\
02 / 07 / 98 \\
02 / 07 / 98 \\
02 / 07 / 98 \\
02 / 07 / 98 \\
02 / 09 / 98 \\
02 / 09 / 98 \\
02 / 20 / 98 \\
02 / 20 / 98 \\
03 / 04 / 98 \\
03 / 04 / 98 \\
03 / 05 / 98 \\
03 / 05 / 98 \\
03 / 06 / 98 \\
03 / 06 / 98 \\
03 / 09 / 98 \\
03 / 09 / 98 \\
03 / 11 / 98 \\
03 / 11 / 98 \\
12 / 19 / 97 \\
12 / 19 / 97\end{array}$ & 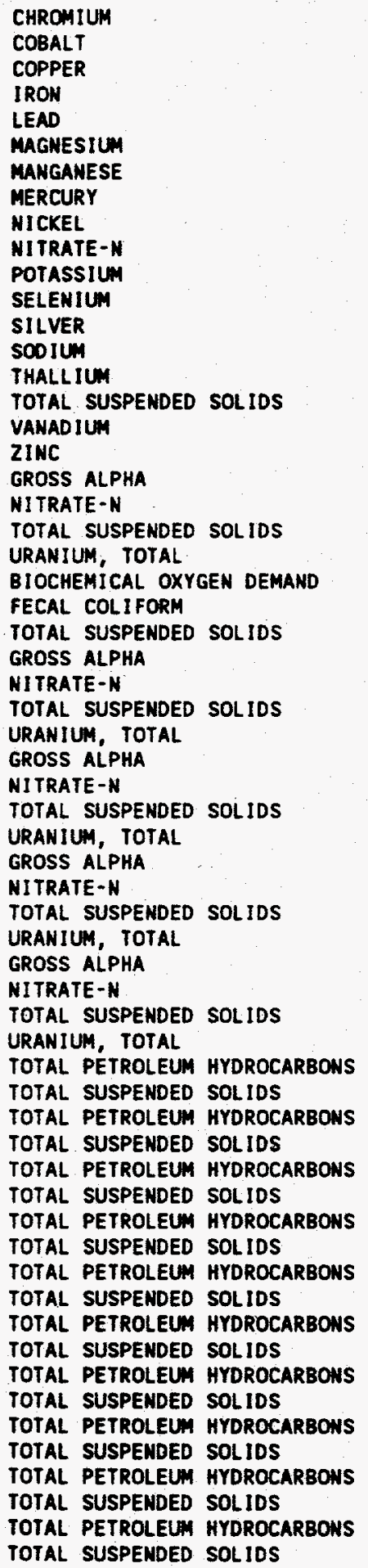 & $\begin{array}{l}0.97 \\
\text { ND } \\
4.9 \\
180 \\
\text { ND } \\
10800 \\
30.9 \\
\text { ND } \\
\text { ND } \\
0.49 \\
3430 \\
\text { ND } \\
\text { ND } \\
20000 \\
\text { ND } \\
\text { ND } \\
1.5 \\
26.1 \\
23.0 \\
0.228 \\
35.0 \\
31.2 \\
\text { ND } \\
\text { ND } \\
9.00 \\
4.50 \\
\text { ND } \\
30.0 \\
2.05 \\
28.2 \\
0.11 \\
2.0 \\
1.58 \\
3.29 \\
0.30 \\
55.0 \\
28.1 \\
2.63 \\
0.054 \\
21.0 \\
1.69 \\
3.21 \\
6.00 \\
1.57 \\
4.00 \\
2.87 \\
140 \\
0.370 \\
6.00 \\
0.370 \\
45.0 \\
\text { ND } \\
42.0 \\
\text { ND } \\
113 \\
\text { ND } \\
36.0 \\
\text { ND } \\
141 \\
\text { ND } \\
7.00 \\
\end{array}$ & 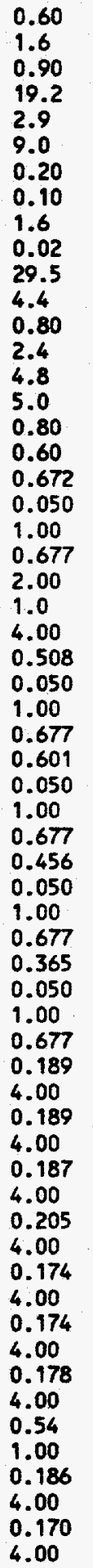 & 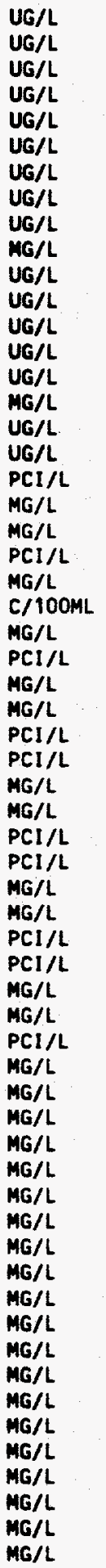 & $\begin{array}{l}\text { EPA CLP } \\
\text { EPA CLP } \\
\text { EPA CLP } \\
\text { EPA CLP } \\
\text { EPA CLP } \\
\text { EPA CLP } \\
\text { EPA CLP } \\
\text { EPA CLP } \\
\text { EPA CLP } \\
\text { EPA } 353.2 \\
\text { EPA } C L P \\
\text { EPA } C L P \\
\text { EPA } C L P \\
\text { EPA } C L P \\
\text { EPA } C L P \\
\text { EPA } 160.2 \\
\text { EPA } C L P \\
\text { EPA CLP } \\
\text { EPA } 900.0 \\
\text { EPA } 353.1 \\
\text { EPA } 160.2 \\
\text { ASTM } 5174-91 \\
\text { EPA } 405.1 \\
\text { SM } 9222 \quad 0 \\
\text { EPA } 160.2 \\
\text { EPA } 900.0 \\
\text { EPA } 353.1 \\
\text { EPA } 160.2 \\
\text { ASTM } 5174-91 \\
\text { EPA } 900.0 \\
\text { EPA } 353.1 \\
\text { EPA } 160.2 \\
\text { ASTM } 5174-91 \\
\text { EPA } 900.0 \\
\text { EPA } 353.1 \\
\text { EPA } 160.2 \\
\text { ASTM } 5174-91 \\
\text { EPA } 900.0 \\
\text { EPA } 353.1 \\
\text { EPA } 160.2 \\
\text { ASTM } 5174-91 \\
\text { EPA } 418.1 \\
\text { EPA } 160.2 \\
\text { EPA } 418.1 \\
\text { EPA } 160.2 \\
\text { EPA } 418.1 \\
\text { EPA } 160.2 \\
\text { EPA } 418.1 \\
\text { EPA } 160.2 \\
\text { EPA } 418.1 \\
\text { EPA } 160.2 \\
\text { EPA } 418.1 \\
\text { EPA } 160.2 \\
\text { EPA } 418.1 \\
\text { EPA } 160.2 \\
\text { EPA } 418.1 \\
\text { EPA } 160.2 \\
\text { EPA } 418.1 \\
\text { EPA } 160.2 \\
\text { EPA } 418.1 \\
\text { EPA } 160.2\end{array}$ \\
\hline
\end{tabular}




\begin{tabular}{|c|c|c|c|c|c|c|}
\hline WSSRAP_ID & DATE_SAM & PARAMETER & CONC & $D L$ & UNITS & METHOO \\
\hline 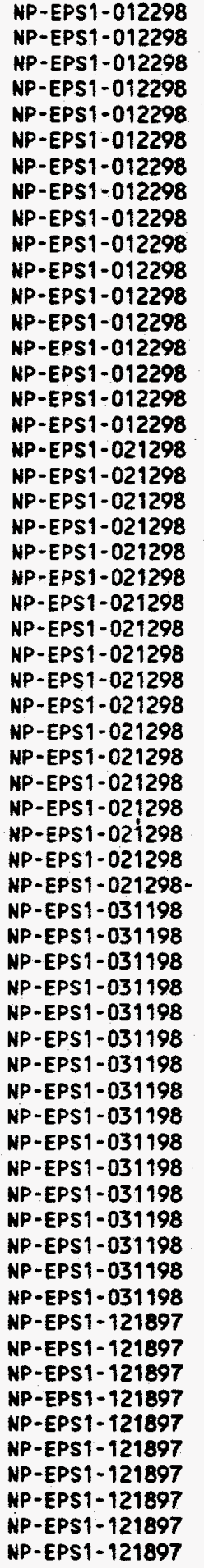 & $\begin{array}{l}01 / 22 / 98 \\
01 / 22 / 98 \\
01 / 22 / 98 \\
01 / 22 / 98 \\
01 / 22 / 98 \\
01 / 22 / 98 \\
01 / 22 / 98 \\
01 / 22 / 98 \\
01 / 22 / 98 \\
01 / 22 / 98 \\
01 / 22 / 98 \\
01 / 22 / 98 \\
01 / 22 / 98 \\
01 / 22 / 98 \\
01 / 22 / 98 \\
01 / 22 / 98 \\
01 / 22 / 98 \\
02 / 12 / 98 \\
02 / 12 / 98 \\
02 / 12 / 98 \\
02 / 12 / 98 \\
02 / 12 / 98 \\
02 / 12 / 98 \\
02 / 12 / 98 \\
02 / 12 / 98 \\
02 / 12 / 98 \\
02 / 12 / 98 \\
02 / 12 / 98 \\
02 / 12 / 98 \\
02 / 12 / 98 \\
02 / 12 / 98 \\
02 / 12 / 98 \\
02 / 12 / 98 \\
02 / 12 / 98 \\
02 / 12 / 98 \\
03 / 11 / 98 \\
03 / 11 / 98 \\
03 / 11 / 98 \\
03 / 11 / 98 \\
03 / 11 / 98 \\
03 / 11 / 98 \\
03 / 11 / 98 \\
03 / 11 / 98 \\
03 / 11 / 98 \\
03 / 11 / 98 \\
03 / 11 / 98 \\
03 / 11 / 98 \\
03 / 11 / 98 \\
03 / 11 / 98 \\
03 / 11 / 98 \\
03 / 11 / 98 \\
12 / 18 / 97 \\
12 / 18 / 97 \\
12 / 18 / 97 \\
12 / 18 / 97 \\
12 / 18 / 97 \\
12 / 18 / 97 \\
12 / 18 / 97 \\
12 / 18 / 97 \\
12 / 18 / 97 \\
12 / 18 / 97\end{array}$ & 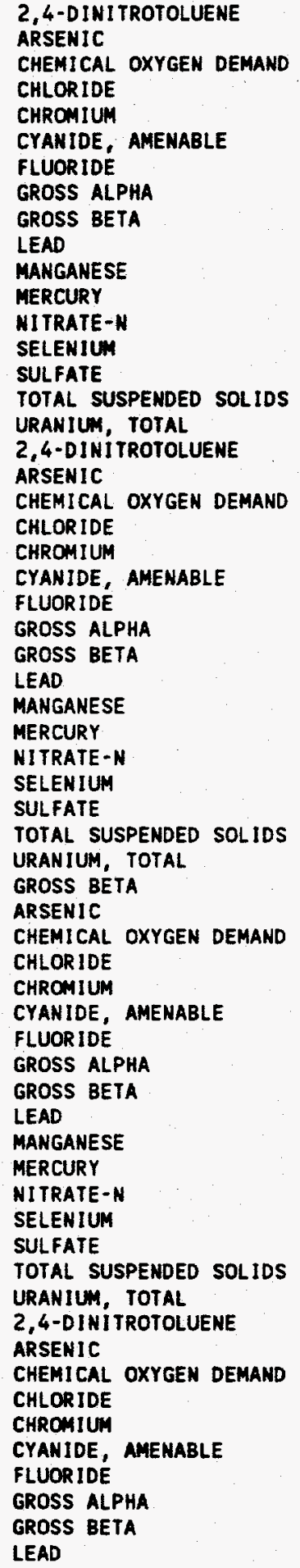 & $\begin{array}{l}\text { ND } \\
3.8 \\
20.0 \\
126 \\
1.7 \\
43.3 \\
1.47 \\
4.36 \\
10.4 \\
\text { ND } \\
3.2 \\
\text { ND } \\
5.16 \\
3.6 \\
459 \\
3.00 \\
1.41 \\
\text { ND } \\
2.40 \\
18.1 \\
150 \\
2.40 \\
\text { ND } \\
(1.10 \\
(3.67 \\
70.4 \\
\text { ND } \\
2.70 \\
\text { ND } \\
60 \\
16.3 \\
590 \\
22 \\
0.733 \\
14.8 \\
\text { ND } \\
10.0 \\
50.7 \\
3.2 \\
\text { ND } \\
1.54 \\
1.38 \\
12.8 \\
\text { ND } \\
2.6 \\
\text { ND } \\
33.3 \\
10.4 \\
324 \\
3.0 \\
\text { ND } \\
\text { ND } \\
\text { ND } \\
\text { ND } \\
103 \\
0.83 \\
\text { ND } \\
2.1 \\
6.16 \\
12.8 \\
\text { ND }\end{array}$ & $\begin{array}{l}0.03 \\
1.3 \\
5.00 \\
8.00 \\
0.00 \\
5.00 \\
0.50 \\
1.04 \\
1.13 \\
0.90 \\
0.30 \\
0.10 \\
1.00 \\
2.2 \\
12.5 \\
1.00 \\
0.67 \\
0.20 \\
2.1 \\
10.0 \\
20 \\
1.0 \\
5.0 \\
2.0 \\
0\end{array}$ & $\begin{array}{l}U G / L \\
U G / L \\
M G / L \\
M G / L \\
U G / L \\
U G / L \\
M G / L \\
P C I / L \\
P C I / L \\
U G / L \\
U G / L \\
U G / L \\
M G / L \\
U G / L \\
M G / L \\
M G / L \\
P C I / L \\
U G / L \\
U G / L \\
M G / L \\
M G / L \\
U G / L \\
U G / L \\
M G / L \\
P C I / L \\
P C I / L \\
U G / L \\
U G / L \\
U G / L \\
M G / L \\
U G / L \\
M G / L \\
M G / L \\
P C I / L \\
P C I / L \\
U G / L \\
M G / L \\
M G / L \\
U G / L \\
U G / L \\
M G / L \\
P C I / L \\
P C I / L \\
U G / L \\
U G / L \\
U G / L \\
M G / L \\
U G / L \\
M G / L \\
M G / L \\
P C I / L \\
U G / L \\
U G / L \\
M G / L \\
M G / L \\
U G / L \\
U G / L \\
M G / L \\
P C I / L \\
P C I / L \\
U G / L \\
\end{array}$ & $\begin{array}{l}\text { USATHAMA } \\
\text { EPA } 200.7 \\
\text { EPA } 410.4 \\
\text { EPA } 300.0 \\
\text { EPA } 200.7 \\
\text { EPA } 9010 \\
\text { EPA } 300.0 \\
\text { EPA } 900.0 \\
\text { EPA } 900.0 \\
\text { EPA } 200.7 \\
\text { EPA } 200.7 \\
\text { EPA } 245.1 \\
\text { EPA } 353.1 \\
\text { EPA } 200.7 \\
\text { EPA } 300.0 \\
\text { EPA } 160.2 \\
\text { ASTM } 5174-91 \\
\text { SW846 } 8330 \\
\text { EPA } 200 \\
\text { EPA } 410.0 \\
\text { EPA } 300.0 \\
\text { EPA } 200 \\
\text { EPA } 335.1 \\
\text { EPA } 300.0 \\
\text { EPA } 900.0 \\
\text { EPA } 900.0 \\
\text { EPA } 200 \\
\text { EPA } 200 \\
\text { EPA } 200 \\
\text { EPA } 300.0 \\
\text { EPA } 200 \\
\text { EPA } 300.0 \\
\text { EPA } 160.2 \\
\text { ASTM } 5174-91 \\
\text { EPA } 900.0 \\
\text { EPA } 200.7 \\
\text { EPA } 410.4 \\
\text { EPA } 300.0 \\
\text { EPA } 200.7 \\
\text { EPA } 9010 \\
\text { EPA } 300.0 \\
\text { EPA } 900.0 \\
\text { EPA } 900.0 \\
\text { EPA } 200.7 \\
\text { EPA } 200.7 \\
\text { EPA } 245.1 \\
\text { EPA } 353.1 \\
\text { EPA } 200.7 \\
\text { EPA } 300.0 \\
\text { EPA } 160.2 \\
\text { ASTM } 5174-91 \\
\text { SWB46 } 8330 \\
\text { EPA } 200.7 \\
\text { EPA } 410.4 \\
\text { EPA } 300.0 \\
\text { EPA } 200.7 \\
\text { EPA } 335.2 \\
\text { EPA } 300.0 \\
\text { EPA } 900.0 \\
\text { EPA } 900.0 \\
\text { EPA } 200.7 \\
\end{array}$ \\
\hline
\end{tabular}




\begin{tabular}{|c|c|c|c|c|c|c|}
\hline WSSRAP_ID & DATE_SAM & PARAMETER & conc & DL & UN1TS & METHOD \\
\hline 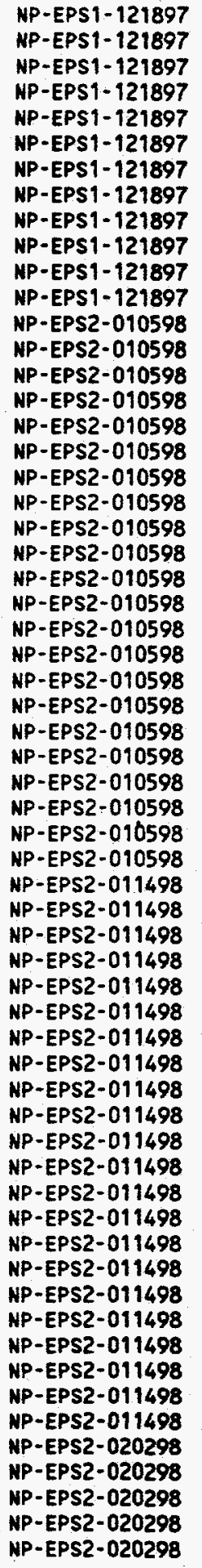 & $\begin{array}{l}12 / 18 / 97 \\
12 / 18 / 97 \\
12 / 18 / 97 \\
12 / 18 / 97 \\
12 / 18 / 97 \\
12 / 18 / 97 \\
12 / 18 / 97 \\
12 / 18 / 97 \\
12 / 18 / 97 \\
12 / 18 / 97 \\
12 / 18 / 97 \\
12 / 18 / 97 \\
01 / 05 / 98 \\
01 / 05 / 98 \\
01 / 05 / 98 \\
01 / 05 / 98 \\
01 / 05 / 98 \\
01 / 05 / 98 \\
01 / 05 / 98 \\
01 / 05 / 98 \\
01 / 05 / 98 \\
01 / 05 / 98 \\
01 / 05 / 98 \\
01 / 05 / 98 \\
01 / 05 / 98 \\
01 / 05 / 98 \\
01 / 05 / 98 \\
01 / 05 / 98 \\
01 / 05 / 98 \\
01 / 05 / 98 \\
01 / 05 / 98 \\
01 / 05 / 98 \\
01 / 05 / 98 \\
01105 / 98 \\
011 / 14 / 98 \\
01 / 14 / 98 \\
01 / 14 / 98 \\
01 / 14 / 98 \\
01 / 14 / 98 \\
011 / 14 / 98 \\
01 / 14 / 98 \\
01 / 14 / 98 \\
01 / 14 / 98 \\
011 / 14 / 98 \\
01 / 14 / 98 \\
01 / 14 / 98 \\
01 / 14 / 98 \\
01 / 14 / 98 \\
011 / 14 / 98 \\
01 / 14 / 98 \\
01 / 14 / 98 \\
01 / 14 / 98 \\
01 / 14 / 98 \\
011 / 14 / 98 \\
01 / 14 / 98 \\
01 / 14 / 98 \\
02 / 02 / 98 \\
02 / 02 / 98 \\
02 / 02 / 98 \\
02 / 02 / 98 \\
02 / 02 / 98\end{array}$ & 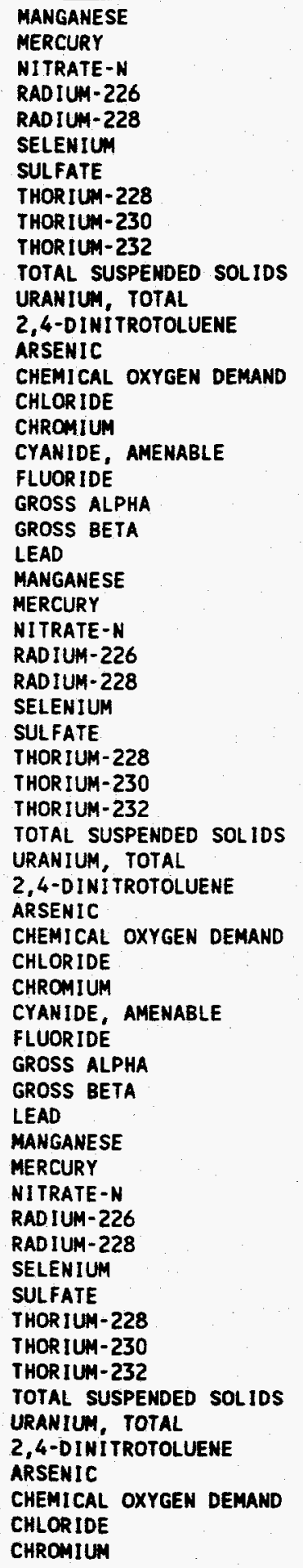 & $\begin{array}{l}5.5 \\
\text { ND } \\
13.6 \\
0.659 \\
4.44 \\
3.8 \\
290 \\
60.35 \\
60.26 \\
0.19 \\
\text { ND } \\
2.79 \\
\text { ND } \\
10.5 \\
\text { ND } \\
123 \\
2.5 \\
6.7 \\
1.10 \\
3.68 \\
6.60 \\
\text { ND } \\
50.4 \\
\text { ND } \\
8.93 \\
0.520 \\
1.06 \\
4.7 \\
383 \\
\text { ND } \\
0.241 \\
10.02 \\
29 \\
2.83 \\
\text { ND } \\
5.3 \\
20.0 \\
112 \\
0.77 \\
\text { ND } \\
1.49 \\
3.73 \\
10.2 \\
\text { ND } \\
3.5 \\
\text { ND } \\
8.76 \\
0.460 \\
0.943 \\
6.7 \\
377 \\
10.04 \\
0.114 \\
10.00 \\
4.00 \\
3.49 \\
\text { ND } \\
5.20 \\
\text { ND } \\
228 \\
4.30 \\
\end{array}$ & $\begin{array}{l}0.20 \\
0.10 \\
1.0 \\
0.488 \\
2.40 \\
0.55 \\
25.0 \\
0.518 \\
0.324 \\
0.324 \\
5.0 \\
0.677 \\
0.015 \\
3.0 \\
5.0 \\
12.5 \\
1.0 \\
5.0 \\
0.05 \\
0.879 \\
0.926 \\
3.0 \\
1.0 \\
0.10 \\
0.40 \\
0.033 \\
0.238 \\
3.0 \\
25.0 \\
0.108 \\
0.037 \\
0.097 \\
2 \\
0.677 \\
0.03 \\
1.3 \\
5.00 \\
8.00 \\
0.60 \\
5.0 \\
0.50 \\
1.06 \\
0.778 \\
0.90 \\
0.30 \\
0.10 \\
1.00 \\
0.120 \\
0.235 \\
2.2 \\
20.0 \\
0.045 \\
0.037 \\
0.025 \\
1.00 \\
0.677 \\
0.04 \\
2.1 \\
10 \\
2 \\
1.0 \\
\\
\\
\end{array}$ & 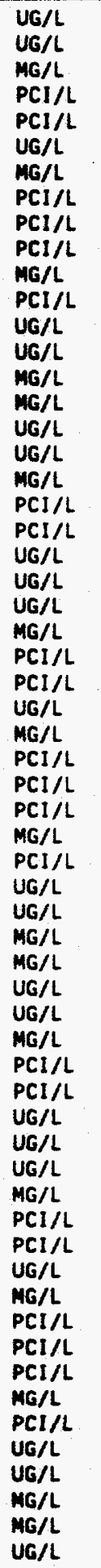 & $\begin{array}{l}\text { EPA } 200.7 \\
\text { EPA } 245.1 \\
\text { EPA } 353.2 \\
\text { EPA } 903.0 \\
\text { EPA } 904.0 \\
\text { EPA } 270.2 \\
\text { EPA } 300.0 \\
\text { EML TH-01 } \\
\text { EML TH-01 } \\
\text { EML TH-01 } \\
\text { EPA } 160.2 \\
\text { ASTM } 5174-91 \\
\text { EPA } 609 \\
\text { EPA } 200.7 \\
\text { EPA } 410.0 \\
\text { EPA } 300.0 \\
\text { EPA } 200.7 \\
\text { EPA } 335.1 \\
\text { EPA } 340.2 \\
\text { EPA } 900.0 \\
\text { EPA } 900.0 \\
\text { EPA } 200.7 \\
\text { EPA } 200.7 \\
\text { EPA } 245.1 \\
\text { EPA } 353.1 \\
\text { EPA } 904.0 \\
\text { EPA } 904.0 \\
\text { EPA } 200.9 \\
\text { EPA } 300.0 \\
\text { NAS-NS-3004 } \\
\text { NAS-NS-3004 } \\
\text { NAS-NS-3004 } \\
\text { EPA } 160.2 \\
\text { ASTM } 5174-91 \\
\text { USATHAMA } \\
\text { EPA } 200.7 \\
\text { EPA } 410.4 \\
\text { EPA } 300.0 \\
\text { EPA } 200.7 \\
\text { EPA } 335.1 \\
\text { EPA } 300.0 \\
\text { EPA } 900.0 \\
\text { EPA } 900.0 \\
\text { EPA } 200.7 \\
\text { EPA } 200.7 \\
\text { EPA } 245.1 \\
\text { EPA } 353.1 \\
\text { EPA } 904.0 \\
\text { EPA } 904.0 \\
\text { EPA } 200.7 \\
\text { EPA } 300.0 \\
\text { NAS-NS-3004 } \\
\text { NAS-NS-3004 } \\
\text { NAS-NS-3004 } \\
\text { EPA } 160.2 \\
\text { ASTM } 5174-91 \\
\text { EPA } 8330 \\
\text { EPA } 200 \\
\text { EPA } 410.0 \\
\text { EPA } 300.0 \\
\text { EPA } 200 \\
\end{array}$ \\
\hline
\end{tabular}




\begin{tabular}{|c|c|c|c|c|c|c|}
\hline WSSRAP_ID & OATE_SAM & PARAMETER & CONC & $D L$ & UNITS & METHOD \\
\hline 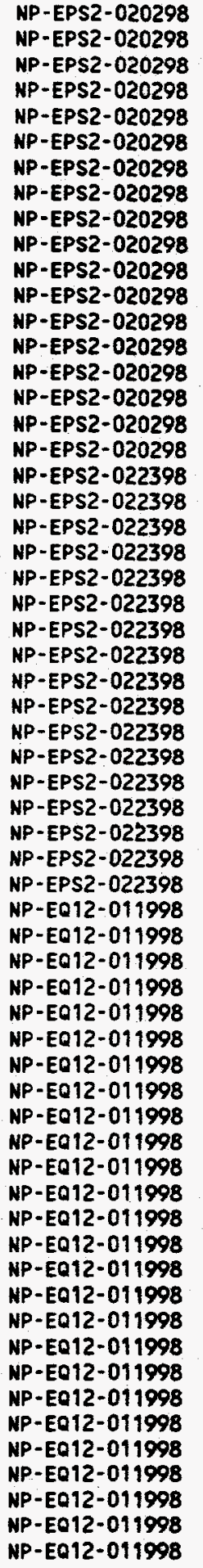 & $\begin{array}{l}02 / 02 / 98 \\
02 / 02 / 98 \\
02 / 02 / 98 \\
02 / 02 / 98 \\
02 / 02 / 98 \\
02 / 02 / 98 \\
02 / 02 / 98 \\
02 / 02 / 98 \\
02 / 02 / 98 \\
02 / 02 / 98 \\
02 / 02 / 98 \\
02 / 02 / 98 \\
02 / 02 / 98 \\
02 / 02 / 98 \\
02 / 02 / 98 \\
02 / 02 / 98 \\
02 / 02 / 98 \\
02 / 02 / 98 \\
02 / 23 / 98 \\
02 / 23 / 98 \\
02 / 23 / 98 \\
02 / 23 / 98 \\
02 / 23 / 98 \\
02 / 23 / 98 \\
02 / 23 / 98 \\
02 / 23 / 98 \\
02 / 23 / 98 \\
02 / 23 / 98 \\
02 / 23 / 98 \\
02 / 23 / 98 \\
02 / 23 / 98 \\
02 / 23 / 98 \\
02 / 23 / 98 \\
02 / 23 / 98 \\
02 / 23 / 98 \\
01 / 19 / 98 \\
01 / 19 / 98 \\
01 / 19 / 98 \\
01 / 19 / 98 \\
01 / 19 / 98 \\
01 / 19 / 98 \\
01 / 19 / 98 \\
01 / 19 / 98 \\
01 / 19 / 98 \\
01 / 19 / 98 \\
01 / 19 / 98 \\
01 / 19 / 98 \\
01 / 19 / 98 \\
01 / 19 / 98 \\
01 / 19 / 98 \\
01 / 19 / 98 \\
01 / 19 / 98 \\
01 / 19 / 98 \\
01 / 19 / 98 \\
01 / 19 / 98 \\
01 / 19 / 98 \\
011 / 19 / 98 \\
01 / 19 / 98 \\
01 / 19 / 98 \\
01 / 19 / 98 \\
01 / 19 / 98\end{array}$ & 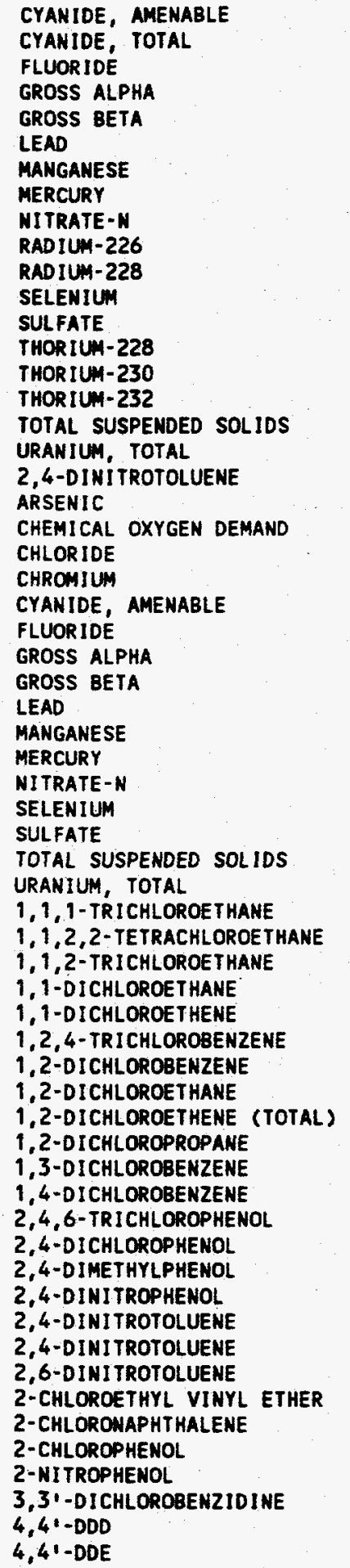 & $\begin{array}{l}\text { ND } \\
\text { ND } \\
1.40 \\
4.85 \\
14.1 \\
0.940 \\
5.20 \\
0.110 \\
34.8 \\
1.09 \\
1.00 \\
\text { ND } \\
510 \\
0.217 \\
0.131 \\
\text { C0.03 } \\
\text { ND } \\
0.813 \\
\text { ND } \\
\text { ND } \\
14.0 \\
76.2 \\
2.7 \\
\text { ND } \\
1.80 \\
5.70 \\
15.9 \\
\text { ND } \\
\text { O.90 } \\
\text { ND } \\
57.7 \\
19.3 \\
444 \\
3.0 \\
\text { ND } \\
\text { ND } \\
\text { ND } \\
\text { ND } \\
\text { ND } \\
\text { ND } \\
\text { ND } \\
\text { ND } \\
\text { ND } \\
\text { ND } \\
\text { ND } \\
\text { ND } \\
\text { ND } \\
\text { ND } \\
\text { ND } \\
\text { ND } \\
\text { ND } \\
\text { ND } \\
\text { ND } \\
\text { ND } \\
\text { ND } \\
\text { ND } \\
\text { ND } \\
\text { ND } \\
\text { ND } \\
\text { ND } \\
\text { ND } \\
\end{array}$ & $\begin{array}{l}5.0 \\
2.0 \\
0.2 \\
1.57 \\
1.68 \\
0.9 \\
0.3 \\
0.1 \\
0.1 \\
0.026 \\
0.276 \\
2.0 \\
2 \\
0.148 \\
0.124 \\
0.148 \\
4 \\
0.677 \\
0.03 \\
1.3 \\
5.00 \\
8.00 \\
0.60 \\
5.00 \\
0.50 \\
1.56 \\
1.59 \\
0.90 \\
0.30 \\
0.10 \\
5.00 \\
2.2 \\
20.0 \\
1.00 \\
0.677 \\
2.50 \\
1.50 \\
2.80 \\
2.50 \\
3.20 \\
1.00 \\
1.00 \\
2.50 \\
2.40 \\
2.00 \\
1.00 \\
1.00 \\
4.50 \\
2.00 \\
2.00 \\
30.0 \\
2.00 \\
0.0270 \\
2.00 \\
3.10 \\
1.00 \\
2.00 \\
2.00 \\
5.00 \\
0.00588 \\
0.00588\end{array}$ & 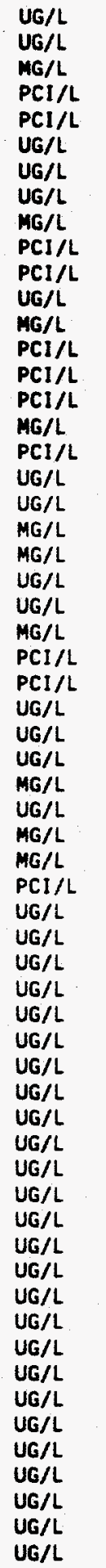 & $\begin{array}{l}\text { EPA } 335.1 \\
\text { EPA } 200 \\
\text { EPA } 300.0 \\
\text { EPA } 900.0 \\
\text { EPA } 900.0 \\
\text { EPA } 200 \\
\text { EPA } 200 \\
\text { EPA } 200 \\
\text { EPA } 300.0 \\
\text { EPA } 904.0 \\
\text { EPA } 904.0 \\
\text { EPA } 200 \\
\text { EPA } 300.0 \\
\text { NAS-NS-3004 } \\
\text { NAS-NS-3004 } \\
\text { NAS-NS-3004 } \\
\text { EPA } 160.2 \\
\text { ASTM } 5174-91 \\
\text { USATHAMA } \\
\text { EPA } 200.7 \\
\text { EPA } 410.4 \\
\text { EPA } 300.0 \\
\text { EPA } 200.7 \\
\text { EPA } 9010 \\
\text { EPA } 300.0 \\
\text { EPA } 900.0 \\
\text { EPA } 900.0 \\
\text { EPA } 200.7 \\
\text { EPA } 200.7 \\
\text { EPA } 245.1 \\
\text { EPA } 353.1 \\
\text { EPA } 200.7 \\
\text { EPA } 300.0 \\
\text { EPA } 160.2 \\
\text { ASTM } 5174-91 \\
\text { EPA } 624 \\
\text { EPA } 624 \\
\text { EPA } 624 \\
\text { EPA } 624 \\
\text { EPA } 624 \\
\text { EPA } 625 \\
\text { EPA } 625 \\
\text { EPA } 624 \\
\text { EPA } 624 \\
\text { EPA } 624 \\
\text { EPA } 625 \\
\text { EPA } 625 \\
\text { EPA } 625 \\
\text { EPA } 625 \\
\text { EPA } 625 \\
\text { EPA } 625 \\
\text { EPA } 625 \\
\text { EPA } 8330 \\
\text { EPA } 625 \\
\text { EPA } 624 \\
\text { EPA } 625 \\
\text { EPA } 625 \\
\text { EPA } 625 \\
\text { EPA } 625 \\
\text { EPA } 608 \\
\text { EPA } 608 \\
\end{array}$ \\
\hline
\end{tabular}




\begin{tabular}{|c|c|c|c|c|c|c|}
\hline WSSRAP_ID & DATE_SAM & PARAMETER & CONC & $D L$ & UNITS & METHOO \\
\hline 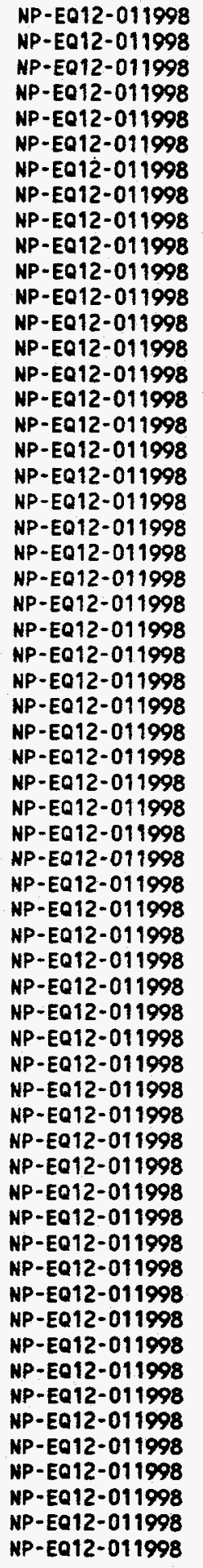 & 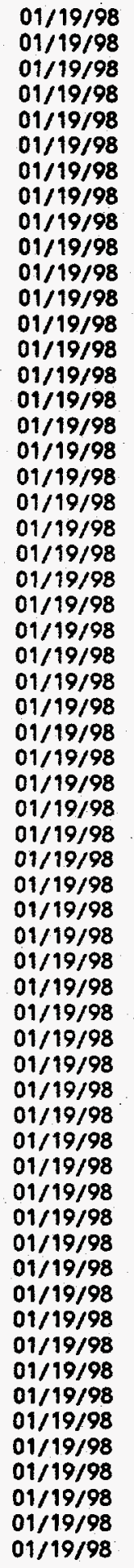 & 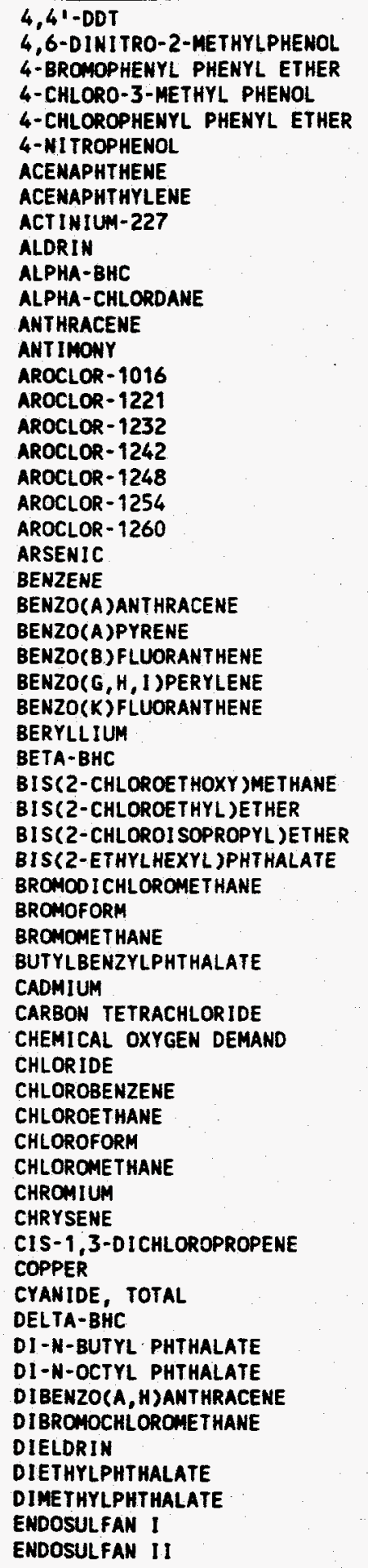 & 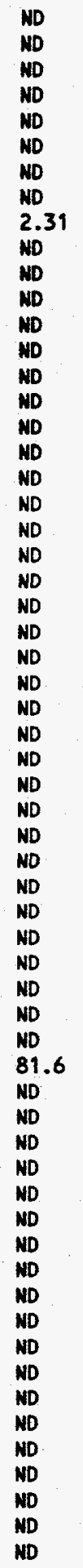 & $\begin{array}{l}0.0235 \\
20.0 \\
1.00 \\
1.50 \\
1.00 \\
10.0 \\
1.00 \\
1.00 \\
16.0 \\
0.00588 \\
0.00588 \\
0.0294 \\
1.00 \\
4.00 \\
0.118 \\
0.118 \\
0.118 \\
0.118 \\
0.118 \\
0.118 \\
0.118 \\
4.00 \\
1.00 \\
1.50 \\
2.00 \\
1.50 \\
2.50 \\
1.50 \\
1.00 \\
0.00588 \\
1.00 \\
1.50 \\
1.00 \\
2.00 \\
2.20 \\
2.60 \\
3.50 \\
1.50 \\
1.00 \\
2.60 \\
5.00 \\
1.00 \\
1.40 \\
8.20 \\
2.50 \\
4.40 \\
2.00 \\
1.50 \\
2.00 \\
2.00 \\
0.00500 \\
0.00588 \\
1.00 \\
2.40 \\
2.50 \\
2.30 \\
0.00588 \\
1.00 \\
2.00 \\
0.00588 \\
0.00588\end{array}$ & $\begin{array}{l}\text { UG/L } \\
U G / L \\
U G / L \\
U G / L \\
U G / L \\
U G / L \\
U G / L \\
U G / L \\
U C I / L \\
U G / L \\
U G / L \\
U G / L \\
U G / L \\
U G / L \\
U G / L \\
U G / L \\
U G / L \\
U G / L \\
U G / L \\
U G / L \\
U G / L \\
U G / L \\
U G / L \\
U G / L \\
U G / \\
U G / L \\
U G / L \\
U G / L \\
U G / L \\
U G / L \\
U G / L \\
U G / L \\
U G / L \\
U G / L \\
U G / L \\
U G / L \\
U G / L \\
U G / L \\
U G / L \\
U G / L \\
U G / L \\
U G / L \\
U G / L \\
U G / L \\
U G / L \\
U G / L \\
U G / L \\
U G / L \\
U G / L \\
U G / L \\
U G / L \\
U G / L \\
U G / L \\
U G / L \\
U G / L \\
U G / L \\
U G / \\
U\end{array}$ & 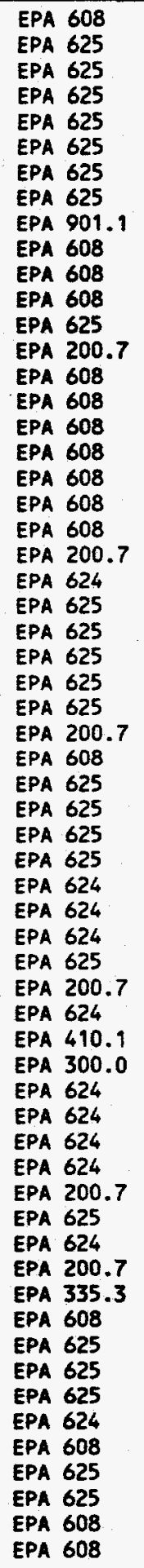 \\
\hline
\end{tabular}




\begin{tabular}{|c|c|c|c|c|c|c|}
\hline WSSRAP_ID & DATE_SAM & PARAMETER & CONC & $\mathrm{OL}$ & UNITS & METHOD \\
\hline 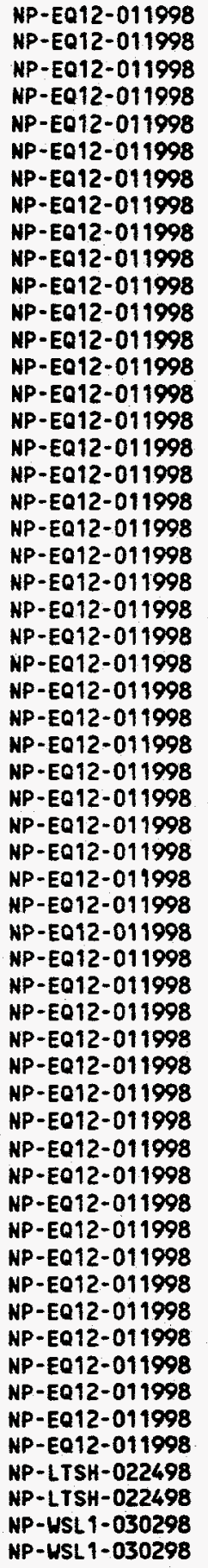 & $\begin{array}{l}01 / 19 / 98 \\
01 / 19 / 98 \\
01 / 19 / 98 \\
01 / 19 / 98 \\
01 / 19 / 98 \\
01 / 19 / 98 \\
01 / 19 / 98 \\
01 / 19 / 98 \\
01 / 19 / 98 \\
01 / 19 / 98 \\
01 / 19 / 98 \\
01 / 19 / 98 \\
01 / 19 / 98 \\
01 / 19 / 98 \\
01 / 19 / 98 \\
01 / 19 / 98 \\
01 / 19 / 98 \\
01 / 19 / 98 \\
01 / 19 / 98 \\
01 / 19 / 98 \\
01 / 19 / 98 \\
01 / 19 / 98 \\
01 / 19 / 98 \\
01 / 19 / 98 \\
01 / 19 / 98 \\
01 / 19 / 98 \\
01 / 19 / 98 \\
01 / 19 / 98 \\
01 / 19 / 98 \\
01 / 19 / 98 \\
01 / 19 / 98 \\
01 / 19 / 98 \\
01 / 19 / 98 \\
01 / 19 / 98 \\
01 / 19 / 98 \\
01 / 19 / 98 \\
01 / 19 / 98 \\
01 / 19 / 98 \\
01 / 19 / 98 \\
01 / 19 / 98 \\
01 / 19 / 98 \\
01 / 19 / 98 \\
01 / 19 / 98 \\
01 / 19 / 98 \\
01 / 19 / 98 \\
01 / 19 / 98 \\
01119 / 98 \\
01 / 19 / 98 \\
01 / 19 / 98 \\
01 / 19 / 98 \\
01 / 19 / 98 \\
01 / 19 / 98 \\
01 / 19 / 98 \\
01 / 19 / 98 \\
02 / 24 / 98 \\
02 / 24 / 98 \\
03 / 02 / 98 \\
03 / 02 / 98\end{array}$ & 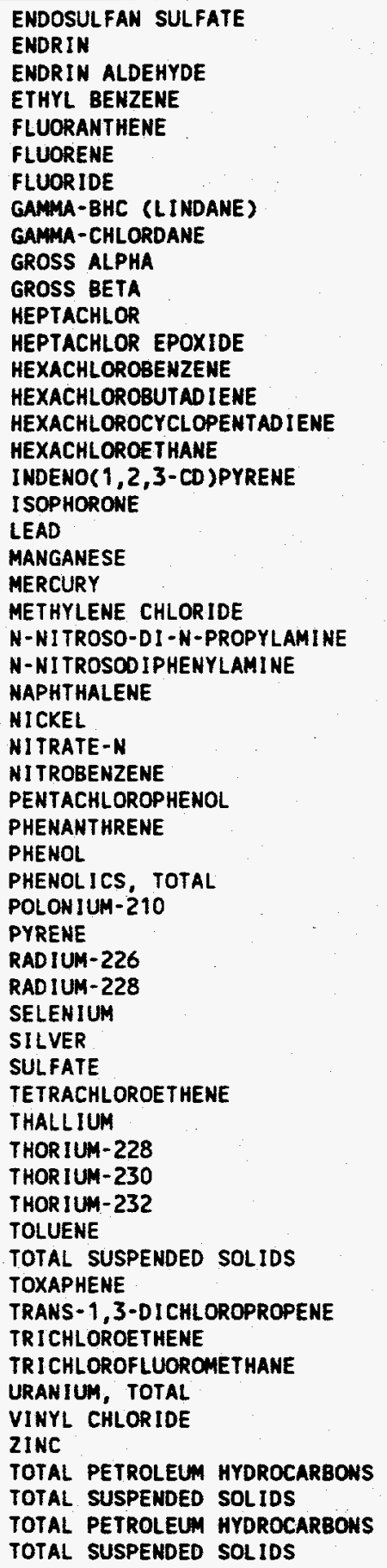 & $\begin{array}{l}\text { ND } \\
\text { ND } \\
\text { ND } \\
\text { ND } \\
\text { ND } \\
\text { ND } \\
\text { ND } \\
\text { ND } \\
\text { ND } \\
0.777 \\
2.27 \\
\text { ND } \\
\text { ND } \\
\text { ND } \\
\text { ND } \\
\text { ND } \\
\text { ND } \\
\text { ND } \\
\text { ND } \\
\text { ND } \\
6.50 \\
\text { ND } \\
C 0.87 \\
\text { ND } \\
\text { ND } \\
\text { ND } \\
\text { ND } \\
0.017 \\
\text { ND } \\
\text { ND } \\
\text { ND } \\
\text { ND } \\
\text { ND } \\
\text { ND } \\
\text { ND } \\
0.150 \\
1.57 \\
\text { ND } \\
\text { ND } \\
86.2 \\
\text { ND } \\
\text { ND } \\
\text { CO.07 } \\
0.458 \\
C 0.05 \\
\text { ND } \\
5.00 \\
\text { ND } \\
\text { ND } \\
\text { ND } \\
\text { ND } \\
\text { ND } \\
\text { ND } \\
\text { 15.5 } \\
0.340 \\
6.00 \\
0.960 \\
\text { ND }\end{array}$ & $\begin{array}{l}0.00588 \\
0.00588 \\
0.00588 \\
1.30 \\
1.00 \\
1.00 \\
0.100 \\
0.00588 \\
0.0294 \\
0.525 \\
0.818 \\
0.00588 \\
0.00588 \\
2.00 \\
2.00 \\
10.0 \\
1.50 \\
2.50 \\
1.00 \\
2.50 \\
1.00 \\
0.100 \\
6.40 \\
1.00 \\
1.00 \\
1.00 \\
3.00 \\
0.0100 \\
1.00 \\
10.0 \\
1.00 \\
2.00 \\
1.76 \\
0.130 \\
1.00 \\
0.097 \\
1.54 \\
4.00 \\
2.00 \\
1.00 \\
1.90 \\
4.00 \\
0.089 \\
0.215 \\
0.0697 \\
1.70 \\
4.00 \\
0.588 \\
1.60 \\
3.00 \\
4.60 \\
0.677 \\
4.60 \\
10.0 \\
0.187 \\
4.00 \\
0.185 \\
4.00\end{array}$ & $\begin{array}{l}\text { UG/L } \\
U G / L \\
U G / L \\
U G / L \\
U G / L \\
U G / L \\
M G / L \\
U G / L \\
U G / L \\
P C I / L \\
P C I / L \\
U G / L \\
U G / L \\
U G / L \\
U G / L \\
U G / L \\
U G / L \\
U G / L \\
U G / L \\
U G / L \\
U G / L \\
U G / L \\
U G / L \\
U G / L \\
U G / L \\
U G / L \\
U G / L \\
M G / L \\
U G / L \\
U G / L \\
U G / L \\
U G / L \\
U G / L \\
P C I / L \\
U G / L \\
P C I / L \\
P C I / L \\
U G / L \\
U G / L \\
M G / L \\
U G / L \\
U G / L \\
P C I / L \\
P C I I / L \\
P C I / L \\
U G / L \\
M G / L \\
U G / L \\
U G / L \\
U G / L \\
U G / L \\
P C I / L \\
U G / L \\
U G / L \\
M G / L \\
M G / L \\
M G / L \\
M G / L\end{array}$ & $\begin{array}{l}\text { EPA } 608 \\
\text { EPA } 608 \\
\text { EPA } 608 \\
\text { EPA } 624 \\
\text { EPA } 625 \\
\text { EPA } 625 \\
\text { EPA } 300.0 \\
\text { EPA } 608 \\
\text { EPA } 608 \\
\text { EPA } 900.0 \\
\text { EPA } 900.0 \\
\text { EPA } 608 \\
\text { EPA } 608 \\
\text { EPA } 625 \\
\text { EPA } 625 \\
\text { EPA } 625 \\
\text { EPA } 625 \\
\text { EPA } 625 \\
\text { EPA } 625 \\
\text { EPA } 200.7 \\
\text { EPA } 200.7 \\
\text { EPA } 61 P \\
\text { EPA } 624 \\
\text { EPA } 625 \\
\text { EPA } 625 \\
\text { EPA } 625 \\
\text { EPA } 200.7 \\
\text { EPA } 353.2 \\
\text { EPA } 625 \\
\text { EPA } 625 \\
\text { EPA } 625 \\
\text { EPA } 625 \\
\text { EPA } 420.2 \\
\text { EML } P 0-02 \\
\text { EPA } 625 \\
\text { EPA } 903.1 \\
\text { EPA } 904.0 \\
\text { EPA } 200.7 \\
\text { EPA } 200.7 \\
\text { EPA } 300.0 \\
\text { EPA } 624 \\
\text { EPA } 200.7 \\
\text { DOERP725 } \\
\text { DOERP725 } \\
\text { DOERP725 } \\
\text { EPA } 624 \\
\text { EPA } 160.2 \\
\text { EPA } 608 \\
\text { EPA } 624 \\
\text { EPA } 624 \\
\text { EPA } 624 \\
\text { ASTM } 5174-91 \\
\text { EPA } 624 \\
\text { EPA } 200.7 \\
\text { EPA } 418.1 \\
\text { EPA } 160.2 \\
\text { EPA } 418.1 \\
\text { EPA } 160.2 \\
\end{array}$ \\
\hline
\end{tabular}




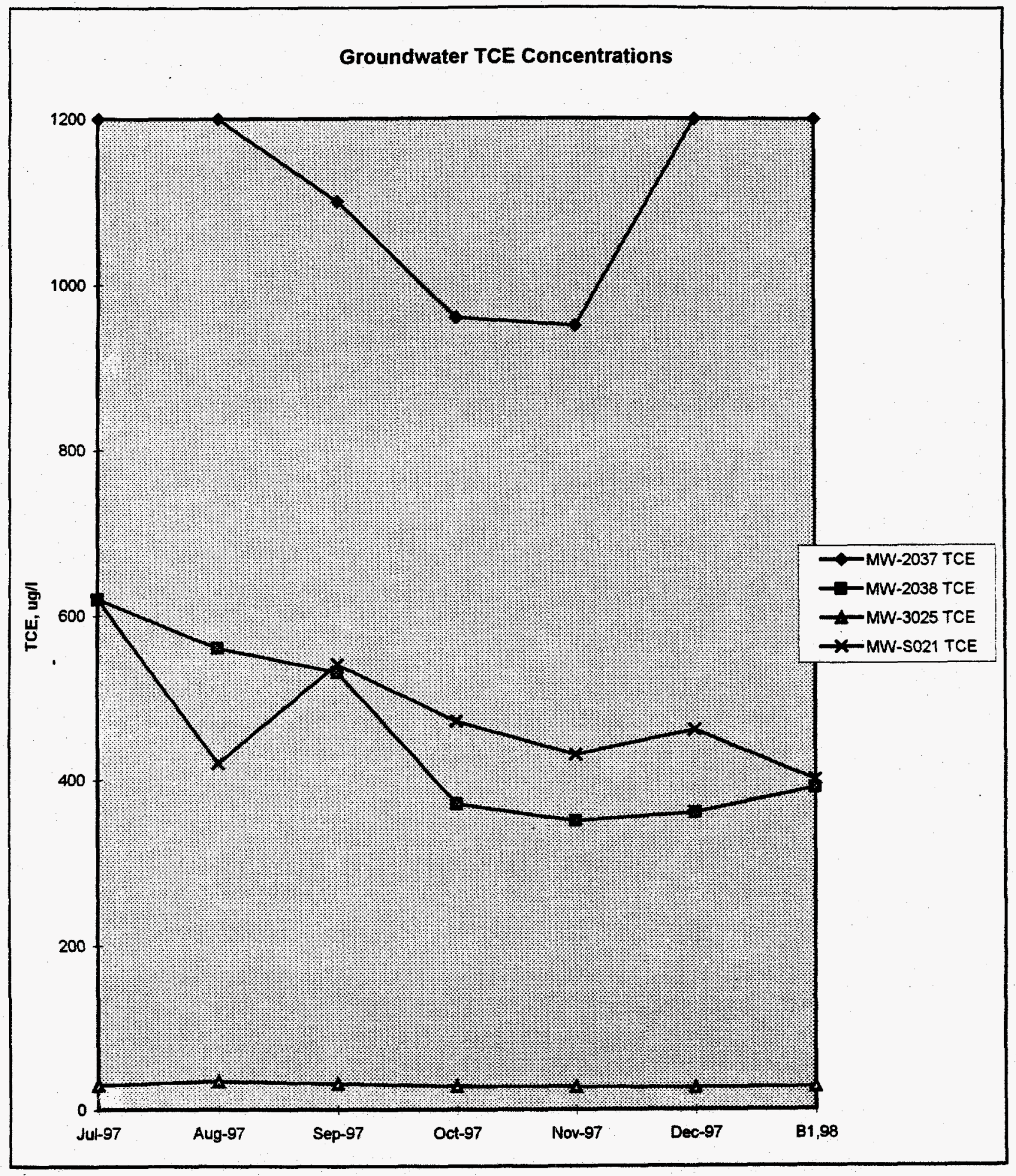




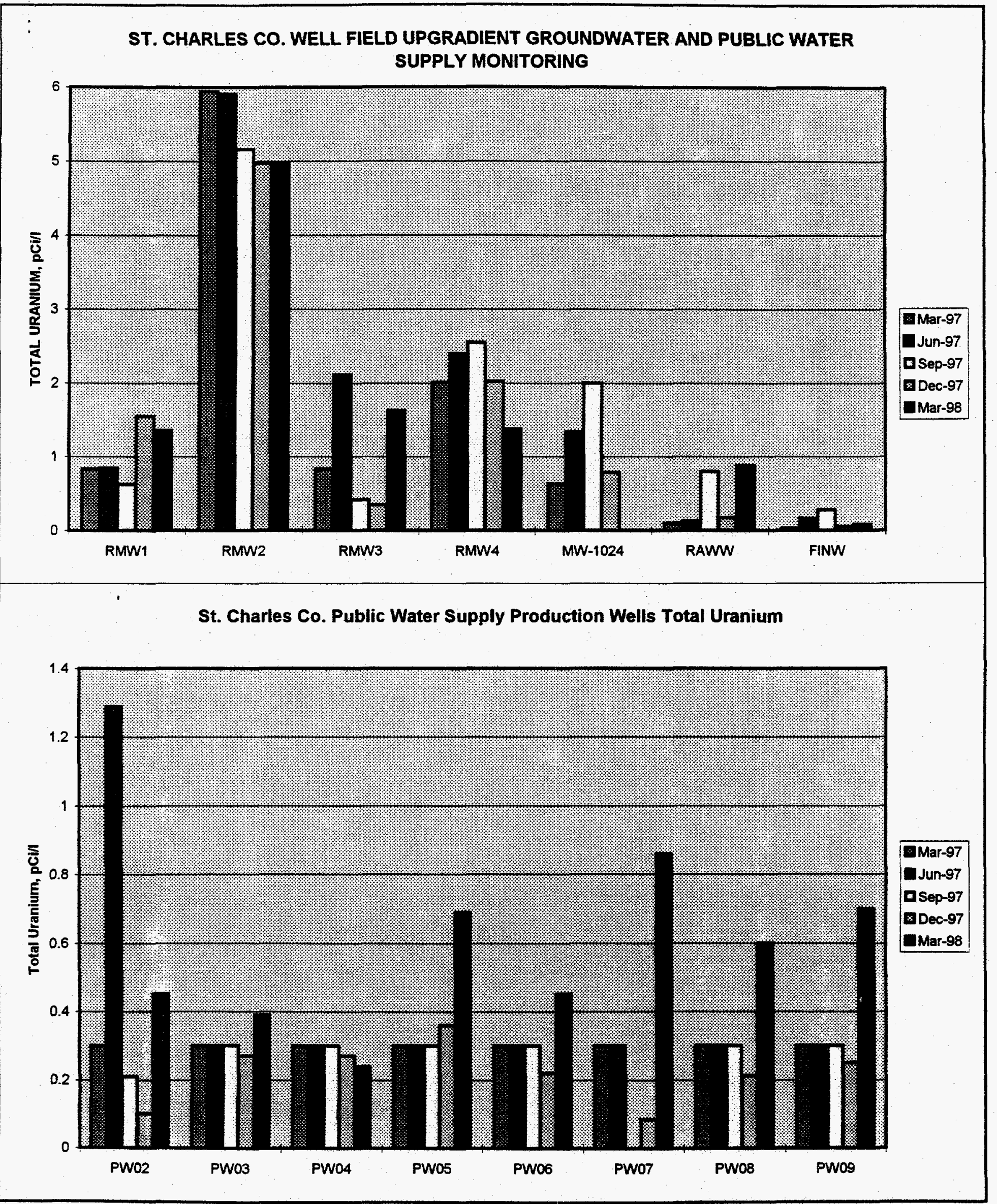




\begin{tabular}{|c|c|c|c|c|c|c|}
\hline WSSRAP_ID & DATE_SAM & PARAMETER & CONC & $\mathrm{DL}$ & UNITS & METHOD \\
\hline $\begin{array}{l}\text { GW-1002-0497 } \\
G W-1002-0497 \\
G W-1002-0497 \\
G W-1002-0497 \\
G W-1002-0497 \\
G W-1002-0497 \\
G W-1002-0497 \\
G W-1002-0497 \\
G W-1002-0198 \\
G W-1002-0198 \\
G W-1002-0198 \\
G W-1002-0198 \\
G W-1002-0198 \\
G W-1002-0198 \\
G W-1002-0198 \\
G W-1004-0497 \\
G W-1004-0497 \\
G W-1004-0497 \\
G H-1004-0497 \\
G W-1004-0497 \\
G W-1004-0497 \\
G W-1004-0497 \\
G W-1004-0198 \\
G W-1004-0198 \\
G W-1004-0198 \\
G W-1004-0198 \\
G W-1004-0198 \\
G W-1004-0198 \\
G W-1004-0198 \\
G W-1005-0497 \\
G W-1005-0497 \\
G W-1005-0497 \\
G W-1005-0497 \\
G W-1005-0497 \\
G W-1005-0497 \\
G W-1005-0497 \\
G W-1006-0497 \\
G W-1006-0497 \\
G W-1006-0497 \\
G W-1006-0497 \\
G W-1006-0497 \\
G W-1006-0497 \\
G W-1006-0497 \\
G W-1006-0198 \\
G W-1006-0198 \\
G W-1006-0198 \\
G W-1006-0198 \\
G W-1006-0198 \\
G W-1006-0198 \\
G W-1006-0198 \\
G W-1007-0497 \\
G W-1007-0497-1007-0497 \\
G-1007-0497 \\
\text { GW }\end{array}$ & $\begin{array}{l}11 / 13 / 97 \\
11 / 13 / 97 \\
11 / 13 / 97 \\
11 / 13 / 97 \\
11 / 13 / 97 \\
11 / 13 / 97 \\
11 / 13 / 97 \\
11 / 13 / 97 \\
02 / 04 / 98 \\
02 / 04 / 98 \\
02 / 04 / 98 \\
02 / 04 / 98 \\
02 / 04 / 98 \\
02 / 04 / 98 \\
02 / 04 / 98 \\
11 / 13 / 97 \\
11 / 13 / 97 \\
11 / 13 / 97 \\
11 / 13 / 97 \\
11 / 13 / 97 \\
11 / 13 / 97 \\
11 / 13 / 97 \\
02 / 04 / 98 \\
02 / 04 / 98 \\
02 / 04 / 98 \\
02 / 04 / 98 \\
02 / 04 / 98 \\
02 / 04 / 98 \\
02 / 04 / 98 \\
11 / 13 / 97 \\
11 / 13 / 97 \\
11 / 13 / 97 \\
11 / 13 / 97 \\
11 / 13 / 97 \\
11 / 13 / 97 \\
11 / 13 / 97 \\
10 / 27 / 97 \\
10 / 27 / 97 \\
10 / 27 / 97 \\
10 / 27 / 97 \\
10 / 27 / 97 \\
10 / 27 / 97 \\
10 / 27 / 97 \\
02 / 05 / 98 \\
02 / 05 / 98 \\
02 / 05 / 98 \\
02 / 05 / 98 \\
02 / 05 / 98 \\
02 / 05 / 98 \\
02 / 05 / 98 \\
10 / 27 / 97 \\
10 / 27 / 97 \\
10 / 27 / 97 \\
10 / 27 / 97 \\
10 / 27 / 97 \\
10 / 27 / 97 \\
10 / 27 / 97 \\
02 / 05 / 98\end{array}$ & 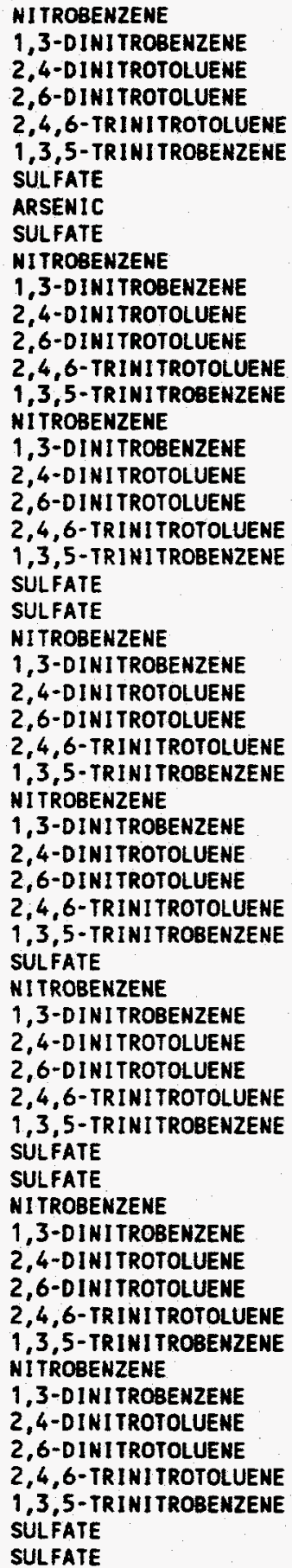 & 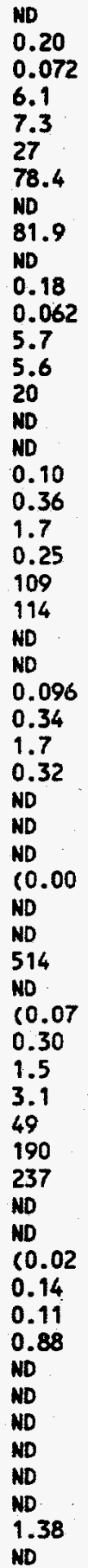 & $\begin{array}{l}0.030 \\
0.090 \\
0.030 \\
0.010 \\
0.030 \\
0.030 \\
5.0 \\
4.0 \\
2.50 \\
0.030 \\
0.090 \\
0.030 \\
0.010 \\
0.030 \\
0.030 \\
0.030 \\
0.090 \\
0.030 \\
0.010 \\
0.030 \\
0.030 \\
7.5 \\
5.00 \\
0.030 \\
0.090 \\
0.030 \\
0.010 \\
0.030 \\
0.030 \\
0.030 \\
0.090 \\
0.030 \\
0.010 \\
0.030 \\
0.030 \\
50.0 \\
0.030 \\
0.090 \\
0.030 \\
0.010 \\
0.030 \\
0.030 \\
1.00 \\
10.0 \\
0.030 \\
0.090 \\
0.030 \\
0.010 \\
0.030 \\
0.030 \\
0.030 \\
0.090 \\
0.030 \\
0.010 \\
0.030 \\
0.030 \\
1.00 \\
0.50\end{array}$ & 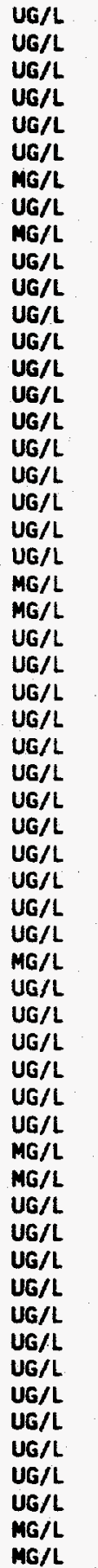 & $\begin{array}{l}\text { USATHAMA } \\
\text { USATHAMA } \\
\text { USATHAMA } \\
\text { USATHAMA } \\
\text { USATHAMA } \\
\text { USATHAMA } \\
\text { EPA } 300.0 \\
\text { EPA } 6010 A \\
\text { EPA } 300.0 \\
\text { USATHAMA } \\
\text { USATHAMA } \\
\text { USATHAMA } \\
\text { USATHAMA } \\
\text { USATHAMA } \\
\text { USATHAMA } \\
\text { USATHAMA } \\
\text { USATHAMA } \\
\text { USATHAMA } \\
\text { USATHAMA } \\
\text { USATHAMA } \\
\text { USATHAMA } \\
\text { EPA } 300.0 \\
\text { EPA } 300.0 \\
\text { USATHAMA } \\
\text { USATHAMA } \\
\text { USATHAMA } \\
\text { USATHAMA } \\
\text { USATHAMA } \\
\text { USATHAMA } \\
\text { USATHAMA } \\
\text { USATHAMA } \\
\text { USATHAMA } \\
\text { USATHAMA } \\
\text { USATHAMA } \\
\text { USATHAMA } \\
\text { EPA } 300.0 \\
\text { USATHAMA } \\
\text { USATHAMA } \\
\text { USATHAMA } \\
\text { USATHAMA } \\
\text { USATHAMA } \\
\text { USATHAMA } \\
\text { EPA } 300.0 \\
\text { EPA } 300.0 \\
\text { USATHAMA } \\
\text { USATHAMA } \\
\text { USATHAMA } \\
\text { USATHAMA } \\
\text { USATHAMA } \\
\text { USATHAMA } \\
\text { USATHAMA } \\
\text { USATHAMA } \\
\text { USATHAMA } \\
\text { USATHAMA } \\
\text { USATHAMA } \\
\text { USATHAMA } \\
\text { EPA }\end{array}$ \\
\hline
\end{tabular}




\begin{tabular}{|c|c|c|c|c|c|c|}
\hline USSRAP_ID & DATE_SAM & PARAMETER & CONC & DL & UNITS & METHOD \\
\hline $\begin{array}{l}G W-1007-0198 \\
G W-1007-0198 \\
G W-1007-0198 \\
G W-1007-0198 \\
G W-1007-0198 \\
G W-1007-0198 \\
G W-1008-0497 \\
G W-1008-0497 \\
G W-1008-0497 \\
G W-1008-0497 \\
G W-1008-0497 \\
G W-1008-0497 \\
G W-1008-0497 \\
G W-1008-0198 \\
G W-1008-0198 \\
G W-1008-0198 \\
G W-1008-0198 \\
G W-1008-0198 \\
G W-1008-0198 \\
G W-1008-0198 \\
G W-1009-0497 \\
G W-1009-0497 \\
G W-1009-0497 \\
G W-1009-0497 \\
G W-1009-0497 \\
G W-1009-0497 \\
G W-1009-0497 \\
G W-1009-0198 \\
G W-1009-0198 \\
G W-1009-0198 \\
G W-1009-0198 \\
G W-1009-0198 \\
G W-1009-0198 \\
G W-1009-0198 \\
G W-1012-5297 \\
G W-1012-5297 \\
G W-1012-5297 \\
G W-1012-5297 \\
G W-1012-5297 \\
G W-1012-5297 \\
G W-1012-5297 \\
G W-1012-5297 \\
G W-1012-5297 \\
G W-1012-5297 \\
G W-1012-5297 \\
G W-1012-5297 \\
G W-1012-5297 \\
G W-1012-5297 \\
G W-1013-0497 \\
G W-1013-0497 \\
G W-1013-0497 \\
G W-1013-0497 \\
G W-1013-0497 \\
G W-1013-0497 \\
G W-1013-0497 \\
G W-1013-0497 \\
G W-1013-0497 \\
G W-1013-0497 \\
G W-1013-0497 \\
G W-1013-0497 \\
G W-1013-0198\end{array}$ & $\begin{array}{l}02 / 05 / 98 \\
02 / 05 / 98 \\
02 / 05 / 98 \\
02 / 05 / 98 \\
02 / 05 / 98 \\
02 / 05 / 98 \\
10 / 27 / 97 \\
10 / 27 / 97 \\
10 / 27 / 97 \\
10 / 27 / 97 \\
10 / 27 / 97 \\
10 / 27 / 97 \\
10 / 27 / 97 \\
02 / 05 / 98 \\
02 / 05 / 98 \\
02 / 05 / 98 \\
02 / 05 / 98 \\
02 / 05 / 98 \\
02 / 05 / 98 \\
02 / 05 / 98 \\
10 / 27 / 97 \\
10 / 27 / 97 \\
10 / 27 / 97 \\
10 / 27 / 97 \\
10 / 27 / 97 \\
10 / 27 / 97 \\
10 / 27 / 97 \\
02 / 05 / 98 \\
02 / 05 / 98 \\
02 / 05 / 98 \\
02 / 05 / 98 \\
02 / 05 / 98 \\
02 / 05 / 98 \\
02 / 05 / 98 \\
10 / 20 / 97 \\
10 / 20 / 97 \\
10 / 20 / 97 \\
10 / 20 / 97 \\
10 / 20 / 97 \\
10 / 20 / 97 \\
10 / 20 / 97 \\
10 / 20 / 97 \\
10 / 20 / 97 \\
10 / 20 / 97 \\
10 / 20 / 97 \\
10 / 20 / 97 \\
10 / 20 / 97 \\
10 / 20 / 97 \\
10 / 23 / 97 \\
10 / 23 / 97 \\
10 / 23 / 97 \\
10 / 23 / 97 \\
10 / 23 / 97 \\
10 / 23 / 97 \\
10 / 23 / 97 \\
10 / 23 / 97 \\
10 / 23 / 97 \\
10 / 23 / 97 \\
10 / 23 / 97 \\
10 / 23 / 97 \\
02 / 09 / 98\end{array}$ & 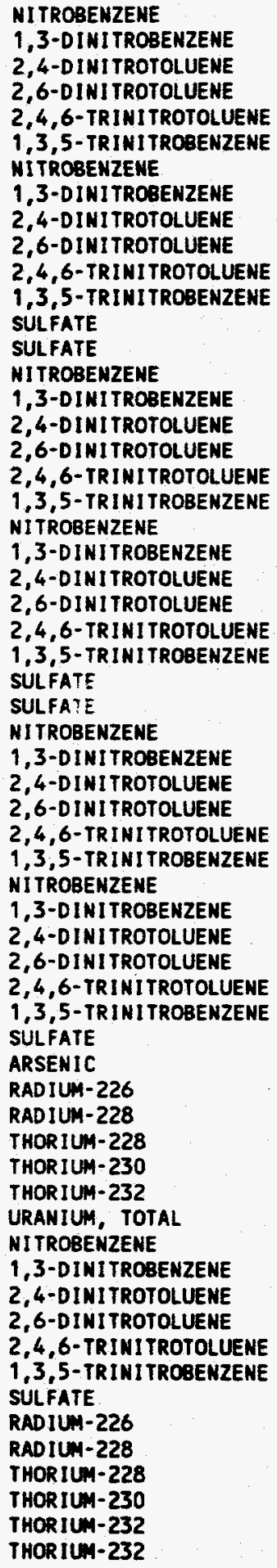 & 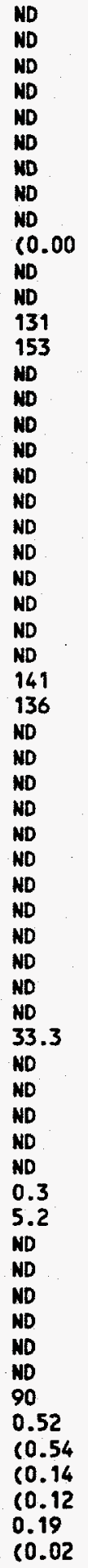 & $\begin{array}{l}0.030 \\
0.090 \\
0.030 \\
0.010 \\
0.030 \\
0.030 \\
0.030 \\
0.090 \\
0.030 \\
0.010 \\
0.030 \\
0.030 \\
1.00 \\
5.00 \\
0.030 \\
0.090 \\
0.030 \\
0.010 \\
0.030 \\
0.030 \\
0.030 \\
0.090 \\
0.030 \\
0.010 \\
0.030 \\
0.030 \\
1.00 \\
5.00 \\
0.030 \\
0.090 \\
0.030 \\
0.010 \\
0.030 \\
0.030 \\
0.030 \\
0.090 \\
0.030 \\
0.010 \\
0.030 \\
0.030 \\
2.5 \\
4.0 \\
0.7 \\
2 \\
0.8 \\
0.4 \\
0.2 \\
0.2 \\
0.030 \\
0.090 \\
0.030 \\
0.010 \\
0.030 \\
0.030 \\
0.030 \\
0.24 \\
0.69 \\
0.34 \\
0.13 \\
0.12 \\
0.056\end{array}$ & $\begin{array}{l}U G / L \\
U G / L \\
U G / L \\
U G / L \\
U G / L \\
U G / L \\
U G / L \\
U G / L \\
U G / L \\
U G / L \\
U G / L \\
U G / L \\
M G / L \\
\text { UG/L } \\
U G / L \\
U G / L \\
U G / L \\
U G / L \\
U G / L \\
U G / L \\
U G / L \\
U G / L \\
U G / L \\
U G / L \\
U G / L \\
U G / L \\
M G / L \\
M G / L \\
U G / L \\
U G / L \\
U G / L \\
U G / L \\
U G / L \\
U G / L \\
U G / L \\
U G / L \\
U G / L \\
U G / L \\
U G / L \\
U G / L \\
M G / L \\
U G / L \\
P C I / L \\
P C I / L \\
P C I / L \\
P C I / L \\
P C I / L \\
P C I / L \\
U G / L \\
U G / L \\
U G / L \\
U G / L \\
U G / L \\
U G / L \\
M G / L \\
P C I / L \\
P C I / L \\
P C I / L \\
P C I / L \\
P C I / L \\
P C I / L \\
\end{array}$ & $\begin{array}{l}\text { USATHAMA } \\
\text { USATHAMA } \\
\text { USATHAMA } \\
\text { USATHAMA } \\
\text { USATHAMA } \\
\text { USATHAMA } \\
\text { USATHAMA } \\
\text { USATHAMA } \\
\text { USATHAMA } \\
\text { USATHAMA } \\
\text { USATHAMA } \\
\text { USATHAMA } \\
\text { EPA } 300.0 \\
\text { EPA } 300.0 \\
\text { USATHAMA } \\
\text { USATHAMA } \\
\text { USATHAMA } \\
\text { USATHAMA } \\
\text { USATHAMA } \\
\text { USATHAMA } \\
\text { USATHAMA } \\
\text { USATHAMA } \\
\text { USATHAMA } \\
\text { USATHAMA } \\
\text { USATHAMA } \\
\text { USATHAMA } \\
\text { EPA } 300.0 \\
\text { EPA } 300.0 \\
\text { USATHAMA } \\
\text { USATHAMA } \\
\text { USATHAMA } \\
\text { USATHAMA } \\
\text { USATHAMA } \\
\text { USATHAMA } \\
\text { USATHAMA } \\
\text { USATHAMA } \\
\text { USATHAMA } \\
\text { USATHAMA } \\
\text { USATHAMA } \\
\text { USATHAMA } \\
\text { EPA } 300.0 \\
\text { EPA 6010A } \\
\text { SM-705 } \\
\text { PERC/BROOKS } \\
\text { USAEC } \\
\text { USAEC } \\
\text { USAEC } \\
\text { ASTM D2907 } \\
\text { USATHAMA } \\
\text { USATHAMA } \\
\text { USATHAMA } \\
\text { USATHAMA } \\
\text { USATHAMA } \\
\text { USATHAMA } \\
\text { EPA } 300.0 \\
\text { EPA 903.1 } \\
\text { EPA 904.0 } \\
\text { EPA 907.0 } \\
\text { EPA 907.0 } \\
\text { EPA 907.0 } \\
\text { UAS-NS-3004 }\end{array}$ \\
\hline
\end{tabular}




\begin{tabular}{|c|c|c|c|c|c|c|}
\hline WSSRAP_ID & DATE_SAM & PARAMETER & CONC & $\mathrm{DL}$ & UNITS & METHOD \\
\hline 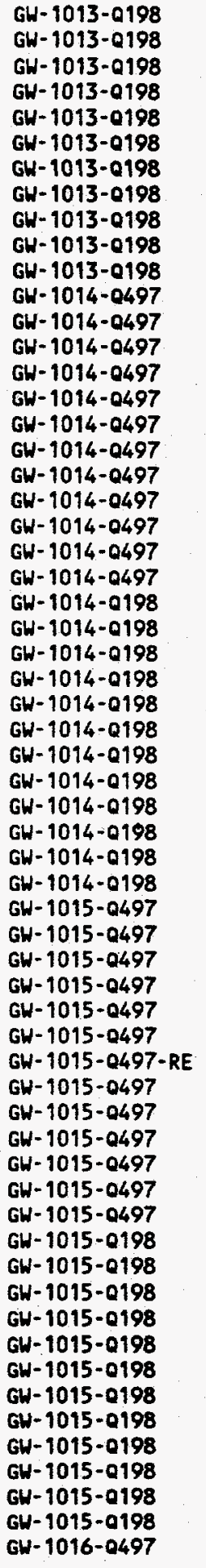 & $\begin{array}{l}02 / 09 / 98 \\
02 / 09 / 98 \\
02 / 09 / 98 \\
02 / 09 / 98 \\
02 / 09 / 98 \\
02 / 09 / 98 \\
02 / 09 / 98 \\
02 / 09 / 98 \\
02 / 09 / 98 \\
02 / 09 / 98 \\
02 / 09 / 98 \\
10 / 23 / 97 \\
10 / 23 / 97 \\
10 / 23 / 97 \\
10 / 23 / 97 \\
10 / 23 / 97 \\
10 / 23 / 97 \\
10 / 23 / 97 \\
10 / 23 / 97 \\
10 / 23 / 97 \\
10 / 23 / 97 \\
10 / 23 / 97 \\
10 / 23 / 97 \\
02 / 09 / 98 \\
02 / 09 / 98 \\
02 / 09 / 98 \\
02 / 09 / 98 \\
02 / 09 / 98 \\
02 / 09 / 98 \\
02 / 09 / 98 \\
02 / 09 / 98 \\
02 / 09 / 98 \\
02 / 09 / 98 \\
02 / 09 / 98 \\
02 / 09 / 98 \\
10 / 28 / 97 \\
10 / 28 / 97 \\
10 / 28 / 97 \\
10 / 28 / 97 \\
10 / 28 / 97 \\
10 / 28 / 97 \\
10 / 28 / 97 \\
10 / 28 / 97 \\
10 / 28 / 97 \\
10 / 28 / 97 \\
10 / 28 / 97 \\
10 / 28 / 97 \\
10 / 28 / 97 \\
02 / 09 / 98 \\
02 / 09 / 98 \\
02 / 09 / 98 \\
02 / 09 / 98 \\
02 / 09 / 98 \\
02 / 09 / 98 \\
02 / 09 / 98 \\
02 / 09 / 98 \\
02 / 09 / 98 \\
02 / 09 / 98 \\
02 / 09 / 98 \\
02 / 09 / 98 \\
10 / 28 / 97\end{array}$ & 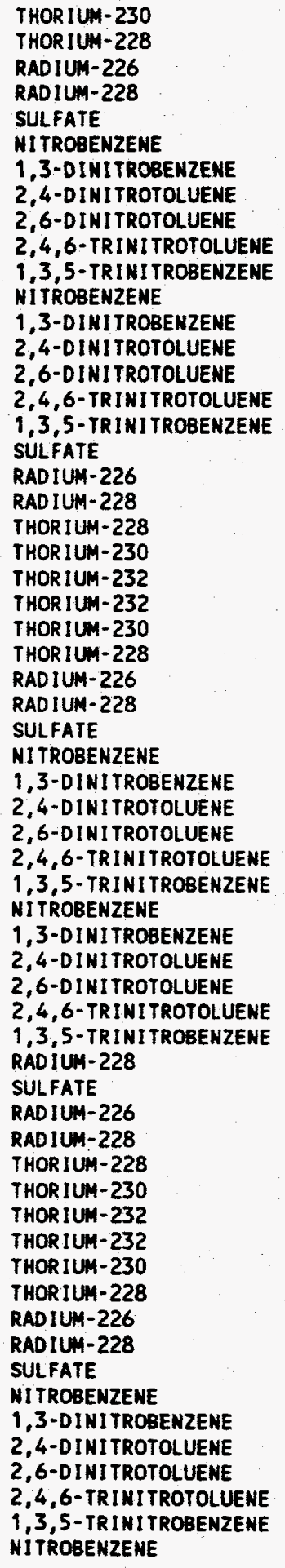 & $\begin{array}{l}10.09 \\
C 0.03 \\
0.600 \\
0.576 \\
92.4 \\
\text { ND } \\
\text { ND } \\
C 0.02 \\
C 0.00 \\
\text { ND } \\
\text { ND } \\
\text { ND } \\
\text { ND } \\
\text { ND } \\
\text { ND } \\
\text { ND } \\
\text { ND } \\
94.9 \\
C 0.14 \\
0.95 \\
C 0.17 \\
\text { ND } \\
C 0.00 \\
C 0.00 \\
C 0.03 \\
0.120 \\
0.236 \\
0.364 \\
99.1 \\
\text { ND } \\
\text { ND } \\
\text { ND } \\
\text { ND } \\
\text { ND } \\
\text { ND } \\
\text { ND } \\
0.13 \\
60.02 \\
0.18 \\
1.2 \\
2.3 \\
11.21 \\
145 \\
1.11 \\
26.7 \\
\text { ND } \\
0.230 \\
10.02 \\
10.08 \\
0.142 \\
0.143 \\
0.162 \\
10.23 \\
112 \\
\text { ND } \\
0.16 \\
10.02 \\
0.17 \\
1.4 \\
2.5 \\
\text { ND } \\
0\end{array}$ & 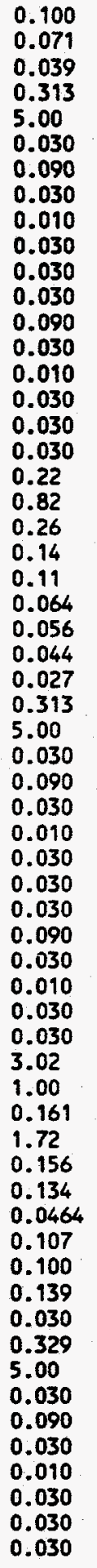 & $\begin{array}{l}P C I / L \\
P C I / L \\
P C I / L \\
P C I / L \\
\text { UG/L } \\
U G / L \\
U G / L \\
U G / L \\
U G / L \\
U G / L \\
U G / L \\
U G / L \\
U G / L \\
U G / L \\
U G / L \\
U G / L \\
U G / L \\
M G / L \\
P C I / L \\
P C I / L \\
P C I / L \\
P C I / L \\
P C I / L \\
P C I / L \\
P C I / L \\
P C I / L \\
P C I / L \\
P C I / L \\
M G / L \\
U G / L \\
U G / L \\
U G / L \\
U G / L \\
U G / L \\
U G / L \\
U G / L \\
U G / L \\
U G / L \\
U G / L \\
U G / L \\
U G / L \\
P C I / L \\
M G / L \\
P C I / L \\
P C I / L \\
P C I / L \\
P C I / L \\
P C I / L \\
P C I / L \\
P C I / L \\
P C I / L \\
P C I / L \\
P C I / L \\
\text { MG/L} \\
U G / L \\
U G / L \\
U G / L \\
U G / L \\
U G / L \\
U G / L \\
U G / L \\
\end{array}$ & 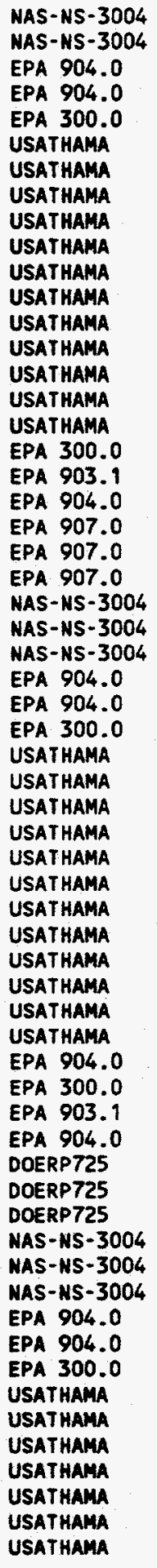 \\
\hline
\end{tabular}




\begin{tabular}{|c|c|c|c|c|c|c|}
\hline HSSRAP_ID & DATE_SAM & PARAMETER & CONC & DL & UNITS & METHOO \\
\hline 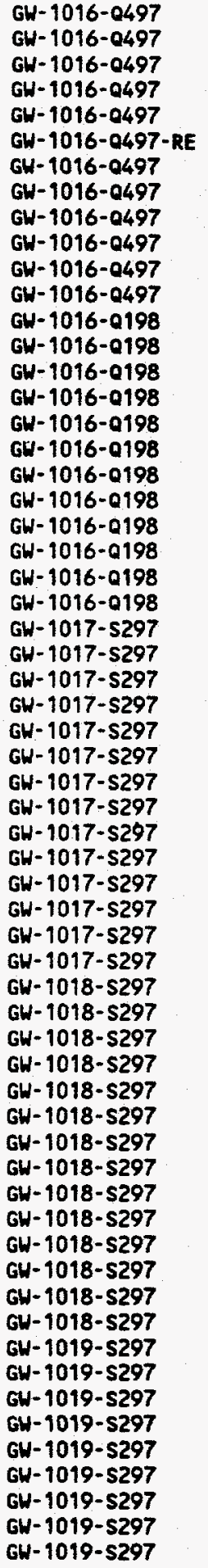 & $\begin{array}{l}10 / 28 / 97 \\
10 / 28 / 97 \\
10 / 28 / 97 \\
10 / 28 / 97 \\
10 / 28 / 97 \\
10 / 28 / 97 \\
10 / 28 / 97 \\
10 / 28 / 97 \\
10 / 28 / 97 \\
10 / 28 / 97 \\
10 / 28 / 97 \\
10 / 28 / 97 \\
02 / 09 / 98 \\
02 / 09 / 98 \\
02 / 09 / 98 \\
02 / 09 / 98 \\
02 / 09 / 98 \\
02 / 09 / 98 \\
02 / 09 / 98 \\
02 / 09 / 98 \\
02 / 09 / 98 \\
02 / 09 / 98 \\
02 / 09 / 98 \\
02 / 09 / 98 \\
12 / 08 / 97 \\
12 / 08 / 97 \\
12 / 08 / 97 \\
12 / 08 / 97 \\
12 / 08 / 97 \\
12 / 08 / 97 \\
12 / 08 / 97 \\
12 / 08 / 97 \\
12 / 08 / 97 \\
12 / 08 / 97 \\
12 / 08 / 97 \\
12 / 08 / 97 \\
12 / 08 / 97 \\
12 / 08 / 97 \\
12 / 08 / 97 \\
12 / 08 / 97 \\
12 / 08 / 97 \\
12 / 08 / 97 \\
12 / 08 / 97 \\
12 / 08 / 97 \\
12 / 08 / 97 \\
12 / 08 / 97 \\
12 / 08 / 97 \\
12 / 08 / 97 \\
12 / 08 / 97 \\
12 / 08 / 97 \\
12 / 08 / 97 \\
12 / 08 / 97 \\
12 / 09 / 97 \\
12 / 09 / 97 \\
12 / 09 / 97 \\
12 / 09 / 97 \\
12 / 09 / 97 \\
12 / 09 / 97 \\
12 / 09 / 97 \\
12 / 09 / 97 \\
12 / 09 / 97\end{array}$ & 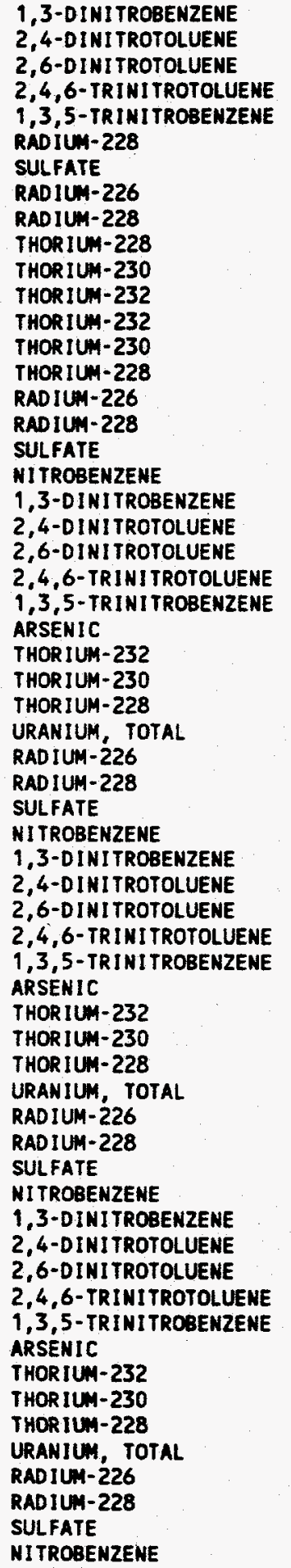 & $\begin{array}{l}\text { ND } \\
\text { ND } \\
0.019 \\
10.01 \\
10.02 \\
4.27 \\
140 \\
0.440 \\
9.36 \\
10.04 \\
0.400 \\
0.070 \\
10.01 \\
0.168 \\
0.112 \\
0.148 \\
C 0.27 \\
146 \\
\text { ND } \\
\text { ND } \\
\text { ND } \\
0.027 \\
0.090 \\
0.12 \\
110 \\
0.117 \\
0.169 \\
0.194 \\
\text { ND } \\
1.18 \\
1.61 \\
3.47 \\
\text { ND } \\
\text { ND } \\
\text { ND } \\
\text { ND } \\
\text { ND } \\
\text { ND } \\
95.6 \\
0.315 \\
0.392 \\
0.450 \\
\text { ND } \\
1.05 \\
1.62 \\
62.7 \\
\text { ND } \\
\text { ND } \\
\text { ND } \\
\text { ND } \\
\text { ND } \\
\text { ND } \\
80.6 \\
0.471 \\
0.554 \\
1.09 \\
\text { ND } \\
1.77 \\
2.46 \\
\text { ND } \\
\text { ND }\end{array}$ & $\begin{array}{l}0.090 \\
0.030 \\
0.010 \\
0.030 \\
0.030 \\
1.13 \\
1.00 \\
0.211 \\
1.76 \\
0.146 \\
0.125 \\
0.0483 \\
0.113 \\
0.09 \\
0.08 \\
0.04 \\
0.047 \\
0.325 \\
5.00 \\
0.030 \\
0.090 \\
0.030 \\
0.010 \\
0.030 \\
0.030 \\
0\end{array}$ & 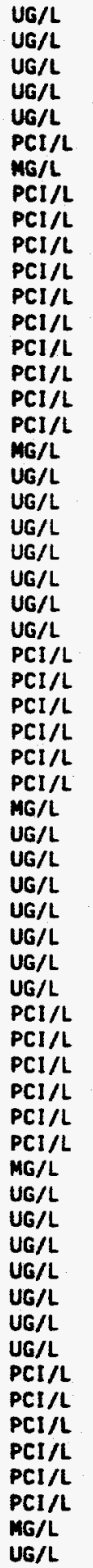 & 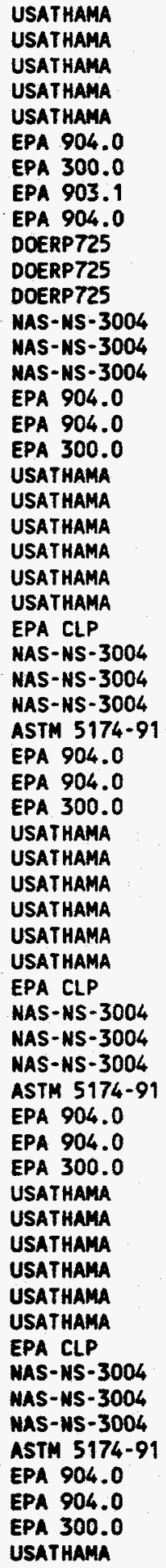 \\
\hline
\end{tabular}




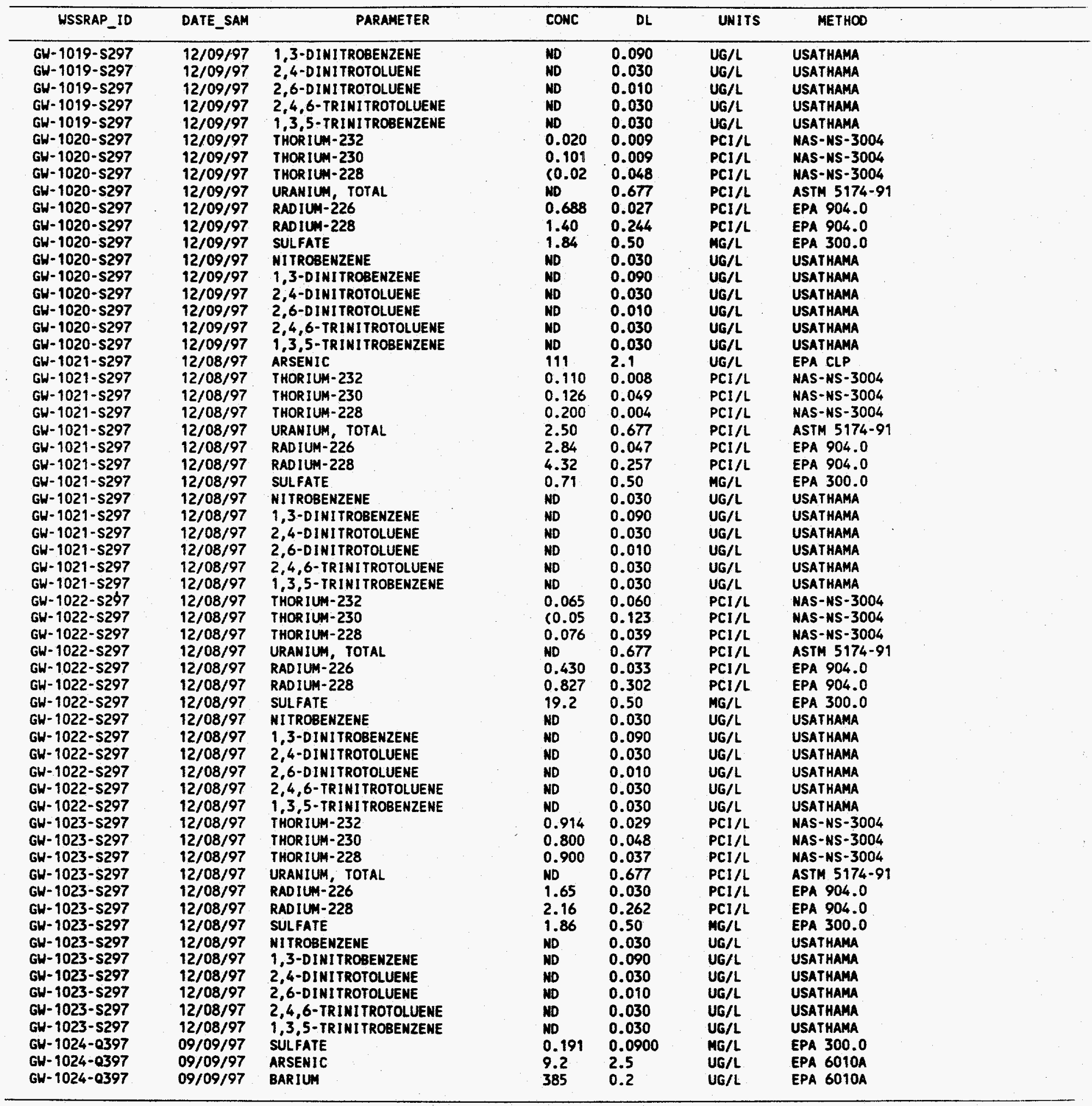




\begin{tabular}{|c|c|c|c|c|c|c|}
\hline WSSRAP_10 & DATE_SAM & PARAMETER & CONC & $D L$ & UNITS & METHOD \\
\hline 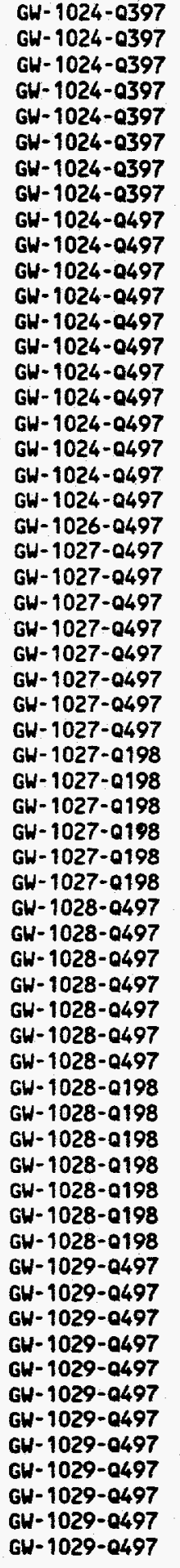 & $\begin{array}{l}09 / 09 / 97 \\
09 / 09 / 97 \\
09 / 09 / 97 \\
09 / 09 / 97 \\
09 / 09 / 97 \\
09 / 09 / 97 \\
09 / 09 / 97 \\
09 / 09 / 97 \\
10 / 28 / 97 \\
10 / 28 / 97 \\
10 / 28 / 97 \\
10 / 28 / 97 \\
10 / 28 / 97 \\
10 / 28 / 97 \\
10 / 28 / 97 \\
10 / 28 / 97 \\
10 / 28 / 97 \\
10 / 28 / 97 \\
10 / 28 / 97 \\
10 / 28 / 97 \\
10 / 20 / 97 \\
10 / 20 / 97 \\
10 / 20 / 97 \\
10 / 20 / 97 \\
10 / 20 / 97 \\
10 / 20 / 97 \\
10 / 20 / 97 \\
10 / 20 / 97 \\
10 / 20 / 97 \\
01 / 27 / 98 \\
01 / 27 / 98 \\
01 / 27 / 98 \\
01 / 27 / 98 \\
01 / 27 / 98 \\
01 / 27 / 98 \\
10 / 23 / 97 \\
10 / 23 / 97 \\
10 / 23 / 97 \\
10 / 23 / 97 \\
10 / 23 / 97 \\
10 / 23 / 97 \\
10 / 23 / 97 \\
02 / 09 / 98 \\
02 / 09 / 98 \\
02 / 09 / 98 \\
02 / 09 / 98 \\
02 / 09 / 98 \\
02 / 09 / 98 \\
02 / 09 / 98 \\
111 / 13 / 97 \\
11 / 13 / 97 \\
11 / 13 / 97 \\
11 / 13 / 97 \\
11113 / 97 \\
111 / 13 / 97 \\
11 / 13 / 97 \\
11 / 13 / 97 \\
11 / 13 / 97 \\
11 / 13 / 97 \\
11 / 13 / 97 \\
11 / 13 / 97\end{array}$ & 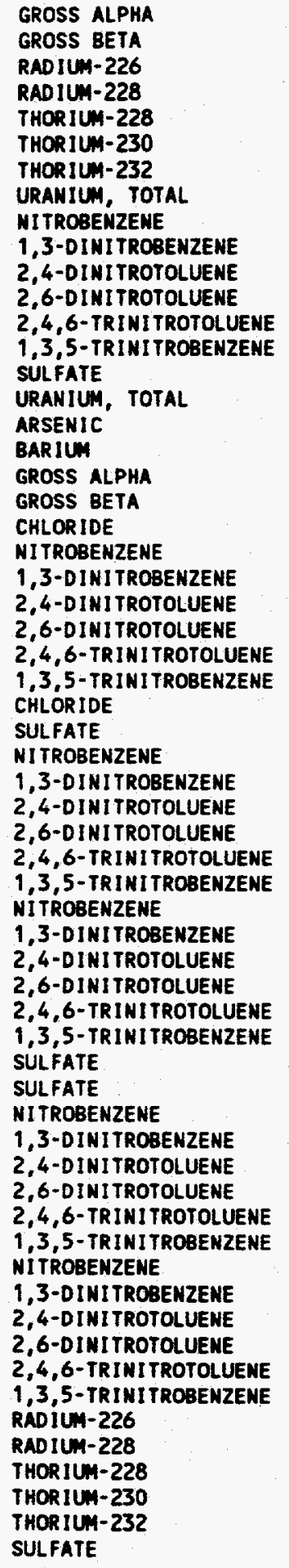 & 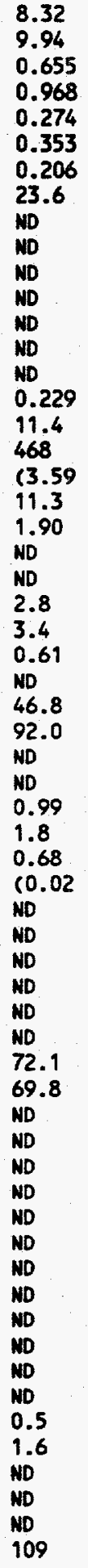 & 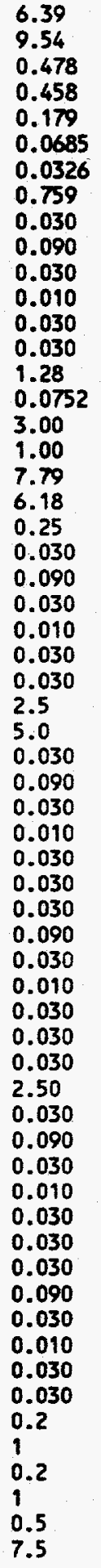 & $\begin{array}{l}P C I / L \\
P C I / L \\
P C I / L \\
P C I / L \\
P C I / L \\
P C I / L \\
P C I / L \\
P C I / L \\
U G / L \\
U G / L \\
U G / L \\
U G / L \\
U G / L \\
U G / L \\
M G / L \\
P C I / L \\
U G / L \\
U G / L \\
P C I / L \\
P C I / L \\
M G / L \\
U G / L \\
U G / L \\
U G / L \\
U G / L \\
U G / L \\
U G / L \\
M G / L \\
M G / L \\
U G / L \\
U G / L \\
U G / L \\
U G / L \\
U G / L \\
U G / L \\
U G / L \\
U G / L \\
U G / L \\
U G / L \\
U G / L \\
U G / L \\
M G / L \\
M G / L \\
U G / L \\
U G / L \\
U G / L \\
U G / L \\
U G / L \\
U G / L \\
U G / L \\
U G / L \\
U G / L \\
U G / L \\
U G / L \\
U G / L \\
P C I / L \\
P C I / L \\
P C I / L \\
P C I / L \\
P C I / L \\
M G / L \\
\text { UG }\end{array}$ & $\begin{array}{l}\text { EPA } 900.0 \\
\text { EPA } 900.0 \\
\text { EPA } 903.1 \\
\text { EPA } 904.0 \\
\text { HASL } 300 \\
\text { HASL } 300 \\
\text { HASL } 300 \\
\text { ASTM } 5174-91 \\
\text { USATHAMA } \\
\text { USATHAMA } \\
\text { USATHAMA } \\
\text { USATHAMA } \\
\text { USATHAMA } \\
\text { USATHAMA } \\
\text { EPA } 300.0 \\
\text { EPA } 6020 \\
\text { EPA CLP } \\
\text { EPA CLP } \\
\text { EPA 900.0 } \\
\text { EPA 900.0 } \\
\text { EPA } 300.0 \\
\text { USATHAMA } \\
\text { USATHAMA } \\
\text { USATHAMA } \\
\text { USATHAMA } \\
\text { USATHAMA } \\
\text { USATHAMA } \\
\text { EPA 300.0 } \\
\text { EPA } 300.0 \\
\text { USATHAMA } \\
\text { USATHAMA } \\
\text { USATHAMA } \\
\text { USATHAMA } \\
\text { USATHAMA } \\
\text { USATHAMA } \\
\text { USATHAMA } \\
\text { USATHAMA } \\
\text { USATHAMA } \\
\text { USATHAMA } \\
\text { USATHAMA } \\
\text { USATHAMA } \\
\text { EPA } 300.0 \\
\text { EPA } 300.0 \\
\text { USATHAMA } \\
\text { USATHAMA } \\
\text { USATHAMA } \\
\text { USATHAMA } \\
\text { USATHAMA } \\
\text { USATHAMA } \\
\text { USATHAMA } \\
\text { USATHAMA } \\
\text { USATHAMA } \\
\text { USATHAMA } \\
\text { USATHAMA } \\
\text { USATHAMA } \\
\text { SM-705 } \\
\text { PERC/BROOKS } \\
\text { USAEC } \\
\text { USAEC } \\
\text { USAEC } \\
\text { EPA } 300.0 \\
\end{array}$ \\
\hline
\end{tabular}




\begin{tabular}{|c|c|c|c|c|c|c|}
\hline HSSRAP_ID & DATE_SAM & PARAMETER & CONC & DL & UNITS & METHOO \\
\hline 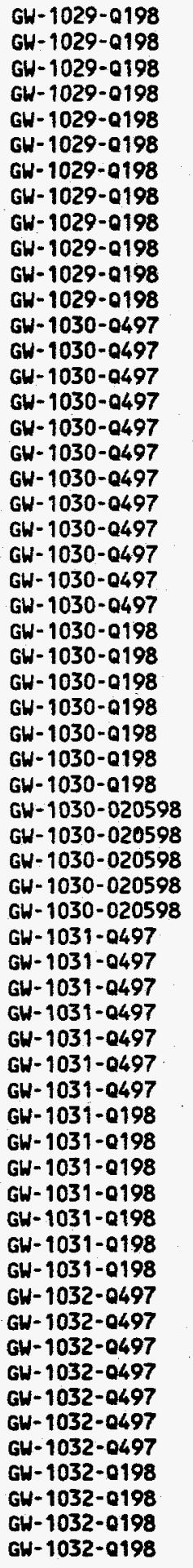 & $\begin{array}{l}02 / 04 / 98 \\
02 / 04 / 98 \\
02 / 04 / 98 \\
02 / 04 / 98 \\
02 / 04 / 98 \\
02 / 04 / 98 \\
02 / 04 / 98 \\
02 / 04 / 98 \\
02 / 04 / 98 \\
02 / 04 / 98 \\
02 / 04 / 98 \\
02 / 04 / 98 \\
11 / 13 / 97 \\
11 / 13 / 97 \\
11 / 13 / 97 \\
11 / 13 / 97 \\
11 / 13 / 97 \\
11 / 13 / 97 \\
11 / 13 / 97 \\
11 / 13 / 97 \\
11 / 13 / 97 \\
11 / 13 / 97 \\
11 / 13 / 97 \\
11 / 13 / 97 \\
02 / 04 / 98 \\
02 / 04 / 98 \\
02 / 04 / 98 \\
02 / 04 / 98 \\
02 / 04 / 98 \\
02 / 04 / 98 \\
02 / 04 / 98 \\
02 / 05 / 98 \\
02 / 05 / 98 \\
02 / 05 / 98 \\
02 / 05 / 98 \\
02 / 05 / 98 \\
10 / 23 / 97 \\
10 / 23 / 97 \\
10 / 23 / 97 \\
10 / 23 / 97 \\
10 / 23 / 97 \\
10 / 23 / 97 \\
10 / 23 / 97 \\
02 / 09 / 98 \\
02 / 09 / 98 \\
02 / 09 / 98 \\
02 / 09 / 98 \\
02 / 09 / 98 \\
02 / 09 / 98 \\
02 / 09 / 98 \\
10 / 27 / 97 \\
10 / 27 / 97 \\
10 / 27 / 97 \\
10 / 27 / 97 \\
10 / 27 / 97 \\
10 / 27 / 97 \\
10 / 27 / 97 \\
02 / 05 / 98 \\
02 / 05 / 98 \\
02 / 05 / 98 \\
02 / 05 / 98\end{array}$ & 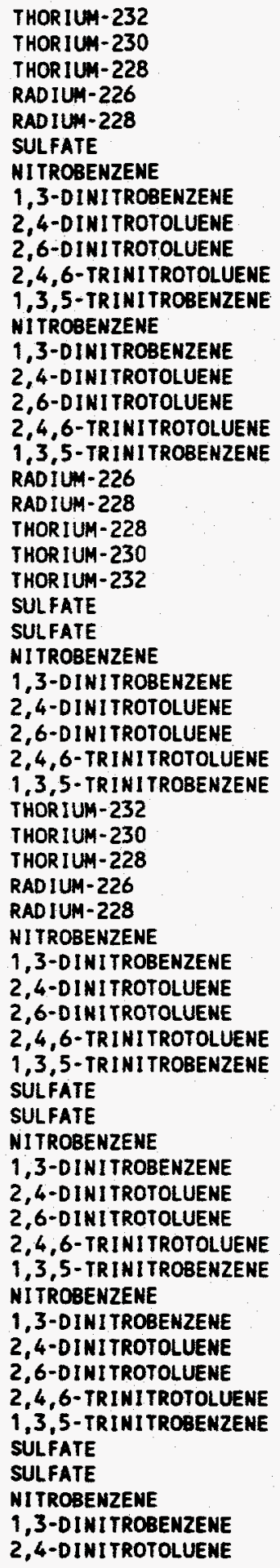 & 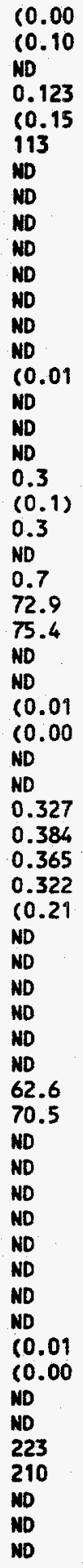 & $\begin{array}{l}0.124 \\
0.109 \\
0.233 \\
0.054 \\
0.288 \\
5.00 \\
0.030 \\
0.090 \\
0.030 \\
0.010 \\
0.030 \\
0.030 \\
0.030 \\
0.090 \\
0.030 \\
0.010 \\
0.030 \\
0.030 \\
0.2 \\
1 \\
0.2 \\
1 \\
0.5 \\
5.0 \\
2.50 \\
0.030 \\
0.090 \\
0.030 \\
0.010 \\
0.030 \\
0.030 \\
0.083 \\
0.116 \\
0.148 \\
0.070 \\
0.371 \\
0.030 \\
0.090 \\
0.030 \\
0.010 \\
0.030 \\
0.030 \\
0.030 \\
2.50 \\
0.030 \\
0.090 \\
0.030 \\
0.010 \\
0.030 \\
0.030 \\
0.030 \\
0.090 \\
0.030 \\
0.010 \\
0.030 \\
0.030 \\
1.00 \\
10.0 \\
0.030 \\
0.090 \\
0.030\end{array}$ & 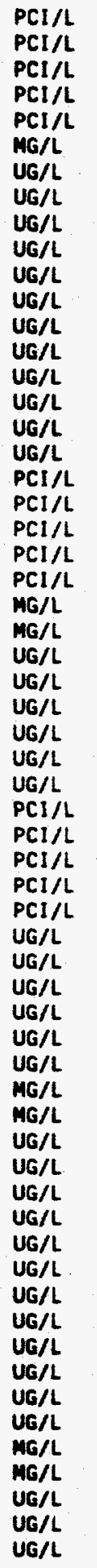 & $\begin{array}{l}\text { NAS-NS-3004 } \\
\text { NAS-NS-3004 } \\
\text { NAS-NS-3004 } \\
\text { EPA } 904.0 \\
\text { EPA } 904.0 \\
\text { EPA } 300.0 \\
\text { USATHAMA } \\
\text { USATHAMA } \\
\text { USATHAMA } \\
\text { USATHAMA } \\
\text { USATHAMA } \\
\text { USATHAMA } \\
\text { USATHAMA } \\
\text { USATHAMA } \\
\text { USATHAMA } \\
\text { USATHAMA } \\
\text { USATHAMA } \\
\text { USATHAMA } \\
\text { SH-705 } \\
\text { PERC/BROOKS } \\
\text { USAEC } \\
\text { USAEC } \\
\text { USAEC } \\
\text { EPA } 300.0 \\
\text { EPA } 300.0 \\
\text { USATHAMA } \\
\text { USATHAMA } \\
\text { USATHAMA } \\
\text { USATHAMA } \\
\text { USATHAMA } \\
\text { USATHAMA } \\
\text { NAS-NS-3004 } \\
\text { NAS-NS-3004 } \\
\text { NAS-NS-3004 } \\
\text { EPA 904.0 } \\
\text { EPA 904.0 } \\
\text { USATHAMA } \\
\text { USATHAMA } \\
\text { USATHAMA } \\
\text { USATHAMA } \\
\text { USATHAMA } \\
\text { USATHAMA } \\
\text { EPA } 300.0 \\
\text { EPA } 300.0 \\
\text { USATHAMA } \\
\text { USATHAMA } \\
\text { USATHAMA } \\
\text { USATHAMA } \\
\text { USATHAMA } \\
\text { USATHAMA } \\
\text { USATHAMA } \\
\text { USATHAMA } \\
\text { USATHAMA } \\
\text { USATHAMA } \\
\text { USATHAMA } \\
\text { USATHAMA } \\
\text { EPA } 300.0 \\
\text { EPA } 300.0 \\
\text { USATHAMA } \\
\text { USATHAMA } \\
\text { USATHAMA } \\
\text { USA }\end{array}$ \\
\hline
\end{tabular}




\begin{tabular}{|c|c|c|c|c|c|c|}
\hline WSSRAP_ID & DATE_SAM & PARAMETER & CONC & $D L$ & UNITS & METHOO \\
\hline 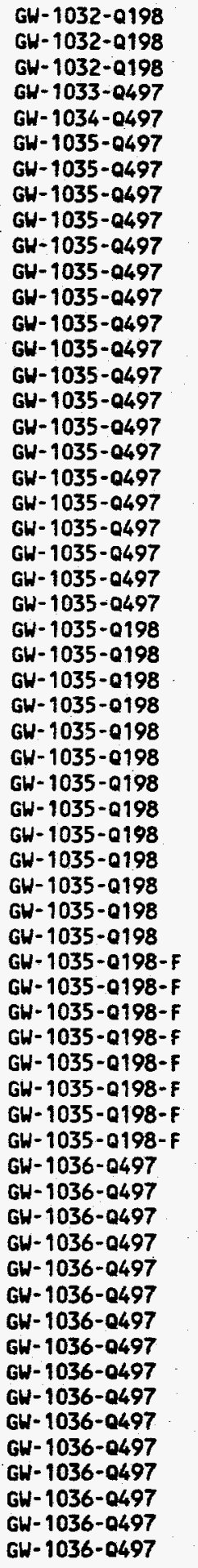 & $\begin{array}{l}02 / 05 / 98 \\
02 / 05 / 98 \\
02 / 05 / 98 \\
12 / 09 / 97 \\
10 / 20 / 97 \\
10 / 21 / 97 \\
10 / 21 / 97 \\
10 / 21 / 97 \\
10 / 21 / 97 \\
10 / 21 / 97 \\
10 / 21 / 97 \\
10 / 21 / 97 \\
10 / 21 / 97 \\
10 / 21 / 97 \\
10 / 21 / 97 \\
10 / 21 / 97 \\
10 / 21 / 97 \\
10 / 21 / 97 \\
10 / 21 / 97 \\
10 / 21 / 97 \\
10 / 21 / 97 \\
10 / 21 / 97 \\
10 / 21 / 97 \\
10 / 21 / 97 \\
01 / 23 / 98 \\
01 / 23 / 98 \\
01 / 23 / 98 \\
01 / 23 / 98 \\
01 / 23 / 98 \\
01 / 23 / 98 \\
01 / 23 / 98 \\
01 / 23 / 98 \\
01 / 23 / 98 \\
01 / 23 / 98 \\
01 / 23 / 98 \\
01 / 23 / 98 \\
01 / 23 / 98 \\
01 / 23 / 98 \\
01 / 23 / 98 \\
01 / 23 / 98 \\
01 / 23 / 98 \\
01 / 23 / 98 \\
01 / 23 / 98 \\
01 / 23 / 98 \\
01 / 23 / 98 \\
10 / 21 / 97 \\
10 / 21 / 97 \\
10 / 21 / 97 \\
10 / 21197 \\
10 / 21 / 97 \\
10 / 21 / 97 \\
10 / 21 / 97 \\
10 / 21197 \\
10 / 21 / 97 \\
10 / 21 / 97 \\
10 / 21 / 97 \\
10 / 21 / 97 \\
10 / 21197 \\
10 / 21 / 97 \\
10 / 21 / 97 \\
10 / 21 / 97\end{array}$ & 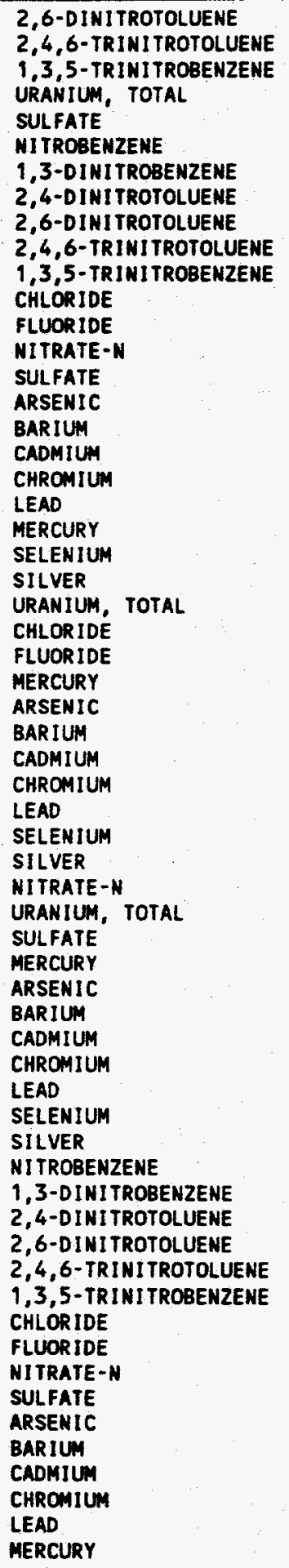 & $\begin{array}{l}\text { Co.00 } \\
\text { ND } \\
\text { ND } \\
2.56 \\
\text { S1.0 } \\
\text { ND } \\
\text { ND } \\
\text { ND } \\
\text { ND } \\
\text { ND } \\
20.2 \\
0.16 \\
0.134 \\
47.2 \\
\text { ND } \\
305 \\
\text { ND } \\
6.0 \\
\text { ND } \\
\text { ND } \\
\text { ND } \\
1.4 \\
1.8 \\
19.0 \\
0.24 \\
\text { ND } \\
\text { ND } \\
252 \\
\text { ND } \\
3.5 \\
\text { ND } \\
\text { ND } \\
\text { ND } \\
0.13 \\
\text { ND } \\
42.3 \\
\text { ND } \\
\text { ND } \\
248 \\
\text { ND } \\
\text { ND } \\
\text { ND } \\
\text { ND } \\
\text { ND } \\
\text { ND } \\
\text { ND } \\
\text { ND } \\
\text { ND } \\
\text { ND } \\
\text { ND } \\
119 \\
0.19 \\
0.092 \\
61.5 \\
\text { ND } \\
\text { N66 } \\
\text { ND } \\
6.4 \\
2.3 \\
\text { ND }\end{array}$ & $\begin{array}{l}0.010 \\
0.030 \\
0.030 \\
0.677 \\
5.0 \\
0.030 \\
0.090 \\
0.030 \\
0.010 \\
0.030 \\
0.030 \\
2.5 \\
0.05 \\
0.020 \\
5.0 \\
4.0 \\
2.0 \\
2.0 \\
2.0 \\
2.0 \\
0.10 \\
5.0 \\
1.0 \\
0.2 \\
1.00 \\
0.10 \\
0.10 \\
1.3 \\
0.40 \\
3.3 \\
2.7 \\
0.90 \\
2.2 \\
6.0 \\
0.050 \\
0.677 \\
2.50 \\
0.10 \\
1.3 \\
0.40 \\
3.3 \\
2.7 \\
0.90 \\
2.2 \\
6.0 \\
0.030 \\
0.090 \\
0.030 \\
0.010 \\
0.030 \\
0.030 \\
5.0 \\
0.05 \\
0.020 \\
5.0 \\
4.0 \\
2.0 \\
2.0 \\
2.0 \\
2.0 \\
0.10\end{array}$ & 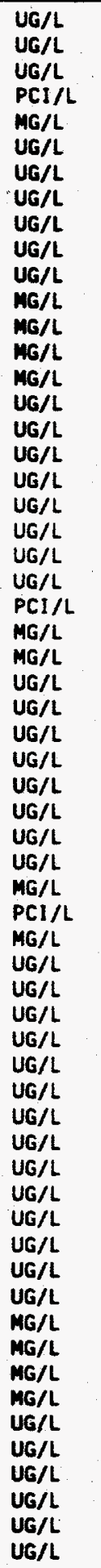 & $\begin{array}{l}\text { USATHAMA } \\
\text { USATHAMA } \\
\text { USATHAMA } \\
\text { ASTM } 5174-91 \\
\text { EPA } 300.0 \\
\text { USATHAMA } \\
\text { USATHAMA } \\
\text { USATHAMA } \\
\text { USATHAMA } \\
\text { USATHAMA } \\
\text { USATHAMA } \\
\text { EPA } 300.0 \\
\text { EPA } 340 \\
\text { EPA } 353.1 \\
\text { EPA } 300.0 \\
\text { EPA } 6010 A \\
\text { EPA } 6010 A \\
\text { EPA } 6010 A \\
\text { EPA } 6010 A \\
\text { EPA } 6010 A \\
\text { EPA } 7470 A \\
\text { EPA } 6010 A \\
\text { EPA } 6010 A \\
\text { ASTM D2907 } \\
\text { EPA } 300.0 \\
\text { EPA } 300.0 \\
\text { EPA CLP } \\
\text { EPA CLP } \\
\text { EPA CLP } \\
\text { EPA CLP } \\
\text { EPA CLP } \\
\text { EPA CLP } \\
\text { EPA CLP } \\
\text { EPA CLP } \\
\text { EPA } 353.1 \\
\text { ASTM } 5174-91 \\
\text { EPA } 300.0 \\
\text { EPA CLP } \\
\text { EPA CLP } \\
\text { EPA CLP } \\
\text { EPA CLP } \\
\text { EPA CLP } \\
\text { EPA CLP } \\
\text { EPA CLP } \\
\text { EPA CLP } \\
\text { USATHAMA } \\
\text { USATHAMA } \\
\text { USATHAMA } \\
\text { USATHAMA } \\
\text { USATHAMA } \\
\text { USATHAMA } \\
\text { EPA } 300.0 \\
\text { EPA } 340 \\
\text { EPA } 353.1 \\
\text { EPA } 300.0 \\
\text { EPA } 6010 A \\
\text { EPA } 6010 A \\
\text { EPA } 6010 A \\
\text { EPA } 6010 A \\
\text { EPA } 6010 A \\
\text { EPA } 7470 A \\
\end{array}$ \\
\hline
\end{tabular}




\begin{tabular}{|c|c|c|c|c|c|c|}
\hline WSSRAP_ID & DATE_SAM & PARAME TER & CONC & $\mathrm{OL}$ & UNITS & METHOO \\
\hline 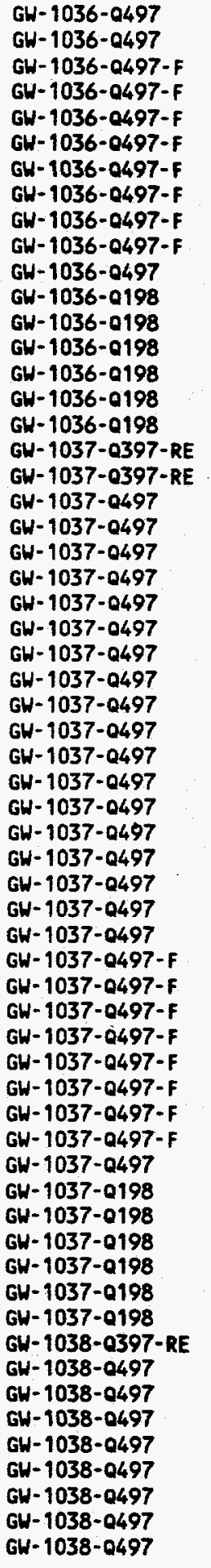 & $\begin{array}{l}10 / 21 / 97 \\
10 / 21 / 97 \\
10 / 21 / 97 \\
10 / 21 / 97 \\
10 / 21 / 97 \\
10 / 21 / 97 \\
10 / 21 / 97 \\
10 / 21 / 97 \\
10 / 21 / 97 \\
10 / 21 / 97 \\
10 / 21 / 97 \\
01 / 27 / 98 \\
01 / 27 / 98 \\
01 / 27 / 98 \\
01 / 27 / 98 \\
01 / 27 / 98 \\
01 / 27 / 98 \\
08 / 11 / 97 \\
08 / 11 / 97 \\
10 / 21 / 97 \\
10 / 21 / 97 \\
10 / 21 / 97 \\
10 / 21 / 97 \\
10 / 21 / 97 \\
10 / 21 / 97 \\
10 / 21 / 97 \\
10 / 21 / 97 \\
10 / 21 / 97 \\
10 / 21 / 97 \\
10 / 21 / 97 \\
10 / 21 / 97 \\
10 / 21 / 97 \\
10 / 21 / 97 \\
10 / 21 / 97 \\
10 / 21 / 97 \\
10 / 21 / 97 \\
10 / 21 / 97 \\
10 / 21 / 97 \\
10 / 21 / 97 \\
10 / 21 / 97 \\
10 / 21 / 97 \\
10 / 21 / 97 \\
10 / 21 / 97 \\
10 / 21 / 97 \\
10 / 21 / 97 \\
10 / 21 / 97 \\
01 / 27 / 98 \\
01 / 27 / 98 \\
01 / 27 / 98 \\
01 / 27 / 98 \\
01 / 27 / 98 \\
01 / 27 / 98 \\
08 / 11 / 97 \\
10 / 21 / 97 \\
10 / 21 / 97 \\
10 / 21 / 97 \\
10 / 21 / 97 \\
10 / 21 / 97 \\
10 / 21 / 97 \\
10 / 21 / 97 \\
10 / 21 / 97\end{array}$ & 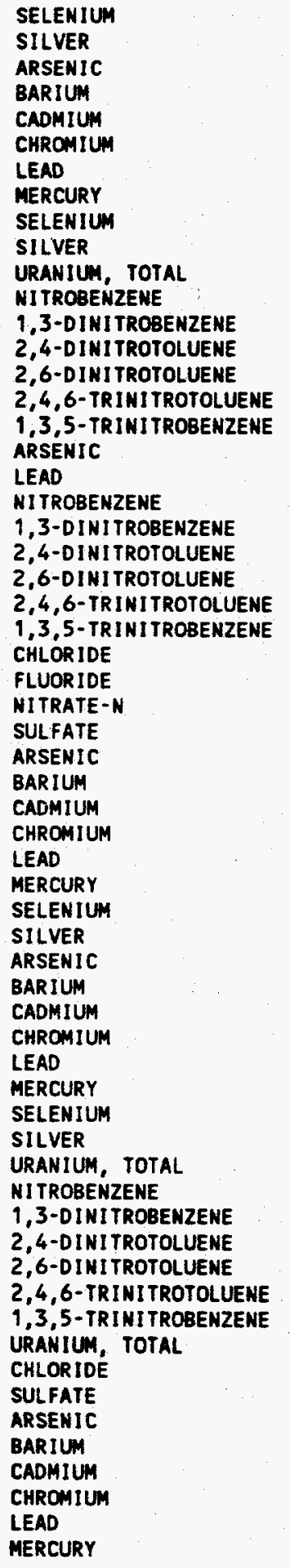 & 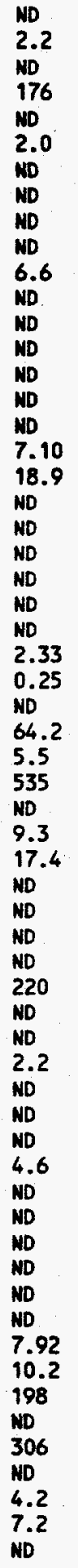 & $\begin{array}{l}\text { S.0 } \\
1.0 \\
4.0 \\
2.0 \\
2.0 \\
2.0 \\
2.0 \\
0.10 \\
5.0 \\
1.0 \\
0.2 \\
0.030 \\
0.090 \\
0.030 \\
0.010 \\
0.030 \\
0.030 \\
3.00 \\
2.00 \\
0.030 \\
0.090 \\
0.030 \\
0.010 \\
0.030 \\
0.030 \\
0.25 \\
0.05 \\
0.03 \\
5.0\end{array}$ & $\begin{array}{l}U G / L \\
U G / L \\
U G / L \\
U G / L \\
U G / L \\
U G / L \\
U G / L \\
U G / L \\
U G / L \\
U G / L \\
P C I / L \\
U G / L \\
U G / L \\
U G / L \\
U G / L \\
U G / L \\
U G / L \\
U G / L \\
U G / L \\
U G / L \\
U G / L \\
U G / L \\
U G / L \\
U G / L \\
U G / L \\
M G / L \\
M G / L \\
M G / L \\
M G / L \\
U G / L \\
U G / L \\
U G / L \\
U G / L \\
U G / L \\
U G / L \\
U G / L \\
U G / L \\
U G / L \\
U G / L \\
U G / L \\
U G / L \\
U G / L \\
U G / L \\
U G / L \\
U G / L \\
P C I / L \\
U G / L \\
U G / L \\
U G / L \\
U G / L \\
U G / L \\
U G / L \\
P C I / L \\
M G / L \\
M G / L \\
U G / L \\
U G / L \\
U G / L \\
U G / L \\
U G / L \\
U G / L \\
U\end{array}$ & $\begin{array}{l}\text { EPA } 6010 A \\
\text { EPA } 6010 A \\
\text { EPA } 6010 A \\
\text { EPA } 6010 A \\
\text { EPA } 6010 A \\
\text { EPA } 6010 A \\
\text { EPA } 6010 A \\
\text { EPA } 7470 A \\
\text { EPA } 6010 A \\
\text { EPA } 6010 A \\
\text { ASTM D2907 } \\
\text { USATHAMA } \\
\text { USATHAMA } \\
\text { USATHAMA } \\
\text { USATHAMA } \\
\text { USATHAMA } \\
\text { USATHAMA } \\
\text { EPA CLP } \\
\text { EPA CLP } \\
\text { USATHAMA } \\
\text { USATHAMA } \\
\text { USATHAMA } \\
\text { USATHAMA } \\
\text { USATHAMA } \\
\text { USATHAMA } \\
\text { EPA } 300.0 \\
\text { EPA } 340 \\
\text { EPA } 353.1 \\
\text { EPA } 300.0 \\
\text { EPA } 6010 A \\
\text { EPA } 6010 A \\
\text { EPA } 6010 A \\
\text { EPA } 6010 A \\
\text { EPA } 6010 A \\
\text { EPA } 7470 A \\
\text { EPA } 6010 A \\
\text { EPA } 6010 A \\
\text { EPA } 6010 A \\
\text { EPA } 6010 A \\
\text { EPA } 6010 A \\
\text { EPA } 6010 A \\
\text { EPA } 6010 A \\
\text { EPA } 7470 A \\
\text { EPA } 6010 A \\
\text { EPA } 6010 A \\
\text { ASTM D2907 } \\
\text { USATHAMA } \\
\text { USATHAMA } \\
\text { USATHAMA } \\
\text { USATHAMA } \\
\text { USATHAMA } \\
\text { USATHAMA } \\
\text { EPA } 6020 \\
\text { EPA } 300.0 \\
\text { EPA } 300.0 \\
\text { EPA } 6010 A \\
\text { EPA } 6010 A \\
\text { EPA } 6010 A \\
\text { EPA } 6010 A \\
\text { EPA } 6010 A \\
\text { EPA } 7470 A \\
\end{array}$ \\
\hline
\end{tabular}




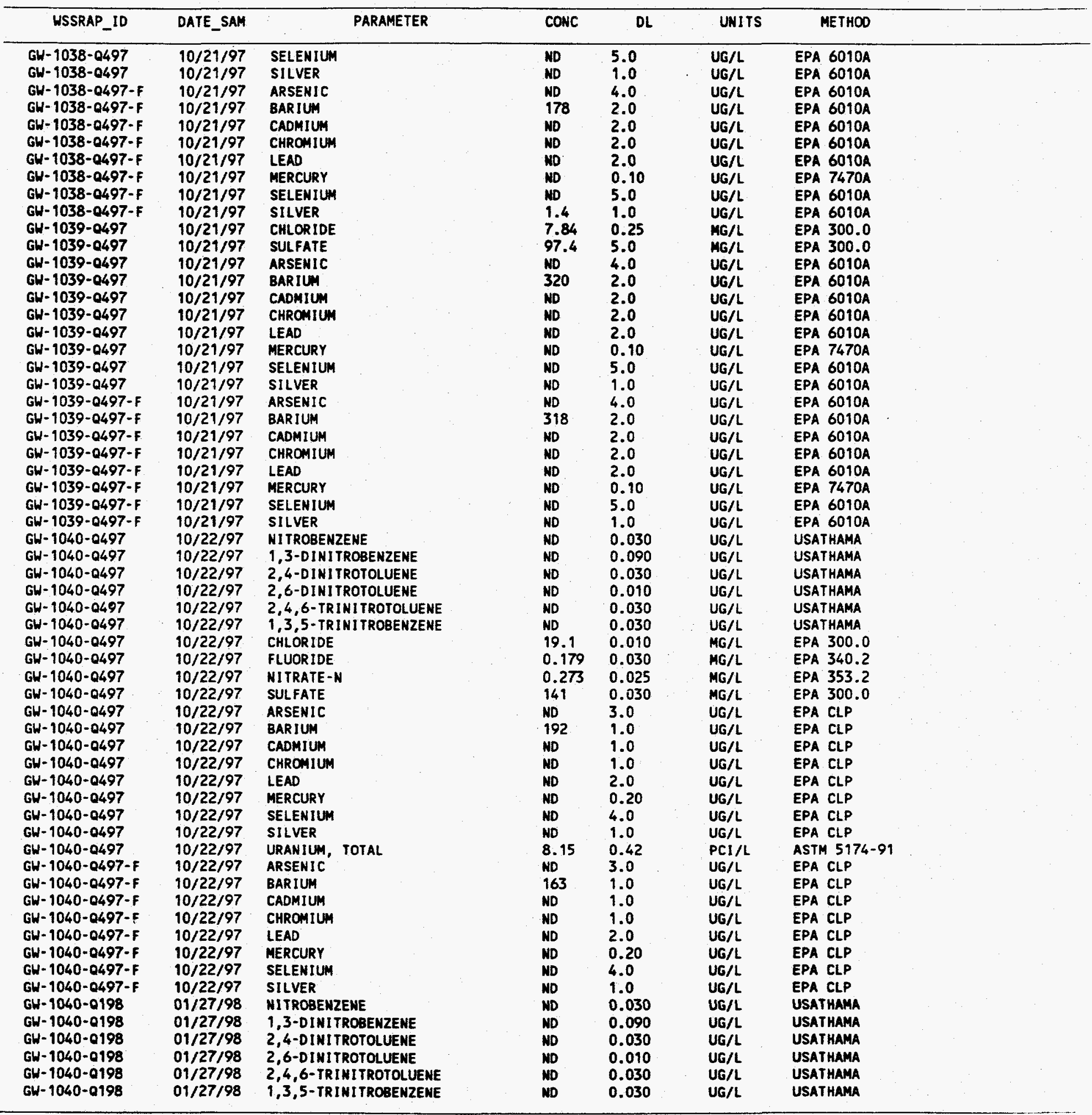




\begin{tabular}{|c|c|c|c|c|c|c|}
\hline WSSRAP_ID & DATE_SAM & PARAMETER & CONC & $D L$ & UNITS & METHOO \\
\hline $\begin{array}{l}\text { GW-1041-0497 } \\
G W-1041-0497 \\
G W-1041-0497 \\
G W-1041-0497 \\
G W-1041-0497 \\
G W-1041-0497 \\
G W-1041-0497 \\
G W-1041-0497 \\
G W-1041-0497 \\
G W-1041-0497 \\
G W-1041-0497 \\
G W-1041-0497 \\
G W-1041-0497 \\
G W-1041-0497 \\
G W-1041-0497 \\
G W-1041-0497 \\
G W-1041-0497 \\
G W-1041-0497 \\
G W-1041-0497 \\
G W-1041-0497-F \\
G W-1041-0497-F \\
G W-1041-0497-F \\
G W-1041-0497-F \\
G H-1041-0497-F \\
G W-1041-0497-F \\
G W-1041-0497-F \\
G W-1041-0497-F \\
G W-1041-0198 \\
G W-1041-0198 \\
G W-1041-0198 \\
G W-1041-0198 \\
G W-1041-0198 \\
G W-1041-0198 \\
G W-2013-1297 \\
G W-2013-1297 \\
G W-2013-1297 \\
G W-2013-1297 \\
G W-2013-1297 \\
G W-2013-1297 \\
G W-2013-1297 \\
G W-2013-1297 \\
G W-2013-1297 \\
G W-2013-1297 \\
G W-2013-1297 \\
G W-2013-1297 \\
G W-2013-1297 \\
G W-2013-1297 \\
G W-2013-1297 \\
G W-2013-1297 \\
G W-2013-1297 \\
G W-2013-1297 \\
G W-2013-1297 \\
G W-2013-1297 \\
G W-2013-1297 \\
G W-2013-1297 \\
G W-2013-1297 \\
G W-2013-1297 \\
G W-2013-1297 \\
G W-2013-1297 \\
G W-2013-1297 \\
G W-2013-1297\end{array}$ & $\begin{array}{l}10 / 22 / 97 \\
10 / 22 / 97 \\
10 / 22 / 97 \\
10 / 22 / 97 \\
10 / 22 / 97 \\
10 / 22 / 97 \\
10 / 22 / 97 \\
10 / 22 / 97 \\
10 / 22 / 97 \\
10 / 22 / 97 \\
10 / 22 / 97 \\
10 / 22 / 97 \\
10 / 22 / 97 \\
10 / 22 / 97 \\
10 / 22 / 97 \\
10 / 22 / 97 \\
10 / 22 / 97 \\
10 / 22 / 97 \\
10 / 22 / 97 \\
10 / 22 / 97 \\
10 / 22 / 97 \\
10 / 22 / 97 \\
10 / 22 / 97 \\
10 / 22 / 97 \\
10 / 22 / 97 \\
10 / 22 / 97 \\
10 / 22 / 97 \\
01 / 27 / 98 \\
01 / 27 / 98 \\
01 / 27 / 98 \\
01 / 27 / 98 \\
01 / 27 / 98 \\
01 / 27 / 98 \\
12 / 01 / 97 \\
12 / 01 / 97 \\
12 / 01 / 97 \\
12 / 01 / 97 \\
12 / 01 / 97 \\
12 / 01 / 97 \\
12 / 01 / 97 \\
12 / 01 / 97 \\
12 / 01 / 97 \\
12 / 01 / 97 \\
12 / 01 / 97 \\
12 / 01 / 97 \\
12 / 01 / 97 \\
12 / 01 / 97 \\
12 / 01 / 97 \\
12 / 01 / 97 \\
12 / 01 / 97 \\
12 / 01 / 97 \\
12 / 01 / 97 \\
12 / 01 / 97 \\
12 / 01 / 97 \\
12 / 01 / 97 \\
12 / 01 / 97 \\
12 / 01 / 97 \\
12 / 01 / 97 \\
12 / 01 / 97 \\
12 / 01 / 97 \\
12 / 01 / 97\end{array}$ & 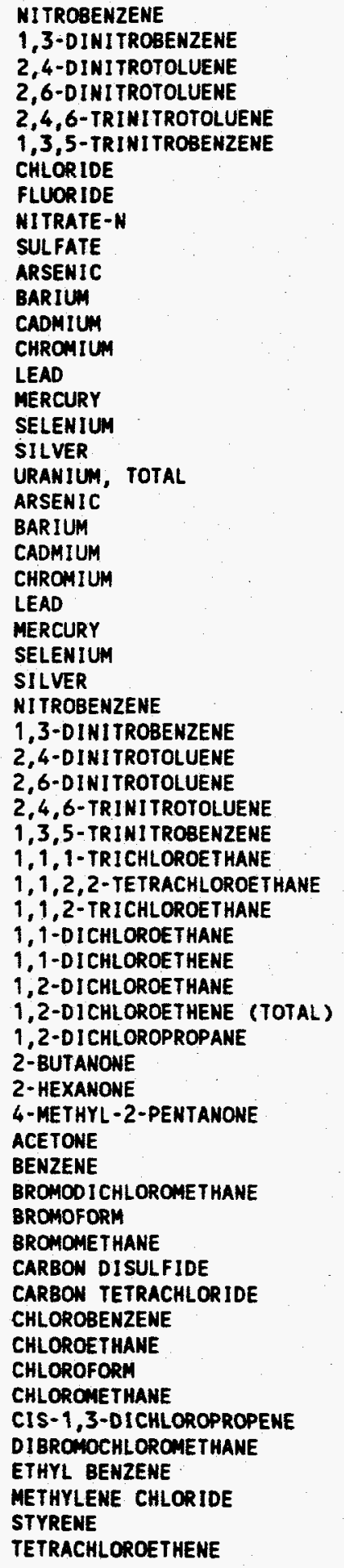 & $\begin{array}{l}\text { ND } \\
\text { ND } \\
\text { ND } \\
\text { ND } \\
\text { ND } \\
\text { ND } \\
5.48 \\
0.178 \\
0.238 \\
47.4 \\
\text { ND } \\
\text { 377 } \\
\text { ND } \\
\text { ND } \\
\text { ND } \\
\text { ND } \\
\text { ND } \\
\text { ND } \\
\text { 5.54 } \\
\text { ND } \\
\text { 385 } \\
\text { ND } \\
\text { ND } \\
\text { ND } \\
\text { ND } \\
\text { ND } \\
\text { ND. } \\
\text { ND } \\
\text { ND } \\
\text { ND } \\
\text { ND } \\
\text { ND } \\
\text { ND } \\
\text { ND } \\
\text { ND } \\
\text { ND } \\
\text { ND } \\
\text { ND } \\
\text { ND } \\
\text { C6) } \\
\text { ND } \\
\text { ND } \\
\text { ND } \\
\text { ND } \\
\text { ND } \\
\text { ND } \\
\text { ND } \\
\text { ND } \\
\text { ND } \\
\text { ND } \\
\text { ND } \\
\text { ND } \\
\text { ND } \\
\text { ND } \\
\text { ND } \\
\text { ND } \\
\text { ND } \\
\text { ND } \\
\text { ND } \\
\text { ND } \\
\text { ND }\end{array}$ & $\begin{array}{l}0.030 \\
0.090 \\
0.030 \\
0.010 \\
0.030 \\
0.030 \\
0.010 \\
0.030 \\
0.025 \\
0.030 \\
3.0 \\
1.0 \\
1.0 \\
1.0 \\
2.0 \\
0.20 \\
4.0 \\
1.0 \\
0.42 \\
3.0 \\
1.0 \\
1.0 \\
1.0 \\
2.0 \\
0.20 \\
4.0 \\
1.0 \\
0.030 \\
0.090 \\
0.030 \\
0.010 \\
0.030 \\
0.030 \\
10 \\
10 \\
10 \\
10 \\
10 \\
10 \\
10 \\
10 \\
10 \\
10 \\
10 \\
10 \\
10 \\
10 \\
10 \\
10 \\
10 \\
10 \\
10 \\
10 \\
10 \\
10 \\
10 \\
10 \\
10 \\
10 \\
10 \\
10 \\
10 \\
0\end{array}$ & 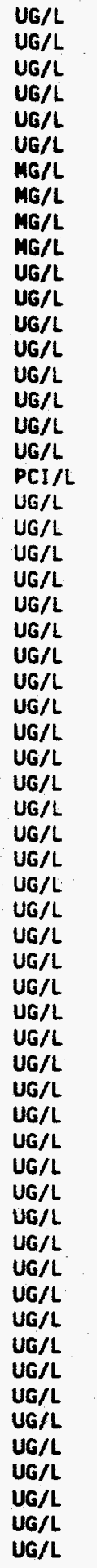 & $\begin{array}{l}\text { USATHAMA } \\
\text { USATHAMA } \\
\text { USATHAMA } \\
\text { USATHAMA } \\
\text { USATHAMA } \\
\text { USATHAMA } \\
\text { EPA } 300.0 \\
\text { EPA } 340.2 \\
\text { EPA } 353.2 \\
\text { EPA } 300.0 \\
\text { EPA CLP } \\
\text { EPA CLP } \\
\text { EPA CLP } \\
\text { EPA CLP } \\
\text { EPA CLP } \\
\text { EPA CLP } \\
\text { EPA CLP } \\
\text { EPA CLP } \\
\text { ASTM } 5174-91 \\
\text { EPA CLP } \\
\text { EPA CLP } \\
\text { EPA CLP } \\
\text { EPA CLP } \\
\text { EPA CLP } \\
\text { EPA CLP } \\
\text { EPA CLP } \\
\text { EPA CLP } \\
\text { USATHAMA } \\
\text { USATHAMA } \\
\text { USATHAMA } \\
\text { USATHAMA } \\
\text { USATHAMA } \\
\text { USATHAMA } \\
\text { EPA CLP } \\
\text { EPA CLP } \\
\text { EPA CLP } \\
\text { EPA CLP } \\
\text { EPA CLP } \\
\text { EPA CLP } \\
\text { EPA CLP } \\
\text { EPA CLP } \\
\text { EPA CLP } \\
\text { EPA CLP } \\
\text { EPA CLP } \\
\text { EPA CLP } \\
\text { EPA CLP } \\
\text { EPA CLP } \\
\text { EPA CLP } \\
\text { EPA CLP } \\
\text { EPA CLP } \\
\text { EPA CLP } \\
\text { EPA CLP } \\
\text { EPA CLP } \\
\text { EPA CLP } \\
\text { EPA CLP } \\
\text { EPA CLP } \\
\text { EPA CLP } \\
\text { EPA CLP } \\
\text { EPA CLP } \\
\text { EPA CLP } \\
\text { EPA CLP } \\
\end{array}$ \\
\hline
\end{tabular}




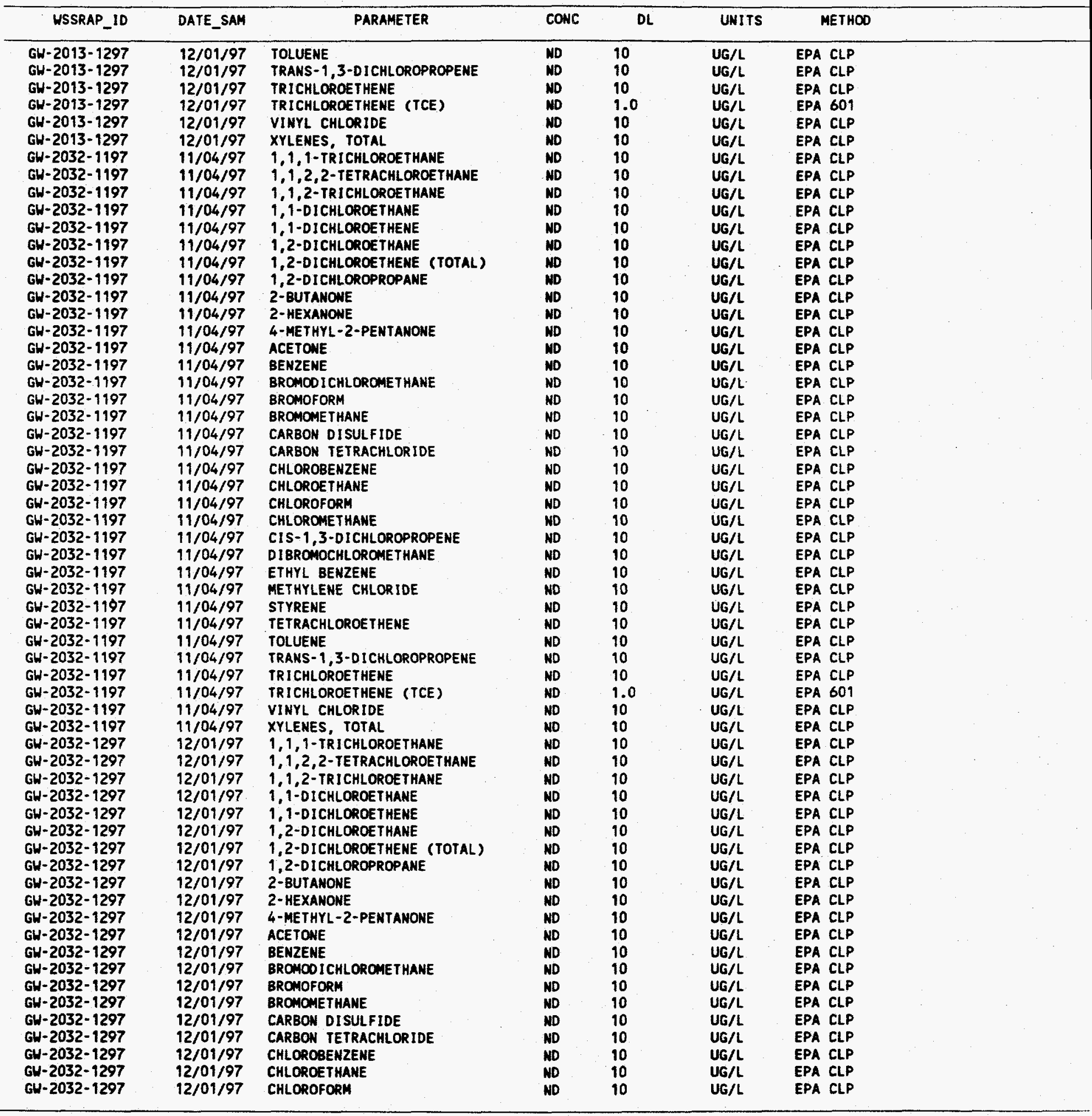




\begin{tabular}{|c|c|c|c|c|c|c|}
\hline WSSRAP_ID & OATE_SAM & PARAMETER & CONC & DL & UNITS & METHOO \\
\hline 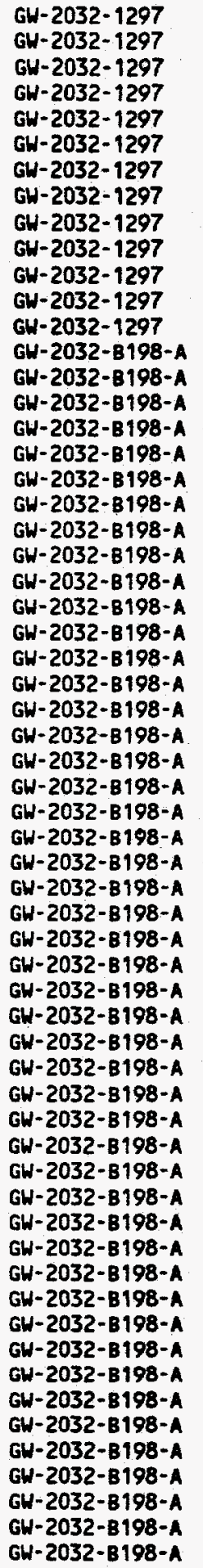 & $\begin{array}{l}12 / 01 / 97 \\
12 / 01 / 97 \\
12 / 01 / 97 \\
12 / 01 / 97 \\
12 / 01 / 97 \\
12 / 01 / 97 \\
12 / 01 / 97 \\
12 / 01 / 97 \\
12 / 01 / 97 \\
12 / 01 / 97 \\
12 / 01 / 97 \\
12 / 01 / 97 \\
12 / 01 / 97 \\
01 / 19 / 98 \\
01 / 19 / 98 \\
01 / 19 / 98 \\
01 / 19 / 98 \\
01 / 19 / 98 \\
01 / 19 / 98 \\
01 / 19 / 98 \\
01 / 19 / 98 \\
01 / 19 / 98 \\
01 / 19 / 98 \\
01 / 19 / 98 \\
01 / 19 / 98 \\
01 / 19 / 98 \\
01 / 19 / 98 \\
01119 / 98 \\
01 / 19 / 98 \\
01 / 19 / 98 \\
01 / 19 / 98 \\
01 / 19 / 98 \\
011 / 19 / 98 \\
011 / 19 / 98 \\
01 / 19 / 98 \\
01 / 19 / 98 \\
01 / 19 / 98 \\
011 / 19 / 98 \\
01119 / 98 \\
01 / 19 / 98 \\
01 / 19 / 98 \\
011119 / 98 \\
011 / 19 / 98 \\
01 / 19 / 98 \\
01 / 19 / 98 \\
01119 / 98 \\
011 / 19 / 98 \\
01 / 19 / 98 \\
01 / 19 / 98 \\
01 / 19 / 98 \\
01119 / 98 \\
01119 / 98 \\
01 / 19 / 98 \\
01 / 19 / 98 \\
01 / 19 / 98 \\
01 / 19 / 98 \\
011 / 19 / 98 \\
01 / 19 / 98 \\
01 / 19 / 98 \\
01 / 19 / 98 \\
01 / 19 / 98\end{array}$ & 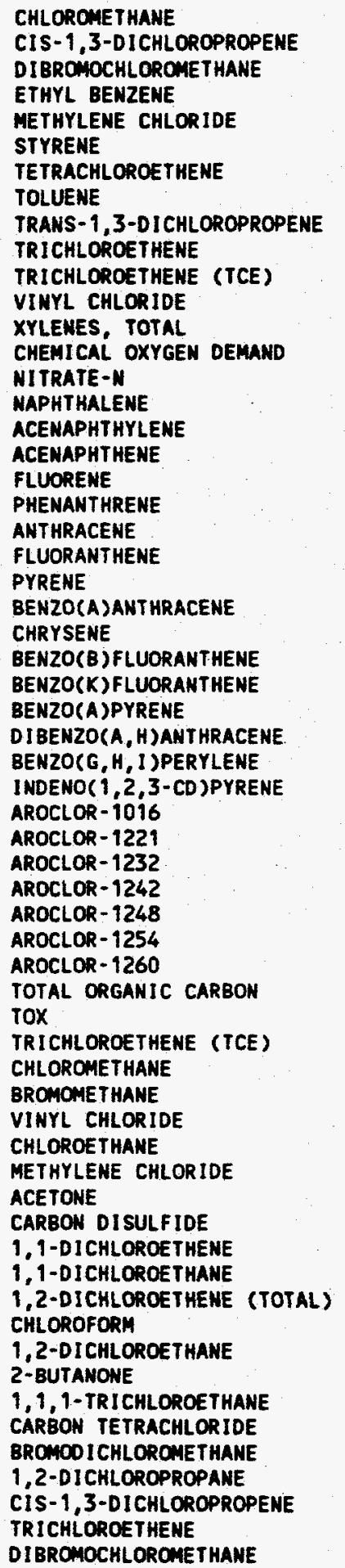 & 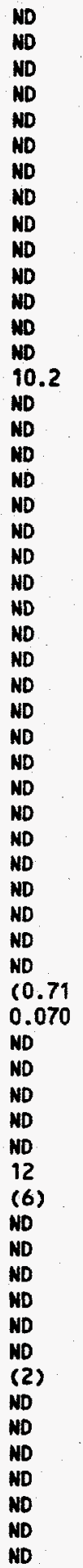 & $\begin{array}{l}10 \\
10 \\
10 \\
10 \\
10 \\
10 \\
10 \\
10 \\
10 \\
10 \\
1.0 \\
10 \\
10 \\
5.00 \\
1.00 \\
5.0 \\
5.0 \\
5.0 \\
5.0 \\
5.0 \\
5.0 \\
5.0 \\
5.0 \\
5\end{array}$ & 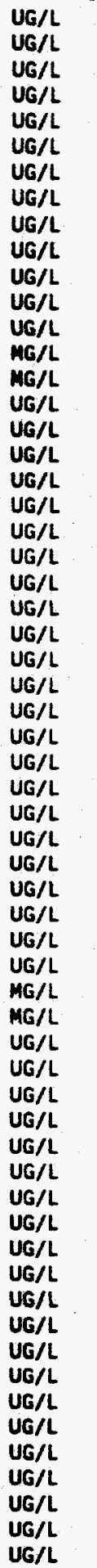 & 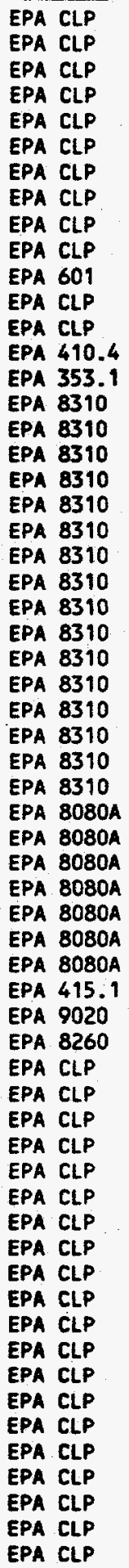 \\
\hline
\end{tabular}




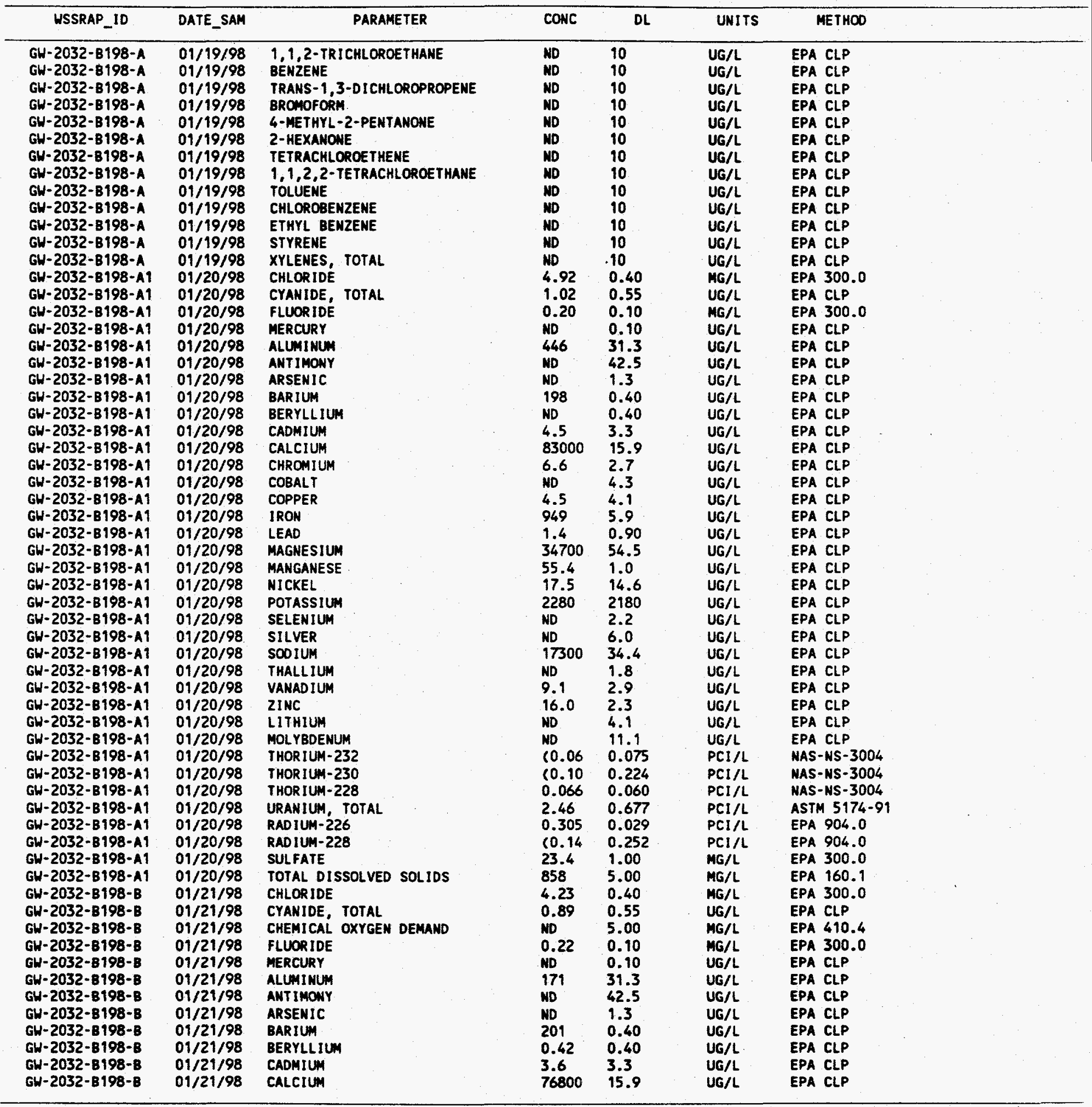




\begin{tabular}{|c|c|c|c|c|c|c|}
\hline WSSRAP_ID & OATE_SAM & PARAMETER & CONC & OL & UNITS & METHOO \\
\hline 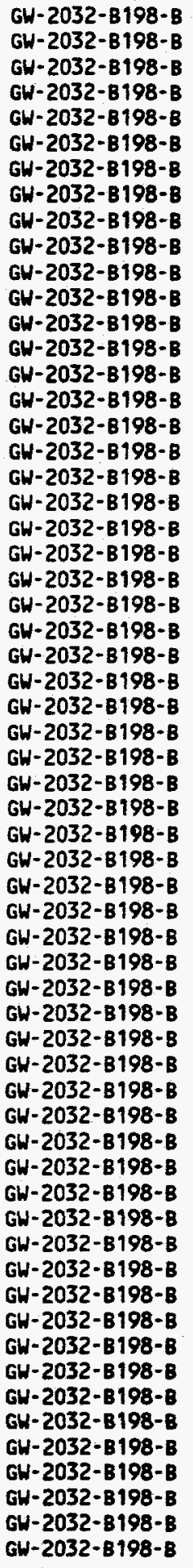 & $\begin{array}{l}01 / 21 / 98 \\
01 / 21 / 98 \\
01 / 21 / 98 \\
01 / 21 / 98 \\
01 / 21 / 98 \\
01 / 21 / 98 \\
01 / 21 / 98 \\
01 / 21 / 98 \\
01 / 21 / 98 \\
01 / 21 / 98 \\
01 / 21 / 98 \\
01 / 21 / 98 \\
01 / 21 / 98 \\
01 / 21 / 98 \\
01 / 21 / 98 \\
01 / 21 / 98 \\
01 / 21 / 98 \\
01 / 21 / 98 \\
01 / 21 / 98 \\
01 / 21 / 98 \\
01 / 21 / 98 \\
01 / 21 / 98 \\
01 / 21 / 98 \\
01 / 21 / 98 \\
01 / 21 / 98 \\
01 / 21 / 98 \\
01 / 21 / 98 \\
01 / 21 / 98 \\
01 / 21 / 98 \\
01 / 21 / 98 \\
01 / 21 / 98 \\
01 / 21 / 98 \\
01 / 21 / 98 \\
01 / 21 / 98 \\
01 / 21 / 98 \\
01 / 21 / 98 \\
01 / 21 / 98 \\
01 / 21 / 98 \\
01 / 21 / 98 \\
01 / 21 / 98 \\
01 / 21 / 98 \\
01 / 21 / 98 \\
01 / 21 / 98 \\
01 / 21 / 98 \\
01 / 21 / 98 \\
01 / 21 / 98 \\
01 / 21 / 98 \\
01 / 21 / 98 \\
01 / 21 / 98 \\
01 / 21 / 98 \\
01 / 21 / 98 \\
01 / 21 / 98 \\
01 / 21 / 98 \\
01 / 21 / 98 \\
01 / 21 / 98 \\
01 / 21 / 98 \\
01 / 21 / 98 \\
01 / 21 / 98 \\
01 / 21 / 98 \\
01 / 21 / 98 \\
01 / 21 / 98\end{array}$ & 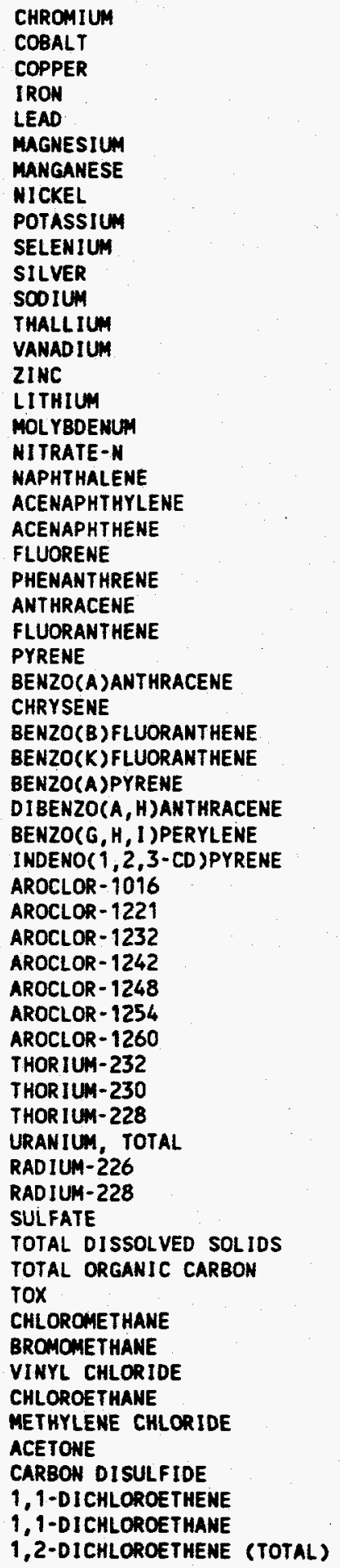 & $\begin{array}{l}\text { ND } \\
\text { ND } \\
10.6 \\
279 \\
\text { ND } \\
33700 \\
20.6 \\
19.0 \\
\text { ND } \\
\text { ND } \\
\text { ND } \\
\text { 15600 } \\
\text { ND } \\
8.7 \\
10.1 \\
\text { ND } \\
13.6 \\
15.8 \\
\text { ND } \\
\text { ND } \\
\text { ND } \\
\text { ND } \\
\text { ND } \\
\text { ND } \\
\text { ND } \\
\text { ND } \\
\text { ND } \\
\text { ND } \\
\text { ND } \\
\text { ND } \\
\text { ND } \\
\text { ND } \\
\text { ND } \\
\text { ND } \\
\text { ND } \\
\text { ND } \\
\text { ND } \\
\text { ND } \\
\text { ND } \\
\text { ND } \\
\text { ND } \\
\text { C0.02 } \\
0.184 \\
0.077 \\
2.29 \\
0.273 \\
\text { C0.16 } \\
29.0 \\
\text { 409 } \\
\text { ND } \\
0.020 \\
\text { ND } \\
\text { ND } \\
\text { ND } \\
\text { ND } \\
\text { SO } \\
\text { C5) } \\
\text { ND } \\
\text { ND } \\
\text { ND } \\
\text { ND }\end{array}$ & 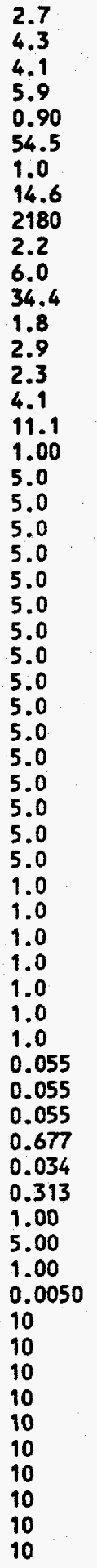 & 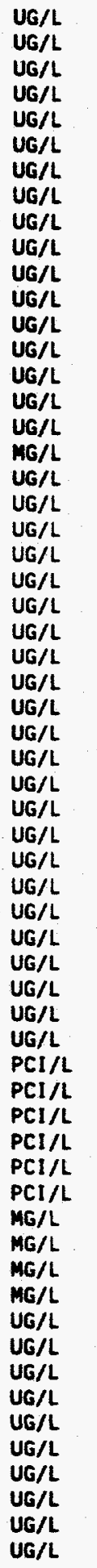 & $\begin{array}{l}\text { EPA CLP } \\
\text { EPA CLP } \\
\text { EPA CLP } \\
\text { EPA CLP } \\
\text { EPA CLP } \\
\text { EPA CLP } \\
\text { EPA CLP } \\
\text { EPA CLP } \\
\text { EPA CLP } \\
\text { EPA CLP } \\
\text { EPA CLP } \\
\text { EPA CLP } \\
\text { EPA CLP } \\
\text { EPA CLP } \\
\text { EPA CLP } \\
\text { EPA CLP } \\
\text { EPA CLP } \\
\text { EPA } 353.1 \\
\text { EPA } 8310 \\
\text { EPA } 8310 \\
\text { EPA } 8310 \\
\text { EPA } 8310 \\
\text { EPA } 8310 \\
\text { EPA } 8310 \\
\text { EPA } 8310 \\
\text { EPA } 8310 \\
\text { EPA } 8310 \\
\text { EPA } 8310 \\
\text { EPA } 8310 \\
\text { EPA } 8310 \\
\text { EPA } 8310 \\
\text { EPA } 8310 \\
\text { EPA } 8310 \\
\text { EPA } 8310 \\
\text { EPA } 8080 A \\
\text { EPA } 8080 A \\
\text { EPA } 8080 A \\
\text { EPA } 8080 A \\
\text { EPA } 8080 A \\
\text { EPA } 8080 A \\
\text { EPA } 8080 A \\
\text { NAS-NS-3004 } \\
\text { NAS-NS-3004 } \\
\text { NAS- }-35-3004 \\
\text { ASTM } 5174-91 \\
\text { EPA } 904.0 \\
\text { EPA } 904.0 \\
\text { EPA } 300.0 \\
\text { EPA } 160.1 \\
\text { EPA } 415.1 \\
\text { EPA } 9020 \\
\text { EPA CLP } \\
\text { EPA CLP } \\
\text { EPA CLP } \\
\text { EPA CLP } \\
\text { EPA CLP } \\
\text { EPA CLP } \\
\text { EPA CLP } \\
\text { EPA CLP } \\
\text { EPA CLP } \\
\text { EPA CLP } \\
\text { EPA }\end{array}$ \\
\hline
\end{tabular}




\begin{tabular}{|c|c|c|c|c|c|c|}
\hline WSSRAP_ID & DATE_SAM & PARAMETER & COHC & $D L$ & UNITS & METHOD \\
\hline $\begin{array}{l}G W-2032-B 198-B \\
G W-2032-B 198-B \\
G W-2032-B 198-B \\
G W-2032-B 198-B \\
G W-2032-B 198-B \\
G W-2032-B 198-B \\
G W-2032-B 198-B \\
G W-2032-8198-B \\
G W-2032-8198-B \\
G W-2032-8198-B \\
G W-2032-B 198-B \\
G W-2032-B 198-B \\
G W-2032-B 198-B \\
G W-2032-B 198-B \\
G W-2032-B 198-B \\
G W-2032-B 198-B \\
G W-2032-B 198-B \\
G W-2032-B 198-B \\
G W-2032-8198-B \\
G W-2032-B 198-B \\
G W-2032-B 198-B \\
G W-2032-B 198-B \\
G W-2032-B 198-B \\
G W-2032-B 198-C \\
G W-2032-B 198-C \\
G W-2032-B 198-C \\
G W-2032-B 198-C \\
G W-2032-B 198-C \\
G W-2032-B 198-C \\
G W-2032-B 198-C \\
G W-2032-B 198-C \\
G W-2032-B 198-C \\
G W-2032-B 198-C \\
G W-2032-B 198-C \\
G W-2032-B 198-C \\
G W-2032-B 198-C \\
G W-2032-B 198-C \\
G W-2032-8198-C \\
G W-2032-8198-C \\
G W-2032-B 198-C \\
G W-2032-8198-C \\
G W-2032-B 198-C \\
G W-2032-B 198-C \\
G W-2032-B 198-C \\
G W-2032-B 198-C \\
G W-2032-B 198-C \\
G W-2032-B 198-C \\
G W-2032-B 198-C \\
G W-2032-B 198-C \\
G W-2032-B 198-C \\
G W-2032-B 198-C \\
G W-2032-B 198-C \\
G W-2032-B 198-C \\
G W-2032-B 198-C \\
G W-2032-B 198-C \\
G W-2032-B 198-C \\
G W-2032-B 198-C \\
G W-2032-B 198-C \\
G W-2032-B 198-C \\
G W-2032-B 198-C \\
G W-2032-B 198-C\end{array}$ & $\begin{array}{l}01 / 21 / 98 \\
01 / 21 / 98 \\
01 / 21 / 98 \\
01 / 21 / 98 \\
01 / 21 / 98 \\
01 / 21 / 98 \\
01 / 21 / 98 \\
01 / 21 / 98 \\
01 / 21 / 98 \\
01 / 21 / 98 \\
01 / 21 / 98 \\
01 / 21 / 98 \\
01 / 21 / 98 \\
01 / 21 / 98 \\
01 / 21 / 98 \\
01 / 21 / 98 \\
01 / 21 / 98 \\
01 / 21 / 98 \\
01 / 21 / 98 \\
01 / 21 / 98 \\
01 / 21 / 98 \\
01 / 21 / 98 \\
01 / 21 / 98 \\
01 / 22 / 98 \\
01 / 22 / 98 \\
01 / 22 / 98 \\
01 / 22 / 98 \\
01 / 22 / 98 \\
01 / 22 / 98 \\
01 / 22 / 98 \\
01 / 22 / 98 \\
01 / 22 / 98 \\
01 / 22 / 98 \\
01 / 22 / 98 \\
01 / 22 / 98 \\
011 / 22 / 98 \\
01 / 22 / 98 \\
01 / 22 / 98 \\
01 / 22 / 98 \\
011 / 22 / 98 \\
01 / 22 / 98 \\
01 / 22 / 98 \\
01 / 22 / 98 \\
011 / 22 / 98 \\
01 / 22 / 98 \\
01 / 22 / 98 \\
01 / 22 / 98 \\
01 / 22 / 98 \\
011 / 22 / 98 \\
01 / 22 / 98 \\
01 / 22 / 98 \\
01 / 22 / 98 \\
01 / 22 / 98 \\
011 / 22 / 98 \\
01 / 22 / 98 \\
01 / 22 / 98 \\
01 / 22 / 98 \\
01 / 22 / 98 \\
011 / 22 / 98 \\
01 / 22 / 98 \\
01 / 22 / 98\end{array}$ & 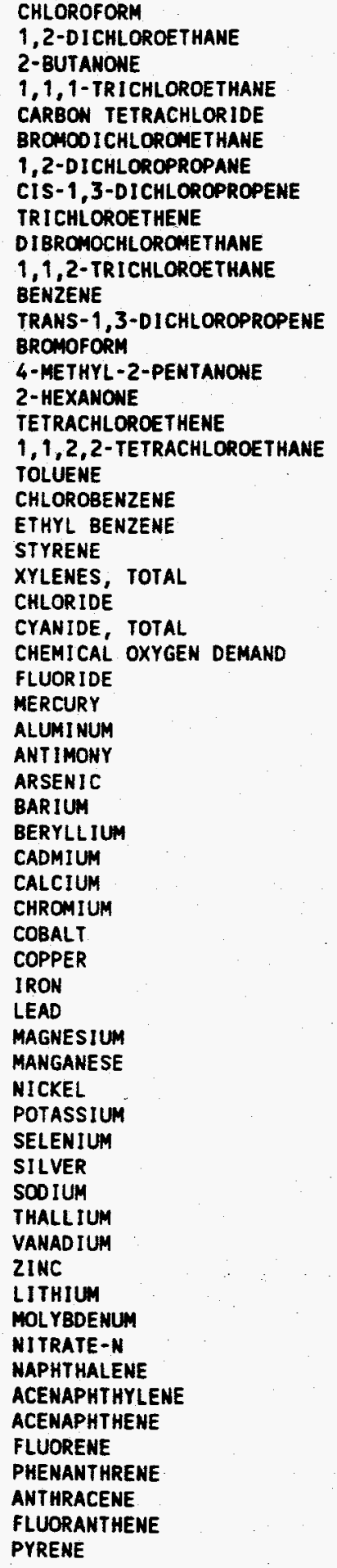 & $\begin{array}{l}\text { ND } \\
\text { ND } \\
\text { ND } \\
\text { ND } \\
\text { ND } \\
\text { ND } \\
\text { ND } \\
\text { ND } \\
\text { ND } \\
\text { ND } \\
\text { ND } \\
\text { ND } \\
\text { ND } \\
\text { ND } \\
\text { ND } \\
\text { ND } \\
\text { ND } \\
\text { ND } \\
\text { ND } \\
\text { ND } \\
\text { ND } \\
\text { ND } \\
\text { ND } \\
5.30 \\
0.60 \\
\text { ND } \\
0.23 \\
\text { ND } \\
295 \\
\text { ND } \\
1.6 \\
180 \\
1.9 \\
\text { ND } \\
77000 \\
7.2 \\
\text { ND } \\
13.4 \\
365 \\
\text { ND } \\
32400 \\
23.1 \\
16.5 \\
\text { ND } \\
4.4 \\
\text { ND } \\
19000 \\
5.0 \\
6.9 \\
\text { ND } \\
\text { ND } \\
\text { ND } \\
\text { ND } \\
\text { ND } \\
\text { ND } \\
\text { ND } \\
\text { ND } \\
\text { ND } \\
\text { ND } \\
\text { ND }\end{array}$ & $\begin{array}{l}10 \\
10 \\
10 \\
10 \\
10 \\
10 \\
10 \\
10 \\
10 \\
10 \\
10 \\
10 \\
10 \\
10 \\
10 \\
10 \\
10 \\
10 \\
10 \\
10 \\
10 \\
10 \\
10 \\
0.40 \\
0.55 \\
5.00 \\
0.10 \\
0.10 \\
31.3 \\
42.5 \\
1.3 \\
0.40 \\
0.40 \\
3.3 \\
15.9 \\
2.7 \\
4.3 \\
4.1 \\
5.9 \\
0.90 \\
54.5 \\
1.0 \\
14.6 \\
2180 \\
2.2 \\
6.0 \\
34.4 \\
1.8 \\
2.9 \\
2.3 \\
4.1 \\
11.1 \\
1.00 \\
5.0 \\
5.0 \\
5.0 \\
5.0 \\
5.0 \\
5\end{array}$ & 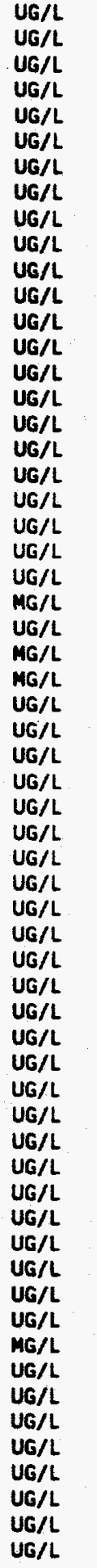 & $\begin{array}{l}\text { EPA CLP } \\
\text { EPA CLP } \\
\text { EPA CLP } \\
\text { EPA CLP } \\
\text { EPA CLP } \\
\text { EPA CLP } \\
\text { EPA CLP } \\
\text { EPA CLL } \\
\text { EPA CLP } \\
\text { EPA CLP } \\
\text { EPA CLP } \\
\text { EPA CLP } \\
\text { EPA CLP } \\
\text { EPA CLP } \\
\text { EPA CLP } \\
\text { EPA CLP } \\
\text { EPA CLP } \\
\text { EPA CLP } \\
\text { EPA CLP } \\
\text { EPA CLP } \\
\text { EPA CLP } \\
\text { EPA CLP } \\
\text { EPA CLP } \\
\text { EPA } 300.0 \\
\text { EPA CLP } \\
\text { EPA } 410.4 \\
\text { EPA } 300.0 \\
\text { EPA CLP } \\
\text { EPA CLP } \\
\text { EPA CLP } \\
\text { EPA CLP } \\
\text { EPA CLP } \\
\text { EPA CLP } \\
\text { EPA CLP } \\
\text { EPA CLP } \\
\text { EPA CLP } \\
\text { EPA CLP } \\
\text { EPA CLP } \\
\text { EPA CLP } \\
\text { EPA CLP } \\
\text { EPA CLP } \\
\text { EPA CLP } \\
\text { EPA CLP } \\
\text { EPA CLP } \\
\text { EPA CLP } \\
\text { EPA CLP } \\
\text { EPA CLP } \\
\text { EPA CLP } \\
\text { EPA CLP } \\
\text { EPA CLP } \\
\text { EPA CLP } \\
\text { EPA CLP } \\
\text { EPA } 353.1 \\
\text { EPA } 8310 \\
\text { EPA } 8310 \\
\text { EPA } 8310 \\
\text { EPA } 8310 \\
\text { EPA } 8310 \\
\text { EPA } 8310 \\
\text { EPA } 8310 \\
\text { EPA } 8310\end{array}$ \\
\hline
\end{tabular}




\begin{tabular}{|c|c|c|c|c|c|c|}
\hline WSSRAP_ID & DATE_SAM & PARAMETER & CONC & DL & UNITS & METHOO \\
\hline 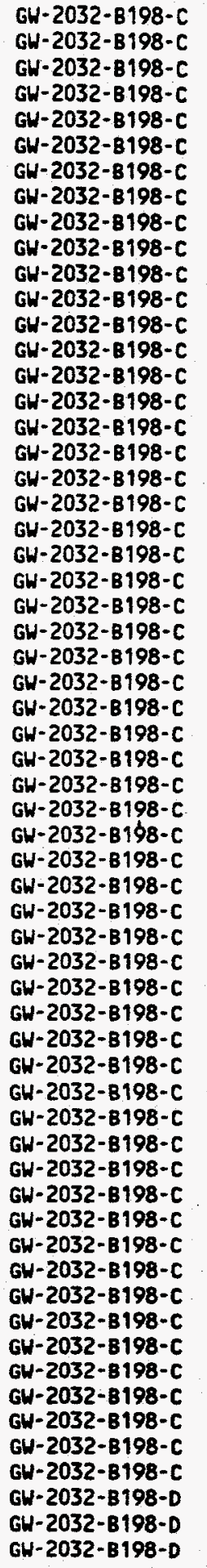 & $\begin{array}{l}01 / 22 / 98 \\
01 / 22 / 98 \\
01 / 22 / 98 \\
01 / 22 / 98 \\
01 / 22 / 98 \\
01 / 22 / 98 \\
01 / 22 / 98 \\
01 / 22 / 98 \\
01 / 22 / 98 \\
01 / 22 / 98 \\
01 / 22 / 98 \\
01 / 22 / 98 \\
01 / 22 / 98 \\
01 / 22 / 98 \\
01 / 22 / 98 \\
01 / 22 / 98 \\
01 / 22 / 98 \\
01 / 22 / 98 \\
01 / 22 / 98 \\
01 / 22 / 98 \\
01 / 22 / 98 \\
01 / 22 / 98 \\
01 / 22 / 98 \\
01 / 22 / 98 \\
01 / 22 / 98 \\
01 / 22 / 98 \\
01 / 22 / 98 \\
01 / 22 / 98 \\
011 / 22 / 98 \\
01 / 22 / 98 \\
01 / 22 / 98 \\
01 / 22 / 98 \\
01 / 22 / 98 \\
01 / 22 / 98 \\
01 / 22 / 98 \\
01 / 22 / 98 \\
01 / 22 / 98 \\
01 / 22 / 98 \\
01 / 22 / 98 \\
01 / 22 / 98 \\
01 / 22 / 98 \\
01 / 22 / 98 \\
01 / 22 / 98 \\
011 / 22 / 98 \\
01 / 22 / 98 \\
01 / 22 / 98 \\
01 / 22 / 98 \\
01 / 22 / 98 \\
011 / 22 / 98 \\
01 / 22 / 98 \\
01 / 22 / 98 \\
01 / 22 / 98 \\
01 / 22 / 98 \\
01 / 22 / 98 \\
01 / 22 / 98 \\
01 / 22 / 98 \\
01 / 22 / 98 \\
01 / 22 / 98 \\
01 / 23 / 98 \\
01 / 23 / 98 \\
01 / 23 / 98\end{array}$ & 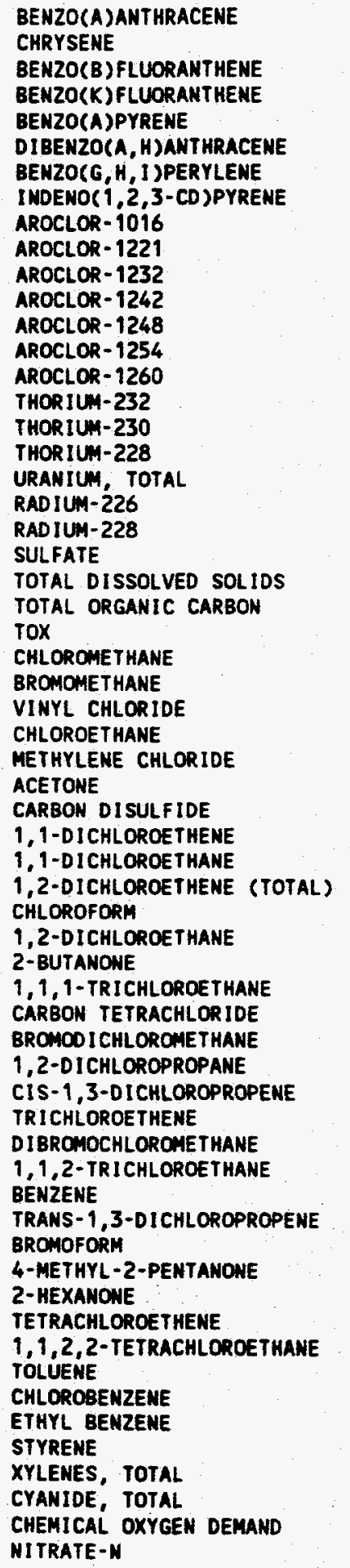 & 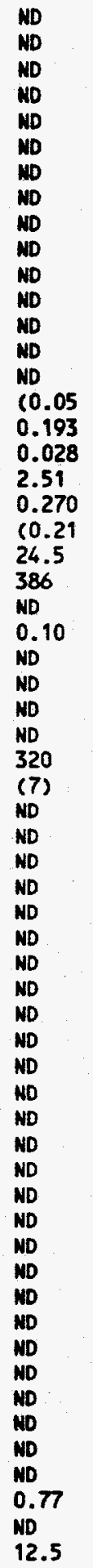 & 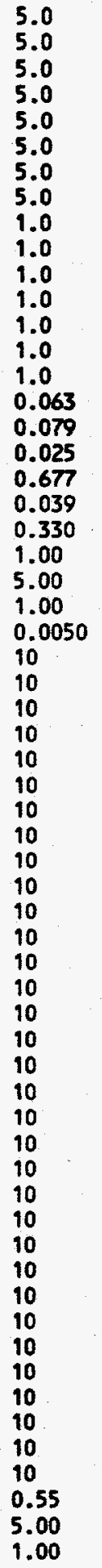 & 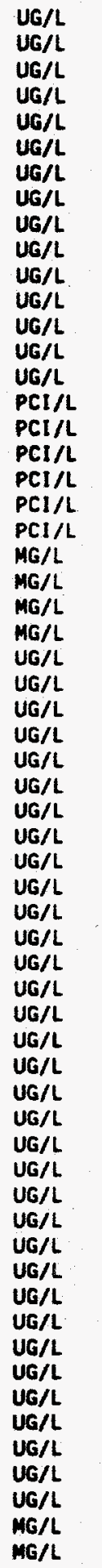 & $\begin{array}{l}\text { EPA } 8310 \\
\text { EPA } 8310 \\
\text { EPA } 8310 \\
\text { EPA } 8310 \\
\text { EPA } 8310 \\
\text { EPA } 8310 \\
\text { EPA } 8310 \\
\text { EPA } 8310 \\
\text { EPA } 8080 A \\
\text { EPA } 8080 A \\
\text { EPA } 8080 A \\
\text { EPA } 8080 A \\
\text { EPA } 8080 A \\
\text { EPA } 8080 A \\
\text { EPA } 8080 A \\
\text { NAS-NS-3004 } \\
\text { WAS-NS-3004 } \\
\text { MAS-NS-3004 } \\
\text { ASTM } 5174-91 \\
\text { EPA } 904.0 \\
\text { EPA } 904.0 \\
\text { EPA } 300.0 \\
\text { EPA } 160.1 \\
\text { EPA } 415.1 \\
\text { EPA } 9020 \\
\text { EPA CLP } \\
\text { EPA CLP } \\
\text { EPA CLP } \\
\text { EPA CLP } \\
\text { EPA CLP } \\
\text { EPA CLP } \\
\text { EPA CLP } \\
\text { EPA CLP } \\
\text { EPA CLP } \\
\text { EPA CLP } \\
\text { EPA CLP } \\
\text { EPA CLP } \\
\text { EPA CLP } \\
\text { EPA CLP } \\
\text { EPA CLP } \\
\text { EPA CLP } \\
\text { EPA CLP } \\
\text { EPA CLP } \\
\text { EPA CLP } \\
\text { EPA CLP } \\
\text { EPA CLP } \\
\text { EPA CLP } \\
\text { EPA CLP } \\
\text { EPA CLP } \\
\text { EPA CLP } \\
\text { EPA CLP } \\
\text { EPA CLP } \\
\text { EPA CLP } \\
\text { EPA CLP } \\
\text { EPA CLP } \\
\text { EPA CLP } \\
\text { EPA CLP } \\
\text { EPA CLP } \\
\text { EPA CLP } \\
\text { EPA } 410.4 \\
\text { EPA } 353.1 \\
\end{array}$ \\
\hline
\end{tabular}




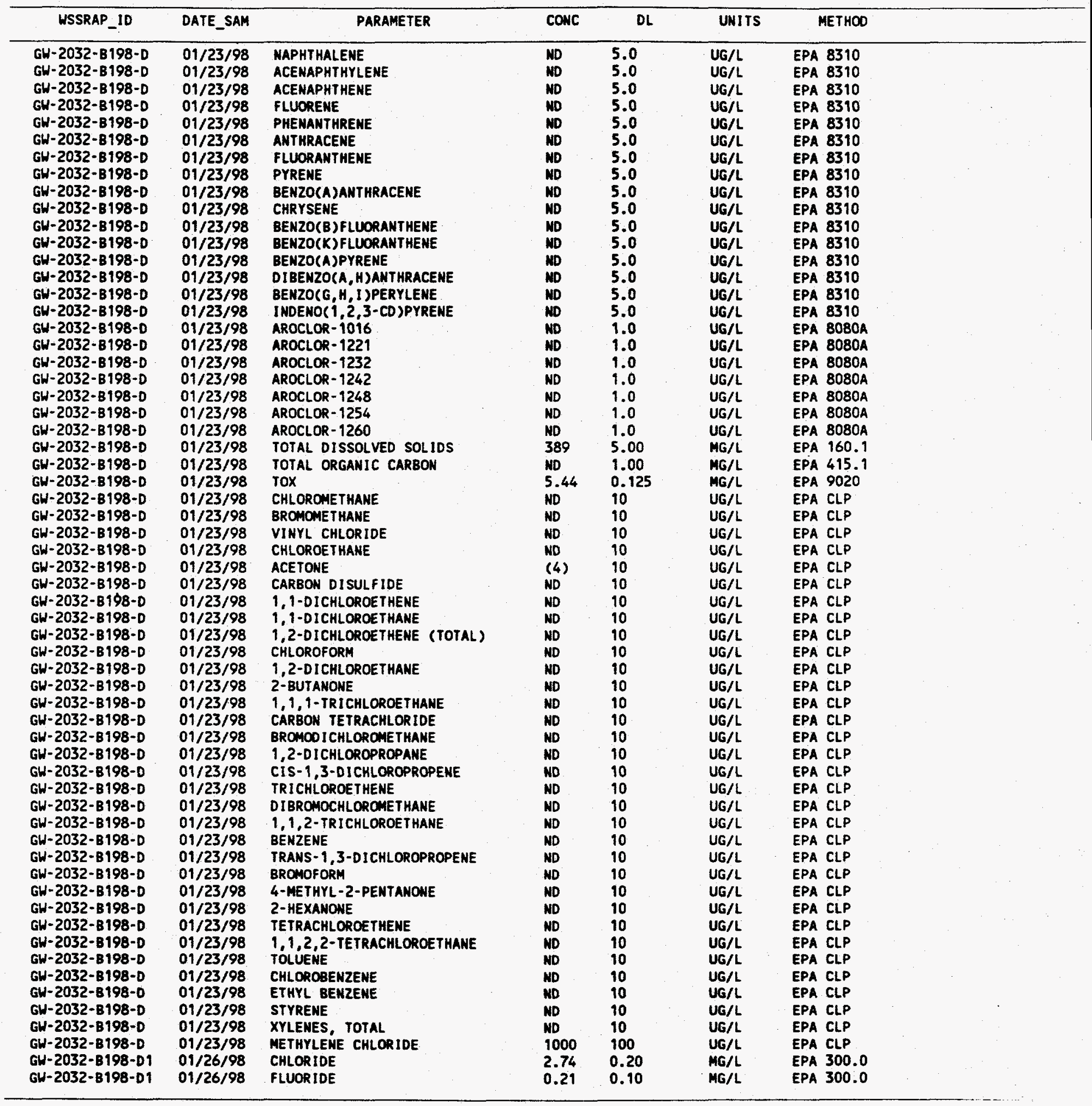




\begin{tabular}{|c|c|c|c|c|c|c|}
\hline WSSRAP_ID & DATE_SAM & PARAMETER & CONC & $\mathrm{DL}$ & UNITS & METHOD \\
\hline 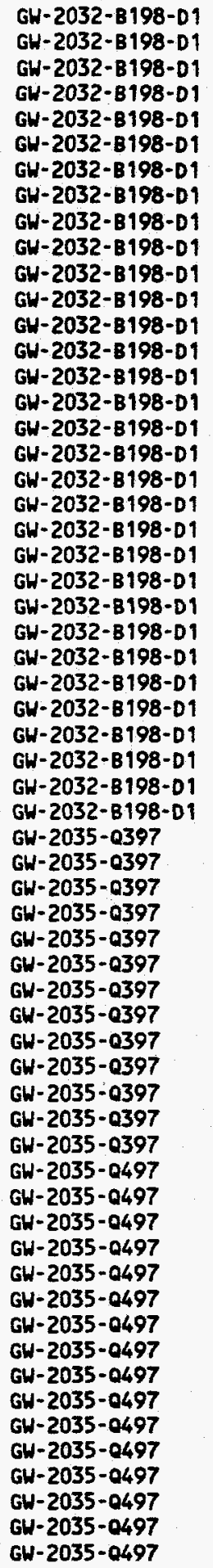 & $\begin{array}{l}01 / 26 / 98 \\
01 / 26 / 98 \\
01 / 26 / 98 \\
01 / 26 / 98 \\
01 / 26 / 98 \\
01 / 26 / 98 \\
01 / 26 / 98 \\
01 / 26 / 98 \\
01 / 26 / 98 \\
01 / 26 / 98 \\
01 / 26 / 98 \\
01 / 26 / 98 \\
01 / 26 / 98 \\
01 / 26 / 98 \\
01 / 26 / 98 \\
01 / 26 / 98 \\
01 / 26 / 98 \\
01 / 26 / 98 \\
01 / 26 / 98 \\
01 / 26 / 98 \\
01 / 26 / 98 \\
01 / 26 / 98 \\
01 / 26 / 98 \\
01 / 26 / 98 \\
01 / 26 / 98 \\
01 / 26 / 98 \\
01 / 26 / 98 \\
01 / 26 / 98 \\
01 / 26 / 98 \\
01 / 26 / 98 \\
01 / 26 / 98 \\
01 / 26 / 98 \\
09 / 17 / 97 \\
09 / 17 / 97 \\
09 / 17 / 97 \\
09 / 17 / 97 \\
09 / 17 / 97 \\
09 / 17 / 97 \\
09 / 17 / 97 \\
09 / 17 / 97 \\
09 / 17 / 97 \\
09 / 17 / 97 \\
09 / 17 / 97 \\
09 / 17 / 97 \\
09 / 17 / 97 \\
11 / 17 / 97 \\
111 / 17 / 97 \\
11 / 17 / 97 \\
11 / 17 / 97 \\
11 / 17 / 97 \\
11117 / 97 \\
11 / 17 / 97 \\
11 / 17 / 97 \\
11117 / 97 \\
11 / 17 / 97 \\
111 / 17 / 97 \\
11 / 17 / 97 \\
11 / 17 / 97 \\
11 / 17 / 97 \\
111 / 17 / 97 \\
111 / 17 / 97\end{array}$ & 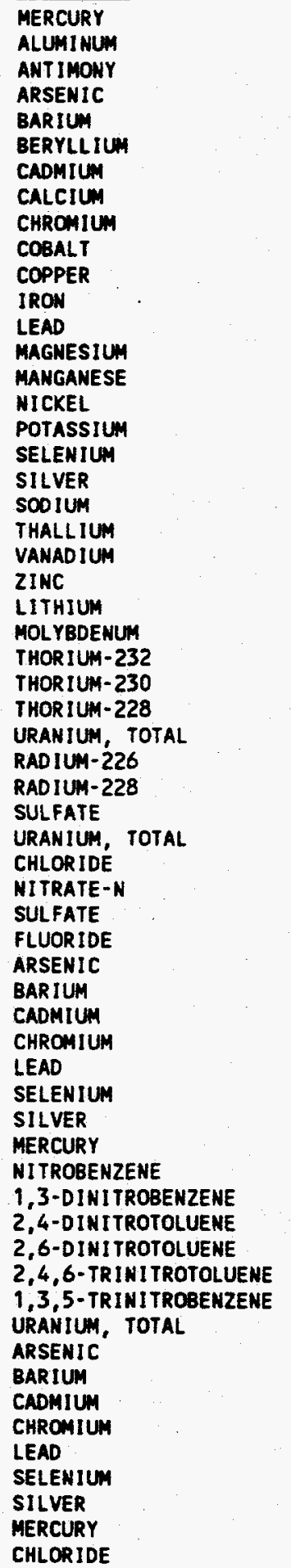 & $\begin{array}{l}\text { ND } \\
169 \\
\text { ND } \\
\text { ND } \\
147 \\
\text { ND } \\
\text { ND } \\
60000 \\
5.5 \\
\text { ND } \\
4.4 \\
311 \\
1.1 \\
30000 \\
20.0 \\
\text { ND } \\
\text { ND } \\
\text { ND } \\
\text { ND } \\
12600 \\
\text { ND } \\
9.7 \\
13.2 \\
12.0 \\
\text { ND } \\
60.04 \\
0.733 \\
60.05 \\
2.43 \\
0.212 \\
60.12 \\
15.9 \\
60.39 \\
0.839 \\
0.263 \\
1.72 \\
1.48 \\
\text { ND } \\
91.7 \\
\text { ND } \\
\text { ND } \\
\text { ND } \\
\text { ND } \\
\text { ND } \\
\text { ND } \\
\text { ND } \\
\text { ND } \\
\text { ND } \\
\text { ND } \\
\text { ND } \\
\text { ND } \\
0.406 \\
\text { ND } \\
88.6 \\
\text { ND } \\
\text { ND } \\
\text { ND } \\
\text { ND } \\
\text { ND } \\
0.812\end{array}$ & $\begin{array}{l}0.10 \\
31.3 \\
42.5 \\
1.3 \\
0.40 \\
0.40 \\
3.3 \\
15.9 \\
2.7 \\
4.3 \\
4.1 \\
5.9 \\
0.90 \\
0\end{array}$ & $\begin{array}{l}\text { UG/L } \\
U G / L \\
U G / L \\
U G / L \\
U G / L \\
U G / L \\
U G / L \\
U G / L \\
U G / L \\
U G / L \\
U G / L \\
U G / L \\
U G / L \\
U G / L \\
U G / L \\
U G / L \\
U G / L \\
U G / L \\
U G / L \\
U G / L \\
U G / L \\
U G / L \\
U G / L \\
U G / L \\
U G / L \\
P C I / L \\
P C I / L \\
P C I / L \\
P C I / L \\
P C I / L \\
P C I / L \\
M G / L \\
P C I / L \\
M G / L \\
M G / L \\
M G / L \\
M G / L \\
U G / L \\
U G / L \\
U G / L \\
U G / L \\
U G / L \\
U G / L \\
U G / L \\
U G / L \\
U G / L \\
U G / L \\
U G / L \\
U G / L \\
U G / L \\
U G / L \\
P C I / L \\
U G / L \\
U G / L \\
U G / L \\
U G / L \\
U G / L \\
U G / L \\
U G / L \\
U G / L \\
U G / L\end{array}$ & $\begin{array}{l}\text { EPA CLP } \\
\text { EPA CLP } \\
\text { EPA CLP } \\
\text { EPA CLP } \\
\text { EPA CLP } \\
\text { EPA CLP } \\
\text { EPA CLP } \\
\text { EPA CLP } \\
\text { EPA CLP } \\
\text { EPA CLP } \\
\text { EPA CLP } \\
\text { EPA CLP } \\
\text { EPA CLP } \\
\text { EPA CLP } \\
\text { EPA CLP } \\
\text { EPA CLP } \\
\text { EPA CLP } \\
\text { EPA CLP } \\
\text { EPA CLP } \\
\text { EPA CLP } \\
\text { EPA CLP } \\
\text { EPA CLP } \\
\text { EPA CLP } \\
\text { EPA CLP } \\
\text { EPA CLP } \\
\text { NAS-NS-3004 } \\
\text { NAS-NS-3004 } \\
\text { NAS-NS-3004 } \\
\text { ASTM } 5174-91 \\
\text { EPA } 904.0 \\
\text { EPA } 904.0 \\
\text { EPA } 300.0 \\
\text { ASTM } 5174-91 \\
\text { EPA } 300.0 \\
\text { EPA } 300.0 \\
\text { EPA } 300.0 \\
\text { EPA } 340.2 \\
\text { EPA CLP } \\
\text { EPA CLP } \\
\text { EPA CLP } \\
\text { EPA CLP } \\
\text { EPA CLP } \\
\text { EPA CLP } \\
\text { EPA CLP } \\
\text { EPA CLP } \\
\text { USATHAMA } \\
\text { USATHAMA } \\
\text { USATHAMA } \\
\text { USATHAMA } \\
\text { USATHAMA } \\
\text { USATHAMA } \\
\text { EPA } 6020 \\
\text { EPA CLP } \\
\text { EPA CLP } \\
\text { EPA CLP } \\
\text { EPA CLP } \\
\text { EPA CLP } \\
\text { EPA CLP } \\
\text { EPA CLP } \\
\text { EPA CLP } \\
\text { EPA } 300.0 \\
\end{array}$ \\
\hline
\end{tabular}




\begin{tabular}{|c|c|c|c|c|c|c|}
\hline WSSRAP_ID & DATE_SAM & PARAMETER & CONC & DL & UNITS & METHOO \\
\hline $\begin{array}{l}\text { GW-2035-0497 } \\
G W-2035-0497 \\
G W-2035-0497 \\
G W-2036-0397 \\
G W-2036-0397 \\
G W-2036-0397 \\
G W-2036-0397 \\
G W-2036-0397 \\
G W-2036-0397 \\
G W-2036-0397 \\
G W-2036-0397 \\
G W-2036-0397 \\
G W-2036-0397 \\
G W-2036-0397 \\
G W-2036-0397 \\
G W-2036-0397 \\
G W-2036-0497 \\
G W-2036-0497 \\
G W-2036-0497 \\
G W-2036-0497 \\
G W-2036-0497 \\
G W-2036-0497 \\
G W-2036-0497 \\
G W-2036-0497 \\
G W-2036-0497 \\
G W-2036-0497 \\
G W-2036-0497 \\
G W-2036-0497 \\
G W-2036-0497 \\
G W-2036-0497 \\
G W-2036-0497 \\
G W-2036-0497 \\
G W-2036-0497 \\
G W-2036-0497 \\
G W-2036-0497 \\
G W-2036-021898 \\
G W-2036-021898 \\
G W-2037-1097 \\
G W-2037-1097 \\
G W-2037-1097 \\
G W-2037-1097 \\
G W-2037-1097 \\
G W-2037-1097 \\
G W-2037-1097 \\
G W-2037-1097 \\
G W-2037-1097 \\
G W-2037-1097 \\
G W-2037-1097 \\
G W-2037-1097 \\
G W-2037-1097 \\
G W-2037-1097 \\
G W-2037-1097 \\
G W-2037-1097 \\
G W-2037-1097 \\
G W-2037-1097 \\
G W-2037-1097 \\
G W-2037-1197 \\
G W-2037-1197 \\
G W-2037-1197 \\
G W-2037-1197 \\
G W-2037-11997\end{array}$ & $\begin{array}{l}11 / 17 / 97 \\
11 / 17 / 97 \\
11 / 17 / 97 \\
09 / 17 / 97 \\
09 / 17 / 97 \\
09 / 17 / 97 \\
09 / 17 / 97 \\
09 / 17 / 97 \\
09 / 17 / 97 \\
09 / 17 / 97 \\
09 / 17 / 97 \\
09 / 17 / 97 \\
09 / 17 / 97 \\
09 / 17 / 97 \\
09 / 17 / 97 \\
09 / 17 / 97 \\
11 / 17 / 97 \\
11 / 17 / 97 \\
11 / 17 / 97 \\
11 / 17 / 97 \\
11 / 17 / 97 \\
11 / 17 / 97 \\
11 / 17 / 97 \\
11 / 17 / 97 \\
11 / 17 / 97 \\
11 / 17 / 97 \\
11 / 17 / 97 \\
11 / 17 / 97 \\
11 / 17 / 97 \\
11 / 17 / 97 \\
11 / 17 / 97 \\
11 / 17 / 97 \\
11 / 17 / 97 \\
11 / 17 / 97 \\
11 / 17 / 97 \\
02 / 18 / 98 \\
02 / 18 / 98 \\
10 / 23 / 97 \\
10 / 23 / 97 \\
10 / 23 / 97 \\
10 / 23 / 97 \\
10 / 23 / 97 \\
10 / 23 / 97 \\
10 / 23 / 97 \\
10 / 23 / 97 \\
10 / 23 / 97 \\
10 / 23 / 97 \\
10 / 23 / 97 \\
10 / 23 / 97 \\
10 / 23 / 97 \\
10 / 23 / 97 \\
10 / 23 / 97 \\
10 / 23 / 97 \\
10 / 23 / 97 \\
10 / 23 / 97 \\
10 / 23 / 97 \\
11 / 17 / 97 \\
111117 / 97 \\
111 / 17 / 97 \\
11 / 17 / 97 \\
11 / 17 / 97\end{array}$ & 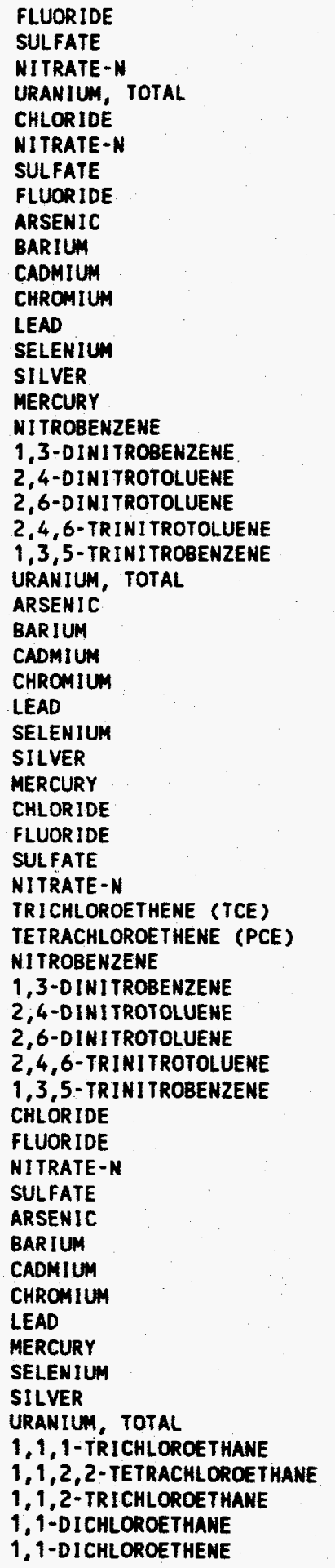 & 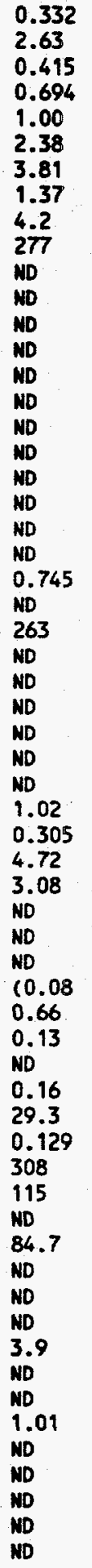 & $\begin{array}{l}0.200 \\
1.00 \\
0.0100 \\
0.42 \\
0.020 \\
0 \\
0.020 \\
0.10 \\
0\end{array}$ & 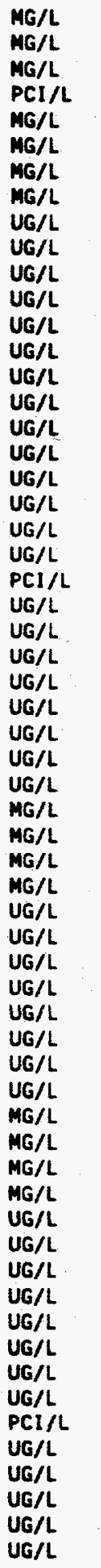 & $\begin{array}{l}\text { EPA } 300.0 \\
\text { EPA } 300.0 \\
\text { EPA } 353.2 \\
\text { ASTM } 5174-91 \\
\text { EPA } 300.0 \\
\text { EPA } 300.0 \\
\text { EPA } 300.0 \\
\text { EPA } 340.2 \\
\text { EPA CLP } \\
\text { EPA CLP } \\
\text { EPA CLP } \\
\text { EPA CLP } \\
\text { EPA CLP } \\
\text { EPA CLP } \\
\text { EPA CLP } \\
\text { EPA CLP } \\
\text { USATHAMA } \\
\text { USATHAMA } \\
\text { USATHAMA } \\
\text { USATHAMA } \\
\text { USATHAMA } \\
\text { USATHAMA } \\
\text { EPA } 6020 \\
\text { EPA CLP } \\
\text { EPA CLP } \\
\text { EPA CLP } \\
\text { EPA CLP } \\
\text { EPA CLP } \\
\text { EPA CLP } \\
\text { EPA CLP } \\
\text { EPA CLP } \\
\text { EPA } 300.0 \\
\text { EPA } 300.0 \\
\text { EPA } 300.0 \\
\text { EPA } 353.2 \\
\text { EPA } 8260 \\
\text { EPA } 8260 \\
\text { USATHAMA } \\
\text { USATHAMA } \\
\text { USATHAMA } \\
\text { USATHAMA } \\
\text { USATHAMA } \\
\text { USATHAMA } \\
\text { EPA } 300.0 \\
\text { EPA } 340.2 \\
\text { EPA } 353.2 \\
\text { EPA } 300.0 \\
\text { EPA CLP } \\
\text { EPA CLP } \\
\text { EPA CLP } \\
\text { EPA CLP } \\
\text { EPA CLP } \\
\text { EPA CLP } \\
\text { EPA CLP } \\
\text { EPA CLP } \\
\text { ASTM } 5174-91 \\
\text { EPA CLP } \\
\text { EPA CLPP } \\
\text { EPA CLP } \\
\text { EPA CLP } \\
\text { EPA CLP } \\
\end{array}$ \\
\hline
\end{tabular}




\begin{tabular}{|c|c|c|c|c|c|c|}
\hline WSSF & DATE_SAM & PARAMETER & CONC & $D L$ & UNITS & METHOD \\
\hline $\begin{array}{l}\text { GW-2037-1197 } \\
\text { GW-2037-1197 } \\
G W-2037-1197 \\
G W-2037-1197 \\
G W-2037-1197 \\
G W-2037-1197 \\
G W-2037-1197 \\
G W-2037-1197 \\
G W-2037-1197 \\
G W-2037-1197 \\
G W-2037-1197 \\
G W-2037-1197 \\
G W-2037-1197 \\
G W-2037-1197 \\
G W-2037-1197 \\
G W-2037-1197 \\
G W-2037-1197 \\
G W-2037-1197 \\
G W-2037-1197 \\
G W-2037-1197 \\
G W-2037-1197 \\
G W-2037-1197 \\
G W-2037-1197 \\
G W-2037-1197 \\
G W-2037-1197 \\
G W-2037-1197 \\
G W-2037-1197 \\
G W-2037-1197 \\
G W-2037-1197 \\
G W-2037-1297 \\
G W-2037-1297 \\
G W-2037-1297 \\
G W-2037-1297 \\
G W-2037-1297 \\
G W-2037-1297 \\
G W-2037-1297 \\
G W-2037-1297 \\
G W-2037-1297 \\
G W-2037-1297 \\
G W-2037-1297 \\
G W-2037-1297 \\
G W-2037-1297 \\
G W-2037-11297 \\
G W-2037-1297 \\
G W-2037-1297 \\
G W-2037-1297 \\
G W-2037-1297 \\
G W-2037-1297 \\
G W-2037-1297 \\
G W-2037-12037-1297 \\
G W-2037-1297 \\
G W-2037-1297 \\
G W-2037-1297 \\
G W-2037-1297 \\
G-2037-1297 \\
G-2037-1297 \\
\text { GW }\end{array}$ & $\begin{array}{l}11 / 17 / 97 \\
11 / 17 / 97 \\
11 / 17 / 97 \\
11 / 17 / 97 \\
11 / 17 / 97 \\
11 / 17 / 97 \\
11 / 17 / 97 \\
11 / 17 / 97 \\
11 / 17 / 97 \\
11 / 17 / 97 \\
11 / 17 / 97 \\
11 / 17 / 97 \\
11 / 17 / 97 \\
11 / 17 / 97 \\
11 / 17 / 97 \\
11 / 17 / 97 \\
11 / 17 / 97 \\
11 / 17 / 97 \\
11 / 17 / 97 \\
11 / 17 / 97 \\
11 / 17 / 97 \\
11 / 17 / 97 \\
11 / 17 / 97 \\
11 / 17 / 97 \\
11 / 17 / 97 \\
11 / 17 / 97 \\
11 / 17 / 97 \\
11 / 17 / 97 \\
11 / 17 / 97 \\
12 / 02 / 97 \\
12 / 02 / 97 \\
12 / 02 / 97 \\
12 / 02 / 97 \\
12 / 02 / 97 \\
12 / 02 / 97 \\
12 / 02 / 97 \\
12 / 02 / 97 \\
12 / 02 / 97 \\
12 / 02 / 97 \\
12 / 02 / 97 \\
12 / 02 / 97 \\
12 / 02 / 97 \\
12 / 02 / 97 \\
12 / 02 / 97 \\
12 / 02 / 97 \\
12 / 02 / 97 \\
12 / 02 / 97 \\
12 / 02 / 97 \\
12102 / 97 \\
12 / 02 / 97 \\
12 / 02 / 97 \\
12 / 02 / 97 \\
12 / 02 / 97 \\
12 / 02 / 97 \\
12 / 02 / 97 \\
12 / 02 / 97 \\
12 / 02 / 97 \\
12 / 02 / 97 \\
12 / 02 / 97 \\
12 / 02 / 97 \\
12 / 02 / 97\end{array}$ & 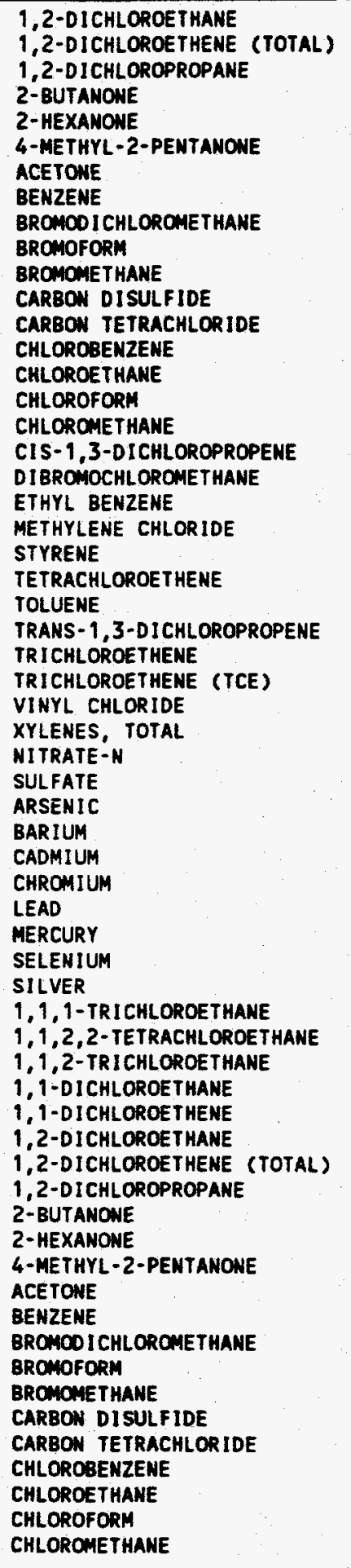 & 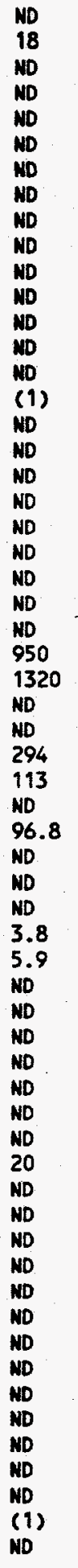 & $\begin{array}{l}10 \\
10 \\
10 \\
10 \\
10 \\
10 \\
10 \\
10 \\
10 \\
10 \\
10 \\
10 \\
10 \\
10 \\
10 \\
10 \\
10 \\
10 \\
10 \\
10 \\
10 \\
10 \\
10 \\
10 \\
10 \\
10 \\
10 \\
10 \\
10 \\
10\end{array}$ & $\begin{array}{l}U G / L \\
U G / L \\
U G / L \\
U G / L \\
U G / L \\
U G / L \\
U G / L \\
U G / L \\
U G / L \\
U G / L \\
U G / L \\
U G / L \\
U G / L \\
U G / L \\
U G / L \\
U G / L \\
U G / L \\
U G / L \\
U G / L \\
U G / L \\
U G / L \\
U G / L \\
U G / L \\
U G / L \\
U G / L \\
U G / L \\
U G / L \\
U G / L \\
U G / L \\
M G / L \\
U G / L \\
U G / L \\
U G / L \\
U G / L \\
U G / L \\
U G / L \\
U G / L \\
U G / L \\
U G / L \\
U G / L \\
U G / L \\
U G / L \\
U G / L \\
U G / L \\
U G / L \\
U G / L \\
U G / L \\
U G / L \\
U G / L \\
U G / L \\
U G / L \\
U G / L \\
U G / L \\
U G / L \\
U G / L \\
U G / L \\
U G / L \\
U G \\
U S\end{array}$ & $\begin{array}{l}\text { EPA CLP } \\
\text { EPA CLP } \\
\text { EPA CLP } \\
\text { EPA CLP } \\
\text { EPA CLP } \\
\text { EPA CLP } \\
\text { EPA CLP } \\
\text { EPA CLP } \\
\text { EPA CLP } \\
\text { EPA CLP } \\
\text { EPA CLP } \\
\text { EPA CLP } \\
\text { EPA CLP } \\
\text { EPA CLP } \\
\text { EPA CLP } \\
\text { EPA CLP } \\
\text { EPA CLP } \\
\text { EPA CLP } \\
\text { EPA CLP } \\
\text { EPA CLP } \\
\text { EPA CLP } \\
\text { EPA CLP } \\
\text { EPA CLP } \\
\text { EPA CLP } \\
\text { EPA CLP } \\
\text { EPA CLP } \\
\text { EPA } 601 \\
\text { EPA CLP } \\
\text { EPA CLP } \\
\text { EPA } 353.1 \\
\text { EPA } 300.0 \\
\text { EPA CLP } \\
\text { EPA CLP } \\
\text { EPA CLP } \\
\text { EPA CLP } \\
\text { EPA CLP } \\
\text { EPA CLP } \\
\text { EPA CLP } \\
\text { EPA CLP } \\
\text { EPA CLP } \\
\text { EPA CLP } \\
\text { EPA CLP } \\
\text { EPA CLP } \\
\text { EPA CLP } \\
\text { EPA CLP } \\
\text { EPA CLP } \\
\text { EPA CLP } \\
\text { EPA CLP } \\
\text { EPA CLP } \\
\text { EPA CLP } \\
\text { EPA CLP } \\
\text { EPA CLP } \\
\text { EPA CLP } \\
\text { EPA CLP } \\
\text { EPA CLP } \\
\text { EPA CLP } \\
\text { EPA CLP } \\
\text { EPA CLP } ~ C L P ~ \\
\text { EPA CLP } \\
\text { CLA }\end{array}$ \\
\hline
\end{tabular}




\begin{tabular}{|c|c|c|c|c|c|c|}
\hline USSRA & DATE_SAM & PARAMETER & CONC & DL & UNITS & METHOO \\
\hline $\begin{array}{l}\text { GW-2037-1297 } \\
\text { GW-2037-1297 } \\
\text { GW-2037-1297 } \\
\text { GW-2037-1297 } \\
G W-2037-1297 \\
G W-2037-1297 \\
G W-2037-1297 \\
G W-2037-1297 \\
G W-2037-1297 \\
G W-2037-1297 \\
G W-2037-1297 \\
G W-2037-1297 \\
G W-2037-021898 \\
G W-2037-021898 \\
G W-2038-1097 \\
G W-2038-1097 \\
G W-2038-1097 \\
G W-2038-1097 \\
G W-2038-1097 \\
G W-2038-1097 \\
G W-2038-1097 \\
G W-2038-1097 \\
G W-2038-1097 \\
G W-2038-1097 \\
G W-2038-1097 \\
G W-2038-1097 \\
G W-2038-1097 \\
G W-2038-1097 \\
G W-2038-1097 \\
G W-2038-1097 \\
G W-2038-1097 \\
G W-2038-1097 \\
G W-2038-1097 \\
G W-2038-1197 \\
G W-2038-1197 \\
G W-2038-1197 \\
G W-2038-1197 \\
G W-2038-1197 \\
G W-2038-1197 \\
G W-2038-1197 \\
G W-2038-1197 \\
G W-2038-1197 \\
G W-2038-1197 \\
G W-2038-1197 \\
G W-2038-1197 \\
G W-2038-1197 \\
G W-2038-1197 \\
G W-2038-1197 \\
G W-2038-1197 \\
G W-2038-1197 \\
G W-2038-1197 \\
G W-2038-1197 \\
G W-2038-1197 \\
G W-2038-1197 \\
G W-2038-1197 \\
G W-2038-1197 \\
G W-2038-1197 \\
G W-2038-1197 \\
G W-2038-1197 \\
G W-2038-1197 \\
G W-2038-1197\end{array}$ & $\begin{array}{l}12 / 02 / 97 \\
12 / 02 / 97 \\
12 / 02 / 97 \\
12 / 02 / 97 \\
12 / 02 / 97 \\
12 / 02 / 97 \\
12 / 02 / 97 \\
12 / 02 / 97 \\
12 / 02 / 97 \\
12 / 02 / 97 \\
12 / 02 / 97 \\
12 / 02 / 97 \\
02 / 18 / 98 \\
02 / 18 / 98 \\
10 / 23 / 97 \\
10 / 23 / 97 \\
10 / 23 / 97 \\
10 / 23 / 97 \\
10 / 23 / 97 \\
10 / 23 / 97 \\
10 / 23 / 97 \\
10 / 23 / 97 \\
10 / 23 / 97 \\
10 / 23 / 97 \\
10 / 23 / 97 \\
10 / 23 / 97 \\
10 / 23 / 97 \\
10 / 23 / 97 \\
10 / 23 / 97 \\
10 / 23 / 97 \\
10 / 23 / 97 \\
10 / 23 / 97 \\
10 / 23 / 97 \\
11 / 18 / 97 \\
11 / 18 / 97 \\
11 / 18 / 97 \\
11 / 18 / 97 \\
11 / 18 / 97 \\
11 / 18 / 97 \\
11 / 18 / 97 \\
11 / 18 / 97 \\
11 / 18 / 97 \\
11 / 18 / 97 \\
11 / 18 / 97 \\
11 / 18 / 97 \\
11 / 18 / 97 \\
11 / 18 / 97 \\
111 / 18 / 97 \\
11 / 18 / 97 \\
11 / 18 / 97 \\
11 / 18 / 97 \\
11 / 18 / 97 \\
111 / 18 / 97 \\
11 / 18 / 97 \\
11 / 18 / 97 \\
11 / 18 / 97 \\
11 / 18 / 97 \\
11 / 18 / 97 \\
11 / 18 / 97 \\
11 / 18 / 97 \\
11 / 18 / 97\end{array}$ & 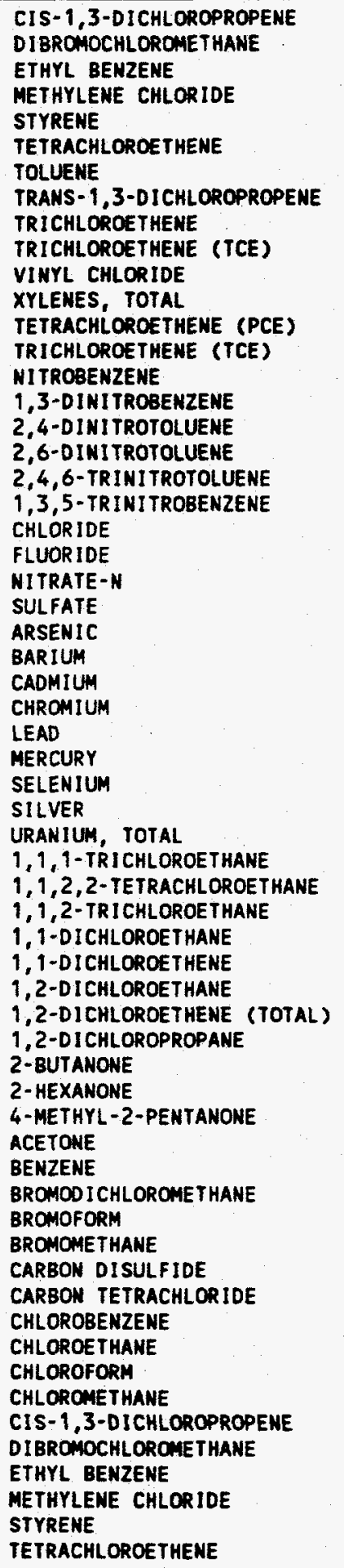 & $\begin{array}{l}\text { ND } \\
\text { ND } \\
\text { ND } \\
\text { ND } \\
\text { ND } \\
\text { ND } \\
\text { ND } \\
\text { ND } \\
1200 \\
1290 \\
\text { ND } \\
\text { ND } \\
1 \\
1200 \\
\text { ND } \\
\text { CO.06 } \\
1.2 \\
\text { O.24 } \\
\text { ND } \\
\text { O.14 } \\
21 \\
0.092 \\
827 \\
72.5 \\
\text { ND } \\
228 \\
\text { ND } \\
\text { ND } \\
\text { ND } \\
\text { O.92 } \\
11.1 \\
\text { ND } \\
1.55 \\
\text { ND } \\
\text { ND } \\
\text { ND } \\
\text { ND } \\
\text { ND } \\
\text { ND } \\
12 \\
\text { ND } \\
\text { ND } \\
\text { ND } \\
\text { ND } \\
\text { HD } \\
\text { ND } \\
\text { ND } \\
\text { ND } \\
\text { ND } \\
\text { ND } \\
\text { ND } \\
\text { ND } \\
\text { ND } \\
\text { ND } \\
\text { ND } \\
\text { ND } \\
\text { ND } \\
\text { ND } \\
\text { ND } \\
\text { ND } \\
\text { ND }\end{array}$ & $\begin{array}{l}10 \\
10 \\
10 \\
10 \\
10 \\
10 \\
10 \\
10 \\
500 \\
100 \\
10 \\
10 \\
1 \\
50 \\
0.060 \\
0.090 \\
0.030 \\
0.010 \\
0.030 \\
0.030 \\
0.010 \\
0.030 \\
2.5 \\
0.030 \\
3.0 \\
1.0 \\
1.0 \\
1.0 \\
2.0 \\
0.20 \\
4.0 \\
1.0 \\
0.42 \\
10 \\
10 \\
10 \\
10 \\
10 \\
10 \\
10 \\
10 \\
10 \\
10 \\
10 \\
10 \\
10 \\
10 \\
10 \\
10 \\
10 \\
10 \\
10 \\
10 \\
10 \\
10 \\
10 \\
10 \\
10 \\
10 \\
10 \\
10 \\
10 \\
10 \\
10 \\
10 \\
10 \\
10\end{array}$ & 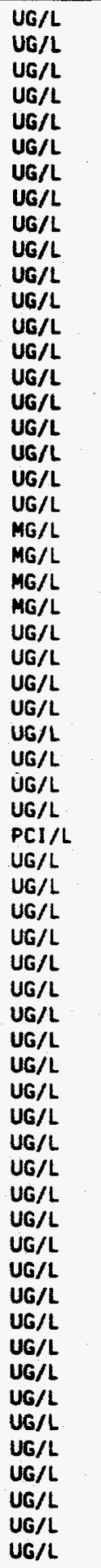 & $\begin{array}{l}\text { EPA CLP } \\
\text { EPA CLP } \\
\text { EPA CLP } \\
\text { EPA CLP } \\
\text { EPA CLP } \\
\text { EPA CLP } \\
\text { EPA CLP } \\
\text { EPA CLP } \\
\text { EPA CLP } \\
\text { EPA } 601 \\
\text { EPA CLP } \\
\text { EPA CLP } \\
\text { EPA } 8260 \\
\text { EPA } 8260 \\
\text { USATHAMA } \\
\text { USATHAMA } \\
\text { USATHAMA } \\
\text { USATHAMA } \\
\text { USATHAMA } \\
\text { USATHAMA } \\
\text { EPA } 300.0 \\
\text { EPA } 340.2 \\
\text { EPA } 353.2 \\
\text { EPA } 300.0 \\
\text { EPA CLP } \\
\text { EPA CLP } \\
\text { EPA CLP } \\
\text { EPA CLP } \\
\text { EPA CLP } \\
\text { EPA CLP } \\
\text { EPA CLP } \\
\text { EPA CLP } \\
\text { ASTM } 5174-91 \\
\text { EPA CLP } \\
\text { EPA CLP } \\
\text { EPA CLP } \\
\text { EPA CLP } \\
\text { EPA CLP } \\
\text { EPA CLP } \\
\text { EPA CLP } \\
\text { EPA CLP } \\
\text { EPA CLP } \\
\text { EPA CLP } \\
\text { EPA CLP } \\
\text { EPA CLP } \\
\text { EPA CLP } \\
\text { EPA CLP } \\
\text { EPA CLP } \\
\text { EPA CLP } \\
\text { EPA CLP } \\
\text { EPA CLP } \\
\text { EPA CLP } \\
\text { EPA CLP } \\
\text { EPA CLP } \\
\text { EPA CLP } \\
\text { EPA CLP } \\
\text { EPA CLP } \\
\text { EPA CLP } \\
\text { EPA CLP } \\
\text { EPA CLP } \\
\text { EPA CLP } \\
\end{array}$ \\
\hline
\end{tabular}




\begin{tabular}{|c|c|c|c|c|c|c|}
\hline WSSRAP_ID & DATE_SAM & PARAMETER & Conc & $\mathrm{DL}$ & UNITS & METHOD \\
\hline 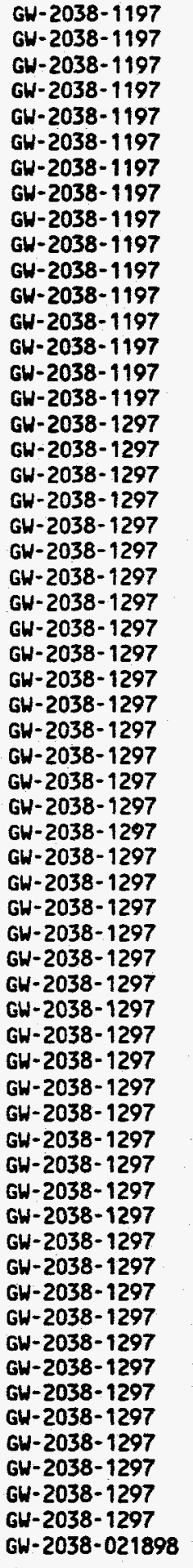 & $\begin{array}{l}11 / 18 / 97 \\
11 / 18 / 97 \\
11 / 18 / 97 \\
11 / 18 / 97 \\
11 / 18 / 97 \\
11 / 18 / 97 \\
11 / 18 / 97 \\
11 / 18 / 97 \\
11 / 18 / 97 \\
11 / 18 / 97 \\
11 / 18 / 97 \\
11 / 18 / 97 \\
11 / 18 / 97 \\
11 / 18 / 97 \\
11 / 18 / 97 \\
11 / 18 / 97 \\
12 / 02 / 97 \\
12 / 02 / 97 \\
12 / 02 / 97 \\
12 / 02 / 97 \\
12 / 02 / 97 \\
12 / 02 / 97 \\
12 / 02 / 97 \\
12 / 02 / 97 \\
12 / 02 / 97 \\
12 / 02 / 97 \\
12 / 02 / 97 \\
12 / 02 / 97 \\
12 / 02 / 97 \\
12 / 02 / 97 \\
12 / 02 / 97 \\
12 / 02 / 97 \\
12 / 02 / 97 \\
12 / 02 / 97 \\
12 / 02 / 97 \\
12 / 02 / 97 \\
12 / 02 / 97 \\
12 / 02 / 97 \\
12 / 02 / 97 \\
12 / 02 / 97 \\
12 / 02 / 97 \\
12 / 02 / 97 \\
12 / 02 / 97 \\
12 / 02 / 97 \\
12 / 02 / 97 \\
12 / 02 / 97 \\
12 / 02 / 97 \\
12 / 02 / 97 \\
12 / 02 / 97 \\
12 / 02 / 97 \\
12 / 02 / 97 \\
12 / 02 / 97 \\
12 / 02 / 97 \\
12 / 02 / 97 \\
12 / 02 / 97 \\
12 / 02 / 97 \\
12 / 02 / 97 \\
12 / 02 / 97 \\
12 / 02 / 97 \\
12 / 02 / 97 \\
02 / 18 / 98\end{array}$ & 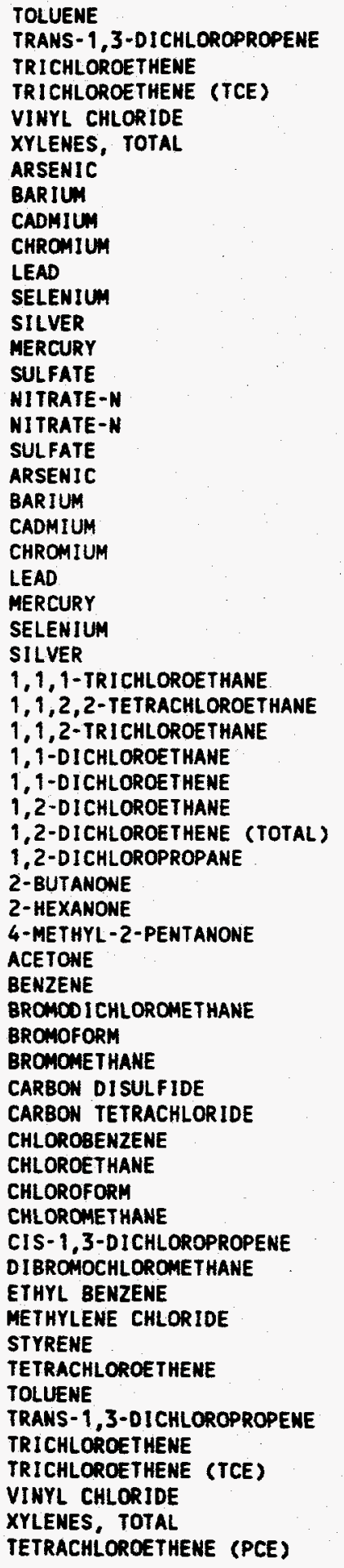 & 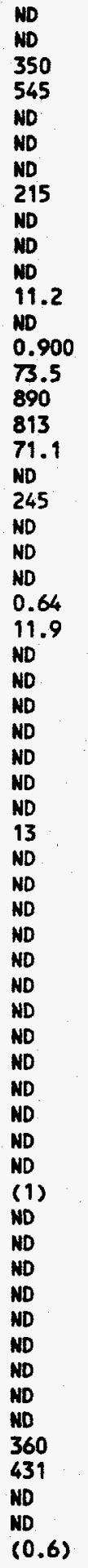 & 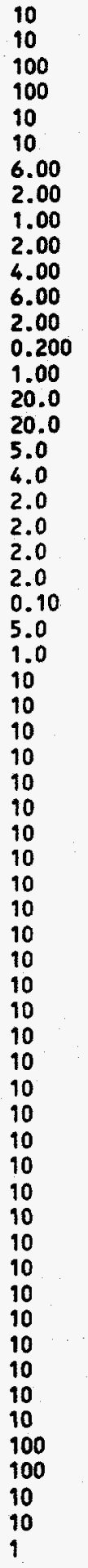 & $\begin{array}{l}U G / L \\
U G / L \\
U G / L \\
U G / L \\
U G / L \\
U G / L \\
U G / L \\
U G / L \\
U G / L \\
U G / L \\
U G / L \\
U G / L \\
U G / L \\
U G / L \\
U G / L \\
\text { UG/L } \\
U G / L \\
\text { UG/L } \\
U G / L \\
U G / L \\
U G / L \\
U G / L \\
U G / L \\
U G / L \\
U G / L \\
U G / L \\
U G / L \\
U G / L \\
U G / L \\
U G / L \\
U G / L \\
U G / L \\
U G / L \\
U G / L \\
U G / L \\
U G / L \\
U G / L \\
U G / L \\
U G / L \\
U G / L \\
U G / L \\
U G / L \\
U G / L \\
U G / L \\
U G / L \\
U G / L \\
U G / L \\
U G / L \\
U G / L \\
U G / L \\
U G / L \\
U G / L \\
U G / L \\
U G / L \\
U G / L \\
U G / L \\
U G / L \\
U G / L \\
U G / L \\
U G / L \\
U G / L \\
\text { US } \\
\text { U. }\end{array}$ & $\begin{array}{l}\text { EPA CLP } \\
\text { EPA CLP } \\
\text { EPA CLP } \\
\text { EPA } 601 \\
\text { EPA CLP } \\
\text { EPA CLP } \\
\text { EPA CLP } \\
\text { EPA CLP } \\
\text { EPA CLP } \\
\text { EPA CLP } \\
\text { EPA CLP } \\
\text { EPA CLP } \\
\text { EPA CLP } \\
\text { EPA CLP } \\
\text { EPA } 300.0 \\
\text { EPA } 353.2 \\
\text { EPA } 353.1 \\
\text { EPA } 300.0 \\
\text { EPA CLP } \\
\text { EPA CLP } \\
\text { EPA CLP } \\
\text { EPA CLP } \\
\text { EPA CLP } \\
\text { EPA CLP } \\
\text { EPA CLP } \\
\text { EPA CLP } \\
\text { EPA CLP } \\
\text { EPA CLP } \\
\text { EPA CLP } \\
\text { EPA CLP } \\
\text { EPA CLP } \\
\text { EPA CLP } \\
\text { EPA CLP } \\
\text { EPA CLP } \\
\text { EPA CLP } \\
\text { EPA CLP } \\
\text { EPA CLP } \\
\text { EPA CLP } \\
\text { EPA CLP } \\
\text { EPA CLP } \\
\text { EPA CLP } \\
\text { EPA CLP } \\
\text { EPA CLP } \\
\text { EPA CLP } \\
\text { EPA CLP } \\
\text { EPA CLP } \\
\text { EPA CLP } \\
\text { EPA CLP } \\
\text { EPA CLP } \\
\text { EPA CLP } \\
\text { EPA CLP } \\
\text { EPA CLP } \\
\text { EPA CLP } \\
\text { EPA CLP } \\
\text { EPA CLP } \\
\text { EPA CLP } \\
\text { EPA CLP } \\
\text { EPA } 601 \\
\text { EPA CLP } \\
\text { EPA CLP } \\
\text { EPA } 8260 \\
\end{array}$ \\
\hline
\end{tabular}




\begin{tabular}{|c|c|c|c|c|c|c|}
\hline WSSRAP_ID & DATE_SAM & PARAMETER & CONC & DL & UNITS & METHOD \\
\hline $\begin{array}{l}G W-2038-021898 \\
G H-2039-1097 \\
G W-2039-1097 \\
G W-2039-1097 \\
G W-2039-1097 \\
G W-2039-1097 \\
G W-2039-1097 \\
G W-2039-1097 \\
G W-2039-1097 \\
G W-2039-1097 \\
G W-2039-1097 \\
G W-2039-1297 \\
G W-2039-1297 \\
G W-2039-1297 \\
G W-2039-1297 \\
G W-2039-1297 \\
G W-2039-1297 \\
G W-2039-1297 \\
G W-2039-1297 \\
G W-2039-1297 \\
G W-2039-1297 \\
G W-2039-1297 \\
G W-2039-1297 \\
G W-2039-1297 \\
G W-2039-120897 \\
G W-2039-120897 \\
G W-2039-120897 \\
G W-2039-120897 \\
G W-2039-120897 \\
G W-2039-120897 \\
G W-2040-0397 \\
G W-2040-0397 \\
G W-2040-0397 \\
G W-2040-0397 \\
G W-2040-0397 \\
G W-2040-0397 \\
G W-2040-0397 \\
G W-2040-0397 \\
G W-2040-0397 \\
G W-2040-0397 \\
G W-2040-0397 \\
G W-2040-0397 \\
G W-2040-00397 \\
G W-2040-0497 \\
G W-2040-0497 \\
G W-2040-0497 \\
G W-2040-0497 \\
G W-2040-0497 \\
G W-2040-0497 \\
G W-2040-0497 \\
G W-2040-0497 \\
G W-2040-0497 \\
G W-2040-0497 \\
G W-2040-0497 \\
G W-2040-0497 \\
G W-2040-0497 \\
G W-2040-0497 \\
G W-2040-0497 \\
G W-2040-0497 \\
G W-2040-0497 \\
G W-2040-0497\end{array}$ & $\begin{array}{l}02 / 18 / 98 \\
10 / 23 / 97 \\
10 / 23 / 97 \\
10 / 23 / 97 \\
10 / 23 / 97 \\
10 / 23 / 97 \\
10 / 23 / 97 \\
10 / 23 / 97 \\
10 / 23 / 97 \\
10 / 23 / 97 \\
10 / 23 / 97 \\
12 / 04 / 97 \\
12 / 04 / 97 \\
12 / 04 / 97 \\
12 / 04 / 97 \\
12 / 04 / 97 \\
12 / 04 / 97 \\
12 / 04 / 97 \\
12 / 04 / 97 \\
12 / 04 / 97 \\
12 / 04 / 97 \\
12 / 04 / 97 \\
12 / 04 / 97 \\
12 / 04 / 97 \\
12 / 08 / 97 \\
12 / 08 / 97 \\
12 / 08 / 97 \\
12 / 08 / 97 \\
12 / 08 / 97 \\
12 / 08 / 97 \\
09 / 17 / 97 \\
09 / 17 / 97 \\
09 / 17 / 97 \\
09 / 17 / 97 \\
09 / 17 / 97 \\
09 / 17 / 97 \\
09 / 17 / 97 \\
09 / 17 / 97 \\
09 / 17 / 97 \\
09 / 17 / 97 \\
09 / 17 / 97 \\
09 / 17 / 97 \\
09 / 17 / 97 \\
11 / 18 / 97 \\
11 / 18 / 97 \\
11 / 18 / 97 \\
11 / 18 / 97 \\
11 / 18 / 97 \\
11 / 18 / 97 \\
11 / 18 / 97 \\
11 / 18 / 97 \\
11 / 18 / 97 \\
11 / 18 / 97 \\
11 / 18 / 97 \\
11 / 18 / 97 \\
11 / 18 / 97 \\
11 / 18 / 97 \\
11 / 18 / 97 \\
11 / 18 / 97 \\
11 / 18 / 97 \\
11 / 18 / 97\end{array}$ & 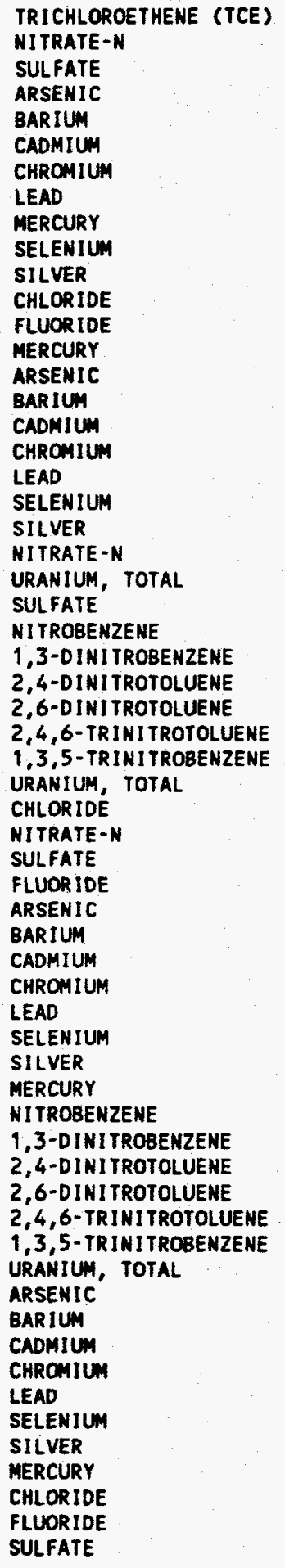 & $\begin{array}{l}390 \\
67.3 \\
28.4 \\
\text { ND } \\
346 \\
\text { ND } \\
3.7 \\
\text { ND } \\
\text { ND } \\
8.2 \\
\text { ND } \\
47.0 \\
0.37 \\
\text { ND } \\
\text { ND } \\
349 \\
\text { ND } \\
3.5 \\
\text { ND } \\
6.2 \\
\text { ND } \\
62.8 \\
2.71 \\
28.6 \\
\text { ND } \\
\text { ND } \\
\text { ND } \\
\text { ND } \\
\text { ND } \\
\text { ND } \\
2.25 \\
2.97 \\
157 \\
11.1 \\
1.15 \\
4.5 \\
638 \\
\text { ND } \\
12.2 \\
2.5 \\
\text { ND } \\
\text { ND } \\
\text { ND } \\
\text { ND } \\
\text { ND } \\
60.01 \\
\text { ND } \\
\text { ND } \\
\text { ND } \\
2.37 \\
\text { ND } \\
630 \\
\text { ND } \\
12.5 \\
2.60 \\
5.80 \\
\text { ND } \\
\text { ND } \\
3.21 \\
0.592 \\
10.5\end{array}$ & $\begin{array}{l}\text { S0 } \\
0.25 \\
0.030 \\
3.0 \\
1.0 \\
1.0 \\
1.0 \\
2.0 \\
0.20 \\
0.0 \\
4.0 \\
1.0 \\
4.00 \\
0.20 \\
0.10 \\
2.1 \\
0.80 \\
0.70 \\
0.80 \\
1.1 \\
2.2 \\
1.1 \\
5.00 \\
0.07 \\
1.00 \\
0.030 \\
0.090 \\
0.030 \\
0.010 \\
0.030 \\
0.030 \\
0.42 \\
0\end{array}$ & $\begin{array}{l}\text { UG/L } \\
\text { UG/L } \\
M G / L \\
U G / L \\
U G / L \\
U G / L \\
U G / L \\
U G / L \\
U G / L \\
U G / L \\
U G / L \\
M G / L \\
\text { UG/L } \\
U G / L \\
U G / L \\
U G / L \\
U G / L \\
U G / L \\
U G / L \\
U G / L \\
U G / L \\
M G / L \\
P C I / L \\
M G / L \\
U G / L \\
U G / L \\
U G / L \\
U G / L \\
U G / L \\
U G / L \\
P C I / L \\
M G / L \\
M G / L \\
U G / L \\
M G / L \\
U G / L \\
U G / L \\
U G / L \\
U G / L \\
U G / L \\
U G / L \\
U G / L \\
U G / L \\
U G / L \\
U G / L \\
U G / L \\
U G / L \\
U G / L \\
U G / L \\
P C I / L \\
U G / L \\
U G / L \\
U G / L \\
U G / L \\
U G / L \\
U G / L \\
U G / L \\
U G / L \\
M G / L \\
U G / L \\
M G / L\end{array}$ & $\begin{array}{l}\text { EPA } 8260 \\
\text { EPA } 353.2 \\
\text { EPA } 300.0 \\
\text { EPA CLP } \\
\text { EPA CLP } \\
\text { EPA CLP } \\
\text { EPA CLP } \\
\text { EPA CLP } \\
\text { EPA CLP } \\
\text { EPA CLP } \\
\text { EPA CLP } \\
\text { EPA } 300.0 \\
\text { EPA } 300.0 \\
\text { EPA CLP } \\
\text { EPA CLP } \\
\text { EPA CLP } \\
\text { EPA CLP } \\
\text { EPA CLP } \\
\text { EPA CLP } \\
\text { EPA CLP } \\
\text { EPA CLP } \\
\text { EPA } 353.1 \\
\text { ASTM } 5174-91 \\
\text { EPA } 300.0 \\
\text { USATHAMA } \\
\text { USATHAMA } \\
\text { USATHAMA } \\
\text { USATHAMA } \\
\text { USATHAMA } \\
\text { USATHAMA } \\
\text { ASTM } 5174-91 \\
\text { EPA } 300.0 \\
\text { EPA } 300.0 \\
\text { EPA } 300.0 \\
\text { EPA } 340.2 \\
\text { EPA CLP } \\
\text { EPA CLP } \\
\text { EPA CLP } \\
\text { EPA CLP } \\
\text { EPA CLP } \\
\text { EPA CLP } \\
\text { EPA CLP } \\
\text { EPA CLP } \\
\text { USATHAMA } \\
\text { USATHAMA } \\
\text { USATHAMA } \\
\text { USATHAMA } \\
\text { USATHAMA } \\
\text { USATHAMA } \\
\text { EPA } 6020 \\
\text { EPA CLP } \\
\text { EPA CLP } \\
\text { EPA CLP } \\
\text { EPA CLP } \\
\text { EPA CLP } \\
\text { EPA CLP } \\
\text { EPA CLP } \\
\text { EPA CLP } \\
\text { EPA } 300.0 \\
\text { EPA } 300.0 \\
\text { EPA } 300.0 \\
\end{array}$ \\
\hline
\end{tabular}




\begin{tabular}{|c|c|c|c|c|c|c|}
\hline ID & DATE_SAM & PARAMETER & CONC & DL & UNITS & METHOO \\
\hline $\begin{array}{l}\text { GW-2040-0497 } \\
G W-2041-0397 \\
G W-2041-0397 \\
G W-2041-0397 \\
G W-2041-0397 \\
G W-2041-0397 \\
G W-2041-0397 \\
G W-2041-0397 \\
G W-2041-0397 \\
G W-2041-0397 \\
G W-2041-0397 \\
G W-2041-0397 \\
G W-2041-0397 \\
G W-2041-0397 \\
G W-2041-0497 \\
G W-2041-0497 \\
G W-2041-0497 \\
G W-2041-0497 \\
G W-2041-0497 \\
G W-2041-0497 \\
G W-2041-0497 \\
G W-2041-0497 \\
G W-2041-0497 \\
G W-2041-0497 \\
G W-2041-0497 \\
G W-2041-0497 \\
G W-2041-0497 \\
G W-2041-0497 \\
G W-2041-0497 \\
G W-2041-0497 \\
G W-2041-0497 \\
G W-2041-0497 \\
G W-2041-0497 \\
G W-2042-0497 \\
G W-2042-0497 \\
G W-2042-0497 \\
G W-2042-0497 \\
G W-2042-0497 \\
G W-2042-0497 \\
G W-2043-0497 \\
G W-2043-0497 \\
G W-2043-0497 \\
G W-2043-0497 \\
G W-2043-0497 \\
G W-2043-0497 \\
G W-2045-0198-A \\
G W-2045-0198-A \\
G W-2045-0198-A \\
G W-2045-0198-A \\
G W-2045-0198-A \\
G W-2045-0198-A \\
G W-2045-0198-A \\
G W-2045-0198-A \\
G W-2045-0198-A \\
G W-2045-0198-A \\
G W-2045-0198-A \\
G W-2045-0198-A \\
G W-2045-0198-A \\
G W-2045-0198-A \\
G W-2045-0198-A \\
G W-2045-0198-A\end{array}$ & $\begin{array}{l}11 / 18 / 97 \\
09 / 17 / 97 \\
09 / 17 / 97 \\
09 / 17 / 97 \\
09 / 17 / 97 \\
09 / 17 / 97 \\
09 / 17 / 97 \\
09 / 17 / 97 \\
09 / 17 / 97 \\
09 / 17 / 97 \\
09 / 17 / 97 \\
09 / 17 / 97 \\
09 / 17 / 97 \\
09 / 17 / 97 \\
11 / 18 / 97 \\
11 / 18 / 97 \\
11 / 18 / 97 \\
11 / 18 / 97 \\
11 / 18 / 97 \\
11 / 18 / 97 \\
11 / 18 / 97 \\
11 / 18 / 97 \\
11 / 18 / 97 \\
111 / 18 / 97 \\
11 / 18 / 97 \\
11 / 18 / 97 \\
11 / 18 / 97 \\
11 / 18 / 97 \\
111 / 18 / 97 \\
111 / 18 / 97 \\
11 / 18 / 97 \\
11 / 18 / 97 \\
11 / 18 / 97 \\
111 / 19 / 97 \\
11 / 19 / 97 \\
11 / 19 / 97 \\
11 / 19 / 97 \\
11 / 19 / 97 \\
111 / 19 / 97 \\
11 / 19 / 97 \\
11 / 19 / 97 \\
11 / 19 / 97 \\
111 / 19 / 97 \\
111 / 19 / 97 \\
11 / 19 / 97 \\
01 / 21 / 98 \\
01 / 21 / 98 \\
011 / 21 / 98 \\
01 / 21 / 98 \\
01 / 21 / 98 \\
01 / 211 / 98 \\
011 / 21 / 98 \\
01 / 21 / 98 \\
01 / 21 / 98 \\
01 / 21 / 98 \\
01 / 21 / 98 \\
011 / 21 / 98 \\
01 / 21 / 98 \\
01 / 21 / 98 \\
01 / 21 / 98 \\
01 / 21 / 98\end{array}$ & $\begin{array}{l}\text { NITRATE-N } \\
\text { URANIUM, TOTAL } \\
\text { CHLORIDE } \\
\text { NITRATE-N } \\
\text { SULFATE } \\
\text { FLUORIDE } \\
\text { ARSENIC } \\
\text { BARIUM } \\
\text { CADMIUM } \\
\text { CHROMIUM } \\
\text { LEAD } \\
\text { SELENIUM } \\
\text { SILVER } \\
\text { MERCURY } \\
\text { NITROBENZENE } \\
1,3-D I N I T R O B E N Z E N E \\
2,4-D I N I T R O T O L U E N E \\
2,6-D I N I T R O T O L U E N E \\
2,4,6-T R I N I T R O T O L U E N E \\
1,3,5-T R I N I T R O B E N Z E N E \\
\text { URANIUM, TOTAL } \\
\text { ARSENIC } \\
\text { BARIUM } \\
\text { CADMIUM } \\
\text { CHROMIUM } \\
\text { LEAD } \\
\text { SELENIUM } \\
\text { SILVER } \\
\text { MERCURY } \\
\text { CHLORIDE } \\
\text { FLUORIDE } \\
\text { SULFATE } \\
\text { NITRATE-N } \\
\text { NITROBENZENE } \\
1,3-D I N I T R O B E N Z E N E \\
2,4-D I N I T R O T O L U E N E \\
2,6-D I N I T R O T O L U E N E \\
2,4,6-T R I N I T R O T O L U E N E \\
1,3,5-T R I N I T R O B E N Z E N E \\
\text { NITROBENZENE } \\
1,3-D I N I T R O B E N Z E N E \\
2,4-D I N I I R O T O L U E N E \\
2,6-D I N I T R O T O L U E N E \\
2,4,6-T R I N I T R O T O L U E N E \\
1,3,5-T R I N I T R O B E N Z E N E \\
\text { CHEMICAL OXYGEN DEMAND } \\
\text { NITRATE-N } \\
\text { NAPHTHALENE } \\
\text { ACENAPHTHYLENE } \\
\text { ACENAPHTHENE } \\
\text { FLUORENE } \\
\text { PHENANTHRENE } \\
\text { ANTHRACENE } \\
\text { FLUORANTHENE } \\
\text { PYRENE } \\
\text { BENZO(A)ANTHRACENE } \\
\text { CHRYSENE } \\
\text { BENZO(B)FLUORANTHENE } \\
\text { BENZO(K)FLUORANTHENE } \\
\text { BENZO(A)PYRENE } \\
\text { DIBENZO(A,H)ANTHRACENE }\end{array}$ & 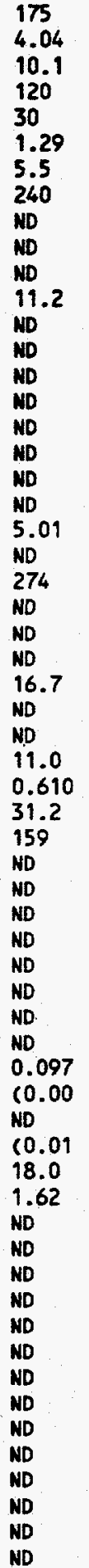 & $\begin{array}{l}20.0 \\
0.42 \\
0.020 \\
0.20 \\
0.10 \\
0.25 \\
3.0 \\
1.0 \\
1.0 \\
1.0 \\
2.0 \\
4.0 \\
1.0 \\
0.0 \\
0.2 \\
0.030 \\
0.090 \\
0.030 \\
0.010 \\
0.030 \\
0.030 \\
0.07 \\
3\end{array}$ & 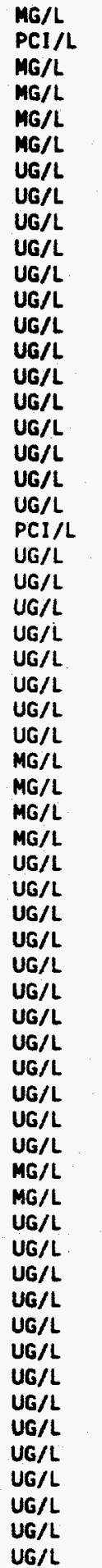 & $\begin{array}{l}\text { EPA } 353.2 \\
\text { ASTM } 5174-91 \\
\text { EPA } 300.0 \\
\text { EPA } 300.0 \\
\text { EPA } 300.0 \\
\text { EPA } 340.2 \\
\text { EPA CLP } \\
\text { EPA CLP } \\
\text { EPA CLP } \\
\text { EPA CLP } \\
\text { EPA CLP } \\
\text { EPA CLP } \\
\text { EPA CLP } \\
\text { EPA CLP } \\
\text { USATHAMA } \\
\text { USATHAMA } \\
\text { USATHAMA } \\
\text { USATHAMA } \\
\text { USATHAMA } \\
\text { USATHAMA } \\
\text { EPA } 6020 \\
\text { EPA CLP } \\
\text { EPA CLP } \\
\text { EPA CLP } \\
\text { EPA CLP } \\
\text { EPA CLP } \\
\text { EPA CLP } \\
\text { EPA CLP } \\
\text { EPA CLP } \\
\text { EPA } 300.0 \\
\text { EPA } 300.0 \\
\text { EPA } 300.0 \\
\text { EPA } 353.2 \\
\text { USATHAMA } \\
\text { USATHAMA } \\
\text { USATHAMA } \\
\text { USATHAMA } \\
\text { USATHAMA } \\
\text { USATHAMA } \\
\text { USATHAMA } \\
\text { USATHAMA } \\
\text { USATHAMA } \\
\text { USATHAMA } \\
\text { USATHAMA } \\
\text { USATHAMA } \\
\text { EPA } 410.4 \\
\text { EPA } 353.1 \\
\text { EPA } 8310 \\
\text { EPA } 8310 \\
\text { EPA } 8310 \\
\text { EPA } 8310 \\
\text { EPA } 8310 \\
\text { EPA } 8310 \\
\text { EPA } 8310 \\
\text { EPA } 8310 \\
\text { EPA } 8310 \\
\text { EPA } 8310 \\
\text { EPA } 8310 \\
\text { EPA } 8310 \\
\text { EPA } 8310 \\
\text { EPA } 8310 \\
\end{array}$ \\
\hline
\end{tabular}




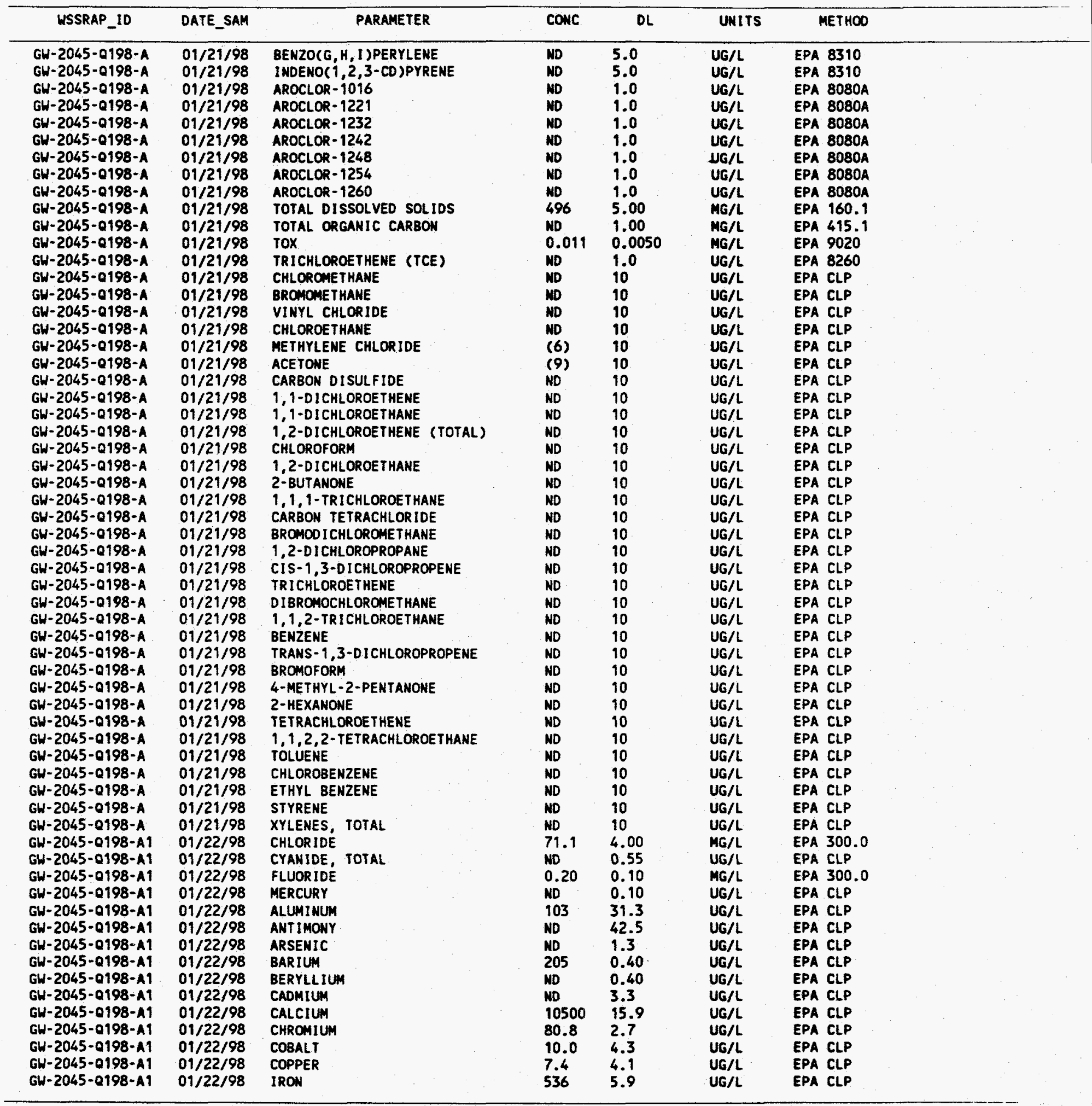




\begin{tabular}{|c|c|c|c|c|c|c|}
\hline WSSRAP_10 & DATE_SAM & PARAMETER & CONC & $\mathrm{DL}$ & UNITS & METHOD \\
\hline 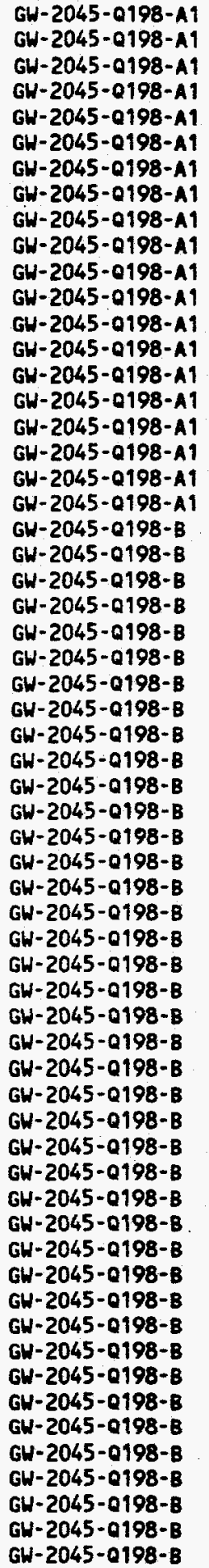 & $\begin{array}{l}01 / 22 / 98 \\
01 / 22 / 98 \\
01 / 22 / 98 \\
01 / 22 / 98 \\
01 / 22 / 98 \\
01 / 22 / 98 \\
01 / 22 / 98 \\
01 / 22 / 98 \\
01 / 22 / 98 \\
01 / 22 / 98 \\
01 / 22 / 98 \\
01 / 22 / 98 \\
01 / 22 / 98 \\
01 / 22 / 98 \\
01 / 22 / 98 \\
01 / 22 / 98 \\
01 / 22 / 98 \\
01 / 22 / 98 \\
01 / 22 / 98 \\
01 / 22 / 98 \\
01 / 23 / 98 \\
01 / 23 / 98 \\
01 / 23 / 98 \\
01 / 23 / 98 \\
01 / 23 / 98 \\
01 / 23 / 98 \\
01 / 23 / 98 \\
01 / 23 / 98 \\
01 / 23 / 98 \\
01 / 23 / 98 \\
01 / 23 / 98 \\
01 / 23 / 98 \\
01 / 23 / 98 \\
01 / 23 / 98 \\
01 / 23 / 98 \\
01 / 23 / 98 \\
01 / 23 / 98 \\
01 / 23 / 98 \\
01 / 23 / 98 \\
01 / 23 / 98 \\
01 / 23 / 98 \\
01 / 23 / 98 \\
01 / 23 / 98 \\
01 / 23 / 98 \\
01 / 23 / 98 \\
01 / 23 / 98 \\
01 / 23 / 98 \\
01 / 23 / 98 \\
01 / 23 / 98 \\
01 / 23 / 98 \\
01 / 23 / 98 \\
01 / 23 / 98 \\
01 / 23 / 98 \\
01 / 23 / 98 \\
01 / 23 / 98 \\
01 / 23 / 98 \\
01 / 23 / 98 \\
01 / 23 / 98 \\
01 / 23 / 98 \\
01 / 23 / 98 \\
01 / 23 / 98\end{array}$ & 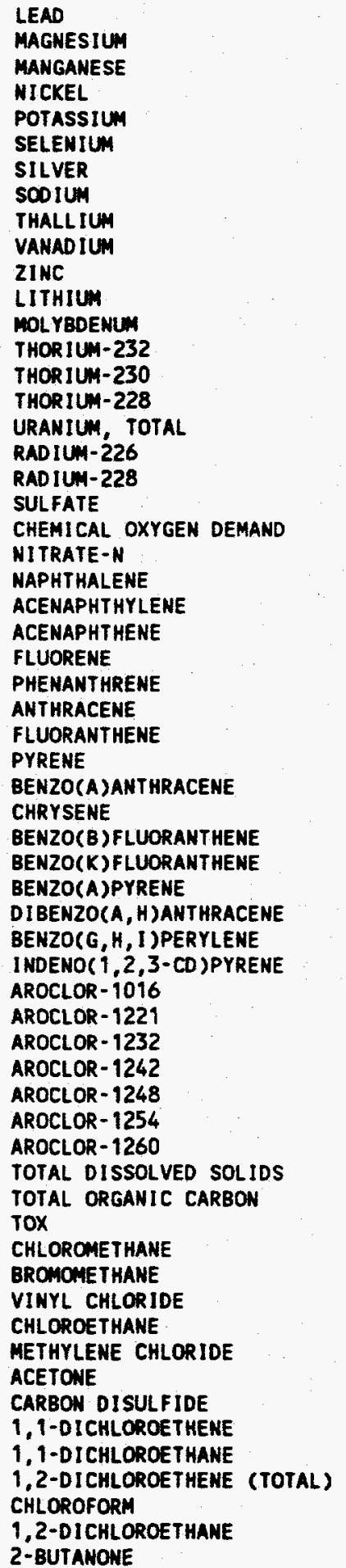 & $\begin{array}{l}\text { ND } \\
45500 \\
129 \\
771 \\
2200 \\
\text { ND } \\
\text { ND } \\
18200 \\
\text { ND } \\
11.0 \\
19.3 \\
\text { ND } \\
19.2 \\
\text { ND } \\
\text { CO.06 } \\
\text { CO.06 } \\
\text { ND } \\
O .236 \\
\text { CO.07 } \\
\text { 30.0 } \\
14.0 \\
1.63 \\
\text { ND } \\
\text { ND } \\
\text { ND } \\
\text { ND } \\
\text { ND } \\
\text { ND } \\
\text { ND } \\
\text { ND } \\
\text { ND } \\
\text { ND } \\
\text { ND } \\
\text { ND } \\
\text { ND } \\
\text { ND } \\
\text { ND } \\
\text { ND } \\
\text { ND } \\
\text { ND } \\
\text { ND } \\
\text { ND } \\
\text { ND } \\
\text { ND } \\
\text { ND } \\
497 \\
1.02 \\
O .039 \\
\text { ND } \\
\text { ND } \\
\text { ND } \\
\text { ND } \\
170 \\
17 \\
\text { ND } \\
\text { ND } \\
\text { ND } \\
\text { ND } \\
\text { ND } \\
\text { ND } \\
\text { ND }\end{array}$ & 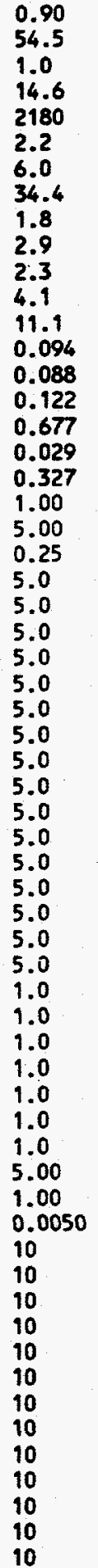 & 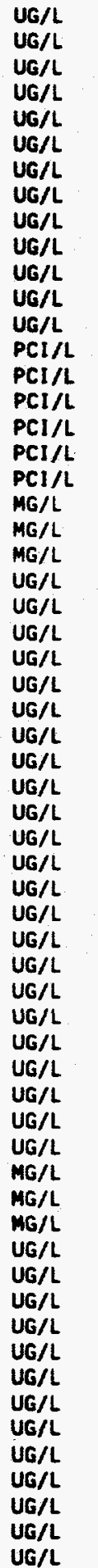 & $\begin{array}{l}\text { EPA CLP } \\
\text { EPA CLP } \\
\text { EPA CLP } \\
\text { EPA CLP } \\
\text { EPA CLP } \\
\text { EPA CLP } \\
\text { EPA CLP } \\
\text { EPA CLP } \\
\text { EPA CLP } \\
\text { EPA CLP } \\
\text { EPA CLP } \\
\text { EPA CLP } \\
\text { EPA CLP } \\
\text { NAS-NS-3004 } \\
\text { NAS-NS-3004 } \\
\text { NAS-NS-3004 } \\
\text { ASTM } 5174-91 \\
\text { EPA } 904.0 \\
\text { EPA } 904.0 \\
\text { EPA } 300.0 \\
\text { EPA } 410.4 \\
\text { EPA } 353.1 \\
\text { EPA } 8310 \\
\text { EPA } 8310 \\
\text { EPA } 8310 \\
\text { EPA } 8310 \\
\text { EPA } 8310 \\
\text { EPA } 8310 \\
\text { EPA } 8310 \\
\text { EPA } 8310 \\
\text { EPA } 8310 \\
\text { EPA } 8310 \\
\text { EPA } 8310 \\
\text { EPA } 8310 \\
\text { EPA } 8310 \\
\text { EPA } 8310 \\
\text { EPA } 8310 \\
\text { EPA } 8310 \\
\text { EPA } 8080 A \\
\text { EPA } 8080 A \\
\text { EPA } 8080 A \\
\text { EPA } 8080 A \\
\text { EPA } 8080 A \\
\text { EPA } 8080 A \\
\text { EPA } 8080 A \\
\text { EPA } 160.1 \\
\text { EPA } 415.1 \\
\text { EPA } 9020 \\
\text { EPA CLP } \\
\text { EPA CLP } \\
\text { EPA CLP } \\
\text { EPA CLP } \\
\text { EPA CLP } \\
\text { EPA CLP } \\
\text { EPA CLP } \\
\text { EPA CLP } \\
\text { EPA CLP } \\
\text { EPA CLP } \\
\text { EPA CLP } \\
\text { EPA CLP } \\
\text { EPA CLP }\end{array}$ \\
\hline
\end{tabular}




\begin{tabular}{|c|c|c|c|c|c|c|}
\hline WSSRAP_ID & DATE_SAM & PARAMETER & CONC & $\mathrm{DL}$ & UNITS & METHOD \\
\hline $\begin{array}{l}G W-2045-0198-B \\
G W-2045-0198-B \\
G W-2045-0198-B \\
G W-2045-0198-8 \\
G W-2045-0198-B \\
G W-2045-0198-B \\
G W-2045-0198-B \\
G W-2045-0198-B \\
G W-2045-0198-B \\
G W-2045-0198-B \\
G W-2045-0198-8 \\
G W-2045-0198-B \\
G W-2045-0198-B \\
G W-2045-0199-B \\
G W-2045-0198-B \\
G W-2045-0198-B \\
G W-2045-0198-B \\
G W-2045-0198-B \\
G W-2045-0198-B \\
G W-2045-0198-B \\
G W-2045-0198-B 1 \\
G W-2045-0198-B 1 \\
G W-2045-0198-81 \\
G W-2045-0198-81 \\
G W-2045-0198-B 1 \\
G W-2045-0198-B 1 \\
G W-2045-0198-B 1 \\
G W-2045-0198-B 1 \\
G W-2045-0198-B 1 \\
G W-2045-0198-B 1 \\
G W-2045-0198-B 1 \\
G W-2045-0198-B 1 \\
G W-2045-0198-B 1 \\
G W-2045-0198-B 1 \\
G W-2045-0198-B 1 \\
G W-2045-0198-B 1 \\
G W-2045-0198-B 1 \\
G W-2045-0198-B 1 \\
G W-2045-0198-81 \\
G W-2045-0198-B 1 \\
G W-2045-0198-B 1 \\
G W-2045-0198-B 1 \\
G W-2045-0198-B 1 \\
G W-2045-0198-B 1 \\
G W-2045-0198-B 1 \\
G W-2045-0198-B 1 \\
G W-2045-0198-B 1 \\
G W-2045-0198-B 1 \\
G W-2045-0198-B 1 \\
G W-2045-0198-81 \\
G W-2045-0198-B 1 \\
G W-2045-0198-B 1 \\
G W-2045-0198-B 1 \\
G W-2045-0198-B 1 \\
G W-2045-0198-81 \\
G W-2045-0198-C 1 \\
G W-2045-0198-C 1 \\
G W-2045-0198-C 1 \\
G W-2045-0198-C 1 \\
G W-2045-0198-C 1 \\
G W-2045-0198-C 1\end{array}$ & $\begin{array}{l}01 / 23 / 98 \\
01 / 23 / 98 \\
01 / 23 / 98 \\
01 / 23 / 98 \\
01 / 23 / 98 \\
01 / 23 / 98 \\
01 / 23 / 98 \\
01 / 23 / 98 \\
01 / 23 / 98 \\
01 / 23 / 98 \\
01 / 23 / 98 \\
01 / 23 / 98 \\
01 / 23 / 98 \\
01 / 23 / 98 \\
01 / 23 / 98 \\
01 / 23 / 98 \\
01 / 23 / 98 \\
01 / 23 / 98 \\
01 / 23 / 98 \\
01 / 23 / 98 \\
01 / 26 / 98 \\
01 / 26 / 98 \\
01 / 26 / 98 \\
01 / 26 / 98 \\
01 / 26 / 98 \\
01 / 26 / 98 \\
01 / 26 / 98 \\
01 / 26 / 98 \\
01 / 26 / 98 \\
01 / 26 / 98 \\
01 / 26 / 98 \\
01 / 26 / 98 \\
01 / 26 / 98 \\
01 / 26 / 98 \\
01 / 26 / 98 \\
01 / 26 / 98 \\
01 / 26 / 98 \\
01 / 26 / 98 \\
01 / 26 / 98 \\
01 / 26 / 98 \\
01 / 26 / 98 \\
01 / 26 / 98 \\
01 / 26 / 98 \\
01 / 26 / 98 \\
01 / 26 / 98 \\
01 / 26 / 98 \\
01 / 26 / 98 \\
01 / 26 / 98 \\
01 / 26 / 98 \\
01 / 26 / 98 \\
01 / 26 / 98 \\
01 / 26 / 98 \\
01 / 26 / 98 \\
01 / 26 / 98 \\
01 / 26 / 98 \\
01 / 28 / 98 \\
01 / 28 / 98 \\
01 / 28 / 98 \\
01 / 28 / 98 \\
01 / 28 / 98 \\
01 / 28 / 98\end{array}$ & 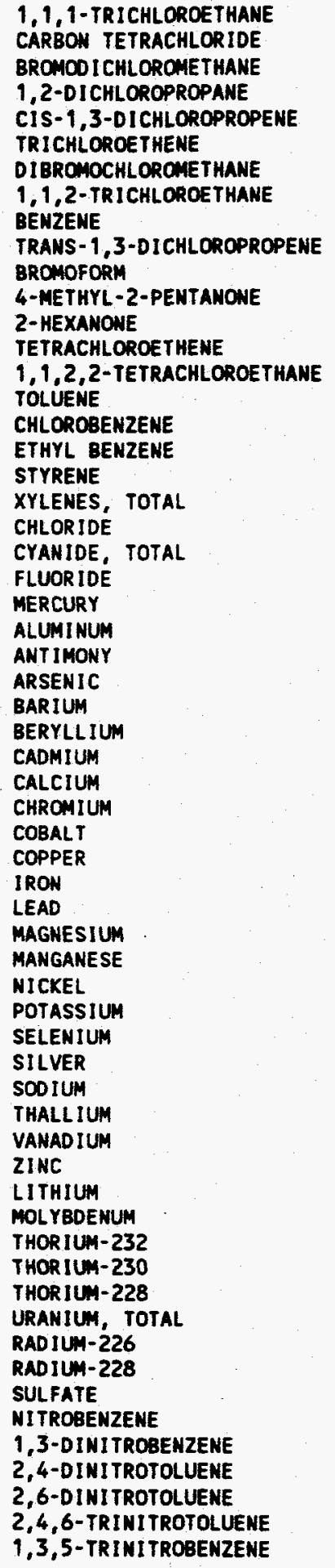 & $\begin{array}{l}\text { ND } \\
\text { ND } \\
\text { ND } \\
\text { ND } \\
\text { ND } \\
\text { ND } \\
\text { ND } \\
\text { ND } \\
\text { ND } \\
\text { ND } \\
\text { ND } \\
\text { ND } \\
\text { ND } \\
\text { ND } \\
\text { ND } \\
\text { MD } \\
\text { ND } \\
\text { ND } \\
\text { ND } \\
\text { ND } \\
65.8 \\
0.62 \\
0.23 \\
\text { ND } \\
55.1 \\
\text { ND } \\
\text { ND } \\
193 \\
0.54 \\
\text { ND } \\
99800 \\
33.0 \\
4.3 \\
\text { ND } \\
180 \\
\text { ND } \\
44000 \\
53.5 \\
328 \\
2660 \\
\text { ND } \\
\text { ND } \\
16600 \\
\text { ND } \\
17.3 \\
12.6 \\
19.9 \\
\text { ND } \\
0.038 \\
0.561 \\
\text { Co.04 } \\
\text { ND } \\
0.277 \\
\text { C0.26 } \\
25.4 \\
\text { ND } \\
0.17 \\
0.15 \\
0.74 \\
\text { ND } \\
0.030\end{array}$ & $\begin{array}{l}10 \\
10 \\
10 \\
10 \\
10 \\
10 \\
10 \\
10 \\
10 \\
10 \\
10 \\
10 \\
10 \\
10 \\
10 \\
10 \\
10 \\
10 \\
10 \\
10 \\
4.00 \\
0.55 \\
0.10 \\
0.10 \\
31.3 \\
42.5 \\
1.3 \\
0.40 \\
0.40 \\
3.3 \\
15.9 \\
2.7 \\
4.3 \\
4.1 \\
5.9 \\
0.90 \\
54.5 \\
1.0 \\
14.6 \\
2180 \\
2.2 \\
6.0 \\
34.4 \\
1.8 \\
2.9 \\
2.3 \\
4.1 \\
11.1 \\
0.026 \\
0.080 \\
0.111 \\
0.677 \\
0.129 \\
0.273 \\
1.00 \\
0.030 \\
0.090 \\
0.030 \\
0.010 \\
0.030 \\
0.030\end{array}$ & 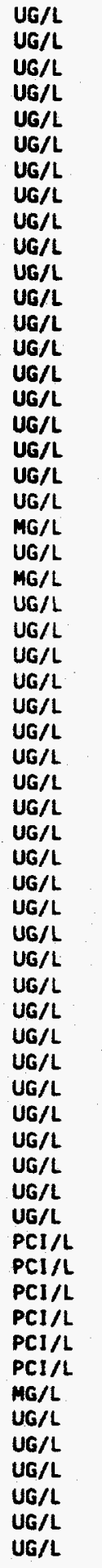 & $\begin{array}{l}\text { EPA CLP } \\
\text { EPA CLP } \\
\text { EPA CLP } \\
\text { EPA CLP } \\
\text { EPA CLP } \\
\text { EPA CLP } \\
\text { EPA CLP } \\
\text { EPA CLP } \\
\text { EPA CLP } \\
\text { EPA CLP } \\
\text { EPA CLP } \\
\text { EPA CLP } \\
\text { EPA CLP } \\
\text { EPA CLP } \\
\text { EPA CLP } \\
\text { EPA CLP } \\
\text { EPA CLP } \\
\text { EPA CLP } \\
\text { EPA CLP } \\
\text { EPA CLP } \\
\text { EPA } 300.0 \\
\text { EPA CLP } \\
\text { EPA } 300.0 \\
\text { EPA CLP } \\
\text { EPA CLP } \\
\text { EPA CLP } \\
\text { EPA CLP } \\
\text { EPA CLP } \\
\text { EPA CLP } \\
\text { EPA CLP } \\
\text { EPA CLP } \\
\text { EPA CLP } \\
\text { EPA CLP } \\
\text { EPA CLP } \\
\text { EPA CLP } \\
\text { EPA CLP } \\
\text { EPA CLP } \\
\text { EPA CLP } \\
\text { EPA CLP } \\
\text { EPA CLP } \\
\text { EPA CLP } \\
\text { EPA CLP } \\
\text { EPA CLP } \\
\text { EPA CLP } \\
\text { EPA CLP } \\
\text { EPA CLP } \\
\text { EPA CLP } \\
\text { EPA CLP } \\
\text { NAS-NS-3004 } \\
\text { NAS-NS-3004 } \\
\text { NAS-HS-3004 } \\
\text { ASTM } 5174-91 \\
\text { EPA } 904.0 \\
\text { EPA } 904.0 \\
\text { EPA } 300.0 \\
\text { USATHAMA } \\
\text { USATHAMA } \\
\text { USATHAMA } \\
\text { USATHAMA } \\
\text { USATHAMA } \\
\text { USATHAMA } \\
\end{array}$ \\
\hline
\end{tabular}




\begin{tabular}{|c|c|c|c|c|c|c|}
\hline USSRAP_ID & DATE_SAM & PARAMETER & CONC & $D L$ & UNITS & METHOO \\
\hline 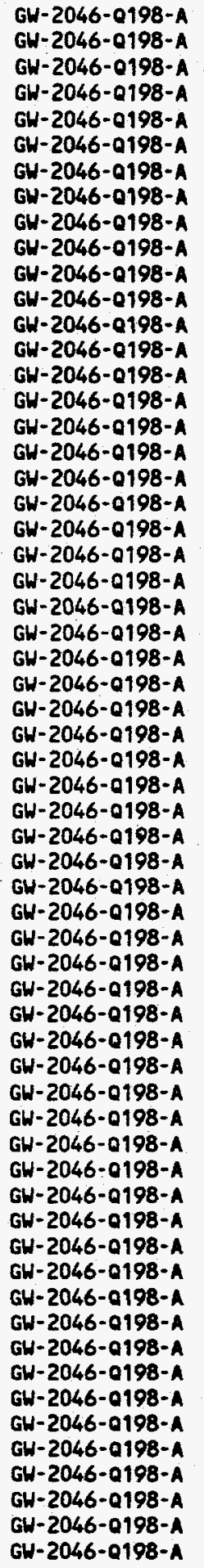 & $\begin{array}{l}01 / 21 / 98 \\
01 / 21 / 98 \\
01 / 21 / 98 \\
01 / 21 / 98 \\
01 / 21 / 98 \\
01 / 21 / 98 \\
01 / 21 / 98 \\
01 / 21 / 98 \\
01 / 21 / 98 \\
01 / 21 / 98 \\
01 / 21 / 98 \\
01 / 21 / 98 \\
01 / 21 / 98 \\
01 / 21 / 98 \\
01 / 21 / 98 \\
01 / 21 / 98 \\
01 / 21 / 98 \\
01 / 21 / 98 \\
01 / 21 / 98 \\
01 / 21 / 98 \\
01 / 21 / 98 \\
01 / 21 / 98 \\
01 / 21 / 98 \\
01 / 21 / 98 \\
01 / 21 / 98 \\
01 / 21 / 98 \\
01 / 21 / 98 \\
01121 / 98 \\
01 / 21 / 98 \\
01 / 21 / 98 \\
01 / 21 / 98 \\
01 / 21 / 98 \\
01 / 21 / 98 \\
011 / 21 / 98 \\
01 / 21 / 98 \\
01 / 21 / 98 \\
01 / 21 / 98 \\
01 / 21 / 98 \\
011 / 21 / 98 \\
01 / 21 / 98 \\
01 / 21 / 98 \\
01 / 21 / 98 \\
01 / 21 / 98 \\
01 / 21 / 98 \\
01 / 21 / 98 \\
01 / 21 / 98 \\
01 / 21 / 98 \\
01 / 21 / 98 \\
01 / 21 / 98 \\
01 / 21 / 98 \\
01 / 21 / 98 \\
01121 / 98 \\
011 / 21 / 98 \\
01 / 21 / 98 \\
01 / 21 / 98 \\
01 / 211 / 98 \\
01121 / 98 \\
01 / 21 / 98 \\
01 / 21 / 98 \\
01 / 21 / 98 \\
01 / 211 / 98\end{array}$ & 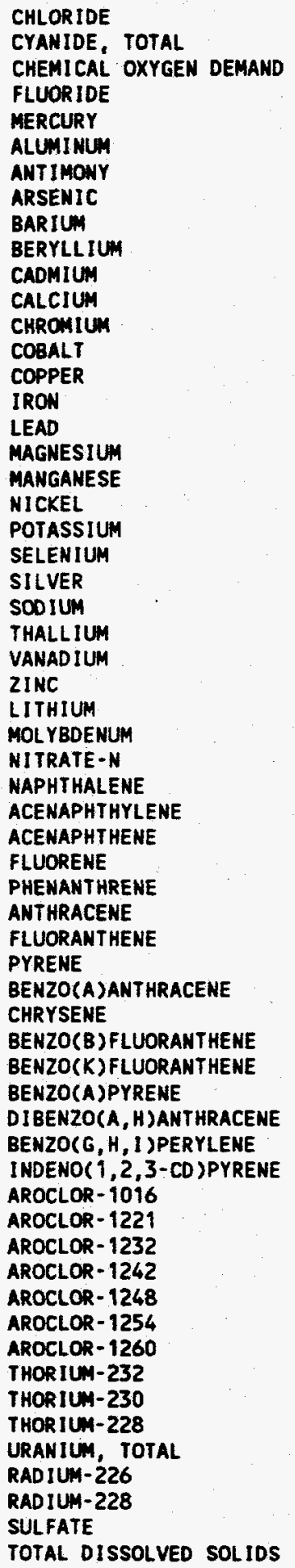 & 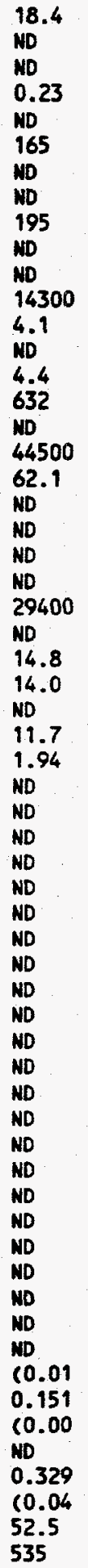 & $\begin{array}{l}1.00 \\
0.55 \\
5.00 \\
0.10 \\
0.10 \\
31.3 \\
42.5 \\
1.3 \\
0.40 \\
0.40 \\
3.3 \\
15.9 \\
2.7 \\
4.3 \\
4.1 \\
5.9 \\
0.90 \\
54.5 \\
1.0 \\
14.6 \\
2180 \\
2.2 \\
6.0 \\
34.4 \\
1.8 \\
2.9 \\
2.3 \\
4.1 \\
11.1 \\
0.25 \\
5.0 \\
5.0 \\
5.0 \\
5.0 \\
5.0 \\
5.0 \\
5.0 \\
5.0 \\
5.0 \\
5.0 \\
5.0 \\
5.0 \\
5.0 \\
5.0 \\
5.0 \\
5.0 \\
1.0 \\
1.0 \\
1.0 \\
1.0 \\
1.0 \\
1.0 \\
1.0 \\
0.072 \\
0.029 \\
0.164 \\
0.677 \\
0.029 \\
0.317 \\
2.50 \\
5.00 \\
\end{array}$ & $\begin{array}{l}\text { MG/L } \\
U G / L \\
M G / L \\
M G / L \\
U G / L \\
U G / L \\
U G / L \\
U G / L \\
U G / L \\
U G / L \\
U G / L \\
U G / L \\
U G / L \\
U G / L \\
U G / L \\
U G / L \\
U G / L \\
U G / L \\
U G / L \\
U G / L \\
U G / L \\
U G / L \\
U G / L \\
U G / L \\
U G / L \\
U G / L \\
U G / L \\
U G / L \\
U G / L \\
M G / L \\
U G / L \\
U G / L \\
U G / L \\
U G / L \\
U G / L \\
U G / L \\
U G / L \\
U G / L \\
U G / L \\
U G / L \\
U G / L \\
U G / L \\
U G / L \\
U G / L \\
U G / L \\
U G / L \\
U G / L \\
U G / L \\
U G / L \\
U G / L \\
U G / L \\
U G / L \\
U G / L \\
P C I / L \\
P C I / L \\
P C I / L \\
P C I / L \\
P C I / L \\
P C I / L \\
M G / L \\
M G / L \\
\end{array}$ & $\begin{array}{l}\text { EPA } 300.0 \\
\text { EPA CLP } \\
\text { EPA } 410.4 \\
\text { EPA } 300.0 \\
\text { EPA CLP } \\
\text { EPA CLP } \\
\text { EPA CLP } \\
\text { EPA CLP } \\
\text { EPA CLP } \\
\text { EPA CLP } \\
\text { EPA CLP } \\
\text { EPA CLP } \\
\text { EPA CLP } \\
\text { EPA CLP } \\
\text { EPA CLP } \\
\text { EPA CLP } \\
\text { EPA CLP } \\
\text { EPA CLP } \\
\text { EPA CLP } \\
\text { EPA CLP } \\
\text { EPA CLP } \\
\text { EPA CLP } \\
\text { EPA CLP } \\
\text { EPA CLP } \\
\text { EPA CLP } \\
\text { EPA CLP } \\
\text { EPA CLP } \\
\text { EPA CLP } \\
\text { EPA CLP } \\
\text { EPA } 353.1 \\
\text { EPA } 8310 \\
\text { EPA } 8310 \\
\text { EPA } 8310 \\
\text { EPA } 8310 \\
\text { EPA } 8310 \\
\text { EPA } 8310 \\
\text { EPA } 8310 \\
\text { EPA } 8310 \\
\text { EPA } 8310 \\
\text { EPA } 8310 \\
\text { EPA } 8310 \\
\text { EPA } 8310 \\
\text { EPA } 8310 \\
\text { EPA } 8310 \\
\text { EPA } 8310 \\
\text { EPA } 8310 \\
\text { EPA } 8080 A \\
\text { EPA } 8080 A \\
\text { EPA } 8080 A \\
\text { EPA } 8080 A \\
\text { EPA } 8080 A \\
\text { EPA } 8080 A \\
\text { EPA } 8080 A \\
\text { NAS-NS-3004 } \\
\text { NAS-NS-3004 } \\
\text { NAS-NS-3004 } \\
\text { ASTM } 5174-91 \\
\text { EPA } 904.0 \\
\text { EPA } 904.0 \\
\text { EPA } 300.0 \\
\text { EPA } 160.1 \\
\end{array}$ \\
\hline
\end{tabular}




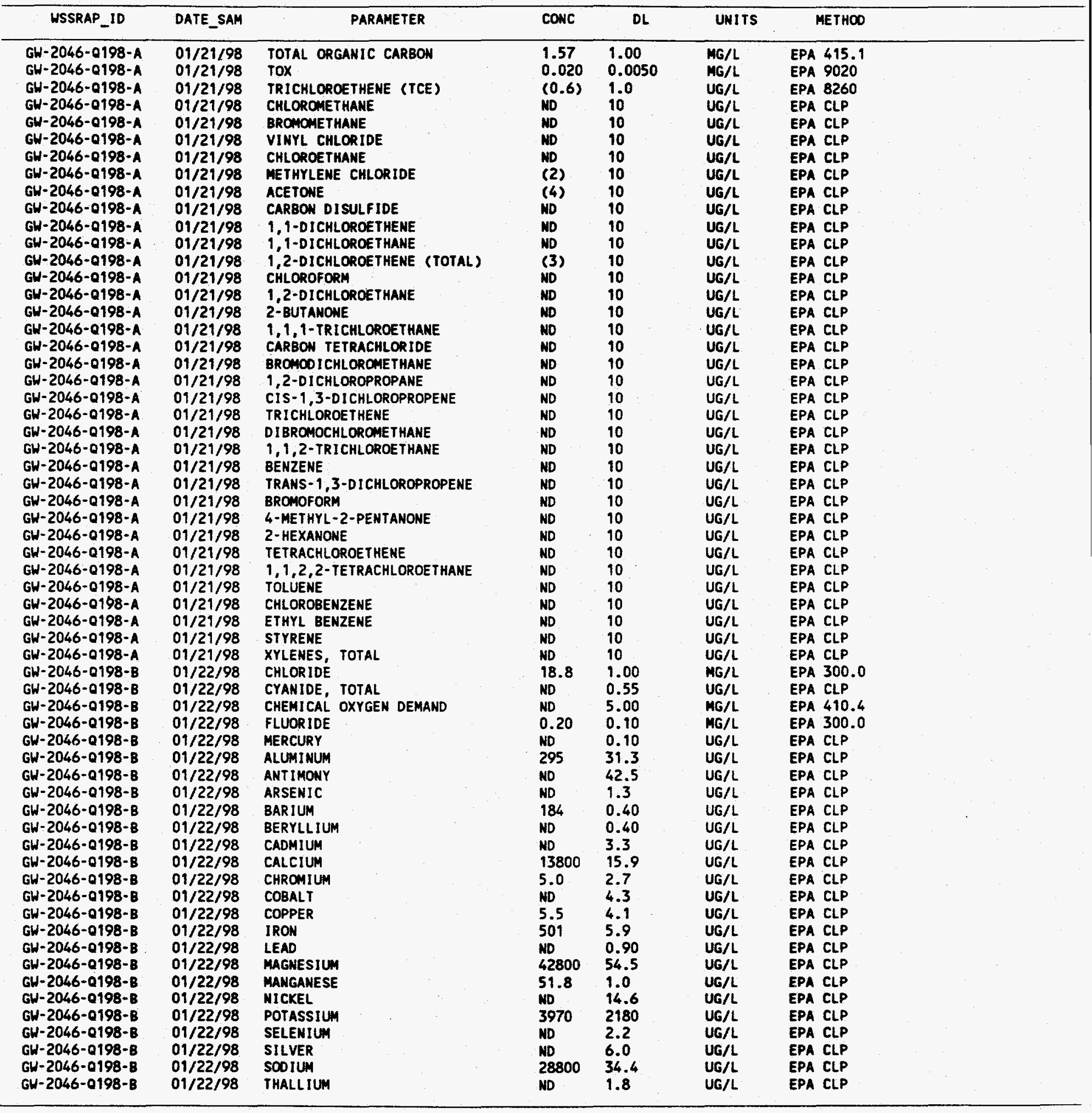




\begin{tabular}{|c|c|c|c|c|c|c|}
\hline USSRAP_ID & DATE_SAM & PARAMETER & CONC & DL & UNITS & METHOO \\
\hline 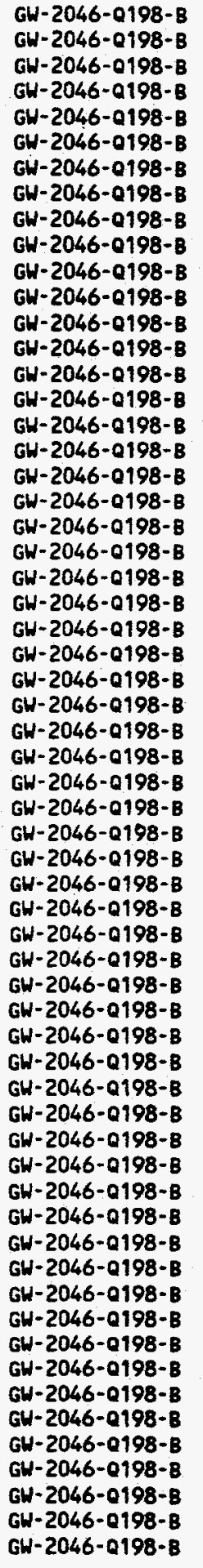 & $\begin{array}{l}01 / 22 / 98 \\
01 / 22 / 98 \\
01 / 22 / 98 \\
01 / 22 / 98 \\
01 / 22 / 98 \\
01 / 22 / 98 \\
01 / 22 / 98 \\
01 / 22 / 98 \\
01 / 22 / 98 \\
01 / 22 / 98 \\
01 / 22 / 98 \\
01 / 22 / 98 \\
01 / 22 / 98 \\
01 / 22 / 98 \\
01 / 22 / 98 \\
01 / 22 / 98 \\
01 / 22 / 98 \\
01 / 22 / 98 \\
01 / 22 / 98 \\
01 / 22 / 98 \\
01 / 22 / 98 \\
01 / 22 / 98 \\
01 / 22 / 98 \\
01 / 22 / 98 \\
01 / 22 / 98 \\
01 / 22 / 98 \\
01 / 22 / 98 \\
01 / 22 / 98 \\
01 / 22 / 98 \\
01 / 22 / 98 \\
01 / 22 / 98 \\
01 / 22 / 98 \\
01 / 22 / 98 \\
01 / 22 / 98 \\
01 / 22 / 98 \\
01 / 22 / 98 \\
01 / 22 / 98 \\
01 / 22 / 98 \\
01 / 22 / 98 \\
01 / 22 / 98 \\
01 / 22 / 98 \\
01 / 22 / 98 \\
01 / 22 / 98 \\
01 / 22 / 98 \\
01 / 22 / 98 \\
01 / 22 / 98 \\
01 / 22 / 98 \\
01 / 22 / 98 \\
011 / 22 / 98 \\
01 / 22 / 98 \\
01 / 22 / 98 \\
01 / 22 / 98 \\
01 / 22 / 98 \\
01 / 22 / 98 \\
01 / 22 / 98 \\
01 / 22 / 98 \\
01 / 22 / 98 \\
01 / 22 / 98 \\
01 / 22 / 98 \\
01 / 22 / 98 \\
01 / 22 / 98\end{array}$ & 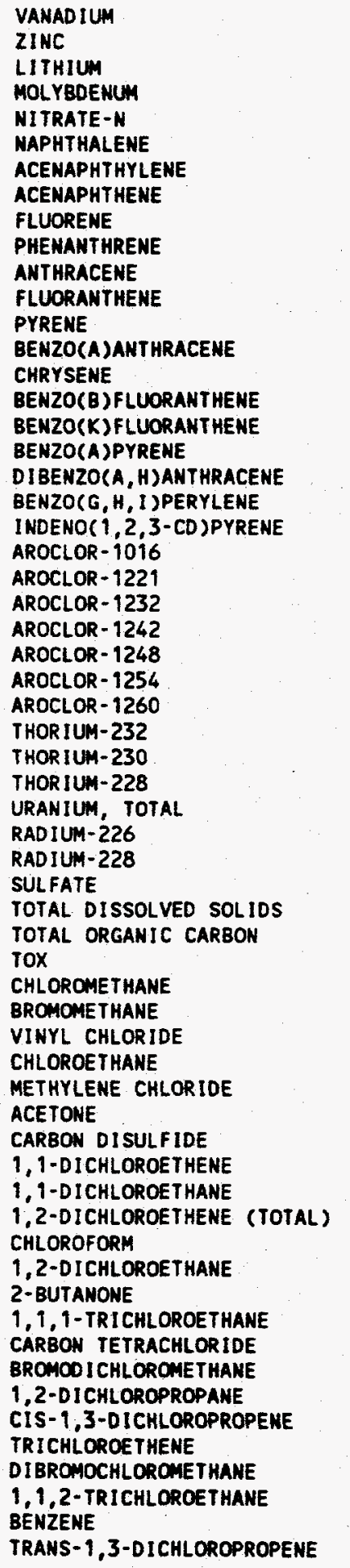 & 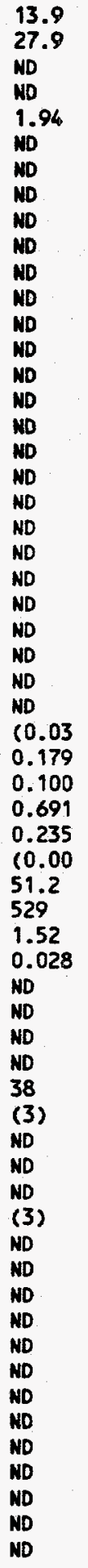 & 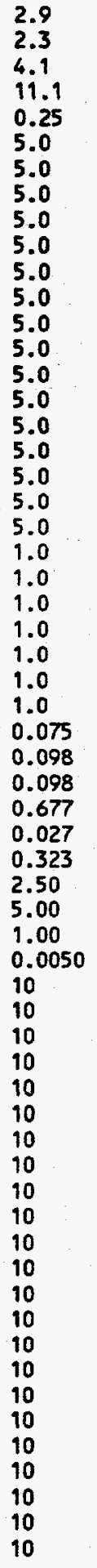 & 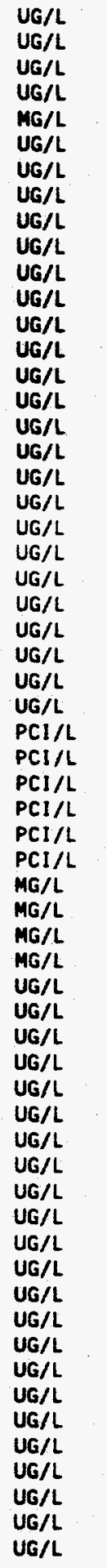 & $\begin{array}{l}\text { EPA CLP } \\
\text { EPA CLP } \\
\text { EPA CLP } \\
\text { EPA CLP } \\
\text { EPA } 353.1 \\
\text { EPA } 8310 \\
\text { EPA } 8310 \\
\text { EPA } 8310 \\
\text { EPA } 8310 \\
\text { EPA } 8310 \\
\text { EPA } 8310 \\
\text { EPA } 8310 \\
\text { EPA } 8310 \\
\text { EPA } 8310 \\
\text { EPA } 8310 \\
\text { EPA } 8310 \\
\text { EPA } 8310 \\
\text { EPA } 8310 \\
\text { EPA } 8310 \\
\text { EPA } 8310 \\
\text { EPA } 8310 \\
\text { EPA } 8080 A \\
\text { EPA } 8080 A \\
\text { EPA } 8080 A \\
\text { EPA } 8080 A \\
\text { EPA } 8080 A \\
\text { EPA } 8080 A \\
\text { EPA } 8080 A \\
\text { NAS- } 15-3004 \\
\text { NAS-NS-3004 } \\
\text { NAS-NS-3004 } \\
\text { ASTM } 5174-91 \\
\text { EPA } 904.0 \\
\text { EPA } 904.0 \\
\text { EPA } 300.0 \\
\text { EPA } 160.1 \\
\text { EPA } 415.1 \\
\text { EPA } 9020 \\
\text { EPA CLP } \\
\text { EPA CLP } \\
\text { EPA CLP } \\
\text { EPA CLP } \\
\text { EPA CLP } \\
\text { EPA CLP } \\
\text { EPA CLP } \\
\text { EPA CLP } \\
\text { EPA CLP } \\
\text { EPA CLP } \\
\text { EPA CLP } \\
\text { EPA CLP } \\
\text { EPA CLP } \\
\text { EPA CLP } \\
\text { EPA CLP } \\
\text { EPA CLP } \\
\text { EPA CLP } \\
\text { EPA CLP } \\
\text { EPA CLP } \\
\text { EPA CLP } \\
\text { EPA CLP } \\
\text { EPA CLP } \\
\text { EPA CLP } \\
\end{array}$ \\
\hline
\end{tabular}




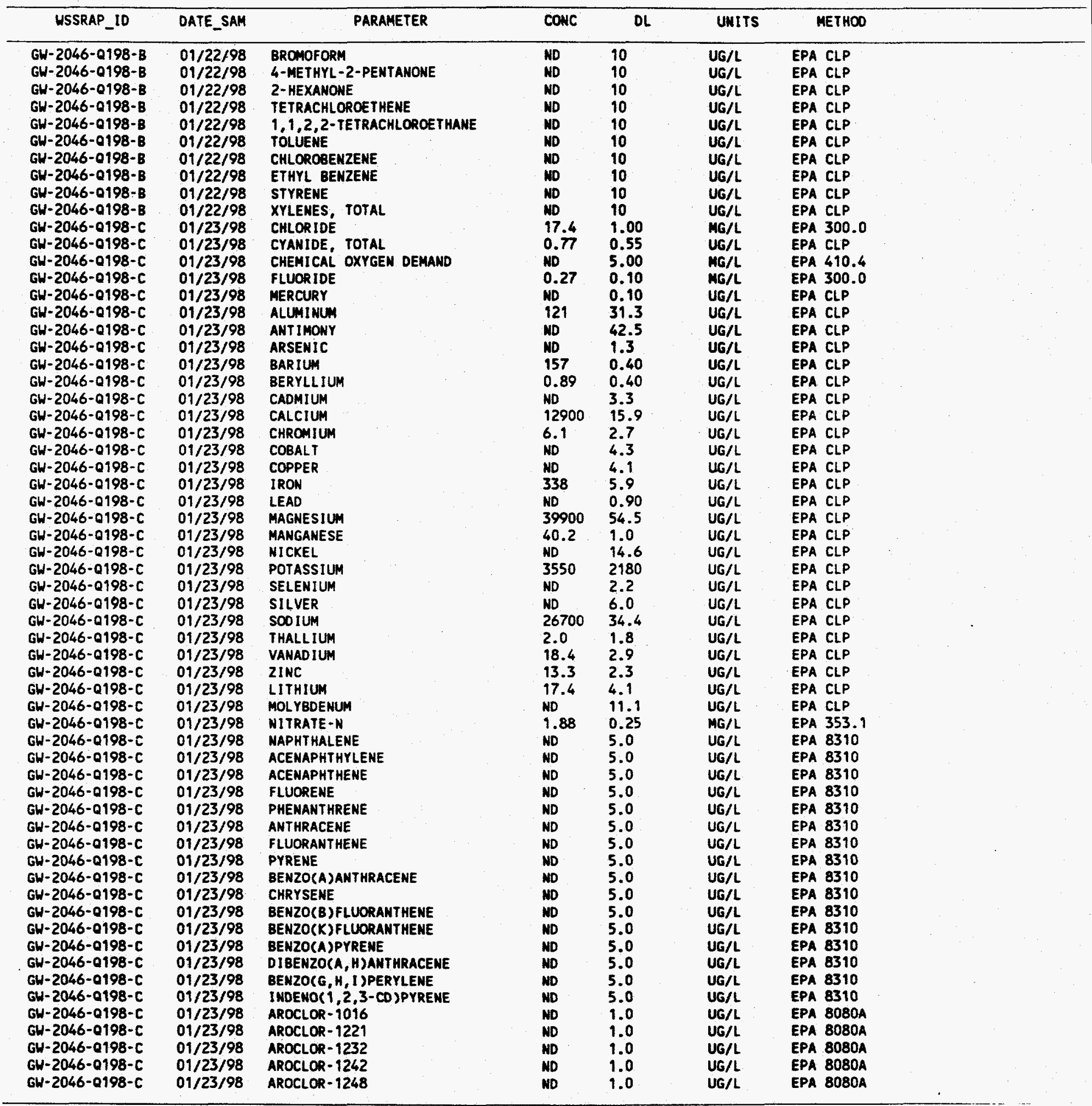




\begin{tabular}{|c|c|c|c|c|c|c|}
\hline WSSRAP_ID & DATE_SAM & PARAMETER & CONC & DL & UNITS & METHOD \\
\hline 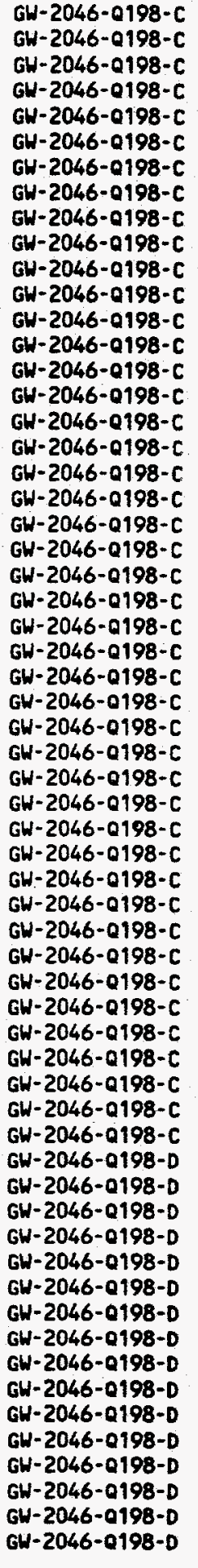 & $\begin{array}{l}01 / 23 / 98 \\
01 / 23 / 98 \\
01 / 23 / 98 \\
01 / 23 / 98 \\
01 / 23 / 98 \\
01 / 23 / 98 \\
01 / 23 / 98 \\
01 / 23 / 98 \\
01 / 23 / 98 \\
01 / 23 / 98 \\
01 / 23 / 98 \\
01 / 23 / 98 \\
01 / 23 / 98 \\
01 / 23 / 98 \\
01 / 23 / 98 \\
01 / 23 / 98 \\
01 / 23 / 98 \\
01 / 23 / 98 \\
01 / 23 / 98 \\
01 / 23 / 98 \\
011 / 23 / 98 \\
01 / 23 / 98 \\
01 / 23 / 98 \\
01 / 23 / 98 \\
01 / 23 / 98 \\
011 / 23 / 98 \\
01 / 23 / 98 \\
01 / 23 / 98 \\
01 / 23 / 98 \\
01 / 23 / 98 \\
01 / 23 / 98 \\
011 / 23 / 98 \\
01 / 23 / 98 \\
01 / 23 / 98 \\
01 / 23 / 98 \\
01 / 23 / 98 \\
011 / 23 / 98 \\
01 / 23 / 98 \\
01 / 23 / 98 \\
01 / 23 / 98 \\
01 / 23 / 98 \\
01 / 23 / 98 \\
01 / 23 / 98 \\
01 / 23 / 98 \\
01 / 23 / 98 \\
01 / 26 / 98 \\
01 / 26 / 98 \\
01 / 26 / 98 \\
01 / 26 / 98 \\
01 / 26 / 98 \\
01 / 26 / 98 \\
01 / 26 / 98 \\
01 / 26 / 98 \\
01 / 26 / 98 \\
01 / 26 / 98 \\
01 / 26 / 98 \\
01 / 26 / 98 \\
01 / 26 / 98 \\
01 / 26 / 98 \\
01 / 26 / 98 \\
01 / 26 / 98\end{array}$ & 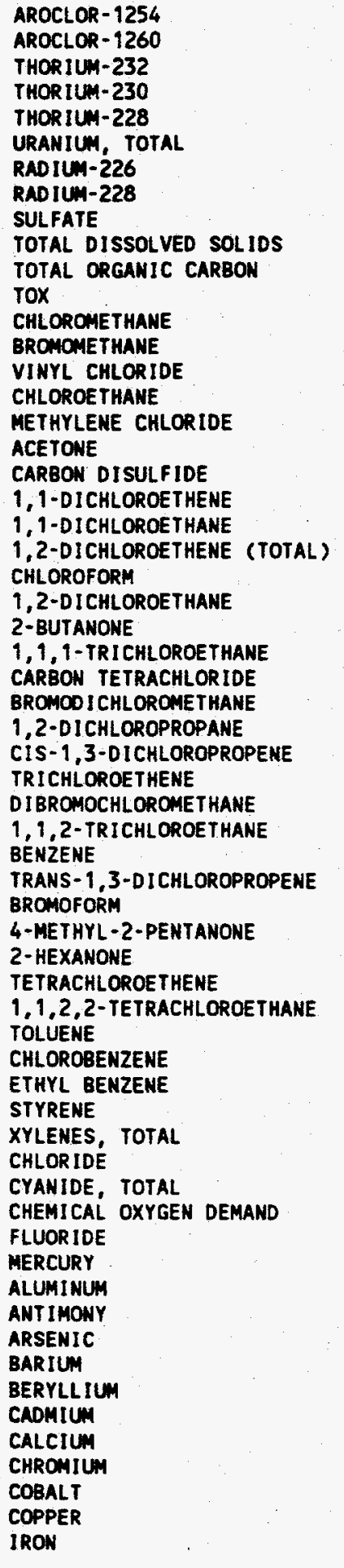 & 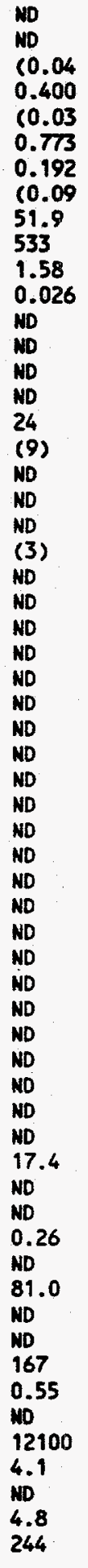 & 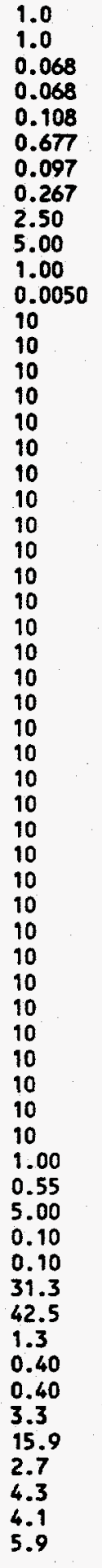 & $\begin{array}{l}U G / L \\
U G / L \\
P C I / L \\
P C I / L \\
P C I / L \\
P C I / L \\
P C I / L \\
P C I / L \\
M G / L \\
U G / L \\
U G / L \\
M G / L \\
U G / L \\
U G / L \\
U G / L \\
U G / L \\
U G / L \\
U G / L \\
U G / L \\
U G / L \\
U G / L \\
U G / L \\
U G / L \\
U G / L \\
U G / L \\
U G / L \\
U G / L \\
U G / L \\
U G / L \\
U G / L \\
U G / L \\
U G / L \\
U G / L \\
U G / L \\
U G / L \\
U G / L \\
U G / L \\
U G / L \\
U G / L \\
U G / L \\
U G / L \\
U G / L \\
U G / L \\
U G / L \\
U G / L \\
M G / L \\
U G / L \\
M G / L \\
M G / L \\
U G / L \\
U G / L \\
U G / L \\
U G / L \\
U G / L \\
U G / L \\
U G / L \\
U G / L \\
U G / L \\
U G / L \\
U G / L \\
U G / L \\
G\end{array}$ & $\begin{array}{l}\text { EPA } 8080 A \\
\text { EPA } 8080 A \\
\text { NAS-NS-3004 } \\
\text { NAS-NS-3004 } \\
\text { NAS-NS-3004 } \\
\text { ASTM } 5174-91 \\
\text { EPA-904.0 } \\
\text { EPA } 904.0 \\
\text { EPA } 300.0 \\
\text { EPA } 160.1 \\
\text { EPA } 415.1 \\
\text { EPA } 9020 \\
\text { EPA CLP } \\
\text { EPA CLP } \\
\text { EPA CLP } \\
\text { EPA CLP } \\
\text { EPA CLP } \\
\text { EPA CLP } \\
\text { EPA CLP } \\
\text { EPA CLP } \\
\text { EPA CLP } \\
\text { EPA CLP } \\
\text { EPA CLP } \\
\text { EPA CLP } \\
\text { EPA CLP } \\
\text { EPA CLP } \\
\text { EPA CLP } \\
\text { EPA CLP } \\
\text { EPA CLP } \\
\text { EPA CLP } \\
\text { EPA CLP } \\
\text { EPA CLP } \\
\text { EPA CLP } \\
\text { EPA CLP } \\
\text { EPA CLP } \\
\text { EPA CLP } \\
\text { EPA CLP } \\
\text { EPA CLP } \\
\text { EPA CLP } \\
\text { EPA CLP } \\
\text { EPA CLP } \\
\text { EPA CLP } \\
\text { EPA CLP } \\
\text { EPA CLP } \\
\text { EPA CLP } \\
\text { EPA } 300.0 \\
\text { EPA CLP } \\
\text { EPA } 410.4 \\
\text { EPA } 300.0 \\
\text { EPA CLP } \\
\text { EPA CLP } \\
\text { EPA CLP } \\
\text { EPA CLP } \\
\text { EPA CLP } \\
\text { EPA CLP } \\
\text { EPA CLP } \\
\text { EPA CLP } \\
\text { EPA CLP } \\
\text { EPA CLP } \\
\text { EPA CLP } \\
\text { EPA CLP } \\
\end{array}$ \\
\hline
\end{tabular}




\begin{tabular}{|c|c|c|c|c|c|c|}
\hline WSSRAP_ID & DATE_SAM & PARAMETER & CONC & $\mathrm{DL}$ & UNITS & METHOD \\
\hline $\begin{array}{l}G W-2046-0198-D \\
G W-2046-0198-D \\
G W-2046-0198-D \\
G W-2046-0198-D \\
G W-2046-0198-D \\
G W-2046-0198-D \\
G W-2046-0198-D \\
G W-2046-0198-D \\
G W-2046-0198-D \\
G W-2046-0198-D \\
G W-2046-0198-D \\
G W-2046-0198-D \\
G W-2046-0198-D \\
G W-2046-0198-D \\
G W-2046-0198-D \\
G W-2046-0198-D \\
G W-2046-0198-D \\
G W-2046-0198-D \\
G W-2046-0198-D \\
G W-2046-0198-D \\
G W-2046-0198-D \\
G W-2046-0198-D \\
G W-2046-0198-D \\
G W-2046-0198-D \\
G W-2046-0198-D \\
G W-2046-0198-D \\
G W-2046-0198-D \\
G W-2046-0198-D \\
G W-2046-0198-D \\
G W-2046-0198-D \\
G W-2046-0198-D \\
G W-2046-0198-D \\
G W-2046-0198-D \\
G W-2046-0198-D \\
G W-2046-0198-D \\
G W-2046-0198-D \\
G W-2046-0198-D \\
G W-2046-0198-D \\
G W-2046-0198-D \\
G H-2046-0198-D \\
G W-2046-0198-D \\
G W-2046-0198-D \\
G W-2046-0198-D \\
G W-2046-0198-D \\
G W-2046-0198-D \\
G W-2046-0198-D \\
G W-2046-0198-D \\
G W-2046-0198-D \\
G W-2046-0198-D \\
G W-2046-0198-D \\
G W-2046-0198-D \\
G W-2046-0198-D \\
G W-2046-0198-D \\
G W-2046-0198-D \\
G W-2046-0198-D \\
G W-2046-0198-D \\
G W-2046-0198-D \\
G W-2046-0198-D \\
G W-2046-0198-D \\
G W-2046-0198-D \\
G W-2046-0198-D\end{array}$ & $\begin{array}{l}01 / 26 / 98 \\
01 / 26 / 98 \\
01 / 26 / 98 \\
01 / 26 / 98 \\
01 / 26 / 98 \\
01 / 26 / 98 \\
01 / 26 / 98 \\
01 / 26 / 98 \\
01 / 26 / 98 \\
01 / 26 / 98 \\
01 / 26 / 98 \\
01 / 26 / 98 \\
01 / 26 / 98 \\
01 / 26 / 98 \\
01 / 26 / 98 \\
01 / 26 / 98 \\
01 / 26 / 98 \\
01 / 26 / 98 \\
01 / 26 / 98 \\
01 / 26 / 98 \\
01 / 26 / 98 \\
01 / 26 / 98 \\
01 / 26 / 98 \\
01 / 26 / 98 \\
01 / 26 / 98 \\
01 / 26 / 98 \\
01 / 26 / 98 \\
01 / 26 / 98 \\
01 / 26 / 98 \\
011 / 26 / 98 \\
01 / 26 / 98 \\
01 / 26 / 98 \\
01 / 26 / 98 \\
01 / 26 / 98 \\
01 / 26 / 98 \\
01 / 26 / 98 \\
01 / 26 / 98 \\
01 / 26 / 98 \\
01 / 26 / 98 \\
01 / 26 / 98 \\
01 / 26 / 98 \\
01 / 26 / 98 \\
01 / 26 / 98 \\
01 / 26 / 98 \\
01 / 26 / 98 \\
01 / 26 / 98 \\
01 / 26 / 98 \\
01 / 26 / 98 \\
01 / 26 / 98 \\
01 / 26 / 98 \\
01 / 26 / 98 \\
01 / 26 / 98 \\
01 / 26 / 98 \\
01 / 26 / 98 \\
01 / 26 / 98 \\
01 / 26 / 98 \\
01 / 26 / 98 \\
01 / 26 / 98 \\
01 / 26 / 98 \\
01 / 26 / 98 \\
01 / 26 / 98\end{array}$ & 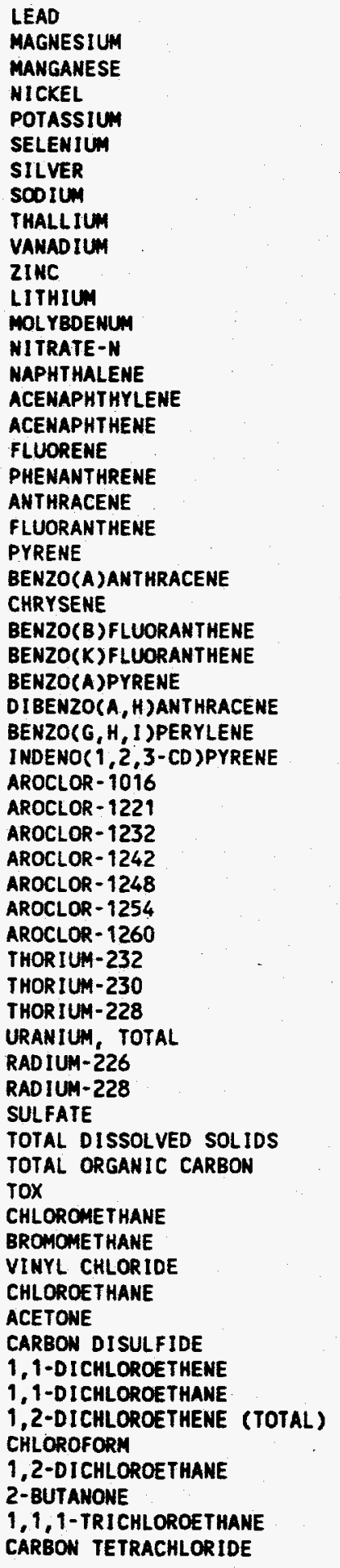 & $\begin{array}{l}\text { ND } \\
38800 \\
27.1 \\
\text { ND } \\
\text { ND } \\
\text { ND } \\
\text { ND } \\
25900 \\
\text { ND } \\
14.8 \\
9.8 \\
14.4 \\
\text { ND } \\
1.91 \\
\text { ND } \\
\text { ND } \\
\text { ND } \\
\text { ND } \\
\text { ND } \\
\text { ND } \\
\text { ND } \\
\text { ND } \\
\text { ND } \\
\text { ND } \\
\text { ND } \\
\text { ND } \\
\text { ND } \\
\text { ND } \\
\text { ND } \\
\text { ND } \\
\text { ND } \\
\text { ND } \\
\text { ND } \\
\text { ND } \\
\text { ND } \\
\text { ND } \\
\text { ND } \\
\text { CO.05 } \\
0.435 \\
\text { CO.08 } \\
0.854 \\
0.287 \\
\text { CO.15 } \\
50.8 \\
532 \\
1.34 \\
1.36 \\
\text { ND } \\
\text { ND } \\
\text { ND } \\
\text { ND } \\
\text { C8) } \\
\text { ND } \\
\text { ND } \\
\text { ND } \\
\text { C3) } \\
\text { ND } \\
\text { ND } \\
\text { ND } \\
\text { ND } \\
\text { ND } \\
\text { ND }\end{array}$ & $\begin{array}{l}0.90 \\
54.5 \\
1.0 \\
14.6 \\
2180 \\
2.2 \\
6.0 \\
34.4 \\
1.8 \\
2.9 \\
2.3 \\
4.1 \\
11.1 \\
0.25 \\
5.0 \\
5.0 \\
5.0 \\
5.0 \\
5.0 \\
5.0 \\
5.0 \\
5.0 \\
5.0 \\
5.0 \\
5.0 \\
5.0 \\
5.0 \\
5.0 \\
5\end{array}$ & 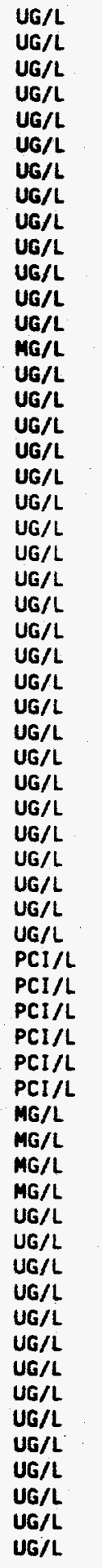 & $\begin{array}{l}\text { EPA CLP } \\
\text { EPA CLP } \\
\text { EPA CLP } \\
\text { EPA CLP } \\
\text { EPA CLP } \\
\text { EPA CLP } \\
\text { EPA CLP } \\
\text { EPA CLP } \\
\text { EPA CLP } \\
\text { EPA CLP } \\
\text { EPA CLP } \\
\text { EPA CLP } \\
\text { EPA CLP } \\
\text { EPA } 353.1 \\
\text { EPA } 8310 \\
\text { EPA } 8310 \\
\text { EPA } 8310 \\
\text { EPA } 8310 \\
\text { EPA } 8310 \\
\text { EPA } 8310 \\
\text { EPA } 8310 \\
\text { EPA } 8310 \\
\text { EPA } 8310 \\
\text { EPA } 8310 \\
\text { EPA } 8310 \\
\text { EPA } 8310 \\
\text { EPA } 8310 \\
\text { EPA } 8310 \\
\text { EPA } 8310 \\
\text { EPA } 8310 \\
\text { EPA } 8080 A \\
\text { EPA } 8080 A \\
\text { EPA } 8080 A \\
\text { EPA } 8080 A \\
\text { EPA } 8080 A \\
\text { EPA } 8080 A \\
\text { EPA } 8080 A \\
\text { NAS-NS-3004 } \\
\text { NAS-NS-3004 } \\
\text { NAS-NS-3004 } \\
\text { ASTM } 5174-91 \\
\text { EPA } 904.0 \\
\text { EPA } 904.0 \\
\text { EPA } 300.0 \\
\text { EPA } 160.1 \\
\text { EPA } 415.1 \\
\text { EPA } 9020 \\
\text { EPA CLP } \\
\text { EPA CLP } \\
\text { EPA CLP } \\
\text { EPA CLP } \\
\text { EPA CLP } \\
\text { EPA CLP } \\
\text { EPA CLP } \\
\text { EPA CLP } \\
\text { EPA CLP } \\
\text { EPA CLP } \\
\text { EPA CLP } \\
\text { EPA CLP } \\
\text { EPA CLP } \\
\text { EPA CLP }\end{array}$ \\
\hline
\end{tabular}




\begin{tabular}{|c|c|c|c|c|c|c|}
\hline WSSRAP_ID & DATE_SAM & PARAMETER & CONC & DL & UNITS & METHOD \\
\hline $\begin{array}{l}G W-2046-0198-D \\
G W-2046-0198-D \\
G W-2046-0198-D \\
G W-2046-0198-D \\
G W-2046-0198-D \\
G H-2046-0198-D \\
G W-2046-0198-D \\
G W-2046-0198-D \\
G W-2046-0198-D \\
G W-2046-0198-D \\
G W-2046-0198-D \\
G W-2046-0198-D \\
G W-2046-0198-D \\
G W-2046-0198-D \\
G H-2046-0198-D \\
G W-2046-0198-D \\
G W-2046-0198-D \\
G W-2046-0198-D \\
G W-2046-0198-D \\
G W-2047-0198-A \\
G W-2047-0198-A \\
G W-2047-0198-A \\
G W-2047-0198-A \\
G W-2047-0198-A \\
G W-2047-0198-A \\
G W-2047-0198-A \\
G W-2047-0198-A \\
G W-2047-0198-A \\
G W-2047-0198-A \\
G W-2047-0198-A \\
G W-2047-0198-A \\
G W-2047-0198-A \\
G W-2047-0198-A \\
G W-2047-0198-A \\
G W-2047-0198-A \\
G W-2047-0198-A \\
G W-2047-0198-A \\
G W-2047-0198-A \\
G W-2047-0198-A \\
G W-2047-0198-A \\
G W-2047-0198-A \\
G W-2047-0198-A \\
G W-2047-0198-A \\
G W-2047-0198-A \\
G W-2047-0198-A \\
G W-2047-0198-A \\
G W-2047-0198-A \\
G W-2047-0198-A \\
G W-2047-0198-A \\
G W-2047-0198-A \\
G W-2047-0198-A \\
G W-2047-0198-A \\
G W-2047-0198-A \\
G W-2047-0198-A \\
G W-2047-0198-A \\
G W-2047-0198-A \\
G W-2047-0198-A \\
G W-2047-0198-A \\
G W-2047-0198-A \\
G W-2047-0198-A \\
G W-2047-0198-A\end{array}$ & $\begin{array}{l}01 / 26 / 98 \\
01 / 26 / 98 \\
01 / 26 / 98 \\
01 / 26 / 98 \\
01 / 26 / 98 \\
01 / 26 / 98 \\
01 / 26 / 98 \\
01 / 26 / 98 \\
01 / 26 / 98 \\
01 / 26 / 98 \\
01 / 26 / 98 \\
01 / 26 / 98 \\
01 / 26 / 98 \\
01 / 26 / 98 \\
01 / 26 / 98 \\
01 / 26 / 98 \\
01 / 26 / 98 \\
01 / 26 / 98 \\
01 / 26 / 98 \\
011 / 21 / 98 \\
01 / 21 / 98 \\
01 / 21 / 98 \\
01 / 21 / 98 \\
01 / 21 / 98 \\
011 / 21 / 98 \\
01 / 21 / 98 \\
01 / 21 / 98 \\
01 / 21 / 98 \\
01 / 21 / 98 \\
01 / 21 / 98 \\
01 / 21 / 98 \\
01 / 21 / 98 \\
01 / 21 / 98 \\
01 / 21 / 98 \\
01 / 21 / 98 \\
01 / 21 / 98 \\
01 / 21 / 98 \\
01 / 21 / 98 \\
01 / 211 / 98 \\
01 / 21 / 98 \\
01 / 21 / 98 \\
01 / 21 / 98 \\
01 / 211998 \\
01 / 21 / 98 \\
01 / 21 / 98 \\
01 / 21 / 98 \\
01 / 211 / 98 \\
01 / 211 / 98 \\
01 / 21 / 98 \\
01 / 21 / 98 \\
01 / 21 / 98 \\
01 / 211988 \\
01 / 21198 \\
01 / 21 / 98 \\
01 / 21 / 98 \\
01 / 21 / 98 \\
01 / 21 / 98 \\
01 / 211998 \\
01 / 21 / 98 \\
01 / 21 / 98 \\
01 / 21 / 98\end{array}$ & 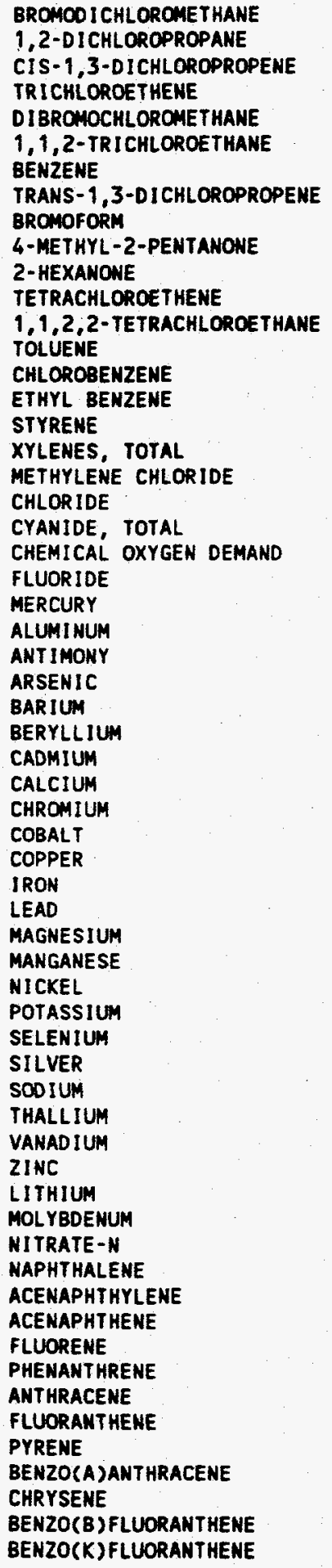 & 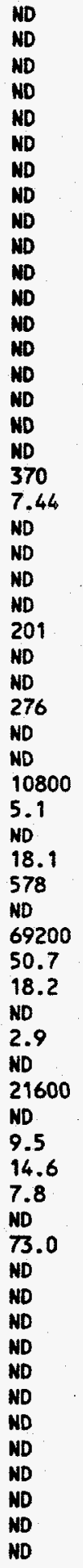 & $\begin{array}{l}10 \\
10 \\
10 \\
10 \\
10 \\
10 \\
10 \\
10 \\
10 \\
10 \\
10 \\
10 \\
10 \\
10 \\
10 \\
10 \\
10 \\
10 \\
25 \\
0.40 \\
0.55 \\
5.00 \\
0.20 \\
0.10 \\
31.3 \\
42.5 \\
1.3 \\
0.40 \\
0.40 \\
3.3 \\
15.9 \\
2.7 \\
4.3 \\
4.1 \\
5.9 \\
0.90 \\
54.5 \\
1.0 \\
14.6 \\
2180 \\
2.2 \\
6.0 \\
34.4 \\
5.0 \\
5.0 \\
5.0 \\
2.9 \\
2.3 \\
4.1 \\
11.1 \\
12.5 \\
5.0 \\
5.0 \\
5.0 \\
5.0 \\
5.0 \\
5.0 \\
5\end{array}$ & $\begin{array}{l}U G / L \\
U G / L \\
U G / L \\
U G / L \\
U G / L \\
U G / L \\
U G / L \\
U G / L \\
U G / L \\
U G / L \\
U G / L \\
U G / L \\
U G / L \\
U G / L \\
U G / L \\
U G / L \\
U G / L \\
U G / L \\
U G / L \\
M G / L \\
U G / L \\
U G / L \\
M G / L \\
U G / L \\
U G / L \\
U G / L \\
U G / L \\
U G / L \\
U G / L \\
U G / L \\
U G / L \\
U G / L \\
U G / L \\
U G / L \\
U G / L \\
U G / L \\
U G / L \\
U G / L \\
U G / L \\
U G / L \\
U G / L \\
U G / L \\
U G / L \\
U G / L \\
U G / L \\
U G / L \\
U G / L \\
U G / L \\
U G / L \\
U G / L \\
U G / L \\
U G / L \\
U G / L \\
U G / L \\
U G / L \\
U G / L \\
U G / L \\
U G / L \\
U G / L \\
U\end{array}$ & $\begin{array}{l}\text { EPA CLP } \\
\text { EPA CLP } \\
\text { EPA CLP } \\
\text { EPA CLP } \\
\text { EPA CLP } \\
\text { EPA CLP } \\
\text { EPA CLP } \\
\text { EPA CLP } \\
\text { EPA CLP } \\
\text { EPA CLP } \\
\text { EPA CLP } \\
\text { EPA CLP } \\
\text { EPA CLP } \\
\text { EPA CLP } \\
\text { EPA CLP } \\
\text { EPA CLP } \\
\text { EPA CLP } \\
\text { EPA CLP } \\
\text { EPA CLP } \\
\text { EPA } 300.0 \\
\text { EPA CLP } \\
\text { EPA } 410.4 \\
\text { EPA } 300.0 \\
\text { EPA CLP } \\
\text { EPA CLP } \\
\text { EPA CLP } \\
\text { EPA CLP } \\
\text { EPA CLP } \\
\text { EPA CLP } \\
\text { EPA CLP } \\
\text { EPA CLP } \\
\text { EPA CLP } \\
\text { EPA CLP } \\
\text { EPA CLP } \\
\text { EPA CLP } \\
\text { EPA CLP } \\
\text { EPA CLP } \\
\text { EPA CLP } \\
\text { EPA CLP } \\
\text { EPA CLP } \\
\text { EPA CLP } \\
\text { EPA CLP } \\
\text { EPA CLP } \\
\text { EPA CLP } \\
\text { EPA CLP } \\
\text { EPA CLP } \\
\text { EPA CLP } \\
\text { EPA CLP } \\
\text { EPA } 353.1 \\
\text { EPA } 8310 \\
\text { EPA } 8310 \\
\text { EPA } 8310 \\
\text { EPA } 8310 \\
\text { EPA } 8310 \\
\text { EPA } 8310 \\
\text { EPA } 8310 \\
\text { EPA } 8310 \\
\text { EPA } 8310 \\
\text { EPA } 8310 \\
\text { EPA } 8310 \\
\text { EPA } 8310\end{array}$ \\
\hline
\end{tabular}




\begin{tabular}{|c|c|c|c|c|c|c|}
\hline USSRAP_10 & DATE_SAM & PARAMETER & CONC & $\mathrm{DL}$ & UNITS & METHOO \\
\hline 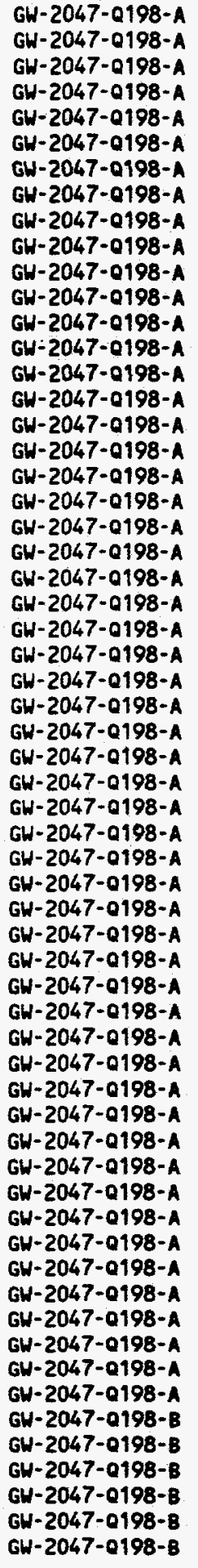 & $\begin{array}{l}01 / 21 / 98 \\
01 / 21 / 98 \\
01 / 21 / 98 \\
01 / 21 / 98 \\
01 / 21 / 98 \\
01 / 21 / 98 \\
01 / 21 / 98 \\
01 / 21 / 98 \\
01 / 21 / 98 \\
01 / 21 / 98 \\
01 / 21 / 98 \\
01 / 21 / 98 \\
01 / 21 / 98 \\
01 / 21 / 98 \\
01 / 21 / 98 \\
01 / 21 / 98 \\
01 / 21 / 98 \\
01 / 21 / 98 \\
01 / 21 / 98 \\
01 / 21 / 98 \\
01 / 21 / 98 \\
01 / 21 / 98 \\
01 / 21 / 98 \\
01 / 21 / 98 \\
01 / 21 / 98 \\
01 / 21 / 98 \\
01 / 21 / 98 \\
01 / 21 / 98 \\
01 / 21 / 98 \\
01 / 21 / 98 \\
01 / 21 / 98 \\
01 / 21 / 98 \\
01 / 21 / 98 \\
01 / 21 / 98 \\
01 / 21 / 98 \\
01 / 21 / 98 \\
01 / 21 / 98 \\
01 / 21 / 98 \\
01 / 21 / 98 \\
01 / 21 / 98 \\
01 / 21 / 98 \\
01 / 21 / 98 \\
01 / 21 / 98 \\
01 / 21 / 98 \\
01 / 21 / 98 \\
01 / 21 / 98 \\
01 / 21 / 98 \\
01 / 21 / 98 \\
01 / 21 / 98 \\
01 / 21 / 98 \\
01 / 21 / 98 \\
01 / 21 / 98 \\
01 / 21 / 98 \\
01 / 21 / 98 \\
01 / 21 / 98 \\
01 / 22 / 98 \\
01 / 22 / 98 \\
01 / 22 / 98 \\
01 / 22 / 98 \\
01 / 22 / 98 \\
01 / 22 / 98\end{array}$ & 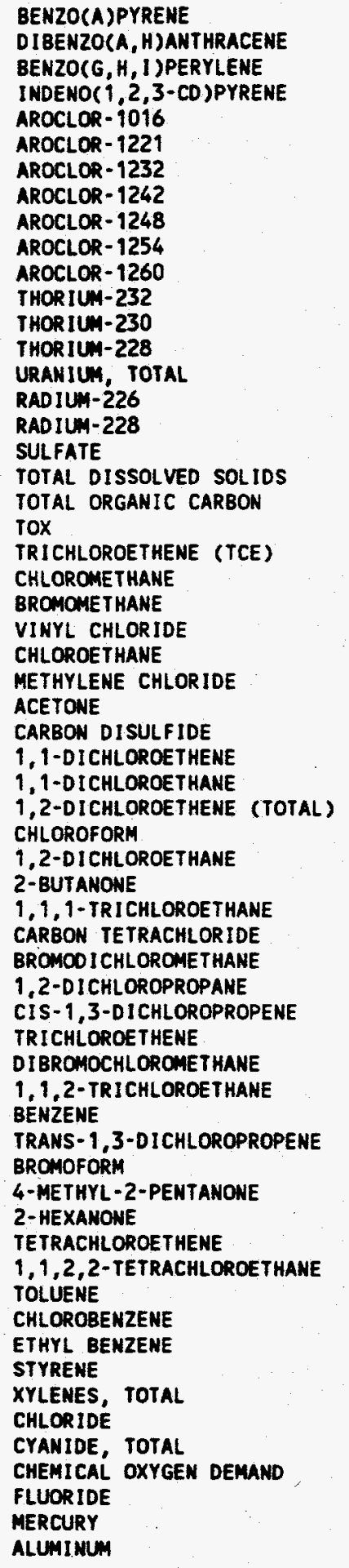 & 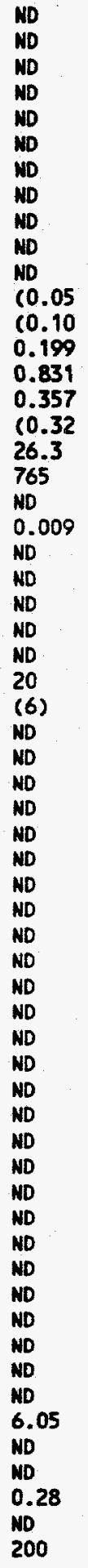 & 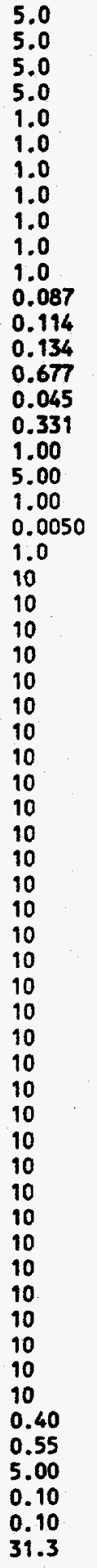 & 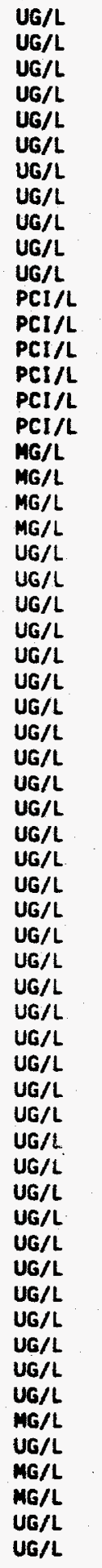 & $\begin{array}{l}\text { EPA } 8310 \\
\text { EPA } 8310 \\
\text { EPA } 8310 \\
\text { EPA } 8310 \\
\text { EPA } 8080 A \\
\text { EPA } 8080 A \\
\text { EPA } 8080 A \\
\text { EPA } 8080 A \\
\text { EPA } 8080 A \\
\text { EPA } 8080 A \\
\text { EPA } 8080 A \\
\text { NAS-NS-3004 } \\
\text { NAS-NS-3004 } \\
\text { NAS-NS-3004 } \\
\text { ASTM } 5174-91 \\
\text { EPA } 904.0 \\
\text { EPA } 904.0 \\
\text { EPA } 300.0 \\
\text { EPA } 160.1 \\
\text { EPA } 415.1 \\
\text { EPA } 9020 \\
\text { EPA } 8260 \\
\text { EPA CLP } \\
\text { EPA CLP } \\
\text { EPA CLP } \\
\text { EPA CLP } \\
\text { EPA CLP } \\
\text { EPA CLP } \\
\text { EPA CLP } \\
\text { EPA CLP } \\
\text { EPA CLP } \\
\text { EPA CLP } \\
\text { EPA CLP } \\
\text { EPA CLP } \\
\text { EPA CLP } \\
\text { EPA CLP } \\
\text { EPA CLP } \\
\text { EPA CLP } \\
\text { EPA CLP } \\
\text { EPA CLP } \\
\text { EPA CLP } \\
\text { EPA CLP } \\
\text { EPA CLP } \\
\text { EPA CLP } \\
\text { EPA CLP } \\
\text { EPA CLP } \\
\text { EPA CLP } \\
\text { EPA CLP } \\
\text { EPA CLP } \\
\text { EPA CLP } \\
\text { EPA CLP } \\
\text { EPA CLP } \\
\text { EPA CLP } \\
\text { EPA CLP } \\
\text { EPA CLP } \\
\text { EPA } 300.0 \\
\text { EPA CLP } \\
\text { EPA } 410.4 \\
\text { EPA } 300.0 \\
\text { EPA CLP } \\
\text { EPA CLP } \\
\text { A }\end{array}$ \\
\hline
\end{tabular}




\begin{tabular}{|c|c|c|c|c|c|c|}
\hline HSSRAP_ID & DATE_SAM & PARAMETER & CONC & $\mathrm{DL}$ & UNITS & METHOO \\
\hline $\begin{array}{l}\text { GW-2047-0198-B } \\
G W-2047-0198-B \\
G W-2047-0198-B \\
G W-2047-0198-B \\
G W-2047-0198-B \\
G W-2047-0198-B \\
G W-2047-0198-B \\
G W-2047-0198-B \\
G W-2047-0198-B \\
G W-2047-0198-B \\
G W-2047-0198-B \\
G W-2047-0198-B \\
G W-2047-0198-B \\
G W-2047-0198-B \\
G W-2047-0198-B \\
G W-2047-0198-B \\
G W-2047-0198-B \\
G W-2047-0198-B \\
G W-2047-0198-B \\
G W-2047-0198-B \\
G W-2047-0198-B \\
G W-2047-0198-B \\
G W-2047-0198-B \\
G W-2047-0198-B \\
G W-2047-0198-B \\
G W-2047-0198-B \\
G W-2047-0198-B \\
G W-2047-0198-B \\
G W-2047-0198-B \\
G W-2047-0198-B \\
G W-2047-0198-B \\
G W-2047-0198-B \\
G W-2047-0198-B \\
G W-2047-0198-B \\
G W-2047-0198-B \\
G W-2047-0198-B \\
G W-2047-0198-B \\
G W-2047-0198-B \\
G W-2047-0198-B \\
G W-2047-0198-B \\
G W-2047-0198-8 \\
G W-2047-0198-B \\
G W-2047-0198-B \\
G W-2047-0198-B \\
G W-2047-0198-B \\
G W-2047-0198-B \\
G W-2047-0198-B \\
G W-2047-0198-B \\
G W-2047-0198-B \\
G W-2047-0198-B \\
G W-2047-0198-B \\
G W-2047-0198-B \\
G W-2047-0198-B \\
G W-2047-0198-B \\
G W-2047-0198-B \\
G W-2047-0198-B \\
G W-2047-0198-B \\
G W-2047-0198-B \\
G W-2047-0198-B \\
G W-2047-0198-B \\
G W-2047-0198-B\end{array}$ & $\begin{array}{l}01 / 22 / 98 \\
01 / 22 / 98 \\
01 / 22 / 98 \\
01 / 22 / 98 \\
01 / 22 / 98 \\
01 / 22 / 98 \\
01 / 22 / 98 \\
01 / 22 / 98 \\
01 / 22 / 98 \\
01 / 22 / 98 \\
01 / 22 / 98 \\
01 / 22 / 98 \\
01 / 22 / 98 \\
01 / 22 / 98 \\
01 / 22 / 98 \\
01 / 22 / 98 \\
01 / 22 / 98 \\
01 / 22 / 98 \\
01 / 22 / 98 \\
01 / 22 / 98 \\
01 / 22 / 98 \\
01 / 22 / 98 \\
01 / 22 / 98 \\
01 / 22 / 98 \\
01 / 22 / 98 \\
01 / 22 / 98 \\
01 / 22 / 98 \\
01 / 22 / 98 \\
01 / 22 / 98 \\
01 / 22 / 98 \\
01 / 22 / 98 \\
01 / 22 / 98 \\
01 / 22 / 98 \\
01 / 22 / 98 \\
01 / 22 / 98 \\
01 / 22 / 98 \\
01 / 22 / 98 \\
01 / 22 / 98 \\
01 / 22 / 98 \\
01 / 22 / 98 \\
01 / 22 / 98 \\
01 / 22 / 98 \\
01 / 22 / 98 \\
01 / 22 / 98 \\
01 / 22 / 98 \\
01 / 22 / 98 \\
01 / 22 / 98 \\
01 / 22 / 98 \\
01 / 22 / 98 \\
01 / 22 / 98 \\
01 / 22 / 98 \\
01 / 22 / 98 \\
01 / 22 / 98 \\
01 / 22 / 98 \\
01 / 22 / 98 \\
01 / 22 / 98 \\
01 / 22 / 98 \\
01 / 22 / 98 \\
01 / 22 / 98 \\
01 / 22 / 98 \\
01 / 22 / 98\end{array}$ & 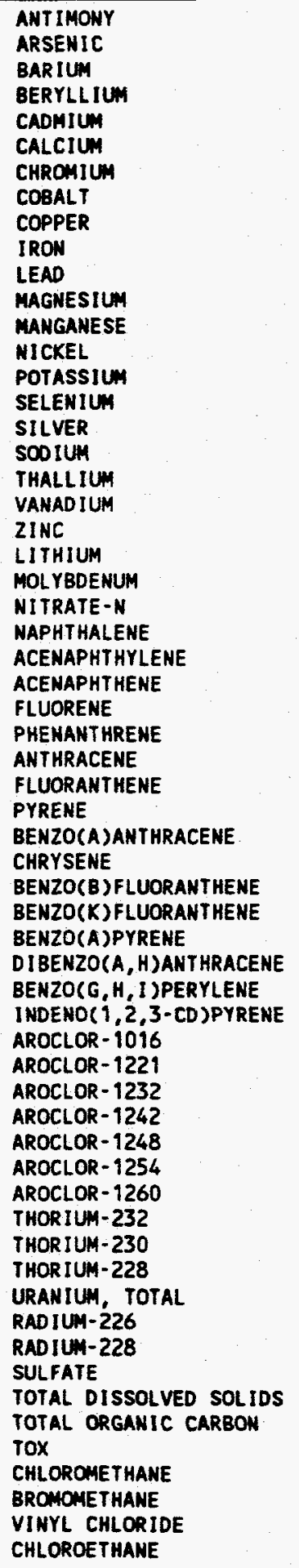 & $\begin{array}{l}\text { ND } \\
\text { ND } \\
263 \\
\text { ND } \\
\text { ND } \\
10500 \\
4.8 \\
\text { ND } \\
14.3 \\
\text { S70 } \\
\text { ND } \\
65700 \\
\text { W6.9 } \\
\text { ND } \\
2320 \\
2.4 \\
\text { ND } \\
21800 \\
\text { ND } \\
11.7 \\
14.7 \\
14.5 \\
\text { ND } \\
115 \\
\text { ND } \\
\text { ND } \\
\text { ND } \\
\text { ND } \\
\text { ND } \\
\text { ND } \\
\text { ND } \\
\text { ND } \\
\text { ND } \\
\text { ND } \\
\text { ND } \\
\text { ND } \\
\text { ND } \\
\text { ND } \\
\text { ND } \\
\text { ND } \\
\text { ND } \\
\text { ND } \\
\text { ND } \\
\text { ND } \\
\text { ND } \\
\text { ND } \\
\text { ND } \\
0.043 \\
\text { No.08 } \\
\text { O.05 } \\
0.948 \\
0.468 \\
0.436 \\
23.2 \\
843 \\
\text { ND } \\
0.030 \\
\text { ND } \\
\text { ND } \\
\text { ND } \\
\text { ND }\end{array}$ & 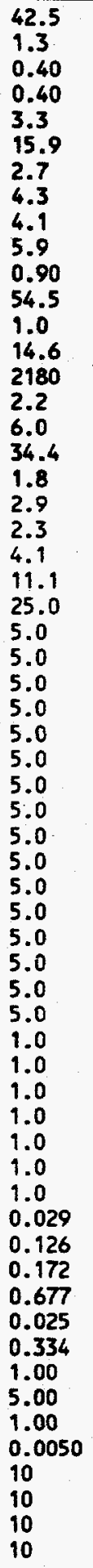 & 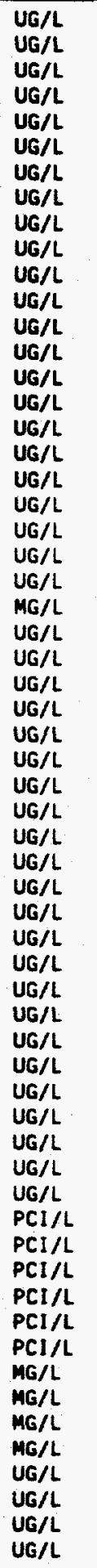 & $\begin{array}{l}\text { EPA CLP } \\
\text { EPA CLP } \\
\text { EPA CLP } \\
\text { EPA CLP } \\
\text { EPA CLP } \\
\text { EPA CLP } \\
\text { EPA CLP } \\
\text { EPA CLP } \\
\text { EPA CLP } \\
\text { EPA CLP } \\
\text { EPA CLP } \\
\text { EPA CLP } \\
\text { EPA CLP } \\
\text { EPA CLP } \\
\text { EPA CLP } \\
\text { EPA CLP } \\
\text { EPA CLP } \\
\text { EPA CLP } \\
\text { EPA CLP } \\
\text { EPA CLP } \\
\text { EPA CLP } \\
\text { EPA CLP } \\
\text { EPA CLP } \\
\text { EPA } 353.1 \\
\text { EPA } 8310 \\
\text { EPA } 8310 \\
\text { EPA } 8310 \\
\text { EPA } 8310 \\
\text { EPA } 8310 \\
\text { EPA } 8310 \\
\text { EPA } 8310 \\
\text { EPA } 8310 \\
\text { EPA } 8310 \\
\text { EPA } 8310 \\
\text { EPA } 8310 \\
\text { EPA } 8310 \\
\text { EPA } 8310 \\
\text { EPA } 8310 \\
\text { EPA } 8310 \\
\text { EPA } 8310 \\
\text { EPA } 8080 A \\
\text { EPA } 8080 A \\
\text { EPA } 8080 A \\
\text { EPA } 8080 A \\
\text { EPA } 8080 A \\
\text { EPA } 8080 A \\
\text { EPA } 8080 A \\
\text { NAS- NS-3004 } \\
\text { NAS-NS-3004 } \\
\text { HAS-NS-3004 } \\
\text { ASTM } 5174-91 \\
\text { EPA } 904.0 \\
\text { EPA } 904.0 \\
\text { EPA } 300.0 \\
\text { EPA } 160.1 \\
\text { EPA } 415.1 \\
\text { EPA } 9020 \\
\text { EPA CLP } \\
\text { EPA CLP } \\
\text { EPA CLP } \\
\text { EPA CLP } \\
\text { EPA }\end{array}$ \\
\hline
\end{tabular}




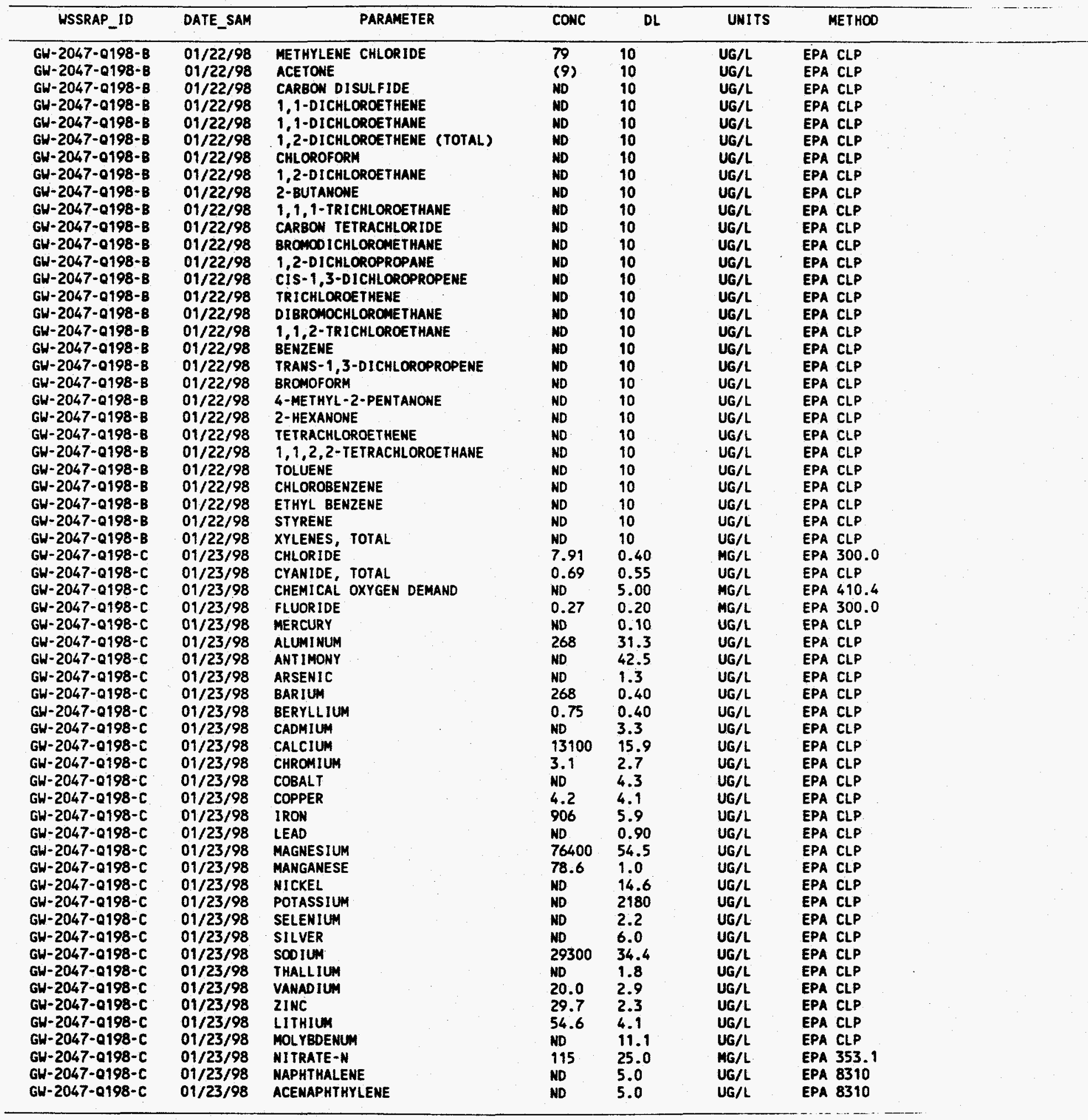




\begin{tabular}{|c|c|c|c|c|c|c|}
\hline WSSRAP_ID & DATE_SAM & PARAMETER & CONC & $D L$ & UNITS & METHOO \\
\hline $\begin{array}{l}G W-2047-0198-C \\
G W-2047-0198-C \\
G W-2047-0198-C \\
G W-2047-0198-C \\
G H-2047-0198-C \\
G W-2047-0198-C \\
G H-2047-0198-C \\
G W-2047-0198-C \\
G W-2047-0198-C \\
G W-2047-0198-C \\
G W-2047-0198-C \\
G W-2047-0198-C \\
G W-2047-0198-C \\
G W-2047-0198-C \\
G W-2047-0198-C \\
G W-2047-0198-C \\
G W-2047-0198-C \\
G W-2047-0198-C \\
G W-2047-0198-C \\
G W-2047-0198-C \\
G W-2047-0198-C \\
G W-2047-0198-C \\
G W-2047-0198-C \\
G W-2047-0198-C \\
G H-2047-0198-C \\
G W-2047-0198-C \\
G W-2047-0198-C \\
G W-2047-0198-C \\
G W-2047-0198-C \\
G W-2047-0198-C \\
G W-2047-0198-C \\
G W-2047-0198-C \\
G W-2047-0198-C \\
G W-2047-0198-C \\
G W-2047-0198-C \\
G W-2047-0198-C \\
G W-2047-0198-C \\
G W-2047-0198-C \\
G W-2047-0198-C \\
G W-2047-0198-C \\
G W-2047-0198-C \\
G W-2047-0198-C \\
G W-2047-0198-C \\
G W-2047-0198-C \\
G W-2047-0198-C \\
G W-2047-0198-C \\
G W-2047-0198-C \\
G W-2047-0198-C \\
G W-2047-0198-C \\
G W-2047-0198-C \\
G W-2047-0198-C \\
G W-2047-0198-C \\
G W-2047-0198-C \\
G W-2047-0198-C \\
G W-2047-0198-C \\
G W-2047-0198-C \\
G W-2047-0198-C \\
G W-2047-0198-C \\
G W-2047-0198-C \\
G W-2047-0198-C \\
G W-2047-0198-C\end{array}$ & $\begin{array}{l}01 / 23 / 98 \\
01 / 23 / 98 \\
01 / 23 / 98 \\
01 / 23 / 98 \\
01 / 23 / 98 \\
01 / 23 / 98 \\
01 / 23 / 98 \\
01 / 23 / 98 \\
01 / 23 / 98 \\
01 / 23 / 98 \\
01 / 23 / 98 \\
01 / 23 / 98 \\
01 / 23 / 98 \\
01 / 23 / 98 \\
01 / 23 / 98 \\
01 / 23 / 98 \\
01 / 23 / 98 \\
01 / 23 / 98 \\
01 / 23 / 98 \\
01 / 23 / 98 \\
01 / 23 / 98 \\
01 / 23 / 98 \\
01 / 23 / 98 \\
01 / 23 / 98 \\
01 / 23 / 98 \\
01 / 23 / 98 \\
01 / 23 / 98 \\
01 / 23 / 98 \\
01 / 23 / 98 \\
01 / 23 / 98 \\
01 / 23 / 98 \\
01 / 23 / 98 \\
01 / 23 / 98 \\
01 / 23 / 98 \\
01 / 23 / 98 \\
01 / 23 / 98 \\
01 / 23 / 98 \\
01 / 23 / 98 \\
01 / 23 / 98 \\
01 / 23 / 98 \\
01 / 23 / 98 \\
01 / 23 / 98 \\
01 / 23 / 98 \\
01 / 23 / 98 \\
01 / 23 / 98 \\
01 / 23 / 98 \\
01 / 23 / 98 \\
01 / 23 / 98 \\
01 / 23 / 98 \\
01 / 23 / 98 \\
01 / 23 / 98 \\
01 / 23 / 98 \\
01 / 23 / 98 \\
01 / 23 / 98 \\
01 / 23 / 98 \\
01 / 23 / 98 \\
01 / 23 / 98 \\
01 / 23 / 98 \\
01 / 23 / 98 \\
01 / 23 / 98 \\
01 / 23 / 98\end{array}$ & 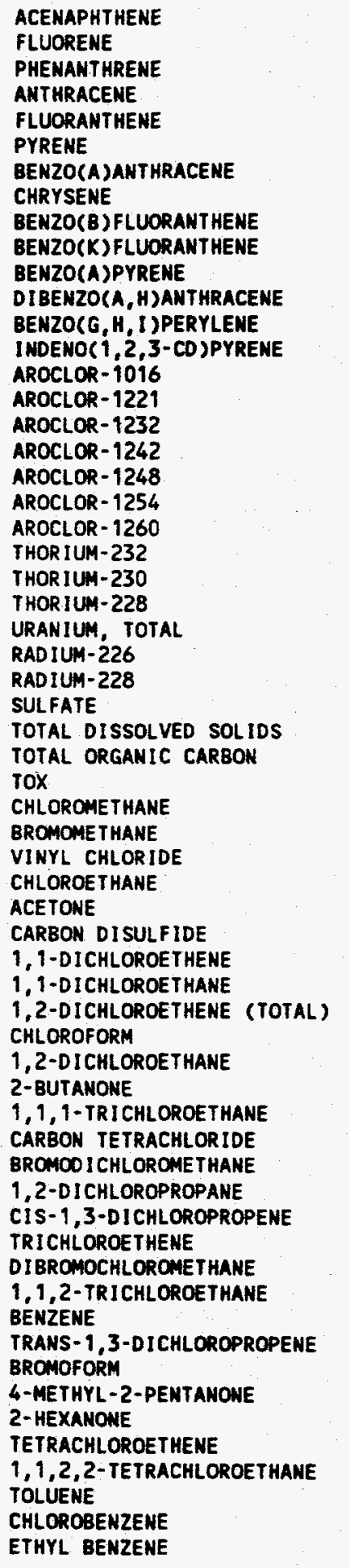 & 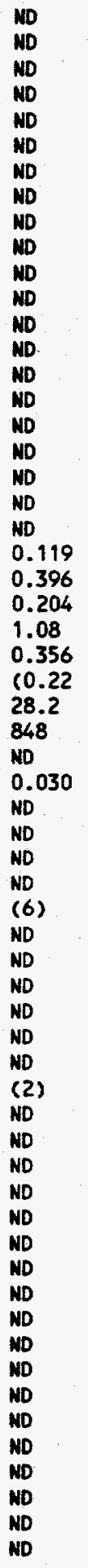 & 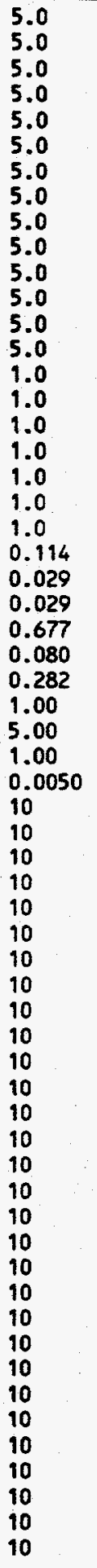 & 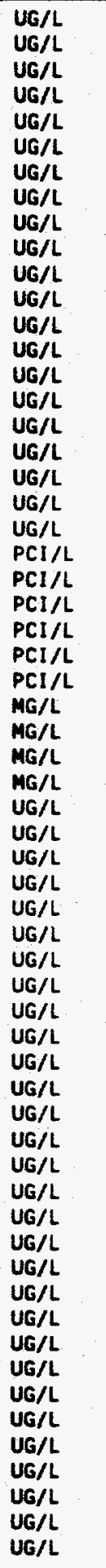 & $\begin{array}{l}\text { EPA } 8310 \\
\text { EPA } 8310 \\
\text { EPA } 8310 \\
\text { EPA } 8310 \\
\text { EPA } 8310 \\
\text { EPA } 8310 \\
\text { EPA } 8310 \\
\text { EPA } 8310 \\
\text { EPA } 8310 \\
\text { EPA } 8310 \\
\text { EPA } 8310 \\
\text { EPA } 8310 \\
\text { EPA } 8310 \\
\text { EPA } 8310 \\
\text { EPA } 8080 A \\
\text { EPA } 8080 A \\
\text { EPA } 8080 A \\
\text { EPA } 8080 A \\
\text { EPA } 8080 A \\
\text { EPA } 8080 A \\
\text { EPA } 8080 A \\
\text { NAS-NS-3004 } \\
\text { NAS-NS-3004 } \\
\text { NAS-NS-3004 } \\
\text { ASTM } 5174-91 \\
\text { EPA } 904.0 \\
\text { EPA } 904.0 \\
\text { EPA } 300.0 \\
\text { EPA } 160.1 \\
\text { EPA } 415.1 \\
\text { EPA } 9020 \\
\text { EPA CLP } \\
\text { EPA CLP } \\
\text { EPA CLP } \\
\text { EPA CLP } \\
\text { EPA CLP } \\
\text { EPA CLP } \\
\text { EPA CLP } \\
\text { EPA CLP } \\
\text { EPA CLP } \\
\text { EPA CLP } \\
\text { EPA CLP } \\
\text { EPA CLP } \\
\text { EPA CLP } \\
\text { EPA CLP } \\
\text { EPA CLP } \\
\text { EPA CLP } \\
\text { EPA CLP } \\
\text { EPA CLP } \\
\text { EPA CLP } \\
\text { EPA CLP } \\
\text { EPA CLP } \\
\text { EPA CLP } \\
\text { EPA CLP } \\
\text { EPA CLP } \\
\text { EPA CLP } \\
\text { EPA CLP } \\
\text { EPA CLP } \\
\text { EPA CLP } \\
\text { EPA CLP } \\
\text { EPA CLP } \\
\text { CPA }\end{array}$ \\
\hline
\end{tabular}




\begin{tabular}{|c|c|c|c|c|c|c|}
\hline WSSRAP_ID & DATE_SAM & PARAMETER & CONC & $D L$ & UNITS & METHOD \\
\hline 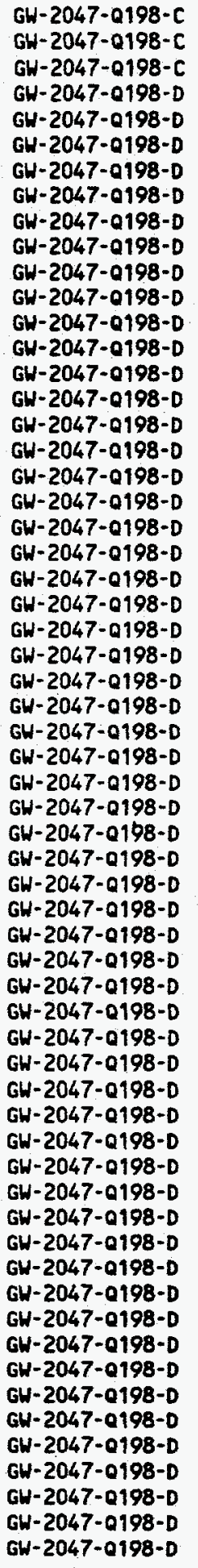 & $\begin{array}{l}01 / 23 / 98 \\
01 / 23 / 98 \\
01 / 23 / 98 \\
01 / 26 / 98 \\
01 / 26 / 98 \\
01 / 26 / 98 \\
01 / 26 / 98 \\
01 / 26 / 98 \\
01 / 26 / 98 \\
01 / 26 / 98 \\
01 / 26 / 98 \\
01 / 26 / 98 \\
01 / 26 / 98 \\
01 / 26 / 98 \\
01 / 26 / 98 \\
01 / 26 / 98 \\
01 / 26 / 98 \\
01 / 26 / 98 \\
01 / 26 / 98 \\
01 / 26 / 98 \\
01 / 26 / 98 \\
01 / 26 / 98 \\
01 / 26 / 98 \\
01 / 26 / 98 \\
01 / 26 / 98 \\
01 / 26 / 98 \\
01 / 26 / 98 \\
01 / 26 / 98 \\
01 / 26 / 98 \\
01 / 26 / 98 \\
01 / 26 / 98 \\
01 / 26 / 98 \\
01 / 26 / 98 \\
01 / 26 / 98 \\
01 / 26 / 98 \\
01 / 26 / 98 \\
01 / 26 / 98 \\
01 / 26 / 98 \\
01 / 26 / 98 \\
01 / 26 / 98 \\
01 / 26 / 98 \\
01 / 26 / 98 \\
01 / 26 / 98 \\
01 / 26 / 98 \\
01 / 26 / 98 \\
01 / 26 / 98 \\
01 / 26 / 98 \\
01 / 26 / 98 \\
01 / 26 / 98 \\
01 / 26 / 98 \\
01 / 26 / 98 \\
01 / 26 / 98 \\
01 / 26 / 98 \\
01 / 26 / 98 \\
01 / 26 / 98 \\
01 / 26 / 98 \\
01 / 26 / 98 \\
01 / 26 / 98 \\
01 / 26 / 98 \\
01 / 26 / 98 \\
01 / 26 / 98\end{array}$ & 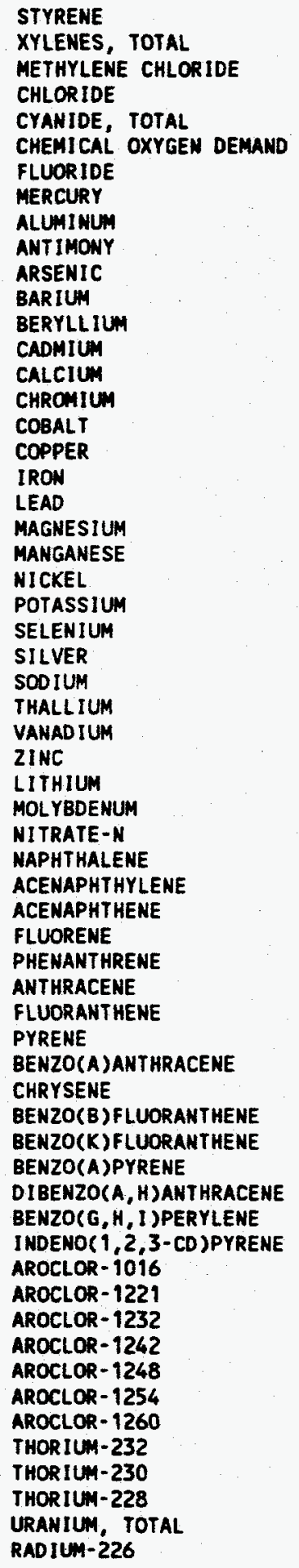 & 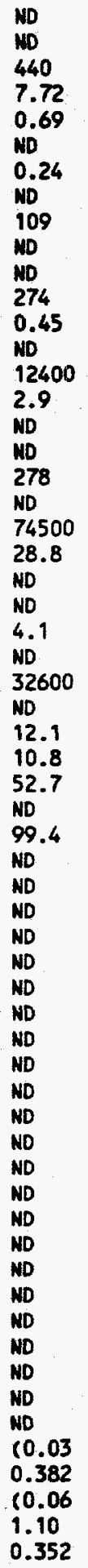 & $\begin{array}{l}10 \\
10 \\
50 \\
0.40 \\
0.55 \\
5.00 \\
0.20 \\
0.10 \\
31.3 \\
42.5 \\
1.3 \\
0.40 \\
0.40 \\
3.3 \\
15.9 \\
2.7 \\
4.3 \\
4.1 \\
5.9 \\
0.90 \\
54.5 \\
1.0 \\
14.6 \\
2180 \\
2.2 \\
6.0 \\
34.4 \\
1.8 \\
2.9 \\
2.3 \\
4.1 \\
11.1 \\
25.0 \\
5.0 \\
5.0 \\
5.0 \\
5.0 \\
5.0 \\
5.0 \\
5.0 \\
5.0 \\
5.0 \\
5.0 \\
5.0 \\
5.0 \\
5.0 \\
5.0 \\
5.0 \\
5.0 \\
1.0 \\
1.0 \\
1.0 \\
1.0 \\
1.0 \\
1.0 \\
1.0 \\
0.098 \\
0.079 \\
0.113 \\
0.677 \\
0.082\end{array}$ & 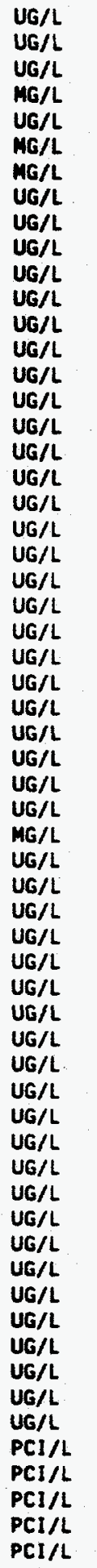 & $\begin{array}{l}\text { EPA CLP } \\
\text { EPA CLP } \\
\text { EPA CLP } \\
\text { EPA } 300.0 \\
\text { EPA CLP } \\
\text { EPA } 410.4 \\
\text { EPA } 300.0 \\
\text { EPA CLP } \\
\text { EPA CLP } \\
\text { EPA CLP } \\
\text { EPA CLP } \\
\text { EPA CLP } \\
\text { EPA CLP } \\
\text { EPA CLP } \\
\text { EPA CLP } \\
\text { EPA CLP } \\
\text { EPA CLP } \\
\text { EPA CLP } \\
\text { EPA CLP } \\
\text { EPA CLP } \\
\text { EPA CLP } \\
\text { EPA CLP } \\
\text { EPA CLP } \\
\text { EPA CLP } \\
\text { EPA CLP } \\
\text { EPA CLP } \\
\text { EPA CLP } \\
\text { EPA CLP } \\
\text { EPA CLP } \\
\text { EPA CLP } \\
\text { EPA CLP } \\
\text { EPA CLP } \\
\text { EPA } 353.1 \\
\text { EPA } 8310 \\
\text { EPA } 8310 \\
\text { EPA } 8310 \\
\text { EPA } 8310 \\
\text { EPA } 8310 \\
\text { EPA } 8310 \\
\text { EPA } 8310 \\
\text { EPA } 8310 \\
\text { EPA } 8310 \\
\text { EPA } 8310 \\
\text { EPA } 8310 \\
\text { EPA } 8310 \\
\text { EPA } 8310 \\
\text { EPA } 8310 \\
\text { EPA } 8310 \\
\text { EPA } 8310 \\
\text { EPA } 8080 A \\
\text { EPA } 8080 A \\
\text { EPA } 8080 A \\
\text { EPA } 8080 A \\
\text { EPA } 8080 A \\
\text { EPA } 8080 A \\
\text { EPA } 8080 A \\
\text { NAS-NS-3004 } \\
\text { NAS-NS-3004 } \\
\text { NAS-NS-3004 } \\
\text { ASTM } 5174-91 \\
\text { EPA } 904.0 \\
\end{array}$ \\
\hline
\end{tabular}




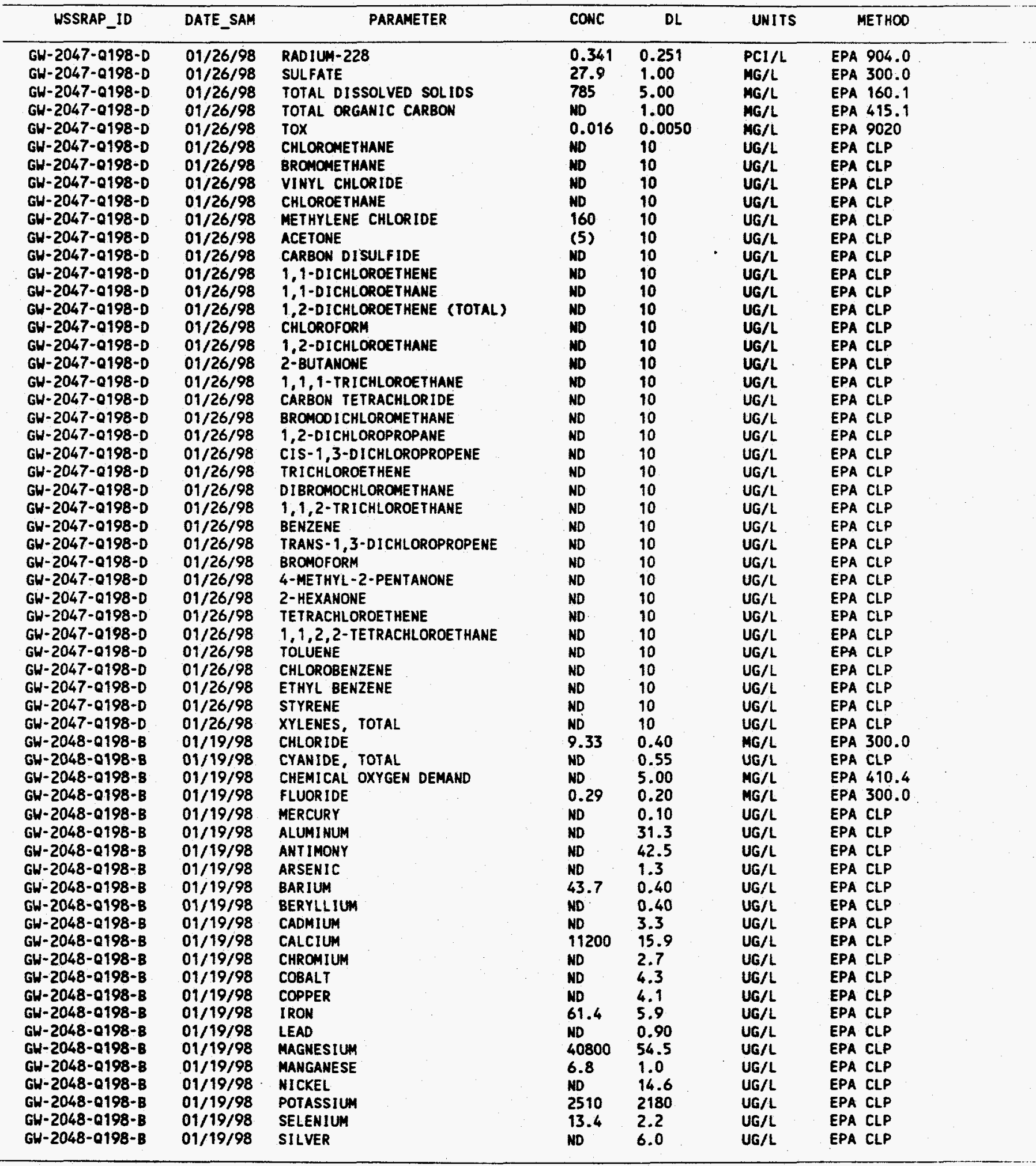




\begin{tabular}{|c|c|c|c|c|c|c|}
\hline WSSRAP_ID & DATE_SAM & PARAMETER & conc & DL & UNITS & METHOO \\
\hline 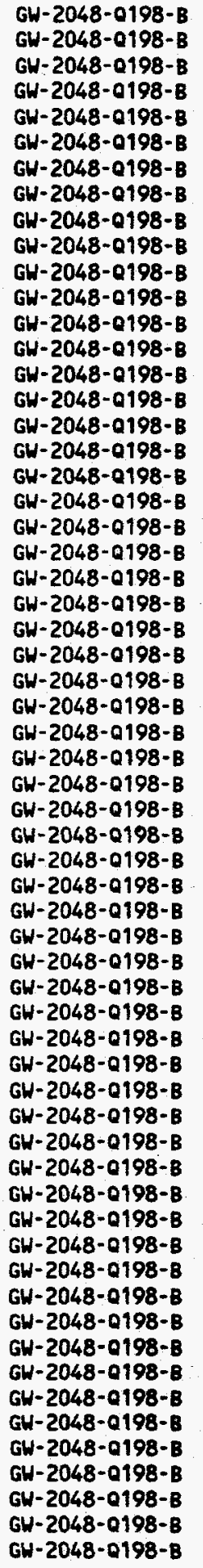 & 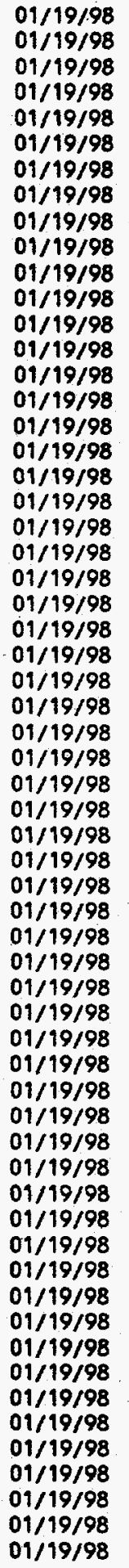 & 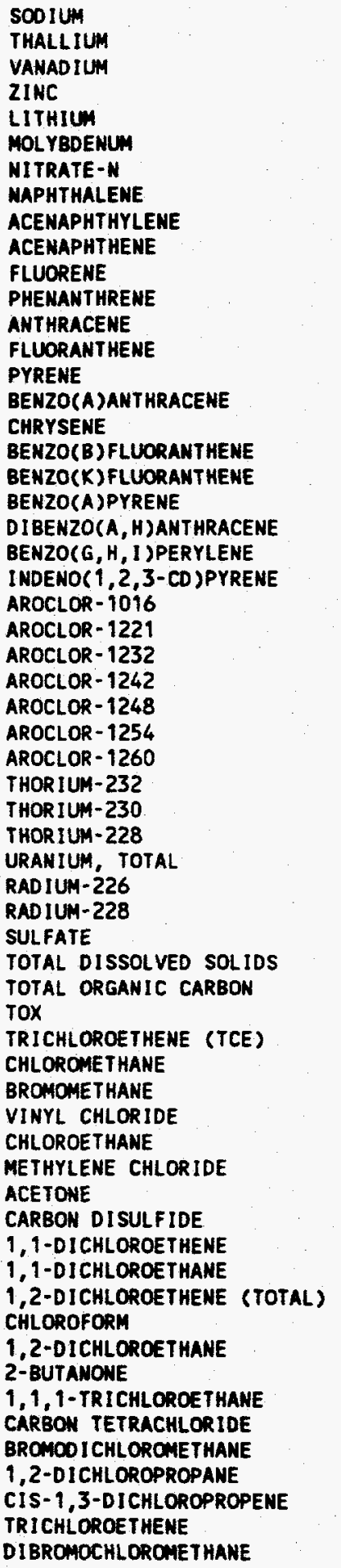 & $\begin{array}{l}71000 \\
\text { ND } \\
9.8 \\
8.7 \\
\text { ND } \\
\text { ND } \\
\text { O.92 } \\
\text { ND } \\
\text { ND } \\
\text { HD } \\
\text { ND } \\
\text { ND } \\
\text { ND } \\
\text { ND } \\
\text { ND } \\
\text { ND } \\
\text { ND } \\
\text { ND } \\
\text { ND } \\
\text { ND } \\
\text { ND } \\
\text { ND } \\
\text { ND } \\
\text { ND } \\
\text { ND } \\
\text { ND } \\
\text { ND } \\
\text { ND } \\
\text { ND } \\
\text { ND } \\
\text { CO.05 } \\
\text { C.13 } \\
\text { ND } \\
1.12 \\
O .045 \\
\text { C.10 } \\
210 \\
609 \\
2.08 \\
0.009 \\
\text { ND } \\
\text { ND } \\
\text { ND } \\
\text { ND } \\
\text { ND } \\
(2) \\
(4) \\
\text { ND } \\
\text { ND } \\
\text { ND } \\
\text { ND } \\
\text { ND } \\
\text { ND } \\
\text { C2) } \\
\text { ND } \\
\text { ND } \\
\text { ND } \\
\text { ND } \\
\text { ND } \\
\text { ND } \\
\text { ND }\end{array}$ & 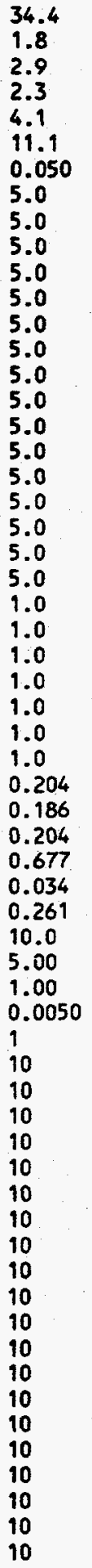 & $\begin{array}{l}U G / L \\
U G / L \\
U G / L \\
U G / L \\
U G / L \\
U G / L \\
U G / L \\
U G / L \\
U G / L \\
U G / L \\
U G / L \\
U G / L \\
U G / L \\
U G / L \\
U G / L \\
U G / L \\
U G / L \\
U G / L \\
U G / L \\
U G / L \\
U G / L \\
U G / L \\
U G / L \\
U G / L \\
U G / L \\
U G / L \\
U G / L \\
U G / L \\
U G / L \\
U G / L \\
P C I / L \\
P C I / L \\
P C I / L \\
P C I / L \\
P C I / L \\
P C I / L \\
M G / L \\
\text { MG/L } \\
M G / L \\
M G / L \\
U G / L \\
U G / L \\
U G / L \\
U G / L \\
U G / L \\
U G / L \\
U G / L \\
U G / L \\
U G / L \\
U G / L \\
U G / L \\
U G / L \\
U G / L \\
U G / L \\
U G / L \\
U G / L \\
U G / L \\
U G / L \\
U G / L \\
U G / L \\
U G / L\end{array}$ & $\begin{array}{l}\text { EPA CLP } \\
\text { EPA CLP } \\
\text { EPA CLP } \\
\text { EPA CLP } \\
\text { EPA CLP } \\
\text { EPA CLP } \\
\text { EPA } 353.1 \\
\text { EPA } 8310 \\
\text { EPA } 8310 \\
\text { EPA } 8310 \\
\text { EPA } 8310 \\
\text { EPA } 8310 \\
\text { EPA } 8310 \\
\text { EPA } 8310 \\
\text { EPA } 8310 \\
\text { EPA } 8310 \\
\text { EPA } 8310 \\
\text { EPA } 8310 \\
\text { EPA } 8310 \\
\text { EPA } 8310 \\
\text { EPA } 8310 \\
\text { EPA } 8310 \\
\text { EPA } 8310 \\
\text { EPA } 8080 A \\
\text { EPA } 8080 A \\
\text { EPA } 8080 A \\
\text { EPA } 8080 A \\
\text { EPA } 8080 A \\
\text { EPA } 8080 A \\
\text { EPA } 8080 A \\
\text { NAS-NS-3004 } \\
\text { NAS-NS-3004 } \\
\text { NAS }-N S-3004 \\
\text { ASTM } 5174-91 \\
\text { EPA } 904.0 \\
\text { EPA } 904.0 \\
\text { EPA } 300.0 \\
\text { EPA } 160.1 \\
\text { EPA } 415.1 \\
\text { EPA } 9020 \\
\text { EPA } 8260 \\
\text { EPA CLP } \\
\text { EPA CLP } \\
\text { EPA CLP } \\
\text { EPA CLP } \\
\text { EPA CLP } \\
\text { EPA CLP } \\
\text { EPA CLP } \\
\text { EPA CLP } \\
\text { EPA CLP } \\
\text { EPA CLP } \\
\text { EPA CLP } \\
\text { EPA CLP } \\
\text { EPA CLP } \\
\text { EPA CLP } \\
\text { EPA CLP } \\
\text { EPA CLP } \\
\text { EPA CLP } \\
\text { EPA CLP } \\
\text { EPA CLP } \\
\text { EPA CLP } \\
\text { CPA }\end{array}$ \\
\hline
\end{tabular}




\begin{tabular}{|c|c|c|c|c|c|c|}
\hline USSRAP_ID & DATE_SAM & PARAMETER & CONC & DL & UNITS & METHOO \\
\hline 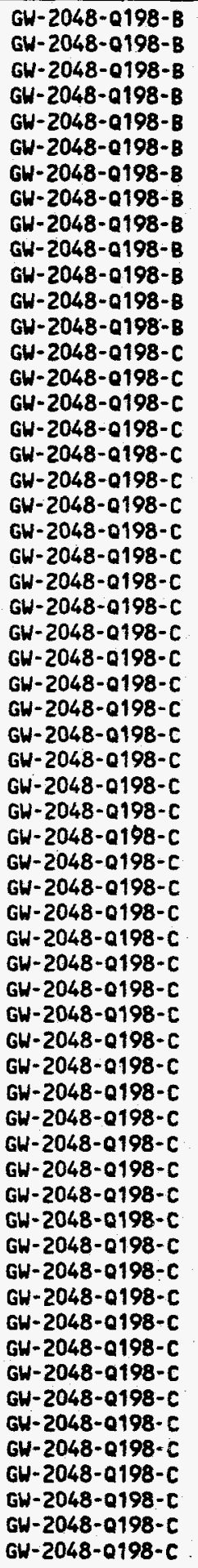 & $\begin{array}{l}01 / 19 / 98 \\
01 / 19 / 98 \\
01 / 19 / 98 \\
01 / 19 / 98 \\
01 / 19 / 98 \\
01 / 19 / 98 \\
01 / 19 / 98 \\
01 / 19 / 98 \\
01 / 19 / 98 \\
01 / 19 / 98 \\
01 / 19 / 98 \\
01 / 19 / 98 \\
01 / 19 / 98 \\
01 / 19 / 98 \\
01 / 19 / 98 \\
01 / 19 / 98 \\
01 / 19 / 98 \\
01 / 19 / 98 \\
01 / 19 / 98 \\
01 / 19 / 98 \\
01 / 19 / 98 \\
01 / 19 / 98 \\
01 / 19 / 98 \\
01 / 19 / 98 \\
01 / 19 / 98 \\
01 / 19 / 98 \\
01 / 19 / 98 \\
01 / 19 / 98 \\
01 / 19 / 98 \\
01 / 19 / 98 \\
01119 / 98 \\
01 / 19 / 98 \\
01 / 19 / 98 \\
01 / 19 / 98 \\
01 / 19 / 98 \\
01 / 19 / 98 \\
01 / 19 / 98 \\
01 / 19 / 98 \\
01 / 19 / 98 \\
01 / 19 / 98 \\
01 / 19 / 98 \\
01 / 19 / 98 \\
01 / 19 / 98 \\
01 / 19 / 98 \\
01 / 19 / 98 \\
011119 / 98 \\
011 / 19 / 98 \\
01 / 19 / 98 \\
01 / 19 / 98 \\
01 / 19 / 98 \\
01 / 19 / 98 \\
01119 / 98 \\
01 / 19 / 98 \\
01 / 19 / 98 \\
01 / 19 / 98 \\
01 / 19 / 98 \\
011 / 19 / 98 \\
01 / 19 / 98 \\
01 / 19 / 98 \\
01 / 19 / 98 \\
01 / 19 / 98\end{array}$ & 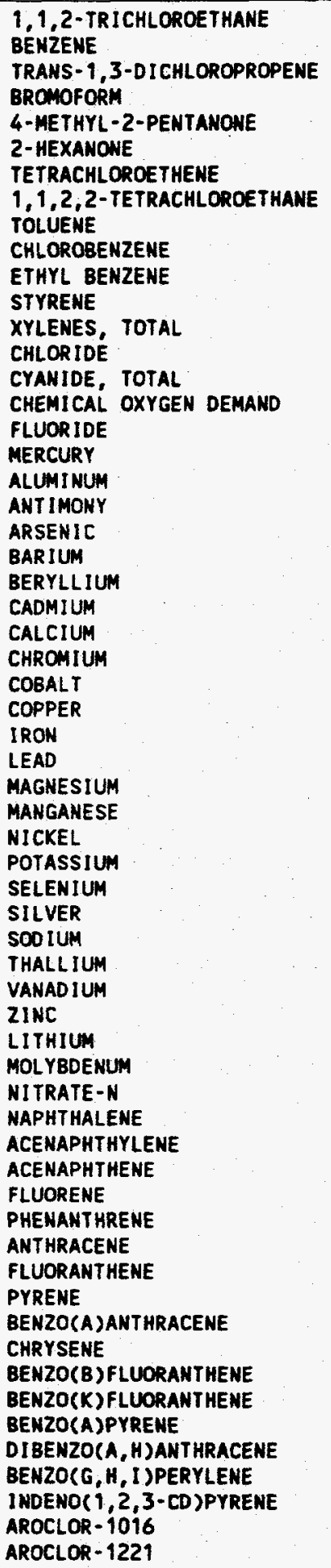 & $\begin{array}{l}\text { ND } \\
\text { ND } \\
\text { ND } \\
\text { ND } \\
\text { ND } \\
\text { ND } \\
\text { ND } \\
\text { ND } \\
\text { ND } \\
\text { ND } \\
\text { ND } \\
\text { ND } \\
\text { HD } \\
8.48 \\
\text { ND } \\
\text { ND } \\
0.28 \\
\text { ND } \\
\text { ND } \\
\text { ND } \\
\text { ND } \\
\text { C2.2 } \\
\text { ND } \\
\text { ND } \\
\text { I0800 } \\
\text { ND } \\
\text { ND } \\
\text { ND } \\
67.3 \\
\text { ND } \\
39300 \\
6.0 \\
\text { ND } \\
3050 \\
10.7 \\
\text { ND } \\
68200 \\
\text { ND } \\
11.1 \\
13.0 \\
4.7 \\
11.3 \\
0.91 \\
\text { ND } \\
\text { ND } \\
\text { ND } \\
\text { ND } \\
\text { ND } \\
\text { ND } \\
\text { ND } \\
\text { ND } \\
\text { ND } \\
\text { ND } \\
\text { ND } \\
\text { ND } \\
\text { ND } \\
\text { ND } \\
\text { ND } \\
\text { ND } \\
\text { ND }\end{array}$ & 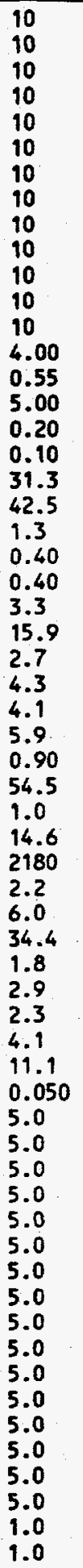 & $\begin{array}{l}\text { UG/L } \\
\text { UG/L } \\
\text { UG/L } \\
\text { UG/L } \\
\text { UG/L } \\
\text { UG/L } \\
\text { UG/L } \\
\text { UG/L } \\
\text { UG/L } \\
\text { UG/L } \\
\text { UG/L } \\
U G / L \\
U G / L \\
\text { UG/L } \\
U G / L \\
\text { UG/L } \\
\text { UG/L } \\
U G / L \\
U G / L \\
U G / L \\
U G / L \\
U G / L \\
U G / L \\
U G / L \\
U G / L \\
U G / L \\
U G / L \\
U G / L \\
U G / L \\
U G / L \\
U G / L \\
U G / L \\
U G / L \\
U G / L \\
U G / L \\
U G / L \\
U G / L \\
U G / L \\
U G / L \\
U G / L \\
U G / L \\
U G / L \\
\text { UG/L } \\
U G / L \\
U G / L \\
U G / L \\
U G / L \\
U G / L \\
U G / L \\
U G / L \\
U G / L \\
U G / L \\
U G / L \\
U G / L \\
U G / L \\
U G / L \\
U G / L \\
U G / L \\
U G / L \\
U G / L \\
U G / L\end{array}$ & 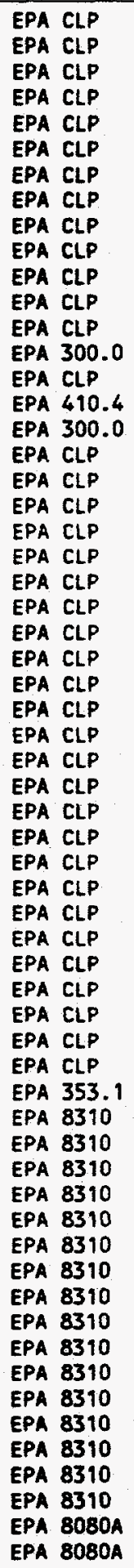 \\
\hline
\end{tabular}




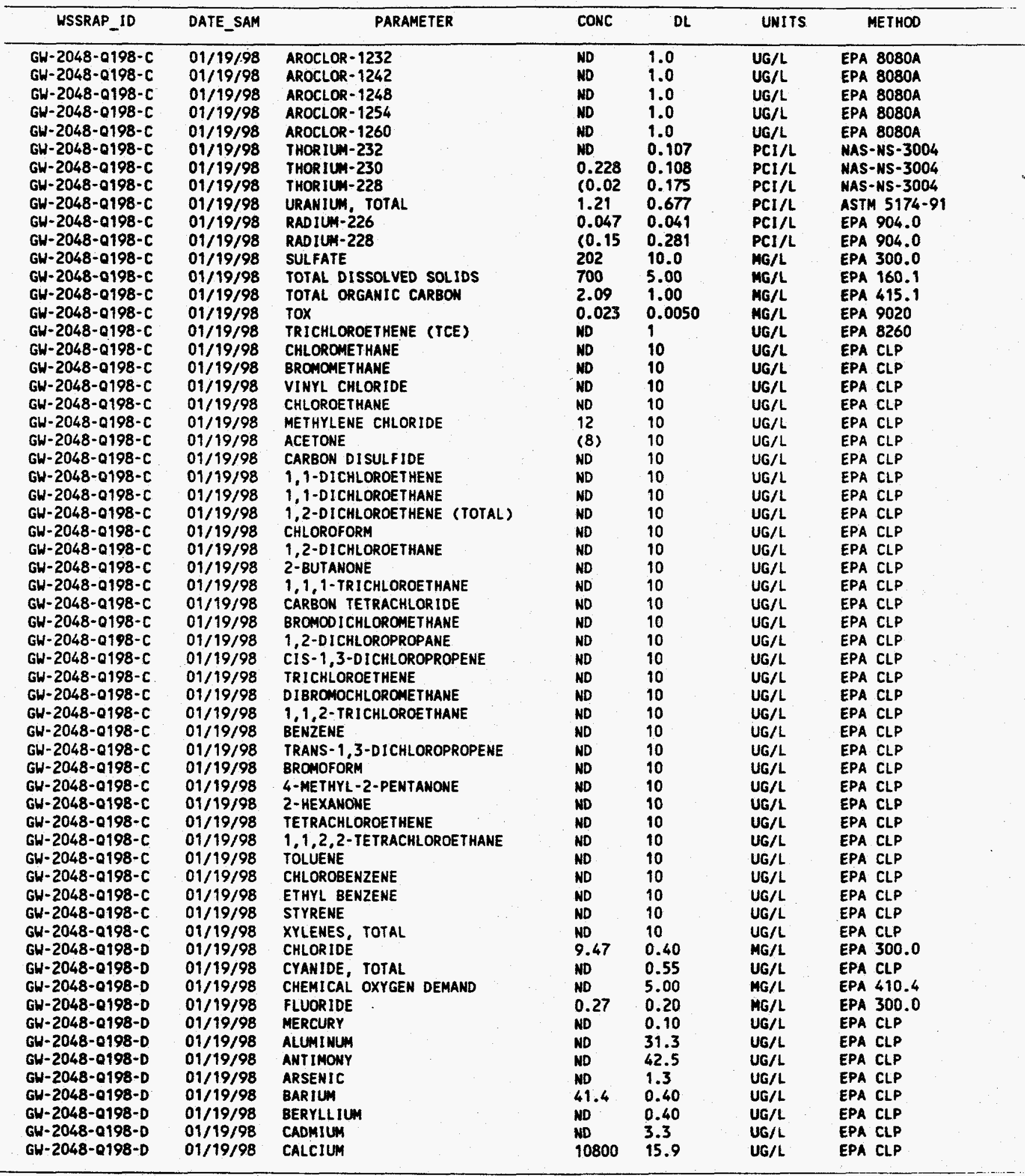




\begin{tabular}{|c|c|c|c|c|c|c|}
\hline WSSRAP_ID & DATE_SAM & PARAMETER & CONC & $D L$ & UNITS & METHOD \\
\hline 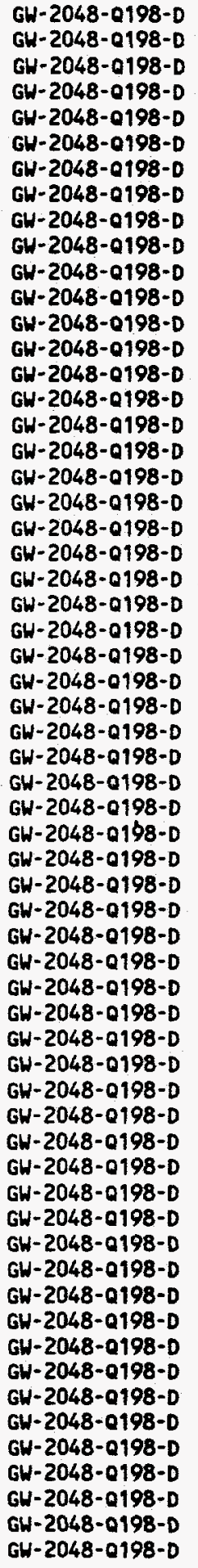 & $\begin{array}{l}01 / 19 / 98 \\
01 / 19 / 98 \\
01 / 19 / 98 \\
01 / 19 / 98 \\
01 / 19 / 98 \\
01 / 19 / 98 \\
01 / 19 / 98 \\
01 / 19 / 98 \\
01 / 19 / 98 \\
01 / 19 / 98 \\
01 / 19 / 98 \\
01 / 19 / 98 \\
01 / 19 / 98 \\
01 / 19 / 98 \\
01 / 19 / 98 \\
01 / 19 / 98 \\
01 / 19 / 98 \\
01 / 19 / 98 \\
01 / 19 / 98 \\
01 / 19 / 98 \\
01 / 19 / 98 \\
01 / 19 / 98 \\
01 / 19 / 98 \\
01 / 19 / 98 \\
01 / 19 / 98 \\
01 / 19 / 98 \\
01 / 19 / 98 \\
01 / 19 / 98 \\
01119 / 98 \\
01 / 19 / 98 \\
01 / 19 / 98 \\
01 / 19 / 98 \\
01 / 19 / 98 \\
01 / 19 / 98 \\
01 / 19 / 98 \\
01 / 19 / 98 \\
01 / 19 / 98 \\
01 / 19 / 98 \\
011 / 19 / 98 \\
01 / 19 / 98 \\
01 / 19 / 98 \\
01 / 19 / 98 \\
011 / 19 / 98 \\
011 / 19 / 98 \\
01 / 19 / 98 \\
01 / 19 / 98 \\
01 / 19 / 98 \\
01119 / 98 \\
011 / 19 / 98 \\
01 / 19 / 98 \\
01 / 19 / 98 \\
01 / 19 / 98 \\
01119 / 98 \\
01 / 19 / 98 \\
01 / 19 / 98 \\
01 / 19 / 98 \\
01 / 19 / 98 \\
01 / 19 / 98 \\
011 / 19 / 98 \\
01 / 19 / 98 \\
01 / 19 / 98\end{array}$ & 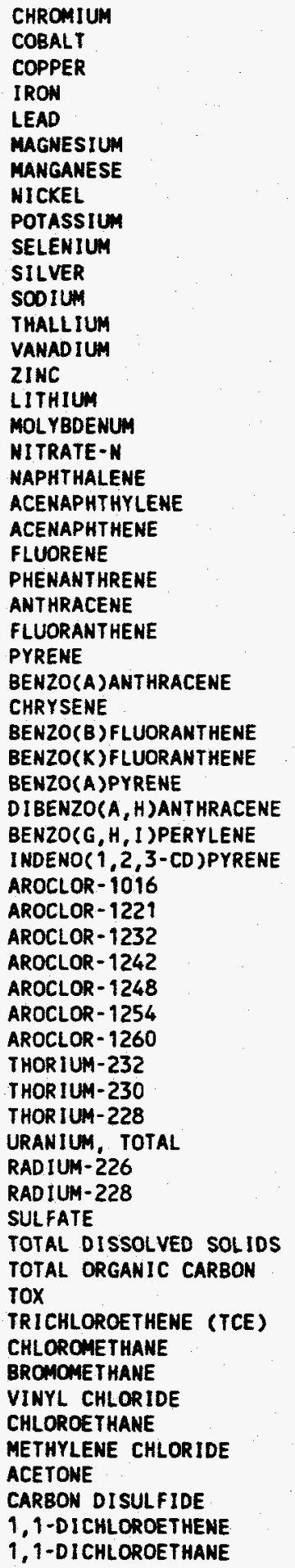 & 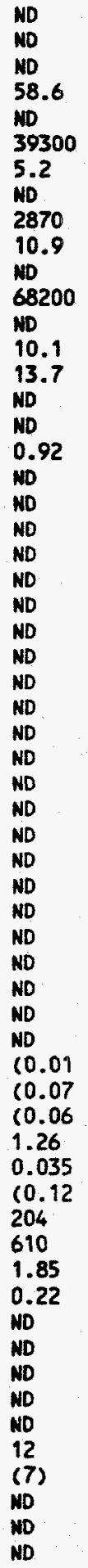 & 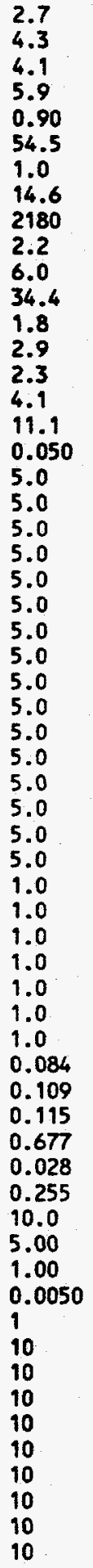 & 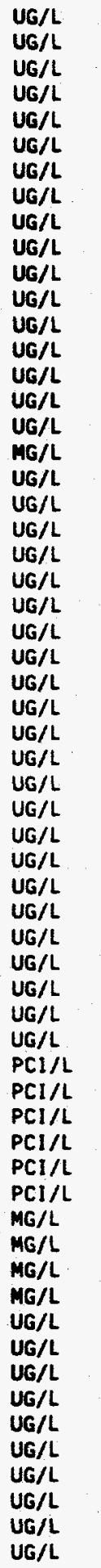 & $\begin{array}{l}\text { EPA CLP } \\
\text { EPA CLP } \\
\text { EPA CLP } \\
\text { EPA CLP } \\
\text { EPA CLP } \\
\text { EPA CLP } \\
\text { EPA CLP } \\
\text { EPA CLP } \\
\text { EPA CLP } \\
\text { EPA CLP } \\
\text { EPA CLP } \\
\text { EPA CLP } \\
\text { EPA CLP } \\
\text { EPA CLP } \\
\text { EPA CLP } \\
\text { EPA CLP } \\
\text { EPA CLP } \\
\text { EPA } 353.1 \\
\text { EPA } 8310 \\
\text { EPA } 8310 \\
\text { EPA } 8310 \\
\text { EPA } 8310 \\
\text { EPA } 8310 \\
\text { EPA } 8310 \\
\text { EPA } 8310 \\
\text { EPA } 8310 \\
\text { EPA } 8310 \\
\text { EPA } 8310 \\
\text { EPA } 8310 \\
\text { EPA } 8310 \\
\text { EPA } 8310 \\
\text { EPA } 8310 \\
\text { EPA } 8310 \\
\text { EPA } 8310 \\
\text { EPA } 8080 A \\
\text { EPA } 8080 A \\
\text { EPA } 8080 A \\
\text { EPA } 8080 A \\
\text { EPA } 8080 A \\
\text { EPA } 8080 A \\
\text { EPA } 8080 A \\
\text { NAS-NS-3004 } \\
\text { NAS-NS-3004 } \\
\text { NAS-NS-3004 } \\
\text { ASTM } 5174-91 \\
\text { EPA } 904.0 \\
\text { EPA } 904.0 \\
\text { EPA } 300.0 \\
\text { EPA } 160.1 \\
\text { EPA } 415.1 \\
\text { EPA } 9020 \\
\text { EPA } 8260 \\
\text { EPA CLP } \\
\text { EPA CLP } \\
\text { EPA CLP } \\
\text { EPA CLP } \\
\text { EPA CLP } \\
\text { EPA CLP } \\
\text { EPA CLP } \\
\text { EPA CLP } \\
\text { EPA CLP } \\
\end{array}$ \\
\hline
\end{tabular}




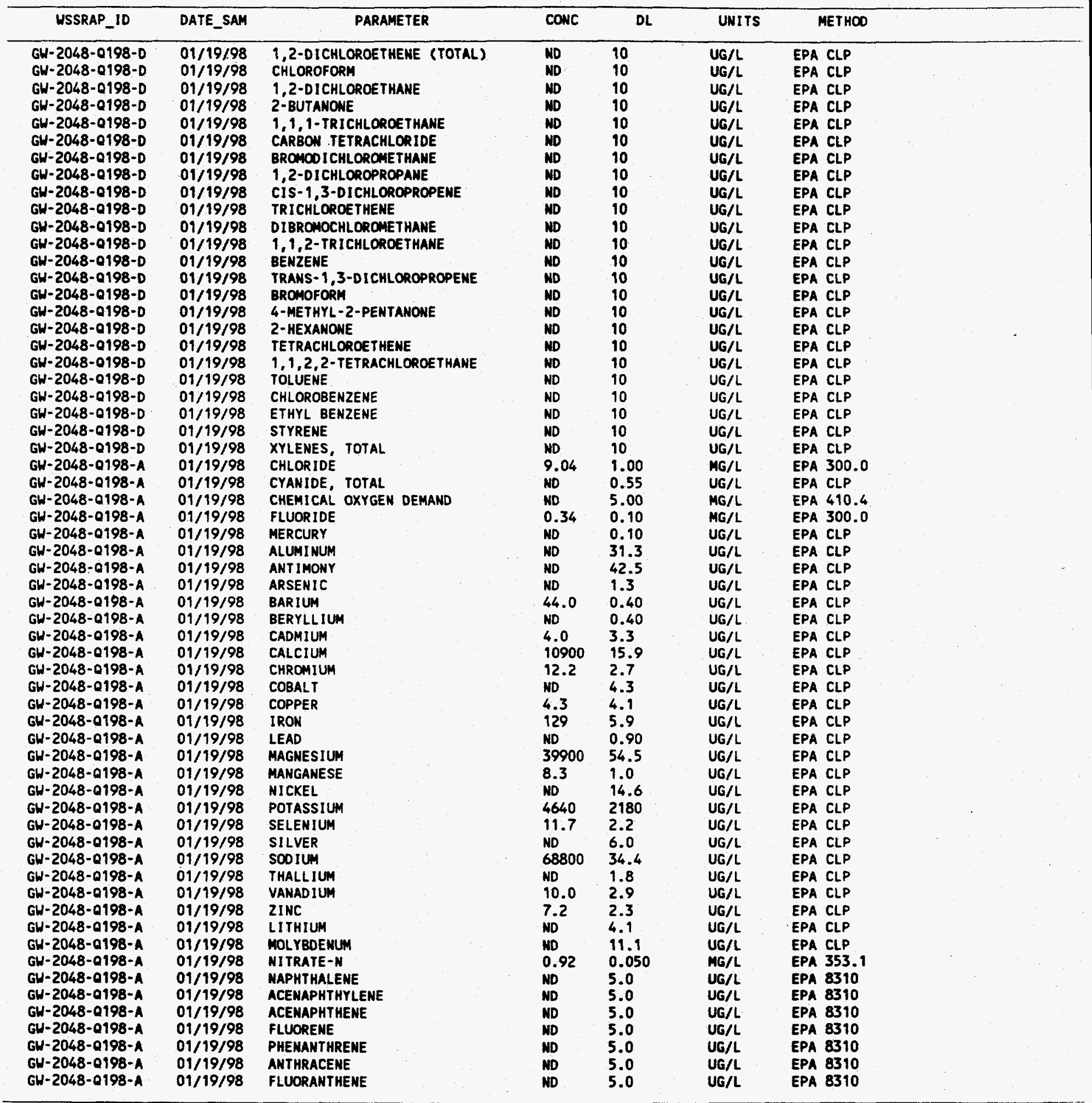




\begin{tabular}{|c|c|c|c|c|c|c|}
\hline WSSRAP_ID & DATE_SAM & PARAMETER & CONC & DL & UNITS & METHOD \\
\hline $\begin{array}{l}G W-2048-0198-A \\
G W-2048-0198-A \\
G W-2048-0198-A \\
G H-2048-0198-A \\
G W-2048-0198-A \\
G W-2048-0198-A \\
G W-2048-0198-A \\
G W-2048-0198-A \\
G W-2048-0198-A \\
G W-2048-0198-A \\
G W-2048-0198-A \\
G W-2048-0198-A \\
G W-2048-0198-A \\
G W-2048-0198-A \\
G W-2048-0198-A \\
G W-2048-0198-A \\
G W-2048-0198-A \\
G W-2048-0198-A \\
G W-2048-0198-A \\
G W-2048-0198-A \\
G W-2048-0198-A \\
G W-2048-0198-A \\
G W-2048-0198-A \\
G W-2048-0198-A \\
G W-2048-0198-A \\
G W-2048-0198-A \\
G W-2048-0198-A \\
G W-2048-0198-A \\
G W-2048-0198-A \\
G W-2048-0198-A \\
G W-2048-0198-A \\
G W-2048-0198-A \\
G W-2048-0198-A \\
G W-2048-0198-A \\
G W-2048-0198-A \\
G W-2048-0198-A \\
G W-2048-0198-A \\
G H-2048-0198-A \\
G W-2048-0198-A \\
G W-2048-0198-A \\
G W-2048-0198-A \\
G W-2048-0198-A \\
G W-2048-0198-A \\
G W-2048-0198-A \\
G W-2048-0198-A \\
G W-2048-0198-A \\
G W-2048-0198-A \\
G W-2048-0198-A \\
G W-2048-0198-A \\
G W-2048-0198-A \\
G W-2048-0198-A \\
G W-2048-01988-A \\
G W-2048-0198-A \\
G W-2048-0198-A \\
G W-2048-0198-A \\
G W-2048-0198-A \\
G W-2048-0198-A \\
G W-2048-019198-A \\
G W-2048-0198-A \\
G W-2048-0198-A \\
G W-3003-0997\end{array}$ & $\begin{array}{l}01 / 19 / 98 \\
01 / 19 / 98 \\
01 / 19 / 98 \\
01 / 19 / 98 \\
01 / 19 / 98 \\
01 / 19 / 98 \\
01 / 19 / 98 \\
01 / 19 / 98 \\
01 / 19 / 98 \\
01 / 19 / 98 \\
01 / 19 / 98 \\
01 / 19 / 98 \\
01 / 19 / 98 \\
01 / 19 / 98 \\
01 / 19 / 98 \\
01 / 19 / 98 \\
01 / 19 / 98 \\
01 / 19 / 98 \\
01 / 19 / 98 \\
01 / 19 / 98 \\
01 / 19 / 98 \\
01 / 19 / 98 \\
01 / 19 / 98 \\
011 / 19 / 98 \\
01 / 19 / 98 \\
01 / 19 / 98 \\
01 / 19 / 98 \\
01 / 19 / 98 \\
01 / 19 / 98 \\
01119 / 98 \\
01 / 19 / 98 \\
01 / 19 / 98 \\
01 / 19 / 98 \\
01 / 19 / 98 \\
01 / 19 / 98 \\
01 / 19 / 98 \\
01 / 19 / 98 \\
01 / 19 / 98 \\
01 / 19 / 98 \\
01119 / 98 \\
01 / 19 / 98 \\
01 / 19 / 98 \\
01 / 19 / 98 \\
01 / 19 / 98 \\
01 / 19 / 98 \\
01 / 19 / 98 \\
01 / 19 / 98 \\
01 / 19 / 98 \\
01 / 19 / 98 \\
01 / 19 / 98 \\
01 / 19 / 98 \\
01 / 19 / 98 \\
01119 / 98 \\
01119 / 98 \\
01 / 19 / 98 \\
01 / 19 / 98 \\
01 / 19 / 98 \\
01 / 19 / 98 \\
01 / 19 / 98 \\
01 / 19 / 98 \\
09 / 09 / 97\end{array}$ & 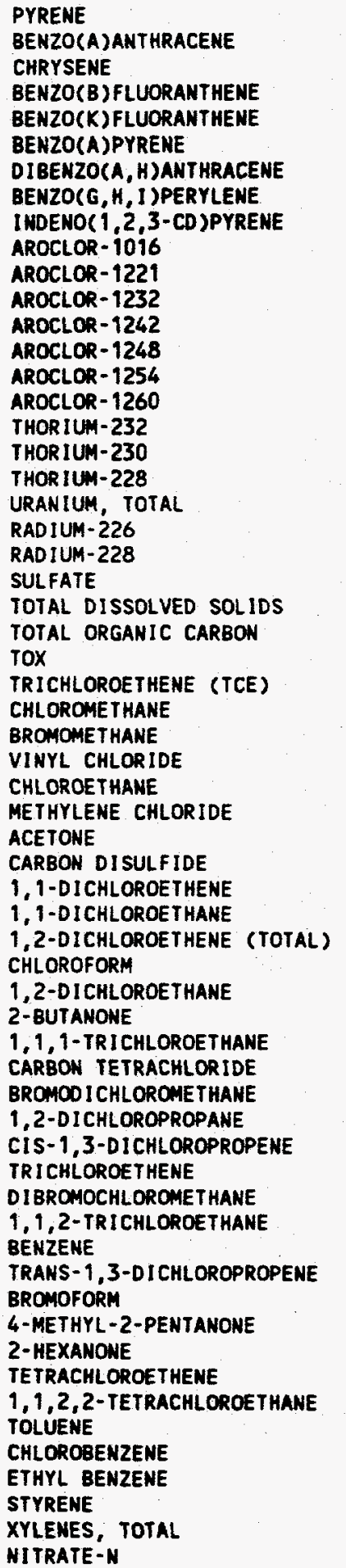 & $\begin{array}{l}\text { ND } \\
\text { ND } \\
\text { ND } \\
\text { ND } \\
\text { ND } \\
\text { ND } \\
\text { ND } \\
\text { ND } \\
\text { ND } \\
\text { ND } \\
\text { ND } \\
\text { ND } \\
\text { ND } \\
\text { ND } \\
\text { ND } \\
0.028 \\
O .145 \\
O .112 \\
1.26 \\
O .074 \\
C 0.05 \\
210 \\
\text { O49 } \\
2.20 \\
O .017 \\
\text { ND } \\
\text { ND } \\
\text { ND } \\
\text { ND } \\
\text { ND } \\
\text { N1 } \\
\text { C7) } \\
\text { ND } \\
\text { ND } \\
\text { ND } \\
\text { ND } \\
\text { ND } \\
\text { ND } \\
\text { ND } \\
\text { ND } \\
\text { ND } \\
\text { ND } \\
\text { ND } \\
\text { ND } \\
\text { ND } \\
\text { ND } \\
\text { ND } \\
\text { ND } \\
\text { ND } \\
\text { ND } \\
\text { ND } \\
\text { ND } \\
\text { ND } \\
\text { ND } \\
\text { ND } \\
\text { ND } \\
\text { ND } \\
\text { 370 }\end{array}$ & $\begin{array}{l}5.0 \\
5.0 \\
5.0 \\
5.0 \\
5.0 \\
5.0 \\
5.0 \\
5.0 \\
5.0 \\
1.0 \\
1.0 \\
1.0 \\
1.0 \\
1.0 \\
1.0 \\
1.0 \\
0.02 \\
0.07 \\
0.108 \\
0\end{array}$ & $\begin{array}{l}U G / L \\
U G / L \\
U G / L \\
U G / L \\
U G / L \\
U G / L \\
U G / L \\
U G / L \\
U G / L \\
U G / L \\
U G / L \\
U G / L \\
U G / L \\
U G / L \\
U G / L \\
U G / L \\
P C I / L \\
P C I / L \\
P C I / L \\
P C I / L \\
P C I / L \\
P C I / L \\
M G / L \\
M G / L \\
M G / L \\
M G / L \\
U G / L \\
U G / L \\
U G / L \\
U G / L \\
U G / L \\
U G / L \\
U G / L \\
U G / L \\
U G / L \\
U G / L \\
U G / L \\
U G / L \\
U G / L \\
U G / L \\
U G / L \\
U G / L \\
U G / L \\
U G / L \\
U G / L \\
U G / L \\
U G / L \\
U G / L \\
U G / L \\
U G / L \\
U G / L \\
U G / L \\
U G / L \\
U G / L \\
U G / L \\
U\end{array}$ & $\begin{array}{l}\text { EPA } 8310 \\
\text { EPA } 8310 \\
\text { EPA } 8310 \\
\text { EPA } 8310 \\
\text { EPA } 8310 \\
\text { EPA } 8310 \\
\text { EPA } 8310 \\
\text { EPA } 8310 \\
\text { EPA } 8310 \\
\text { EPA } 8080 A \\
\text { EPA } 8080 A \\
\text { EPA } 8080 A \\
\text { EPA } 8080 A \\
\text { EPA } 8080 A \\
\text { EPA } 8080 A \\
\text { EPA } 8080 A \\
\text { NAS-NS-3004 } \\
\text { NAS-NS-3004 } \\
\text { NAS-NS-3004 } \\
\text { ASTM } 5174-91 \\
\text { EPA } 904.0 \\
\text { EPA } 904.0 \\
\text { EPA } 300.0 \\
\text { EPA } 160.1 \\
\text { EPA } 415.1 \\
\text { EPA } 9020 \\
\text { EPA } 8260 \\
\text { EPA CLP } \\
\text { EPA CLP } \\
\text { EPA CLP } \\
\text { EPA CLP } \\
\text { EPA CLP } \\
\text { EPA CLP } \\
\text { EPA CLP } \\
\text { EPA CLP } \\
\text { EPA CLP } \\
\text { EPA CLP } \\
\text { EPA CLP } \\
\text { EPA CLP } \\
\text { EPA CLP } \\
\text { EPA CLP } \\
\text { EPA CLP } \\
\text { EPA CLP } \\
\text { EPA CLP } \\
\text { EPA CLP } \\
\text { EPA CLP } \\
\text { EPA CLP } \\
\text { EPA CLP } \\
\text { EPA CLP } \\
\text { EPA CLP } \\
\text { EPA CLP } \\
\text { EPA CLP } \\
\text { EPA CLP } \\
\text { EPA CLP } \\
\text { EPA CLP } \\
\text { EPA CLP } \\
\text { EPA CLP } \\
\text { EPA CLP } \\
\text { EPA CLP } \\
\text { EPA CLP } \\
\text { EPA } 353.1\end{array}$ \\
\hline
\end{tabular}




\begin{tabular}{|c|c|c|c|c|c|c|}
\hline USSRAP_ID & DATE_SAM & PARAMETER & CONC & DL & UNITS & METHOO \\
\hline 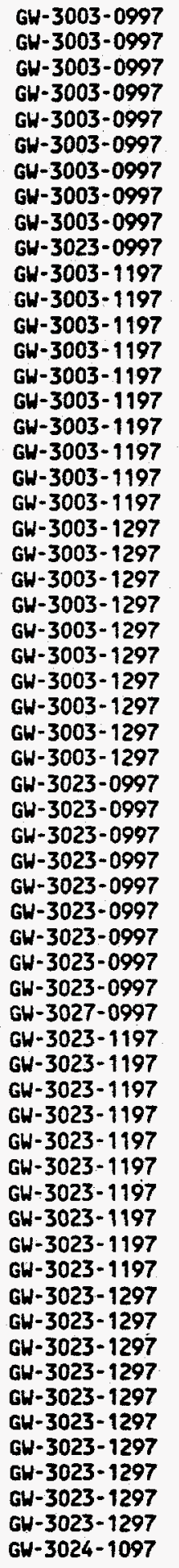 & $\begin{array}{l}09 / 09 / 97 \\
09 / 09 / 97 \\
09 / 09 / 97 \\
09 / 09 / 97 \\
09 / 09 / 97 \\
09 / 09 / 97 \\
09 / 09 / 97 \\
09 / 09 / 97 \\
09 / 09 / 97 \\
09 / 09 / 97 \\
11 / 03 / 97 \\
11 / 03 / 97 \\
11 / 03 / 97 \\
11 / 03 / 97 \\
11 / 03 / 97 \\
11 / 03 / 97 \\
11 / 03 / 97 \\
11 / 03 / 97 \\
11 / 03 / 97 \\
11 / 03 / 97 \\
12 / 01 / 97 \\
12 / 01 / 97 \\
12 / 01 / 97 \\
12 / 01 / 97 \\
12 / 01 / 97 \\
12 / 01 / 97 \\
12 / 01 / 97 \\
12 / 01 / 97 \\
12 / 01 / 97 \\
12 / 01 / 97 \\
09 / 09 / 97 \\
09 / 09 / 97 \\
09 / 09 / 97 \\
09 / 09 / 97 \\
09 / 09 / 97 \\
09 / 09 / 97 \\
09 / 09 / 97 \\
09 / 09 / 97 \\
09 / 09 / 97 \\
09 / 09 / 97 \\
11 / 03 / 97 \\
11 / 03 / 97 \\
11 / 03 / 97 \\
11 / 03 / 97 \\
111 / 03 / 97 \\
11 / 03 / 97 \\
11 / 03 / 97 \\
11 / 03 / 97 \\
11103 / 97 \\
111 / 03 / 97 \\
12 / 01 / 97 \\
12 / 011 / 97 \\
12 / 011 / 97 \\
12 / 01 / 97 \\
12 / 01 / 97 \\
12 / 01 / 97 \\
12 / 011 / 97 \\
12 / 011 / 97 \\
12 / 01 / 97 \\
12 / 01 / 97 \\
10 / 13 / 97 \\
\end{array}$ & $\begin{array}{l}\text { SULFATE } \\
\text { ARSENIC } \\
\text { BARIUM } \\
\text { CADMIUM } \\
\text { CHROMIUM } \\
\text { LEAD } \\
\text { MERCURY } \\
\text { SELENIUM } \\
\text { SILVER } \\
\text { SILVER } \\
\text { NITRATE-N } \\
\text { SULFATE } \\
\text { ARSENIC } \\
\text { BARIUM } \\
\text { CADMIUM } \\
\text { CHROMIUM } \\
\text { LEAD } \\
\text { MERCURY } \\
\text { SELENIUM } \\
\text { SILVER } \\
\text { NITRATE-N } \\
\text { SULFATE } \\
\text { ARSENIC } \\
\text { BARIUM } \\
\text { CADMIUM } \\
\text { CHROMIUM } \\
\text { LEAD } \\
\text { MERCURY } \\
\text { SELENIUM } \\
\text { SILVER } \\
\text { NITRATE-N } \\
\text { SULFATE } \\
\text { ARSENIC } \\
\text { BARIUM } \\
\text { CADMIUM } \\
\text { CHROMIUM } \\
\text { LEAD } \\
\text { MERCURY } \\
\text { SELENIUM } \\
\text { SILVER } \\
\text { NIIRATE-N } \\
\text { SULFATE } \\
\text { ARSENIC } \\
\text { BARIUM } \\
\text { CADMIUM } \\
\text { CHROMIUM } \\
\text { LEAD } \\
\text { MERCURY } \\
\text { SELENIUM } \\
\text { SILVER } \\
\text { NITRATE-N } \\
\text { SULFATE } \\
\text { ARSENIC } \\
\text { BARIUHM } \\
\text { CADMIUM } \\
\text { CHROMIUM } \\
\text { LEAD } \\
\text { MERCURY } \\
\text { SELENIUM } \\
\text { SILVER } \\
\text { NITRATE-N }\end{array}$ & $\begin{array}{l}162 \\
\text { ND } \\
189 \\
\text { ND } \\
2.9 \\
4.7 \\
\text { ND } \\
12.1 \\
3.4 \\
2.7 \\
367 \\
118 \\
\text { ND } \\
200 \\
\text { ND } \\
\text { ND } \\
4.3 \\
\text { ND } \\
13.3 \\
1.3 \\
362 \\
142 \\
\text { ND } \\
183 \\
\text { ND } \\
\text { ND } \\
\text { ND } \\
\text { ND } \\
11.2 \\
\text { ND } \\
158 \\
196 \\
\text { ND } \\
57.5 \\
\text { ND } \\
9.9 \\
6.8 \\
1.4 \\
11.2 \\
3.1 \\
148 \\
172 \\
\text { ND } \\
54.2 \\
\text { ND } \\
7.6 \\
4.9 \\
1.3 \\
7.9 \\
\text { ND } \\
133 \\
160 \\
\text { ND } \\
60.4 \\
2.9 \\
10.7 \\
4.8 \\
1.4 \\
9.3 \\
1.0 \\
461\end{array}$ & $\begin{array}{l}1.80 \\
2.5 \\
0.2 \\
0.3 \\
0.5 \\
1.2 \\
0.1 \\
2.3 \\
0.6 \\
0.6 \\
10.0 \\
12.5 \\
4.0 \\
2.0 \\
2.0 \\
2.0 \\
2.0 \\
0.10 \\
5.0 \\
1.0 \\
10.0 \\
12.5 \\
4.0 \\
2.0 \\
2.0 \\
2.0 \\
2.0 \\
0.10 \\
5.0 \\
1.0 \\
0.560 \\
1.80 \\
2.5 \\
0.2 \\
0.3 \\
0.5 \\
1.2 \\
0.1 \\
2.3 \\
0.6 \\
5.00 \\
12.5 \\
4.0 \\
2.0 \\
2.0 \\
2.0 \\
2.0 \\
0.10 \\
5.0 \\
1.0 \\
10.0 \\
12.5 \\
4.0 \\
2.0 \\
2.0 \\
2.0 \\
2.0 \\
0.10 \\
5.0 \\
1.0 \\
10.0 \\
\end{array}$ & $\begin{array}{l}\text { MG/L } \\
U G / L \\
U G / L \\
U G / L \\
U G / L \\
U G / L \\
U G / L \\
U G / L \\
U G / L \\
U G / L \\
M G / L \\
\text { MG/L } \\
U G / L \\
U G / L \\
U G / L \\
U G / L \\
U G / L \\
U G / L \\
U G / L \\
U G / L \\
M G / L \\
M G / L \\
U G / L \\
U G / L \\
U G / L \\
U G / L \\
U G / L \\
U G / L \\
U G / L \\
U G / L \\
M G / L \\
M G / L \\
U G / L \\
U G / L \\
U G / L \\
U G / L \\
U G / L \\
U G / L \\
U G / L \\
U G / L \\
M G / L \\
\text { U. } \\
U G / L \\
U G / L \\
U G / L \\
U G / L \\
U G / L \\
U G / L \\
U G / L \\
U G / L \\
U G / L \\
U G / L \\
U G / L \\
M G / L \\
M G / L \\
U G / L \\
U G / L \\
U G / L \\
U G / L \\
U G / L \\
\text { US } / L\end{array}$ & $\begin{array}{l}\text { EPA } 300.0 \\
\text { EPA } 6010 A \\
\text { EPA } 6010 A \\
\text { EPA } 6010 A \\
\text { EPA } 6010 A \\
\text { EPA } 6010 A \\
\text { EPA7470A } \\
\text { EPA } 6010 A \\
\text { EPA } 6010 A \\
\text { EPA } 6010 A \\
\text { EPA } 353.1 \\
\text { EPA } 300.0 \\
\text { EPA } 6010 A \\
\text { EPA } 6010 A \\
\text { EPA } 6010 A \\
\text { EPA } 6010 A \\
\text { EPA } 6010 A \\
\text { EPA } 7470 A \\
\text { EPA } 6010 A \\
\text { EPA } 6010 A \\
\text { EPA } 353.1 \\
\text { EPA } 300.0 \\
\text { EPA CLP } \\
\text { EPA } C L P \\
\text { EPA CLP } \\
\text { EPA CLP } \\
\text { EPA CLP } \\
\text { EPA } C L P \\
\text { EPA CLP } \\
\text { EPA CLP } \\
\text { EPA } 353.1 \\
\text { EPA } 300.0 \\
\text { EPA } 6010 A \\
\text { EPA } 6010 A \\
\text { EPA } 6010 A \\
\text { EPA } 6010 A \\
\text { EPA } 6010 A \\
\text { EPA7470A } \\
\text { EPA } 6010 A \\
\text { EPA } 6010 A \\
\text { EPA } 353.1 \\
\text { EPA } 300.0 \\
\text { EPA } 6010 A \\
\text { EPA } 6010 A \\
\text { EPA } 6010 A \\
\text { EPA } 6010 A \\
\text { EPA } 6010 A \\
\text { EPA } 7470 A \\
\text { EPA } 6010 A \\
\text { EPA } 6010 A \\
\text { EPA } 353.1 \\
\text { EPA } 300.0 \\
\text { EPA } \text { CLP } \\
\text { EPA CLP } \\
\text { EPA CLP } \\
\text { EPA } \text { CLP } \\
\text { EPA CLP } \\
\text { EPA CLP } \\
\text { EPA CLP } \\
\text { EPA CLP } \\
\text { EPA } 353.1\end{array}$ \\
\hline
\end{tabular}




\begin{tabular}{|c|c|c|c|c|c|c|}
\hline WSSRAP_ID & DATE_SAM & PARAMETER & CONC & $D L$ & UNITS & METHOO \\
\hline 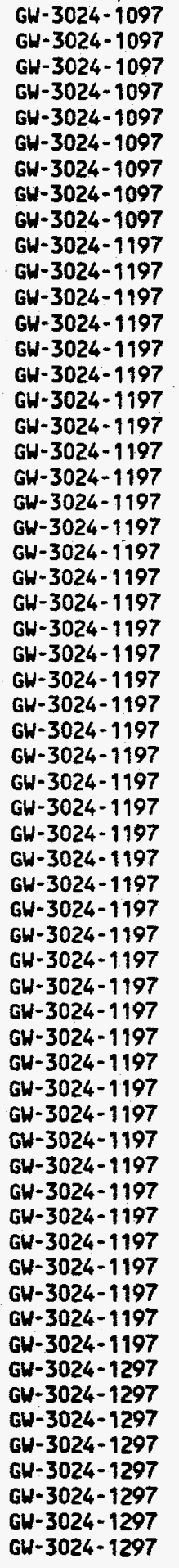 & $\begin{array}{l}10 / 13 / 97 \\
10 / 13 / 97 \\
10 / 13 / 97 \\
10 / 13 / 97 \\
10 / 13 / 97 \\
10 / 13 / 97 \\
10 / 13 / 97 \\
10 / 13 / 97 \\
10 / 13 / 97 \\
11 / 04 / 97 \\
11 / 04 / 97 \\
11 / 04 / 97 \\
11 / 04 / 97 \\
11 / 04 / 97 \\
11 / 04 / 97 \\
11 / 04 / 97 \\
11 / 04 / 97 \\
11 / 04 / 97 \\
11 / 04 / 97 \\
11 / 04 / 97 \\
11 / 04 / 97 \\
11 / 04 / 97 \\
11 / 04 / 97 \\
11 / 04 / 97 \\
11 / 04 / 97 \\
11 / 04 / 97 \\
11 / 04 / 97 \\
11 / 04 / 97 \\
11104 / 97 \\
11104 / 97 \\
11 / 04 / 97 \\
11 / 04 / 97 \\
11 / 04 / 97 \\
11 / 04 / 97 \\
11 / 04 / 97 \\
11 / 04 / 97 \\
11 / 04 / 97 \\
11 / 04 / 97 \\
11 / 04 / 97 \\
111 / 04 / 97 \\
11 / 04 / 97 \\
11 / 04 / 97 \\
11 / 04 / 97 \\
11 / 04 / 97 \\
111 / 04 / 97 \\
11 / 04 / 97 \\
11 / 04 / 97 \\
11 / 04 / 97 \\
11 / 04 / 97 \\
111 / 04 / 97 \\
11 / 04 / 97 \\
11 / 04 / 97 \\
11 / 04 / 97 \\
12 / 02 / 97 \\
12 / 02 / 97 \\
12 / 02 / 97 \\
12 / 02 / 97 \\
12 / 02 / 97 \\
12 / 02 / 97 \\
12 / 02 / 97 \\
12 / 02 / 97\end{array}$ & 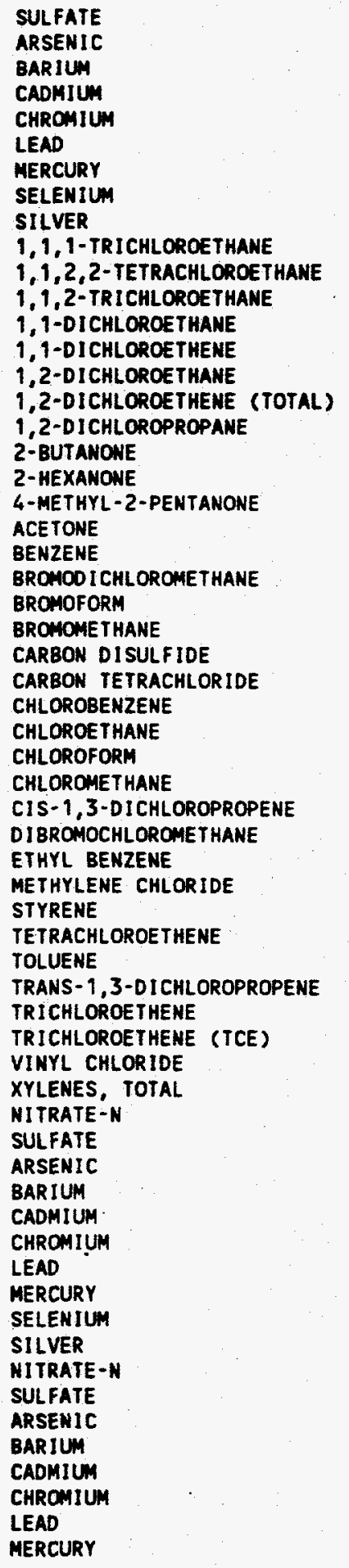 & $\begin{array}{l}69.2 \\
\text { ND } \\
262 \\
\text { ND } \\
3.6 \\
\text { ND } \\
\text { ND } \\
\text { ND } \\
1.2 \\
\text { ND } \\
\text { ND } \\
\text { HD } \\
\text { ND } \\
\text { ND } \\
\text { ND } \\
\text { ND } \\
\text { ND } \\
\text { ND } \\
\text { ND } \\
\text { ND } \\
\text { ND } \\
\text { ND } \\
\text { ND } \\
\text { ND } \\
\text { ND } \\
\text { ND } \\
\text { ND } \\
\text { ND } \\
\text { ND } \\
\text { ND } \\
\text { ND } \\
\text { ND } \\
\text { ND } \\
\text { ND } \\
\text { ND } \\
\text { ND } \\
\text { ND } \\
\text { ND } \\
\text { ND } \\
\text { ND } \\
\text { ND } \\
\text { ND } \\
\text { ND } \\
389 \\
66.1 \\
\text { ND } \\
275 \\
\text { ND } \\
2.7 \\
\text { ND } \\
\text { ND } \\
\text { ND } \\
\text { ND } \\
345 \\
65.9 \\
\text { ND } \\
274 \\
\text { ND } \\
4.3 \\
2.8 \\
\text { ND }\end{array}$ & $\begin{array}{l}5.0 \\
4.0 \\
2.0 \\
2.0 \\
2.0 \\
2.0 \\
0.10 \\
5.0 \\
1.0 \\
10 \\
10 \\
10 \\
10 \\
10 \\
10 \\
10 \\
10 \\
10 \\
10 \\
10 \\
10 \\
10 \\
10 \\
10 \\
10 \\
10 \\
10 \\
10 \\
10 \\
10 \\
10 \\
10 \\
10 \\
10 \\
10 \\
10 \\
10 \\
10 \\
10 \\
10 \\
1.0 \\
10 \\
10 \\
10.0 \\
5.0 \\
4.0 \\
2.0 \\
2.0 \\
2.0 \\
2.0 \\
0.10 \\
5.0 \\
1.0 \\
10.0 \\
5.0 \\
4.0 \\
2.0 \\
2.0 \\
2.0 \\
2.0 \\
0.10\end{array}$ & 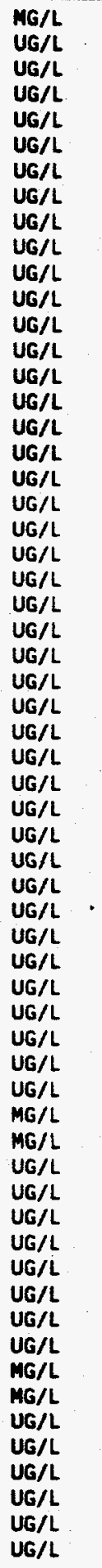 & 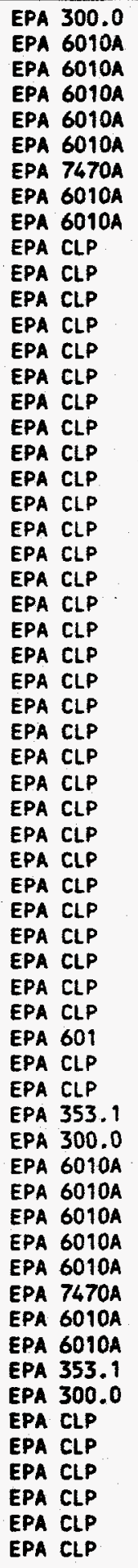 \\
\hline
\end{tabular}




\begin{tabular}{|c|c|c|c|c|c|c|}
\hline WSSRAP_ID & DATE_SAM & PARAMETER & CONC & $D L$ & UNITS & METHOD \\
\hline $\begin{array}{l}\text { GW-3024-1297 } \\
G W-3024-1297 \\
G W-3024-1297 \\
G W-3024-1297 \\
G W-3024-1297 \\
G W-3024-1297 \\
G W-3024-1297 \\
G W-3024-1297 \\
G W-3024-1297 \\
G W-3024-1297 \\
G W-3024-1297 \\
G W-3024-1297 \\
G W-3024-1297 \\
G W-3024-1297 \\
G W-3024-1297 \\
G W-3024-1297 \\
G W-3024-1297 \\
G W-3024-1297 \\
G W-3024-1297 \\
G W-3024-1297 \\
G W-3024-1297 \\
G W-3024-1297 \\
G W-3024-1297 \\
G W-3024-1297 \\
G W-3024-1297 \\
G W-3024-1297 \\
G W-3024-1297 \\
G W-3024-1297 \\
G W-3024-1297 \\
G W-3024-1297 \\
G W-3024-1297 \\
G W-3024-1297 \\
G W-3024-1297 \\
G W-3024-1297 \\
G W-3024-1297 \\
G W-3024-1297 \\
G W-3025-1097 \\
G W-3025-1097 \\
G W-3025-1097 \\
G W-3025-1097 \\
G W-3025-1097 \\
G W-3025-11197 \\
G W-3025-1097 \\
G W-3025-1197 \\
G W-3025-1197 \\
G W-3025-10997 \\
G W-3025-1097 \\
G W-3025-1097 \\
G W-3025-1197 \\
G W-3025-11197 \\
G W-3025-1197 \\
G W-3025-1197 \\
G W-3025-1197 \\
G W-3025-1197 \\
G W-3025-1197 \\
G W-3025-11197 \\
\text { GW-3025-1197 }\end{array}$ & $\begin{array}{l}12 / 02 / 97 \\
12 / 02 / 97 \\
12 / 02 / 97 \\
12 / 02 / 97 \\
12 / 02 / 97 \\
12 / 02 / 97 \\
12 / 02 / 97 \\
12 / 02 / 97 \\
12 / 02 / 97 \\
12 / 02 / 97 \\
12 / 02 / 97 \\
12 / 02 / 97 \\
12 / 02 / 97 \\
12 / 02 / 97 \\
12 / 02 / 97 \\
12 / 02 / 97 \\
12 / 02 / 97 \\
12 / 02 / 97 \\
12 / 02 / 97 \\
12 / 02 / 97 \\
12 / 02 / 97 \\
12 / 02 / 97 \\
12 / 02 / 97 \\
12 / 02 / 97 \\
12 / 02 / 97 \\
12 / 02 / 97 \\
12 / 02 / 97 \\
12 / 02 / 97 \\
12 / 02 / 97 \\
12 / 02 / 97 \\
12 / 02 / 97 \\
12 / 02 / 97 \\
12 / 02 / 97 \\
12 / 02 / 97 \\
12 / 02 / 97 \\
12 / 02 / 97 \\
10 / 13 / 97 \\
10 / 13 / 97 \\
10 / 13 / 97 \\
10 / 13 / 97 \\
10 / 13 / 97 \\
10 / 13 / 97 \\
10 / 13 / 97 \\
10 / 13 / 97 \\
10 / 13 / 97 \\
10 / 13 / 97 \\
11 / 04 / 97 \\
111 / 04 / 97 \\
11 / 04 / 97 \\
11 / 04 / 97 \\
11 / 04 / 97 \\
11 / 04 / 97 \\
111 / 04 / 97 \\
11 / 04 / 97 \\
11 / 04 / 97 \\
11 / 04 / 97 \\
11 / 04 / 97 \\
111 / 04 / 97 \\
11 / 04 / 97 \\
11 / 04 / 97 \\
11 / 04 / 97\end{array}$ & 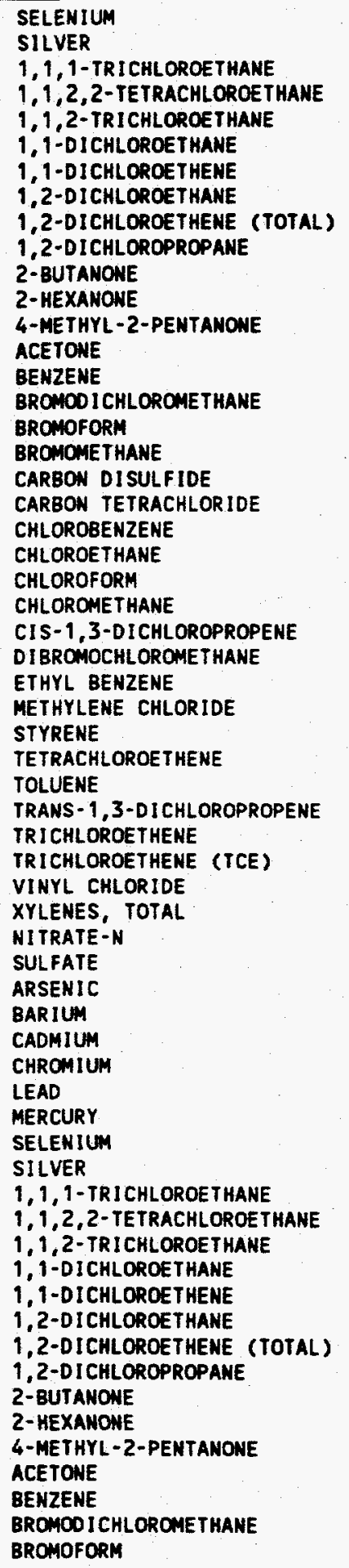 & 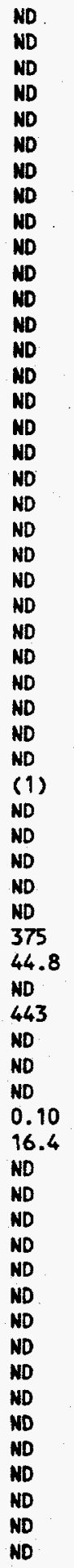 & $\begin{array}{l}5.0 \\
1.0 \\
10 \\
10 \\
10 \\
10 \\
10 \\
10 \\
10 \\
10 \\
10 \\
10 \\
10 \\
10 \\
10 \\
10 \\
10 \\
10 \\
10 \\
10 \\
10 \\
10 \\
10 \\
10 \\
10 \\
10 \\
10 \\
10 \\
10 \\
10 \\
10 \\
10 \\
10 \\
1.0 \\
10 \\
10 \\
10.0 \\
2.5 \\
4.0 \\
2.0 \\
2.0 \\
2.0 \\
2.0 \\
0.10 \\
5.0 \\
1.0 \\
10 \\
10 \\
10 \\
10 \\
10 \\
10 \\
10 \\
10 \\
10 \\
10 \\
10 \\
10 \\
10 \\
10 \\
10\end{array}$ & 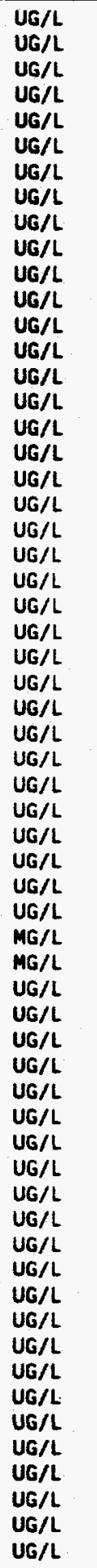 & $\begin{array}{l}\text { EPA CLP } \\
\text { EPA CLP } \\
\text { EPA CLP } \\
\text { EPA CLP } \\
\text { EPA CLP } \\
\text { EPA CLP } \\
\text { EPA CLP } \\
\text { EPA CLP } \\
\text { EPA CLP } \\
\text { EPA CLP } \\
\text { EPA CLP } \\
\text { EPA CLP } \\
\text { EPA CLP } \\
\text { EPA CLP } \\
\text { EPA CLP } \\
\text { EPA CLP } \\
\text { EPA CLP } \\
\text { EPA CLP } \\
\text { EPA CLP } \\
\text { EPA CLP } \\
\text { EPA CLP } \\
\text { EPA CLP } \\
\text { EPA CLP } \\
\text { EPA CLP } \\
\text { EPA CLP } \\
\text { EPA CLP } \\
\text { EPA CLP } \\
\text { EPA CLP } \\
\text { EPA CLP } \\
\text { EPA CLP } \\
\text { EPA CLP } \\
\text { EPA CLP } \\
\text { EPA CLP } \\
\text { EPA } 601 \\
\text { EPA CLP } \\
\text { EPA CLP } \\
\text { EPA } 353.1 \\
\text { EPA } 300.0 \\
\text { EPA } 6010 A \\
\text { EPA } 6010 A \\
\text { EPA } 6010 A \\
\text { EPA } 6010 A \\
\text { EPA } 6010 A \\
\text { EPA } 7470 A \\
\text { EPA } 6010 A \\
\text { EPA } 6010 A \\
\text { EPA CLP } \\
\text { EPA CLP } \\
\text { EPA CLP } \\
\text { EPA CLP } \\
\text { EPA CLP } \\
\text { EPA CLP } \\
\text { EPA CLP } \\
\text { EPA CLP } \\
\text { EPA CLP } \\
\text { EPA CLP } \\
\text { EPA CLP } \\
\text { EPA CLP } \\
\text { EPA CLP } \\
\text { EPA CLP } \\
\text { EPA CLP }\end{array}$ \\
\hline
\end{tabular}




\begin{tabular}{|c|c|c|c|c|c|c|}
\hline WSSRAP_ID & DATE_SAM & PARAMETER & CONC & $\mathrm{DL}$ & UNITS & METHOD \\
\hline 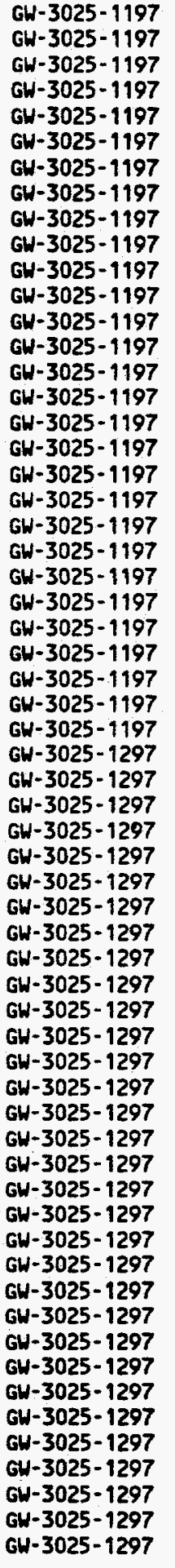 & $\begin{array}{l}11 / 04 / 97 \\
11 / 04 / 97 \\
11 / 04 / 97 \\
11 / 04 / 97 \\
11 / 04 / 97 \\
11 / 04 / 97 \\
11 / 04 / 97 \\
11 / 04 / 97 \\
11 / 04 / 97 \\
11 / 04 / 97 \\
11 / 04 / 97 \\
11 / 04 / 97 \\
11 / 04 / 97 \\
11 / 04 / 97 \\
11 / 04 / 97 \\
11 / 04 / 97 \\
11 / 04 / 97 \\
11 / 04 / 97 \\
11 / 04 / 97 \\
11 / 04 / 97 \\
11 / 04 / 97 \\
11 / 04 / 97 \\
11 / 04 / 97 \\
11 / 04 / 97 \\
11 / 04 / 97 \\
11 / 04 / 97 \\
11 / 04 / 97 \\
11 / 04 / 97 \\
11 / 04 / 97 \\
12 / 02 / 97 \\
12 / 02 / 97 \\
12 / 02 / 97 \\
12 / 02 / 97 \\
12 / 02 / 97 \\
12 / 02 / 97 \\
12 / 02 / 97 \\
12 / 02 / 97 \\
12 / 02 / 97 \\
12 / 02 / 97 \\
12 / 02 / 97 \\
12 / 02 / 97 \\
12 / 02 / 97 \\
12 / 02 / 97 \\
12 / 02 / 97 \\
12 / 02 / 97 \\
12 / 02 / 97 \\
12 / 02 / 97 \\
12 / 02 / 97 \\
12 / 02 / 97 \\
12 / 02 / 97 \\
12 / 02 / 97 \\
12 / 02 / 97 \\
12 / 02 / 97 \\
12 / 02 / 97 \\
12 / 02 / 97 \\
12 / 02 / 97 \\
12 / 02 / 97 \\
12 / 02 / 97 \\
12 / 02 / 97 \\
12 / 02 / 97 \\
12 / 02 / 97\end{array}$ & 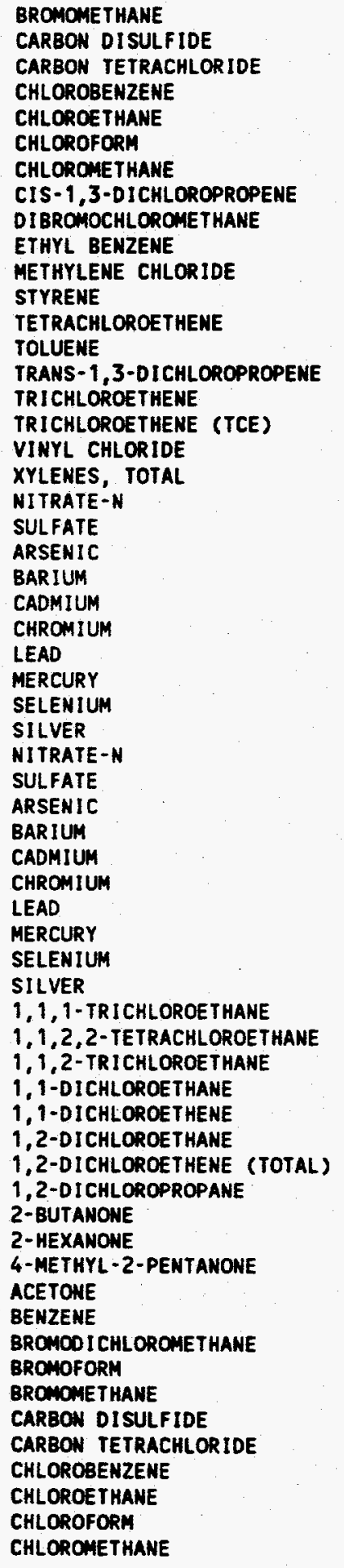 & 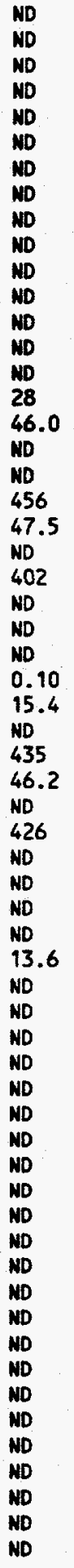 & $\begin{array}{l}10 \\
10 \\
10 \\
10 \\
10 \\
10 \\
10 \\
10 \\
10 \\
10 \\
10 \\
10 \\
10 \\
10 \\
10 \\
10 \\
5.0 \\
10 \\
10 \\
20.0 \\
5.0 \\
4.0 \\
2.0 \\
2.0 \\
2.0 \\
2.0 \\
0.10 \\
5.0 \\
1.0 \\
10.0 \\
2.5 \\
4.0 \\
2.0 \\
2.0 \\
2.0 \\
2.0 \\
0.10 \\
5.0 \\
1.0 \\
10 \\
10 \\
10 \\
10 \\
10 \\
10 \\
10 \\
10 \\
10 \\
10 \\
10 \\
10 \\
10 \\
10 \\
10 \\
10 \\
10 \\
10 \\
10 \\
10 \\
10\end{array}$ & $\begin{array}{l}U G / L \\
U G / L \\
U G / L \\
U G / L \\
U G / L \\
U G / L \\
U G / L \\
U G / L \\
U G / L \\
U G / L \\
U G / L \\
U G / L \\
U G / L \\
U G / L \\
U G / L \\
U G / L \\
U G / L \\
U G / L \\
U G / L \\
U G / L \\
U G / L \\
U G / L \\
U G / L \\
U G / L \\
U G / L \\
U G / L \\
U G / L \\
U G / L \\
U G / L \\
\text { UG/L } \\
M G / L \\
U G / L \\
U G / L \\
U G / L \\
U G / L \\
U G / L \\
U G / L \\
U G / L \\
U G / L \\
U G / L \\
U G / L \\
U G / L \\
U G / L \\
U G / L \\
U G / L \\
U G / L \\
U G / L \\
U G / L \\
U G / L \\
U G / L \\
U G / L \\
U G / L \\
U G / L \\
U G / L \\
U G / L \\
U G / L \\
U G / L \\
U G / L \\
U G / L \\
U\end{array}$ & $\begin{array}{l}\text { EPA CLP } \\
\text { EPA CLP } \\
\text { EPA CLP } \\
\text { EPA CLP } \\
\text { EPA CLP } \\
\text { EPA CLP } \\
\text { EPA CLP } \\
\text { EPA CLP } \\
\text { EPA CLP } \\
\text { EPA CLP } \\
\text { EPA CLP } \\
\text { EPA CLP } \\
\text { EPA CLP } \\
\text { EPA CLP } \\
\text { EPA CLP } \\
\text { EPA CLP } \\
\text { EPA } 601 \\
\text { EPA CLP } \\
\text { EPA CLP } \\
\text { EPA } 353.1 \\
\text { EPA } 300.0 \\
\text { EPA } 6010 A \\
\text { EPA } 6010 A \\
\text { EPA } 6010 A \\
\text { EPA } 6010 A \\
\text { EPA } 6010 A \\
\text { EPA } 7470 A \\
\text { EPA } 6010 A \\
\text { EPA } 6010 A \\
\text { EPA } 353.1 \\
\text { EPA } 300.0 \\
\text { EPA CLP } \\
\text { EPA CLP } \\
\text { EPA CLP } \\
\text { EPA CLP } \\
\text { EPA CLP } \\
\text { EPA CLP } \\
\text { EPA CLP } \\
\text { EPA CLP } \\
\text { EPA CLP } \\
\text { EPA CLP } \\
\text { EPA CLP } \\
\text { EPA CLP } \\
\text { EPA CLP } \\
\text { EPA CLP } \\
\text { EPA CLP } \\
\text { EPA CLP } \\
\text { EPA CLP } \\
\text { EPA CLP } \\
\text { EPA CLP } \\
\text { EPA CLP } \\
\text { EPA CLP } \\
\text { EPA CLP } \\
\text { EPA CLP } \\
\text { EPA CLP } \\
\text { EPA CLP } \\
\text { EPA CLP } \\
\text { EPA CLP } \\
\text { EPA CLP } \\
\text { EPA CLP } \\
\text { EPA CLP }\end{array}$ \\
\hline
\end{tabular}




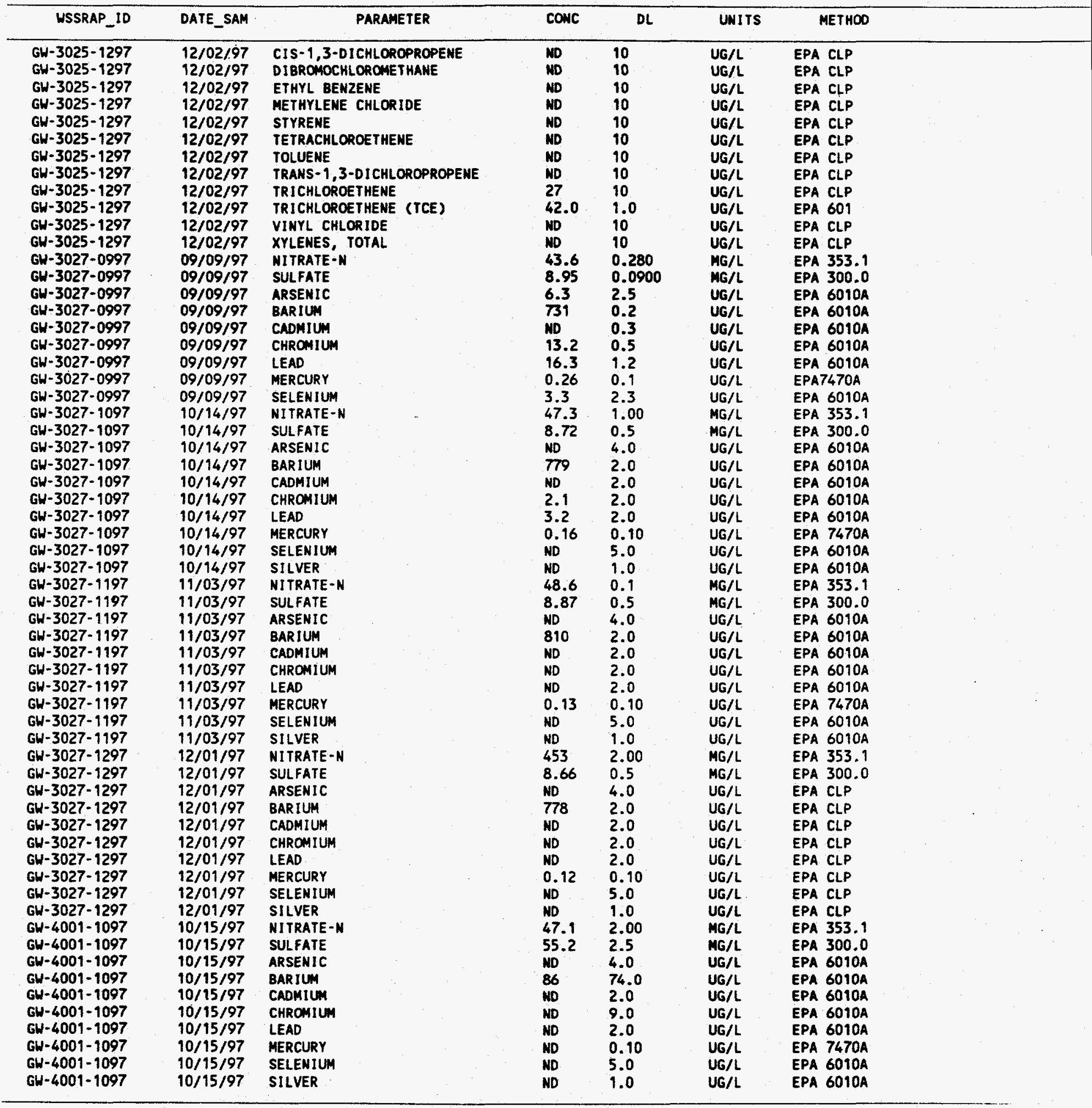




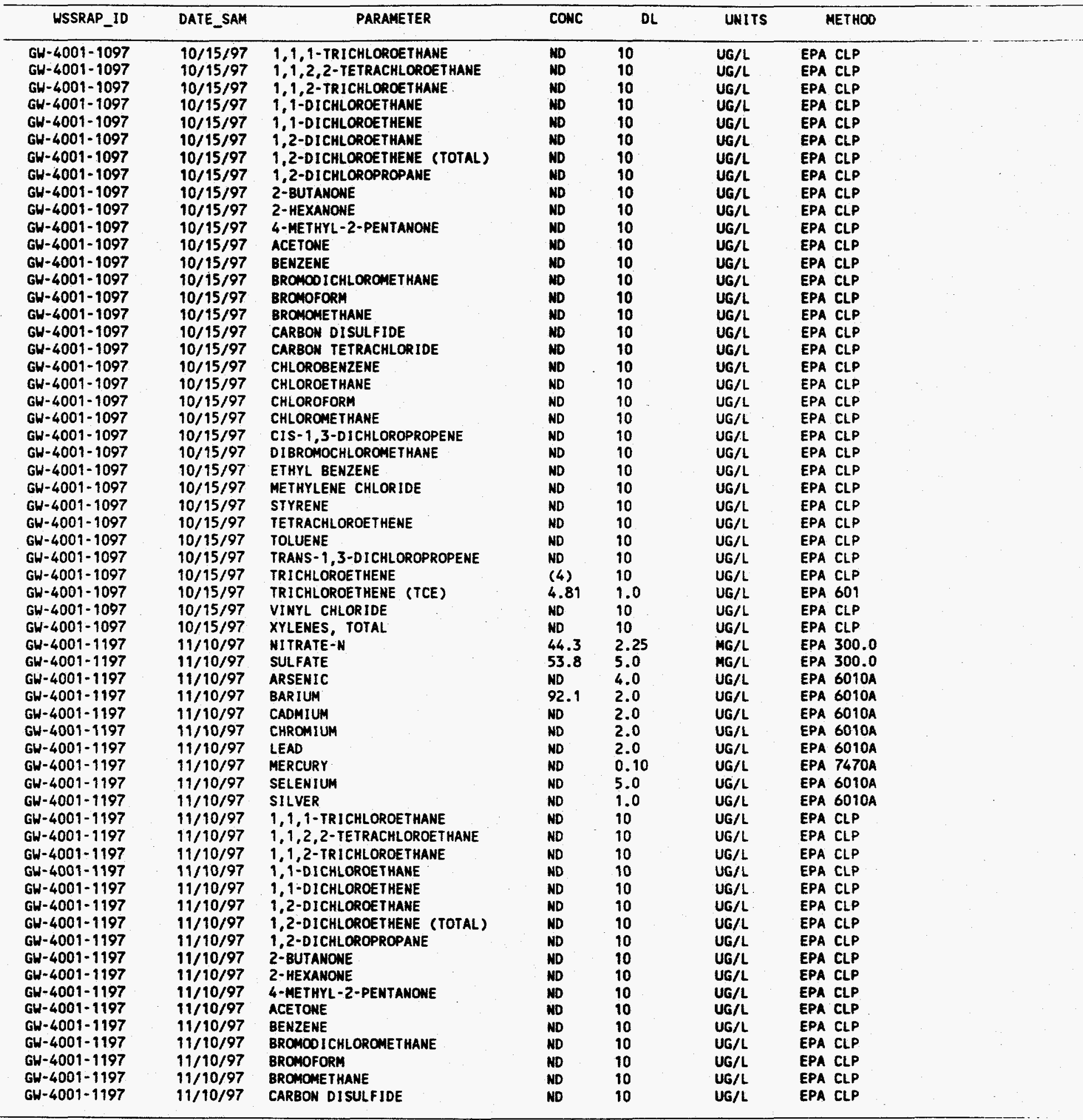




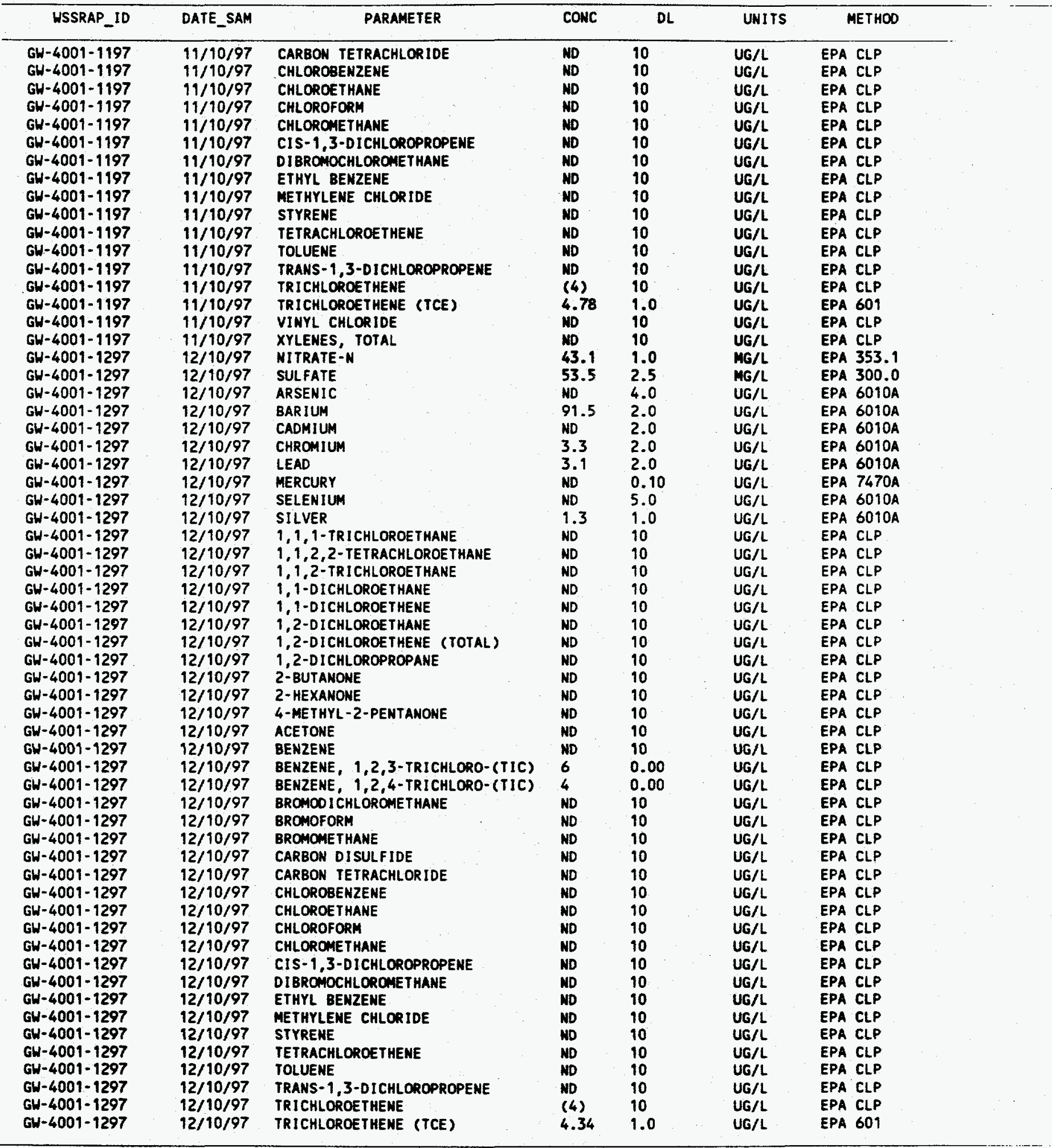




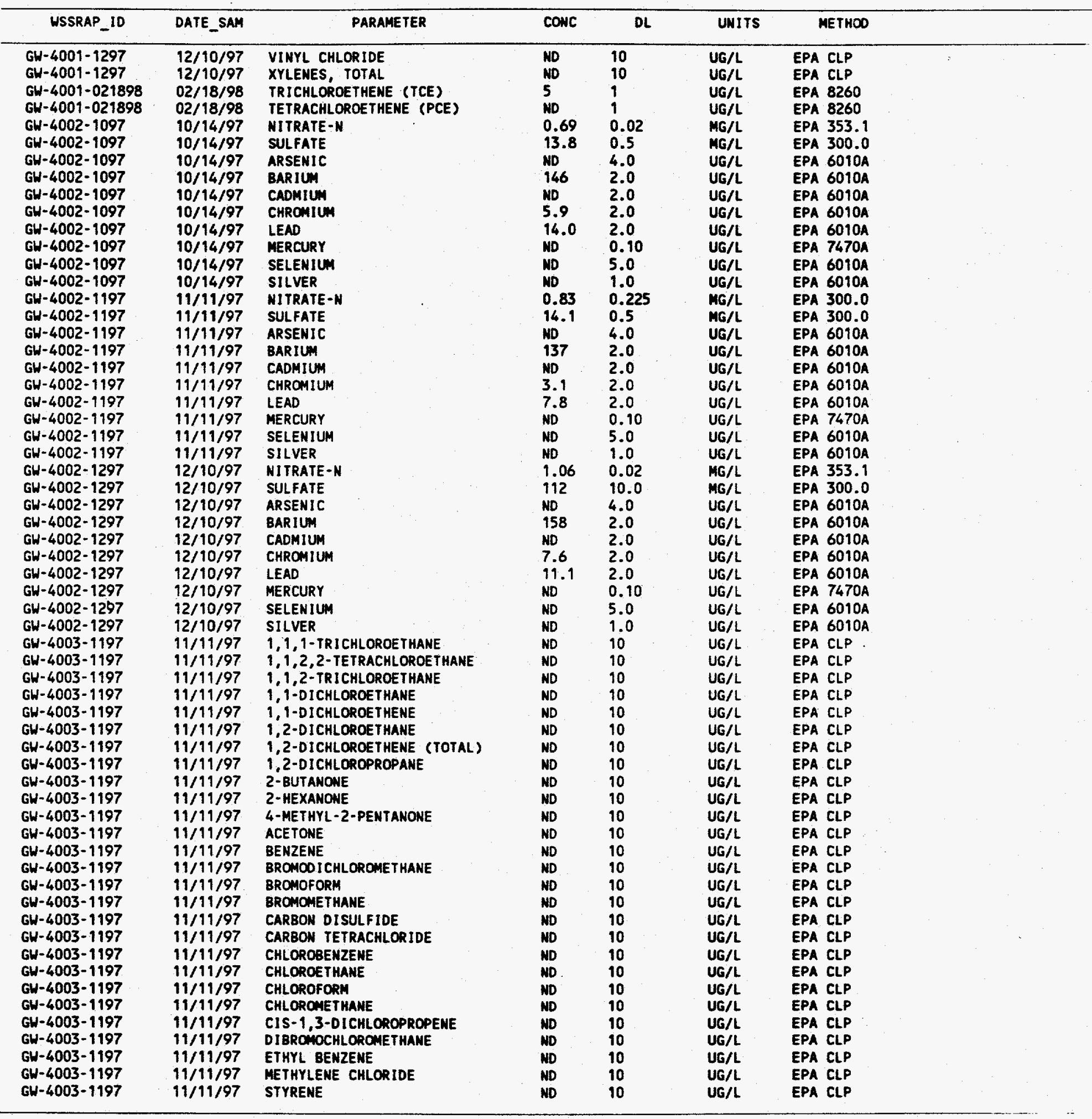




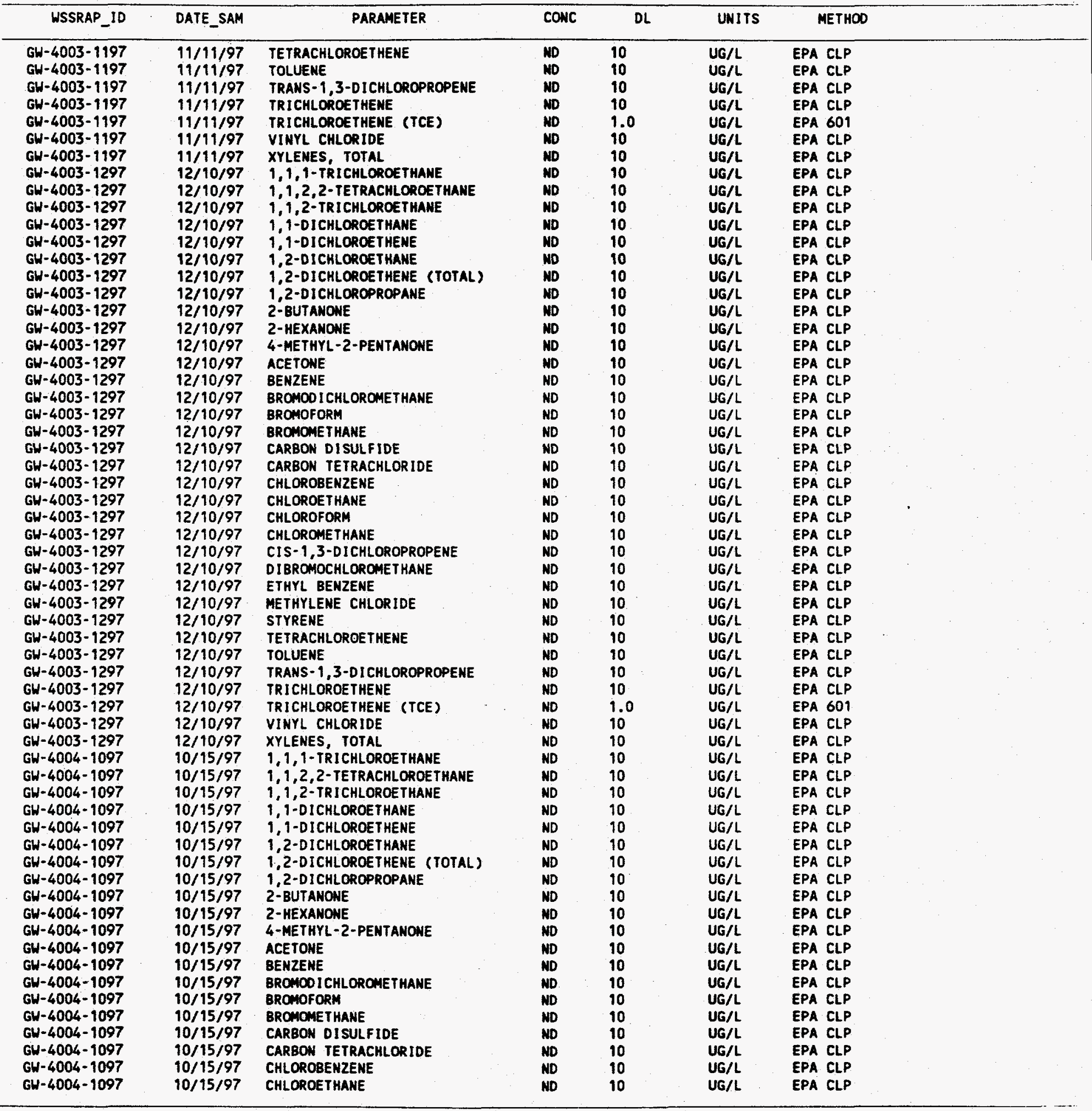




\begin{tabular}{|c|c|c|c|c|c|c|}
\hline WSSRAP_1D & DATE_SAM & PARAMETER & CONC & $\mathrm{DL}$ & UNITS & METHOD \\
\hline 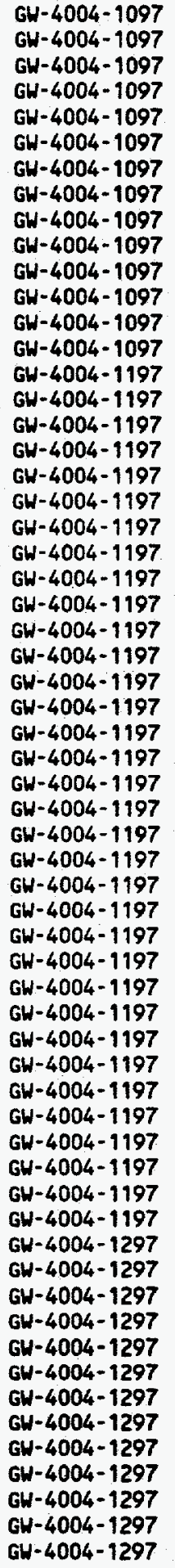 & $\begin{array}{l}10 / 15 / 97 \\
10 / 15 / 97 \\
10 / 15 / 97 \\
10 / 15 / 97 \\
10 / 15 / 97 \\
10 / 15 / 97 \\
10 / 15 / 97 \\
10 / 15 / 97 \\
10 / 15 / 97 \\
10 / 15 / 97 \\
10 / 15 / 97 \\
10 / 15 / 97 \\
10 / 15 / 97 \\
10 / 15 / 97 \\
11 / 11 / 97 \\
11 / 11 / 97 \\
11 / 11 / 97 \\
11 / 11 / 97 \\
11111 / 97 \\
111 / 11 / 97 \\
11 / 11 / 97 \\
11 / 11 / 97 \\
11 / 11 / 97 \\
11111 / 97 \\
11111 / 97 \\
11 / 11 / 97 \\
11 / 11 / 97 \\
11111 / 97 \\
11111 / 97 \\
11111 / 97 \\
11 / 11 / 97 \\
11 / 11 / 97 \\
11 / 11 / 97 \\
11111 / 97 \\
11111 / 97 \\
11 / 11 / 97 \\
11 / 111 / 97 \\
11 / 111 / 97 \\
11111 / 97 \\
111111 / 97 \\
11 / 111 / 97 \\
11 / 111 / 97 \\
11 / 111 / 97 \\
111111 / 97 \\
11 / 111 / 97 \\
11 / 111 / 97 \\
11 / 111 / 97 \\
111111 / 97 \\
12 / 111 / 97 \\
12 / 11 / 97 \\
12 / 111 / 97 \\
12 / 111 / 97 \\
12 / 111 / 97 \\
12 / 111 / 97 \\
12 / 111 / 97 \\
12 / 111 / 97 \\
12 / 111 / 97 \\
12 / 111 / 97 \\
12 / 11 / 97 \\
12 / 11 / 97 \\
12 / 111 / 97 \\
\end{array}$ & 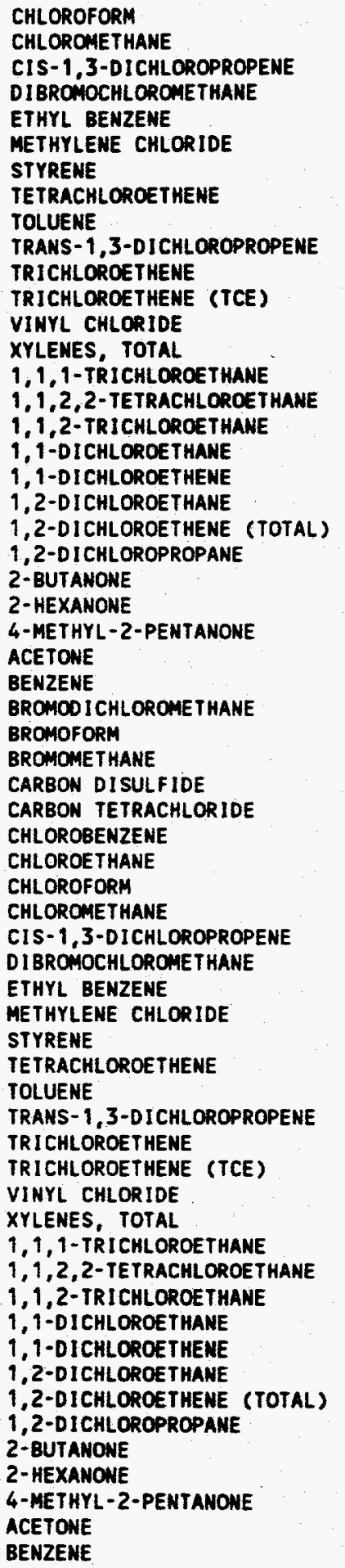 & $\begin{array}{l}\text { ND } \\
\text { ND } \\
\text { ND } \\
\text { ND } \\
\text { ND } \\
\text { ND } \\
\text { ND } \\
\text { ND } \\
\text { ND } \\
\text { ND } \\
\text { ND } \\
\text { ND } \\
\text { ND } \\
\text { ND } \\
\text { ND } \\
\text { ND } \\
\text { ND } \\
\text { ND } \\
\text { ND } \\
\text { ND } \\
\text { ND } \\
\text { ND } \\
\text { ND } \\
\text { ND } \\
\text { ND } \\
\text { ND } \\
\text { ND } \\
\text { ND } \\
\text { ND } \\
\text { ND } \\
\text { ND } \\
\text { ND } \\
\text { ND } \\
\text { ND } \\
\text { ND } \\
\text { ND } \\
\text { ND } \\
\text { ND } \\
\text { ND } \\
\text { ND } \\
\text { ND } \\
\text { ND } \\
\text { ND } \\
\text { ND } \\
\text { ND } \\
\text { ND } \\
\text { ND } \\
\text { ND } \\
\text { ND } \\
\text { ND } \\
\text { ND } \\
\text { ND } \\
\text { ND } \\
\text { ND } \\
\text { ND } \\
\text { ND } \\
\text { ND } \\
\text { ND } \\
\text { ND } \\
\text { ND } \\
\text { ND }\end{array}$ & $\begin{array}{l}10 \\
10 \\
10 \\
10 \\
10 \\
10 \\
10 \\
10 \\
10 \\
10 \\
10 \\
1.0 \\
10 \\
10 \\
10 \\
10 \\
10 \\
10 \\
10 \\
10 \\
10 \\
10 \\
10 \\
10 \\
10 \\
10 \\
10 \\
10 \\
10 \\
10 \\
10 \\
10 \\
10 \\
10 \\
10 \\
10 \\
10 \\
10 \\
10 \\
10 \\
10 \\
10 \\
10 \\
10 \\
10 \\
1.0 \\
10 \\
10 \\
10 \\
10 \\
10 \\
10 \\
10 \\
10 \\
10 \\
10 \\
10 \\
10 \\
10 \\
10 \\
10\end{array}$ & 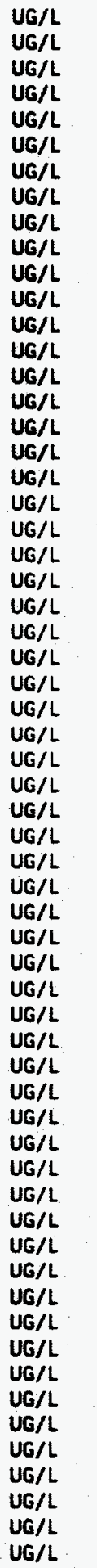 & 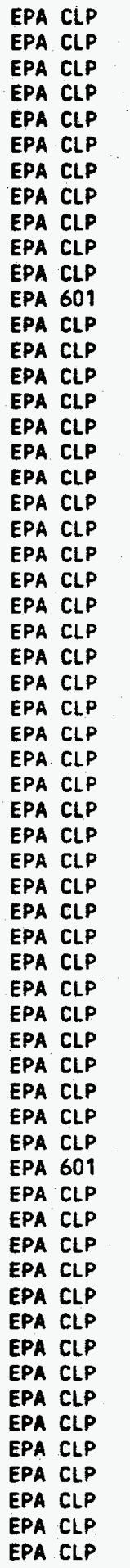 \\
\hline
\end{tabular}




\begin{tabular}{|c|c|c|c|c|c|c|}
\hline USSRAP_ID & DATE_SAM & PARAMETER & CONC & $D L$ & UNITS & METHOD \\
\hline 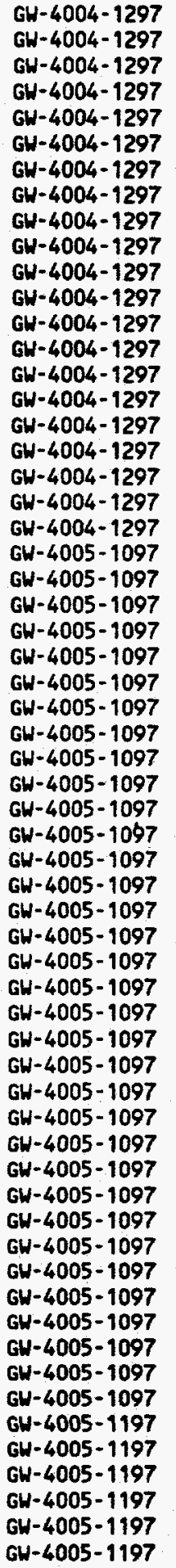 & $\begin{array}{l}12 / 11 / 97 \\
12 / 11 / 97 \\
12 / 11 / 97 \\
12 / 11 / 97 \\
12 / 11 / 97 \\
12 / 11 / 97 \\
12 / 11 / 97 \\
12 / 11 / 97 \\
12 / 11 / 97 \\
12 / 11 / 97 \\
12 / 11 / 97 \\
12 / 11 / 97 \\
12 / 11 / 97 \\
12 / 11 / 97 \\
12 / 11 / 97 \\
12 / 11 / 97 \\
12 / 11 / 97 \\
12 / 11 / 97 \\
12 / 11 / 97 \\
12 / 11 / 97 \\
12 / 11 / 97 \\
10 / 16 / 97 \\
10 / 16 / 97 \\
10 / 16 / 97 \\
10 / 16 / 97 \\
10 / 16 / 97 \\
10 / 16 / 97 \\
10 / 16 / 97 \\
10 / 16 / 97 \\
10 / 16 / 97 \\
10 / 16 / 97 \\
10 / 16 / 97 \\
10 / 16 / 97 \\
10 / 16 / 97 \\
10 / 16 / 97 \\
10 / 16 / 97 \\
10 / 16 / 97 \\
10 / 16 / 97 \\
10 / 16 / 97 \\
10 / 16 / 97 \\
10 / 16 / 97 \\
10 / 16 / 97 \\
10 / 16 / 97 \\
10 / 16 / 97 \\
10 / 16 / 97 \\
10 / 16 / 97 \\
10 / 16 / 97 \\
10 / 16 / 97 \\
10 / 16 / 97 \\
10 / 16 / 97 \\
10 / 16 / 97 \\
10 / 16 / 97 \\
10 / 16 / 97 \\
10 / 16 / 97 \\
10 / 16 / 97 \\
11 / 111 / 97 \\
11 / 111 / 97 \\
11 / 111 / 97 \\
11 / 11 / / 97 \\
11 / 11 / 97 \\
11 / 111 / 97\end{array}$ & 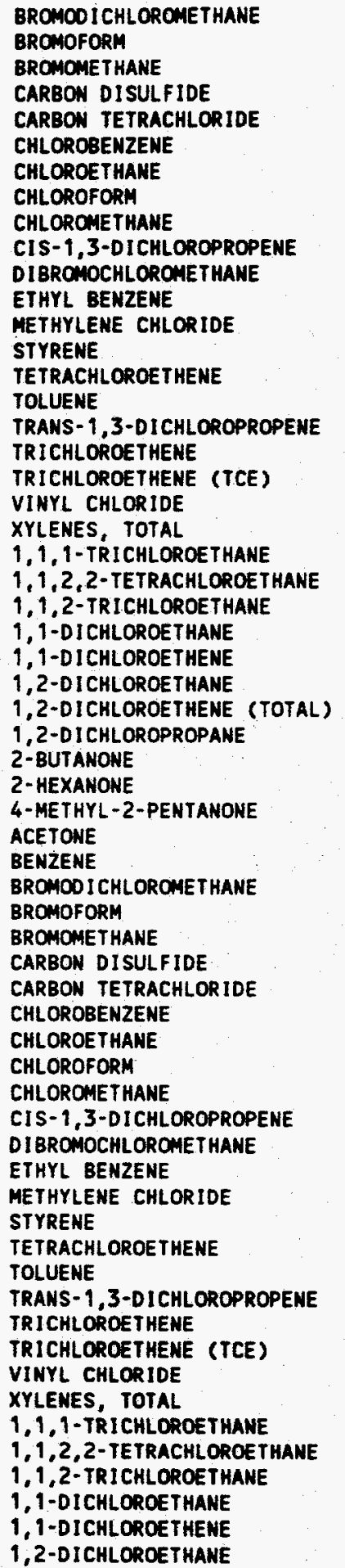 & $\begin{array}{l}\text { ND } \\
\text { ND } \\
\text { ND } \\
\text { ND } \\
\text { ND } \\
\text { ND } \\
\text { ND } \\
\text { ND } \\
\text { ND } \\
\text { ND } \\
\text { ND } \\
\text { ND } \\
\text { ND } \\
\text { ND } \\
\text { ND } \\
\text { ND } \\
\text { ND } \\
\text { ND } \\
\text { ND } \\
\text { ND } \\
\text { ND } \\
\text { ND } \\
\text { ND } \\
\text { ND } \\
\text { ND } \\
\text { ND } \\
\text { ND } \\
\text { ND } \\
\text { ND } \\
\text { ND } \\
\text { ND } \\
\text { ND } \\
\text { ND } \\
\text { ND } \\
\text { ND } \\
\text { ND } \\
\text { ND } \\
\text { ND } \\
\text { ND } \\
\text { ND } \\
\text { ND } \\
\text { ND } \\
\text { ND } \\
\text { ND } \\
\text { ND } \\
\text { ND } \\
\text { ND } \\
\text { ND } \\
\text { ND } \\
\text { ND } \\
\text { ND } \\
\text { ND } \\
\text { ND } \\
\text { ND } \\
\text { ND } \\
\text { ND } \\
\text { ND } \\
\text { ND } \\
\text { ND } \\
\text { ND } \\
\text { ND }\end{array}$ & $\begin{array}{l}10 \\
10 \\
10 \\
10 \\
10 \\
10 \\
10 \\
10 \\
10 \\
10 \\
10 \\
10 \\
10 \\
10 \\
10 \\
10 \\
10 \\
10 \\
1.0 \\
10 \\
10 \\
10 \\
10 \\
10 \\
10 \\
10 \\
10 \\
10 \\
10 \\
10 \\
10 \\
10 \\
10 \\
10 \\
10 \\
10 \\
10 \\
10 \\
10 \\
10 \\
10 \\
10 \\
10 \\
10 \\
10 \\
10 \\
10 \\
10 \\
10 \\
10 \\
10 \\
10 \\
1.0 \\
10 \\
10 \\
10 \\
10 \\
10 \\
10 \\
10 \\
10\end{array}$ & 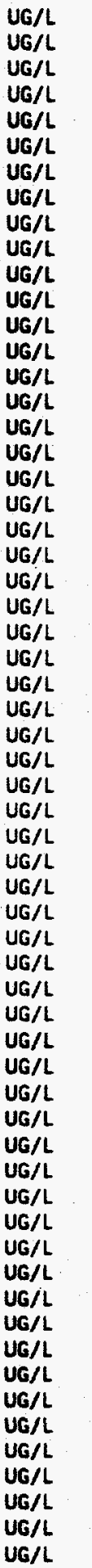 & 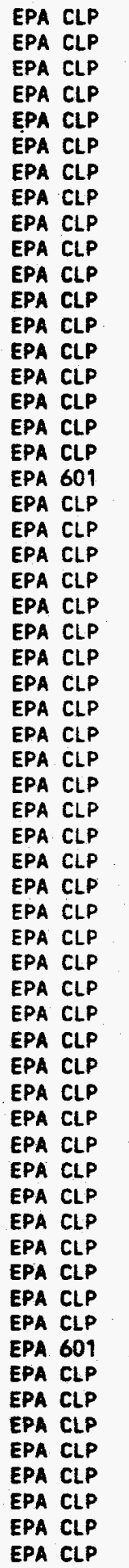 \\
\hline
\end{tabular}




\begin{tabular}{|c|c|c|c|c|c|c|c|}
\hline WSSRAP_ID & DATE_SAM & PARAMETER & CONC & DL & UNITS & METHOD & \\
\hline $\begin{array}{l}G W-4005-1197 \\
G W-4005-1197 \\
G W-4005-1197 \\
G W-4005-1197 \\
G W-4005-1197 \\
G W-4005-1197 \\
G W-4005-1197 \\
G W-4005-1197 \\
G W-4005-1197 \\
G W-4005-1197 \\
G W-4005-1197 \\
G W-4005-1197 \\
G W-4005-1197 \\
G W-4005-1197 \\
G W-4005-1197 \\
G W-4005-1197 \\
G W-4005-1197 \\
G W-4005-1197 \\
G W-4005-1197 \\
G W-4005-1197 \\
G W-4005-1197 \\
G W-4005-1197 \\
G W-4005-1197 \\
G W-4005-1197 \\
G W-4005-1197 \\
G W-4005-1197 \\
G W-4005-1197 \\
G W-4005-1197 \\
G W-4005-1197 \\
G W-4005-1297 \\
G W-4005-1297 \\
G W-4005-1297 \\
G W-4005-1297 \\
G W-4005-1297 \\
G W-4005-1297 \\
G W-4005-1297 \\
G W-4005-1297 \\
G W-4005-1297 \\
G W-4005-1297 \\
G W-4005-1297 \\
G W-4005-1297 \\
G W-4005-1297 \\
G W-4005-1297 \\
G W-4005-1297 \\
G W-4005-1297 \\
G W-4005-1297 \\
G W-4005-1297 \\
G W-4005-1297 \\
G W-4005-1297 \\
G W-4005-1297 \\
G W-4005-1297 \\
G W-4005-1297 \\
G W-4005-1297 \\
G W-4005-1297 \\
G W-4005-1297 \\
G W-4005-1297 \\
G W-4005-1297 \\
G W-4005-1297 \\
G W-4005-1297 \\
G W-4005-1297\end{array}$ & $\begin{array}{l}11 / 11 / 97 \\
11 / 11 / 97 \\
11 / 11 / 97 \\
11 / 11 / 97 \\
11 / 11 / 97 \\
11 / 11 / 97 \\
11 / 11 / 97 \\
11 / 11 / 97 \\
11 / 11 / 97 \\
11 / 11 / 97 \\
11 / 11 / 97 \\
11 / 11 / 97 \\
11 / 11 / 97 \\
11 / 11 / 97 \\
11 / 11 / 97 \\
11 / 11 / 97 \\
11 / 11 / 97 \\
11 / 11 / 97 \\
11 / 11 / 97 \\
11 / 11 / 97 \\
11 / 11 / 97 \\
11 / 11 / 97 \\
11 / 11 / 97 \\
11 / 11 / 97 \\
11 / 11 / 97 \\
11 / 11 / 97 \\
11 / 11 / 97 \\
11 / 11 / 97 \\
11 / 11 / 97 \\
12 / 10 / 97 \\
12 / 11 / 97 \\
12 / 11 / 97 \\
12 / 11 / 97 \\
12 / 11 / 97 \\
12 / 11 / 97 \\
12 / 11 / 97 \\
12 / 11 / 97 \\
12 / 11 / 97 \\
12 / 11 / 97 \\
12 / 11 / 97 \\
12 / 11 / 97 \\
12 / 11 / 97 \\
12 / 11 / 97 \\
12 / 11 / 97 \\
12 / 11 / 97 \\
12 / 11 / 97 \\
12 / 11 / 97 \\
12 / 11 / 97 \\
12 / 11 / 97 \\
12 / 11 / 97 \\
12 / 11 / 97 \\
12 / 11 / 97 \\
12 / 11 / 97 \\
12 / 11 / 97 \\
12 / 11 / 97 \\
12 / 11 / 97 \\
12 / 11 / 97 \\
12 / 11 / 97 \\
12 / 11 / 97 \\
12 / 11 / 97 \\
12 / 11 / 97\end{array}$ & 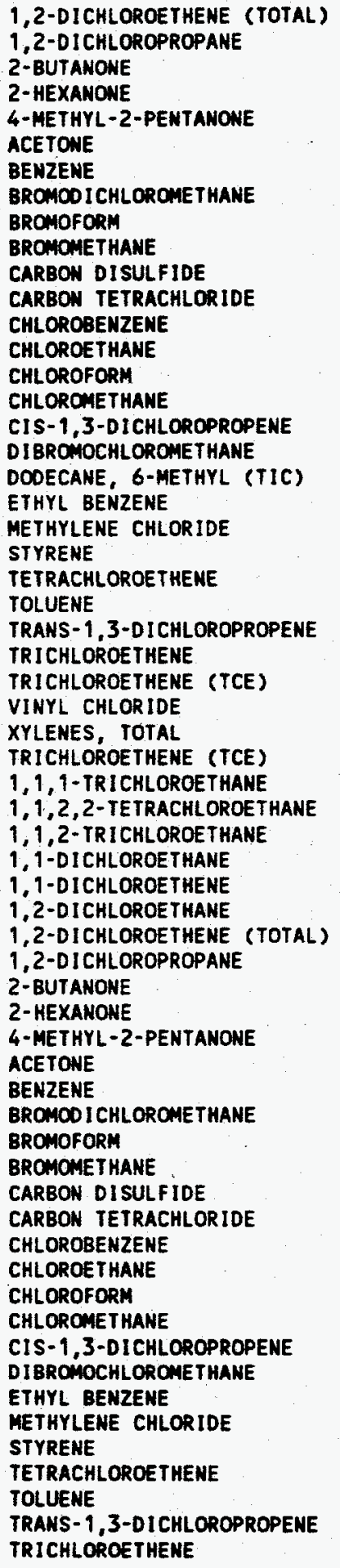 & $\begin{array}{l}\text { ND } \\
\text { ND } \\
\text { ND } \\
\text { ND } \\
\text { ND } \\
\text { ND } \\
\text { ND } \\
\text { ND } \\
\text { ND } \\
\text { ND } \\
\text { ND } \\
\text { ND } \\
\text { ND } \\
\text { ND } \\
\text { ND } \\
\text { ND } \\
\text { ND } \\
6 \\
\text { ND } \\
\text { ND } \\
\text { ND } \\
\text { ND } \\
\text { ND } \\
\text { ND } \\
\text { ND } \\
\text { ND } \\
\text { ND } \\
\text { ND } \\
\text { ND } \\
\text { ND } \\
\text { ND } \\
\text { ND } \\
\text { ND } \\
\text { ND } \\
\text { ND } \\
\text { ND } \\
\text { ND } \\
\text { ND } \\
\text { ND } \\
\text { ND } \\
\text { ND } \\
\text { ND } \\
\text { ND } \\
\text { ND } \\
\text { ND } \\
\text { ND } \\
\text { ND } \\
\text { ND } \\
\text { ND } \\
\text { ND } \\
\text { ND } \\
\text { ND } \\
\text { ND } \\
\text { ND } \\
\text { ND } \\
\text { ND } \\
\text { ND } \\
\text { ND } \\
\text { ND } \\
\text { ND }\end{array}$ & $\begin{array}{l}10 \\
10 \\
10 \\
10 \\
10 \\
10 \\
10 \\
10 \\
10 \\
10 \\
10 \\
10 \\
10 \\
10 \\
10 \\
10 \\
10 \\
10 \\
10 \\
10 \\
10 \\
10 \\
10 \\
10 \\
10 \\
10 \\
1.0 \\
10 \\
10 \\
1.0 \\
10 \\
10 \\
10 \\
10 \\
10 \\
10 \\
10 \\
10 \\
10 \\
10 \\
10 \\
10 \\
10 \\
10 \\
10 \\
10 \\
10 \\
10 \\
10 \\
10 \\
10 \\
10 \\
10 \\
10 \\
10 \\
10 \\
10 \\
10 \\
10 \\
10 \\
10\end{array}$ & $\begin{array}{l}U G / L \\
U G / L \\
U G / L \\
U G / L \\
U G / L \\
U G / L \\
U G / L \\
U G / L \\
U G / L \\
U G / L \\
U G / L \\
U G / L \\
U G / L \\
U G / L \\
U G / L \\
U G / L \\
U G / L \\
U G / L \\
U G / L \\
U G / L \\
U G / L \\
U G / L \\
U G / L \\
U G / L \\
U G / L \\
U G / L \\
U G / L \\
U G / L \\
U G / L \\
U G / L \\
U G / L \\
U G / L \\
U G / L \\
U G / L \\
U G / L \\
U G / L \\
U G / L \\
U G / L \\
U G / L \\
U G / L \\
U G / L \\
U G / L \\
U G / L \\
U G / L \\
U G / L \\
U G / L \\
U G / L \\
U G / L \\
U G / L \\
U G / L \\
U G / L \\
U G / L \\
U G / L \\
U G / L \\
U G / L \\
U G / L \\
U G / L \\
U G / L \\
U G / L \\
U\end{array}$ & $\begin{array}{l}\text { EPA CLP } \\
\text { EPA CLP } \\
\text { EPA CLP } \\
\text { EPA CLP } \\
\text { EPA CLP } \\
\text { EPA CLP } \\
\text { EPA CLP } \\
\text { EPA CLP } \\
\text { EPA CLP } \\
\text { EPA CLP } \\
\text { EPA CLP } \\
\text { EPA CLP } \\
\text { EPA CLP } \\
\text { EPA CLP } \\
\text { EPA CLP } \\
\text { EPA CLL } \\
\text { EPA CLP } \\
\text { EPA CLP } \\
\text { EPA CLP } \\
\text { EPA CLP } \\
\text { EPA CLP } \\
\text { EPA CLP } \\
\text { EPA CLP } \\
\text { EPA CLP } \\
\text { EPA CLL } \\
\text { EPA CLP } \\
\text { EPA } 601 \\
\text { EPA CLP } \\
\text { EPA CLP } \\
\text { EPA } 601 \\
\text { EPA CLP } \\
\text { EPA CLP } \\
\text { EPA CLP } \\
\text { EPA CLP } \\
\text { EPA CLP } \\
\text { EPA CLP } \\
\text { EPA CLP } \\
\text { EPA CLP } \\
\text { EPA CLP } \\
\text { EPA CLP } \\
\text { EPA CLP } \\
\text { EPA CLP } \\
\text { EPA CLP } \\
\text { EPA CLP } \\
\text { EPA CLP } \\
\text { EPA CLP } \\
\text { EPA CLP } \\
\text { EPA CLP } \\
\text { EPA CLP } \\
\text { EPA CLP } \\
\text { EPA CLP } \\
\text { EPA CLP } \\
\text { EPA CLP } \\
\text { EPA CLP } \\
\text { EPA CLP } \\
\text { EPA CLP } \\
\text { EPA CLP } \\
\text { EPA CLP } \\
\text { EPA CLP } \\
\text { EPA CLP } \\
\text { EPA CLP }\end{array}$ & \\
\hline
\end{tabular}




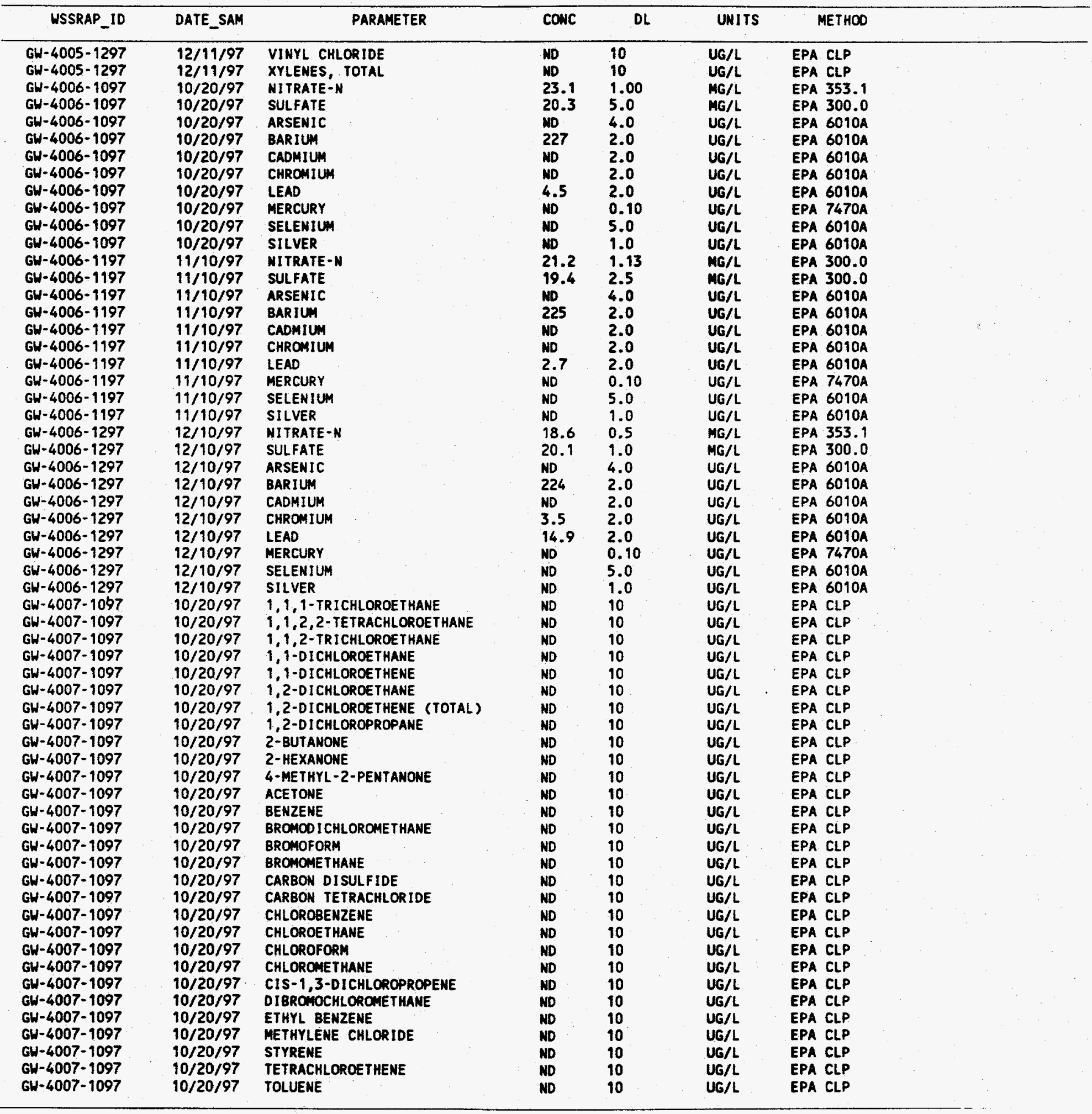




\begin{tabular}{|c|c|c|c|c|c|c|}
\hline WSSRAP_ID & DATE_SAM & PARAMETER & CONC & $D L$ & UNLTS & METHOD \\
\hline $\begin{array}{l}\text { GW-4007-1097 } \\
G W-4007-1097 \\
G W-4007-1097 \\
G W-4007-1097 \\
G W-4007-1097 \\
G W-4007-1197 \\
G W-4007-1197 \\
G W-4007-1197 \\
G W-4007-1197 \\
G W-4007-1197 \\
G W-4007-1197 \\
G W-4007-1197 \\
G W-4007-1197 \\
G W-4007-1197 \\
G W-4007-1197 \\
G W-4007-1197 \\
G W-4007-1197 \\
G W-4007-1197 \\
G W-4007-1197 \\
G W-4007-1197 \\
G W-4007-1197 \\
G W-4007-1197 \\
G W-4007-1197 \\
G W-4007-1197 \\
G W-4007-1197 \\
G W-4007-1197 \\
G W-4007-1197 \\
G W-4007-1197 \\
G W-4007-1197 \\
G W-4007-1197 \\
G W-4007-1197 \\
G W-4007-1197 \\
G W-4007-1197 \\
G W-4007-1197 \\
G W-4007-1197 \\
G W-4007-1197 \\
G W-4007-1197 \\
G W-4007-1197 \\
G W-4007-1197 \\
G W-4007-1297 \\
G W-4007-1297 \\
G W-4007-1297 \\
G W-4007-1297 \\
G W-4007-1297 \\
G W-4007-1297 \\
G W-4007-1297 \\
G W-4007-1297 \\
G W-4007-1297 \\
G W-4007-1297 \\
G W-4007-1297 \\
G W-4007-1297 \\
G W-4007-1297 \\
G W-4007-1297 \\
G W-4007-1297 \\
G W-4007-1297 \\
G W-4007-1297 \\
G-4007-1297 \\
\text { GW }\end{array}$ & $\begin{array}{l}10 / 20 / 97 \\
10 / 20 / 97 \\
10 / 20 / 97 \\
10 / 20 / 97 \\
10 / 20 / 97 \\
11 / 10 / 97 \\
11 / 10 / 97 \\
11 / 10 / 97 \\
11 / 10 / 97 \\
11 / 10 / 97 \\
11 / 10 / 97 \\
11 / 10 / 97 \\
11 / 10 / 97 \\
11 / 10 / 97 \\
11 / 10 / 97 \\
11 / 10 / 97 \\
11 / 10 / 97 \\
11 / 10 / 97 \\
11 / 10 / 97 \\
11 / 10 / 97 \\
11 / 10 / 97 \\
11 / 10 / 97 \\
11 / 10 / 97 \\
11 / 10 / 97 \\
11 / 10 / 97 \\
11 / 10 / 97 \\
11 / 10 / 97 \\
11 / 10 / 97 \\
11110 / 97 \\
11 / 10 / 97 \\
11 / 10 / 97 \\
11 / 10 / 97 \\
11 / 10 / 97 \\
11 / 10 / 97 \\
111 / 10 / 97 \\
11 / 10 / 97 \\
11 / 10 / 97 \\
11 / 10 / 97 \\
11 / 10 / 97 \\
12 / 10 / 97 \\
12 / 10 / 97 \\
12 / 10 / 97 \\
12 / 10 / 97 \\
12 / 10 / 97 \\
12 / 10 / 97 \\
12 / 10 / 97 \\
12 / 10 / 97 \\
12 / 10 / 97 \\
12 / 10 / 97 \\
12 / 10 / 97 \\
12 / 10 / 97 \\
12 / 10 / 97 \\
12 / 10 / 97 \\
12 / 10 / 97 \\
12 / 10 / 97 \\
12 / 10 / 97 \\
12 / 10 / 97 \\
12 / 10 / 97 \\
12 / 10 / 97 \\
12 / 10 / 97 \\
12 / 10 / 97\end{array}$ & 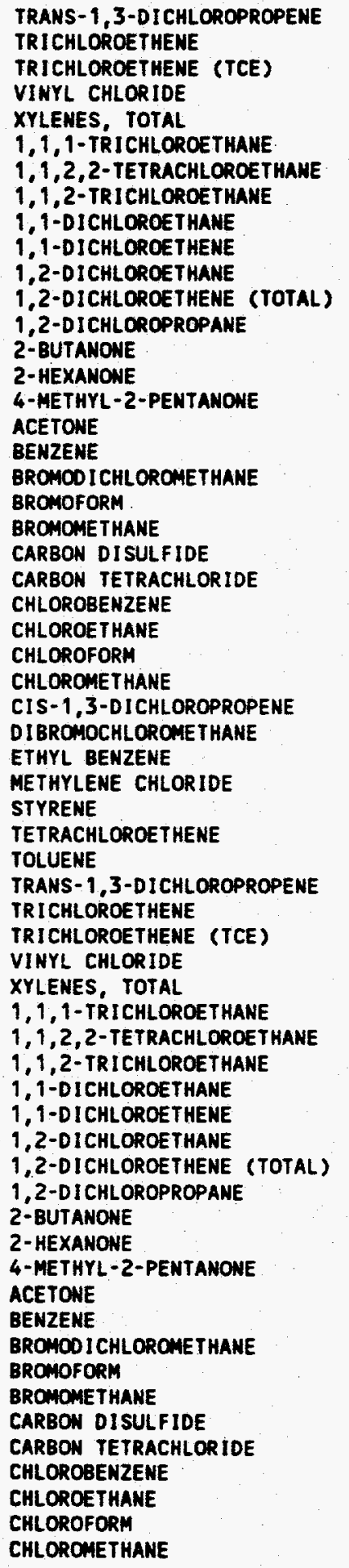 & $\begin{array}{l}\text { ND } \\
\text { ND } \\
\text { ND } \\
\text { ND } \\
\text { ND } \\
\text { ND } \\
\text { ND } \\
\text { ND } \\
\text { ND } \\
\text { ND } \\
\text { ND } \\
\text { ND } \\
\text { ND } \\
\text { ND } \\
\text { ND } \\
\text { ND } \\
\text { ND } \\
\text { ND } \\
\text { ND } \\
\text { ND } \\
\text { ND } \\
\text { ND } \\
\text { ND } \\
\text { ND } \\
\text { ND } \\
\text { ND } \\
\text { ND } \\
\text { ND } \\
\text { ND } \\
\text { ND } \\
\text { ND } \\
\text { ND } \\
\text { ND } \\
\text { ND } \\
\text { ND } \\
\text { ND } \\
\text { ND } \\
\text { ND } \\
\text { ND } \\
\text { ND } \\
\text { ND } \\
\text { ND } \\
\text { ND } \\
\text { ND } \\
\text { ND } \\
\text { ND } \\
\text { ND } \\
\text { ND } \\
\text { ND } \\
\text { ND } \\
\text { ND } \\
\text { ND } \\
\text { ND } \\
\text { ND } \\
\text { ND } \\
\text { ND } \\
\text { ND } \\
\text { ND } \\
\text { ND } \\
\text { ND } \\
\text { ND }\end{array}$ & $\begin{array}{l}10 \\
10 \\
1.0 \\
10 \\
10 \\
10 \\
10 \\
10 \\
10 \\
10 \\
10 \\
10 \\
10 \\
10 \\
10 \\
10 \\
10 \\
10 \\
10 \\
10 \\
10 \\
10 \\
10 \\
10 \\
10 \\
10 \\
10 \\
10 \\
10 \\
10 \\
10 \\
10 \\
10 \\
10 \\
10 \\
10 \\
1.0 \\
10 \\
10 \\
10 \\
10 \\
10 \\
10 \\
10 \\
10 \\
10 \\
10 \\
10 \\
10 \\
10 \\
10 \\
10 \\
10 \\
10 \\
10 \\
10 \\
10 \\
10 \\
10 \\
10 \\
10 \\
\end{array}$ & 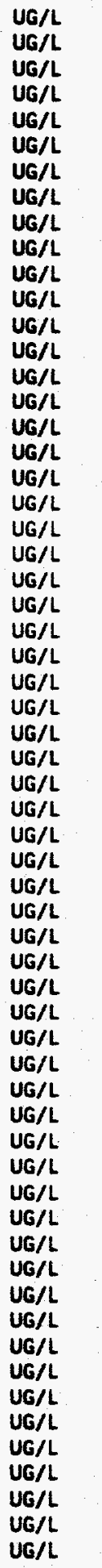 & 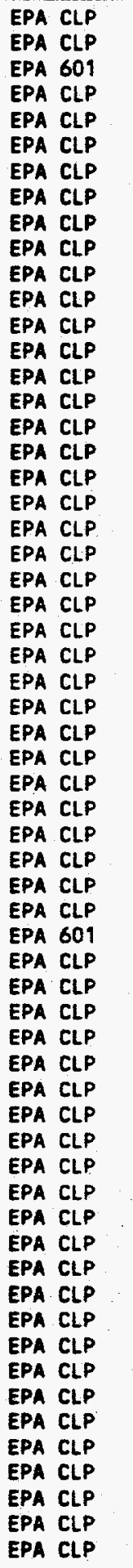 \\
\hline
\end{tabular}




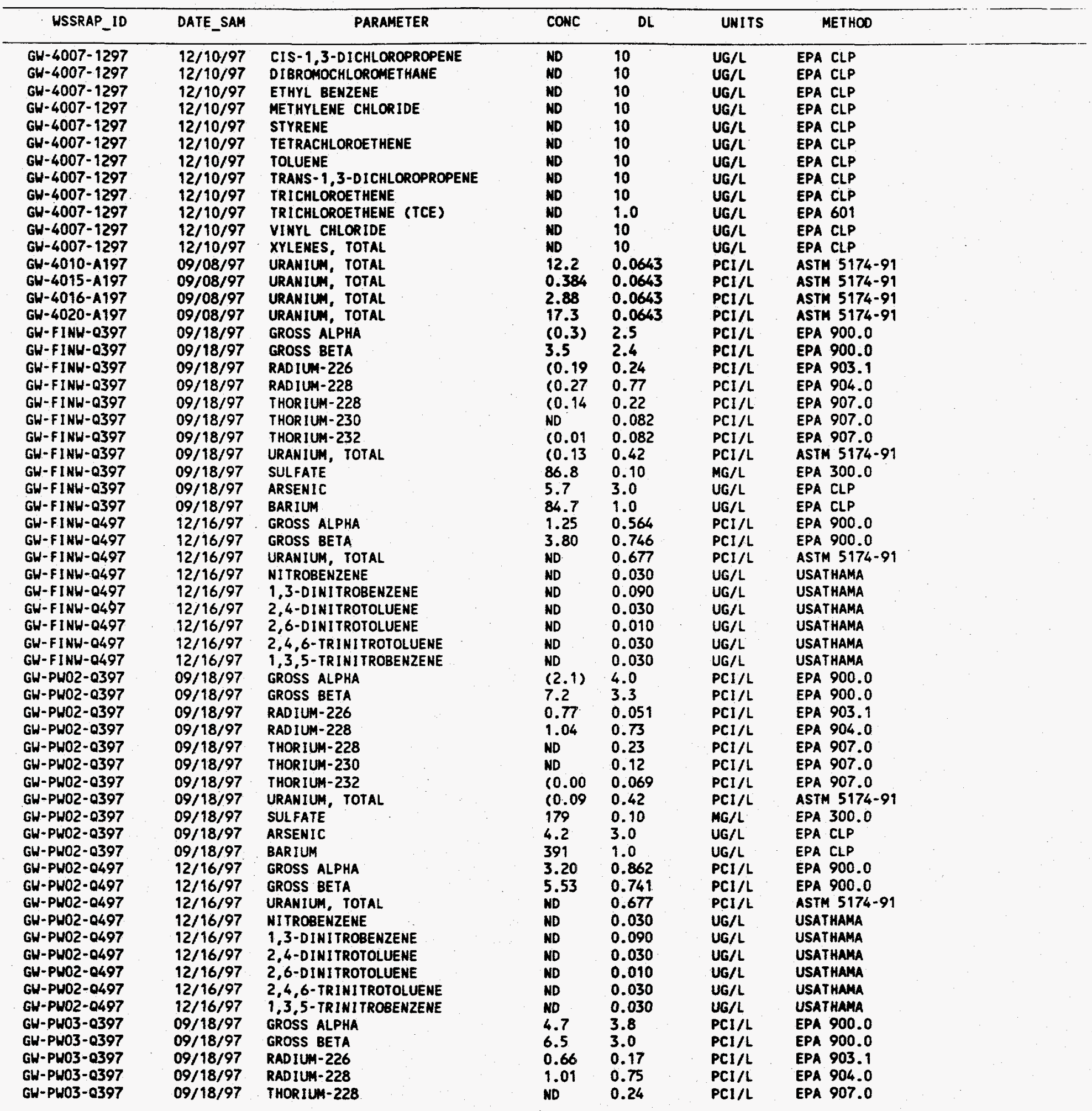




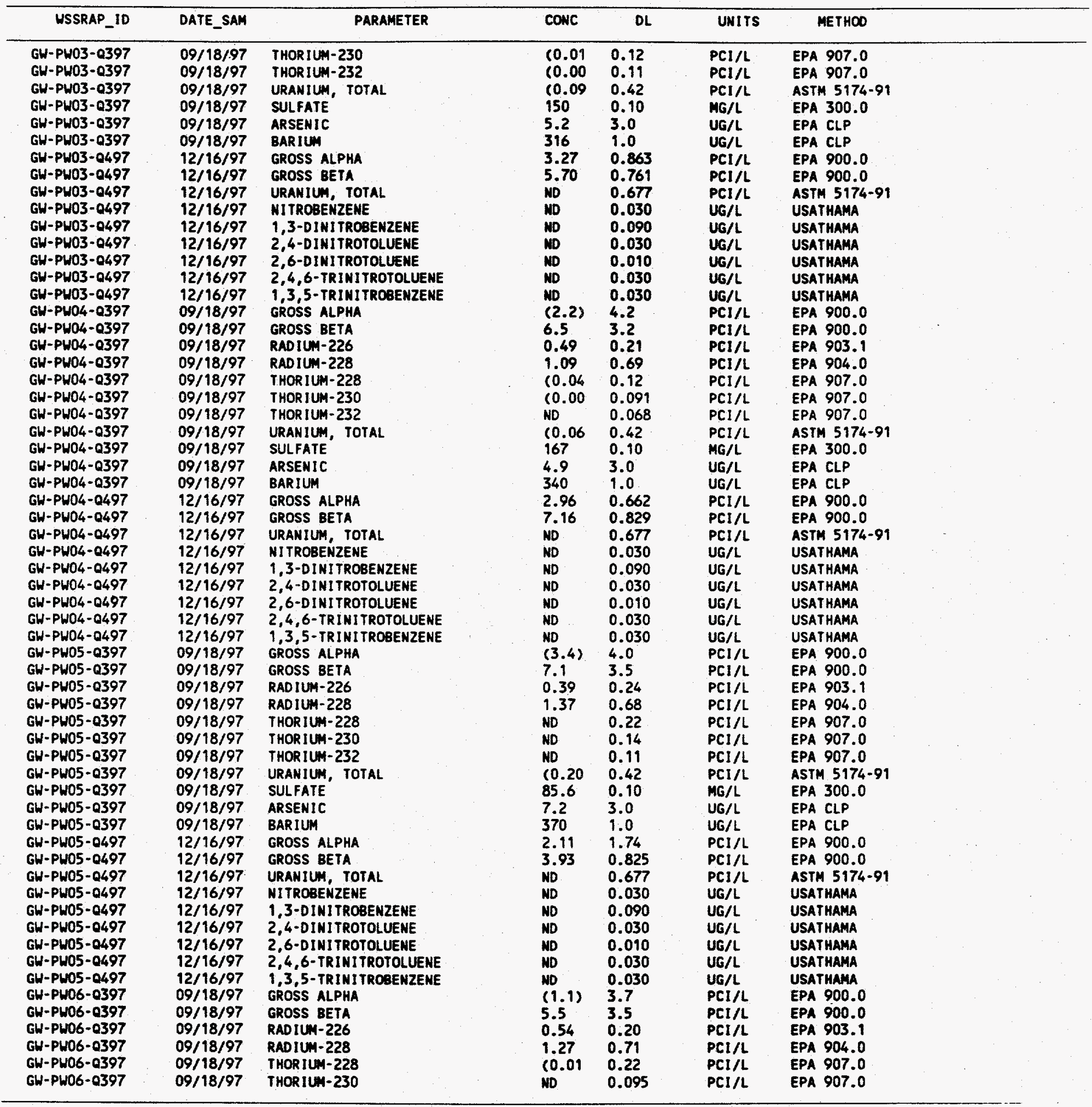




\begin{tabular}{|c|c|c|c|c|c|c|}
\hline WSSRAP_ID & DATE_SAM & PARAMETER & Conc & $D L$ & UNITS & METHOD \\
\hline 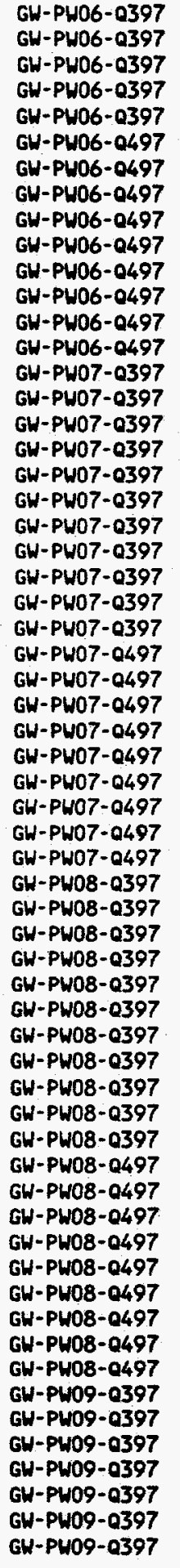 & $\begin{array}{l}09 / 18 / 97 \\
09 / 18 / 97 \\
09 / 18 / 97 \\
09 / 18 / 97 \\
09 / 18 / 97 \\
12 / 16 / 97 \\
12 / 16 / 97 \\
12 / 16 / 97 \\
12 / 16 / 97 \\
12 / 16 / 97 \\
12 / 16 / 97 \\
12 / 16 / 97 \\
12 / 16 / 97 \\
12 / 16 / 97 \\
09 / 18 / 97 \\
09 / 18 / 97 \\
09 / 18 / 97 \\
09 / 18 / 97 \\
09 / 18 / 97 \\
09 / 18 / 97 \\
09 / 18 / 97 \\
09 / 18 / 97 \\
09 / 18 / 97 \\
09 / 18 / 97 \\
09 / 18 / 97 \\
12 / 16 / 97 \\
12 / 16 / 97 \\
12 / 16 / 97 \\
12 / 16 / 97 \\
12 / 16 / 97 \\
12 / 16 / 97 \\
12 / 16 / 97 \\
12 / 16 / 97 \\
12 / 16 / 97 \\
09 / 18 / 97 \\
09 / 18 / 97 \\
09 / 18 / 97 \\
09 / 18 / 97 \\
09 / 18 / 97 \\
09 / 18 / 97 \\
09 / 18 / 97 \\
09 / 18 / 97 \\
09 / 18 / 97 \\
09 / 18 / 97 \\
09 / 18 / 97 \\
12 / 16 / 97 \\
12 / 16 / 97 \\
12 / 16 / 97 \\
12 / 16 / 97 \\
12 / 16 / 97 \\
12 / 16 / 97 \\
12 / 16 / 97 \\
12 / 16 / 97 \\
12 / 16 / 97 \\
09 / 18 / 97 \\
09 / 18 / 97 \\
09 / 18 / 97 \\
09 / 18 / 97 \\
09 / 18 / 97 \\
09 / 18 / 97 \\
09 / 18 / 97\end{array}$ & 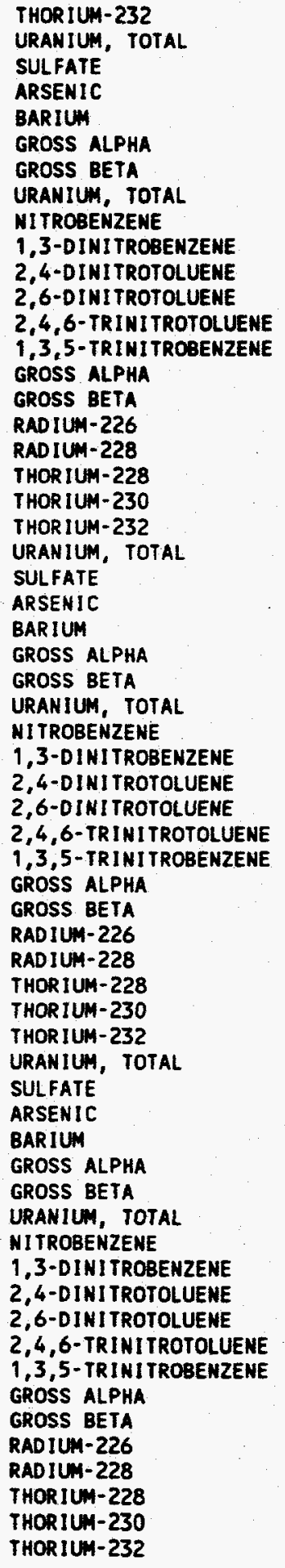 & $\begin{array}{l}\text { ND } \\
\text { (0.19 } \\
118 \\
6.3 \\
326 \\
2.80 \\
4.31 \\
\text { ND } \\
\text { ND } \\
\text { ND } \\
\text { ND } \\
\text { ND } \\
\text { ND } \\
\text { ND } \\
(2.0) \\
4.5 \\
0.68 \\
0.95 \\
(0.01 \\
\text { ND } \\
\text { ND } \\
(0.10 \\
82.6 \\
5.3 \\
433 \\
1.82 \\
4.06 \\
\text { ND } \\
\text { ND } \\
\text { ND } \\
\text { ND } \\
\text { ND } \\
\text { ND } \\
\text { ND } \\
(1.6) \\
(3.2) \\
0.59 \\
0.92 \\
\text { ND } \\
\text { ND } \\
\text { ND } \\
(0.25 \\
45.1 \\
7.6 \\
456 \\
2.93 \\
4.83 \\
\text { ND } \\
\text { ND } \\
\text { ND } \\
\text { ND } \\
\text { ND } \\
\text { ND } \\
\text { ND } \\
(1.6) \\
6.8 \\
0.93 \\
1.85 \\
\text { ND } \\
\text { ND } \\
\text { ND }\end{array}$ & $\begin{array}{l}0.076 \\
0.42 \\
0.10 \\
3.0 \\
1.0 \\
0.841 \\
0.861 \\
0.677 \\
0.030 \\
0.090 \\
0.030 \\
0.010 \\
0.030 \\
0.030 \\
3.8 \\
4.1 \\
0.26 \\
0.69 \\
0.22 \\
0.11 \\
0.11 \\
0.42 \\
0.10 \\
3.0 \\
1.0 \\
0.787 \\
0.791 \\
0.677 \\
0.030 \\
0.090 \\
0.030 \\
0.010 \\
0.030 \\
0.030 \\
5.0 \\
5.5 \\
0.20 \\
0.71 \\
0.23 \\
0.092 \\
0.050 \\
0.42 \\
0.010 \\
0.030 \\
0.030 \\
0.030 \\
0.030 \\
0.07 \\
0.07 \\
0.87 \\
0.24 \\
0.72 \\
0.11 \\
0.095\end{array}$ & 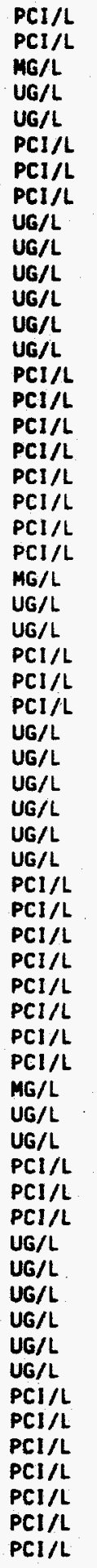 & $\begin{array}{l}\text { EPA } 907.0 \\
\text { ASTM } 5174-91 \\
\text { EPA } 300.0 \\
\text { EPA CLP } \\
\text { EPA CLP } \\
\text { EPA } 900.0 \\
\text { EPA } 900.0 \\
\text { ASTM } 5174-91 \\
\text { USATHAMA } \\
\text { USATHAMA } \\
\text { USATHAMA } \\
\text { USATHAMA } \\
\text { USATHAMA } \\
\text { USATHAMA } \\
\text { EPA } 900.0 \\
\text { EPA } 900.0 \\
\text { EPA } 903.1 \\
\text { EPA } 904.0 \\
\text { EPA } 907.0 \\
\text { EPA } 907.0 \\
\text { EPA } 907.0 \\
\text { ASTM } 5174-91 \\
\text { EPA } 300.0 \\
\text { EPA CLP } \\
\text { EPA CLP } \\
\text { EPA } 900.0 \\
\text { EPA } 900.0 \\
\text { ASTM } 5174-91 \\
\text { USATHAMA } \\
\text { USATHAMA } \\
\text { USATHAMA } \\
\text { USATHAMA } \\
\text { USATHAMA } \\
\text { USATHAMA } \\
\text { EPA 900.0 } \\
\text { EPA 900.0 } \\
\text { EPA } 903.1 \\
\text { EPA } 904.0 \\
\text { EPA } 907.0 \\
\text { EPA } 907.0 \\
\text { EPA } 907.0 \\
\text { ASTM } 5174-91 \\
\text { EPA } 300.0 \\
\text { EPA CLP } \\
\text { EPA CLP } \\
\text { EPA } 900.0 \\
\text { EPA } 900.0 \\
\text { ASTM } 5174-91 \\
\text { USATHAMA } \\
\text { USATHAMA } \\
\text { USATHAMA } \\
\text { USATHAMA } \\
\text { USATHAMA } \\
\text { USATHAMA } \\
\text { EPA } 900.0 \\
\text { EPA } 900.0 \\
\text { EPA } 903.1 \\
\text { EPA } 904.0 \\
\text { EPA } 907.0 \\
\text { EPA } 907.0 \\
\text { EPA } 907.0 \\
\end{array}$ \\
\hline
\end{tabular}




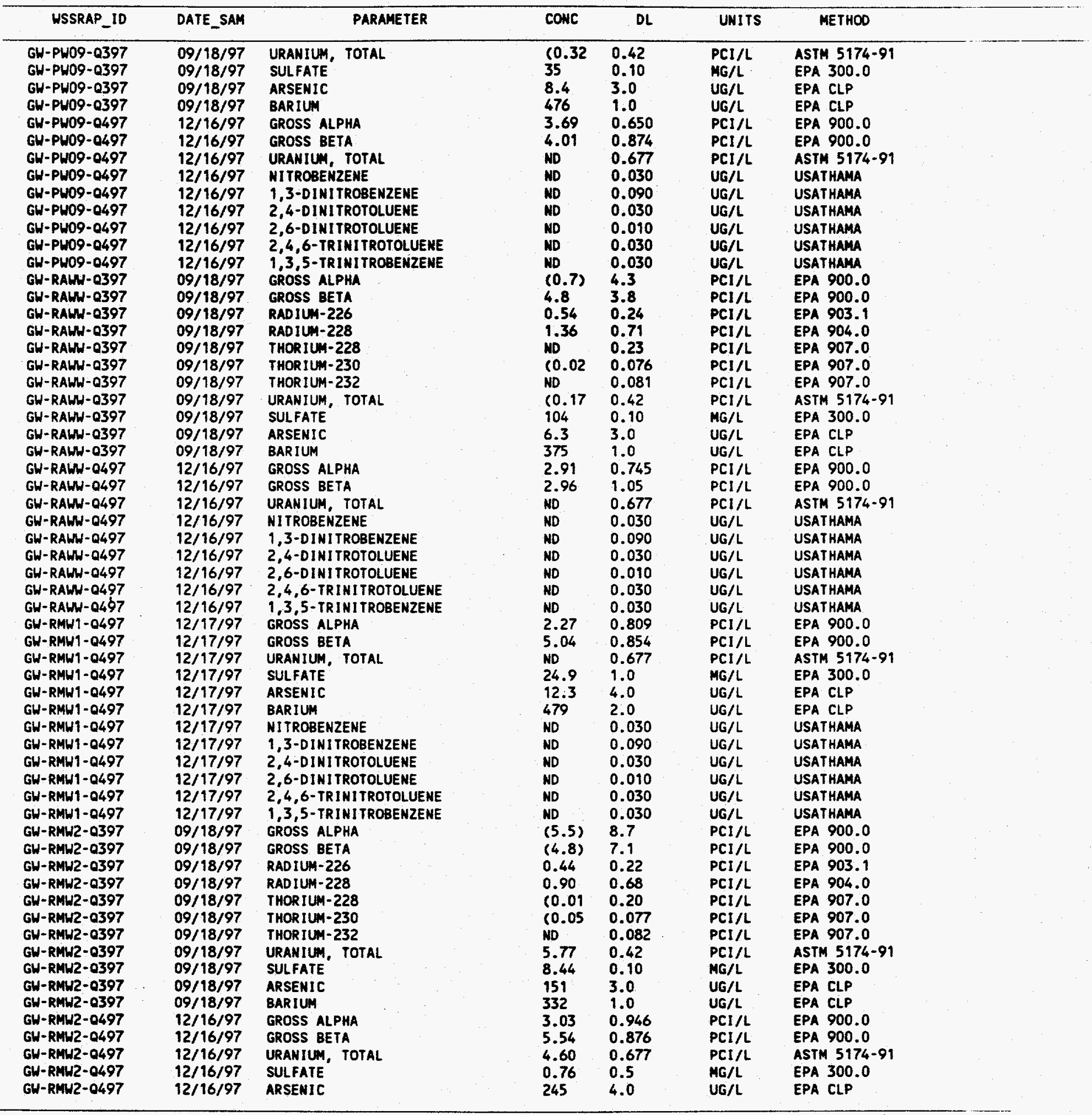




\begin{tabular}{|c|c|c|c|c|c|c|}
\hline USSRAP_ID & DATE_SAM & PARAMETER & CONC & DL & UNITS & METHOO \\
\hline 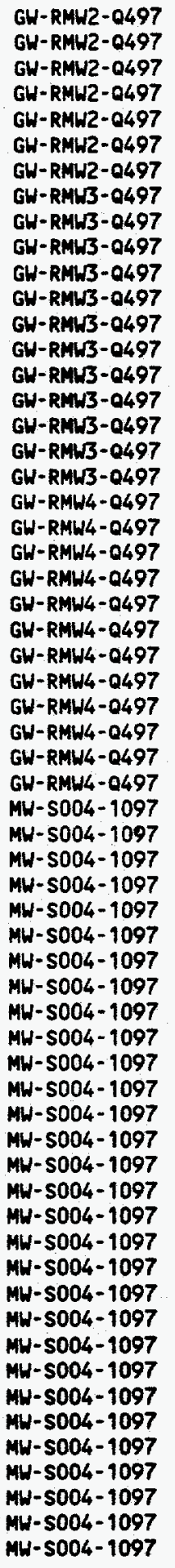 & $\begin{array}{l}12 / 16 / 97 \\
12 / 16 / 97 \\
12 / 16 / 97 \\
12 / 16 / 97 \\
12 / 16 / 97 \\
12 / 16 / 97 \\
12 / 16 / 97 \\
12 / 17 / 97 \\
12 / 17 / 97 \\
12 / 17 / 97 \\
12 / 17 / 97 \\
12 / 17 / 97 \\
12 / 17 / 97 \\
12 / 17 / 97 \\
12 / 17 / 97 \\
12 / 17 / 97 \\
12 / 17 / 97 \\
12 / 17 / 97 \\
12 / 17 / 97 \\
12 / 16 / 97 \\
12 / 16 / 97 \\
12 / 16 / 97 \\
12 / 16 / 97 \\
12 / 16 / 97 \\
12 / 16 / 97 \\
12 / 16 / 97 \\
12 / 16 / 97 \\
12 / 16 / 97 \\
12 / 16 / 97 \\
12 / 16 / 97 \\
12 / 16 / 97 \\
10 / 16 / 97 \\
10 / 16 / 97 \\
10 / 16 / 97 \\
10 / 16 / 97 \\
10 / 16 / 97 \\
10 / 16 / 97 \\
10 / 16 / 97 \\
10 / 16 / 97 \\
10 / 16 / 97 \\
10 / 16 / 97 \\
10 / 16 / 97 \\
10 / 16 / 97 \\
10 / 16 / 97 \\
10 / 16 / 97 \\
10 / 16 / 97 \\
10 / 16 / 97 \\
10 / 16 / 97 \\
10 / 16 / 97 \\
10 / 16 / 97 \\
10 / 16 / 97 \\
10 / 16 / 97 \\
10 / 16 / 97 \\
10 / 16 / 97 \\
10 / 16 / 97 \\
10 / 16 / 97 \\
10 / 16 / 97 \\
10 / 16 / 97 \\
10 / 16 / 97 \\
10 / 16 / 97 \\
10 / 16 / 97\end{array}$ & 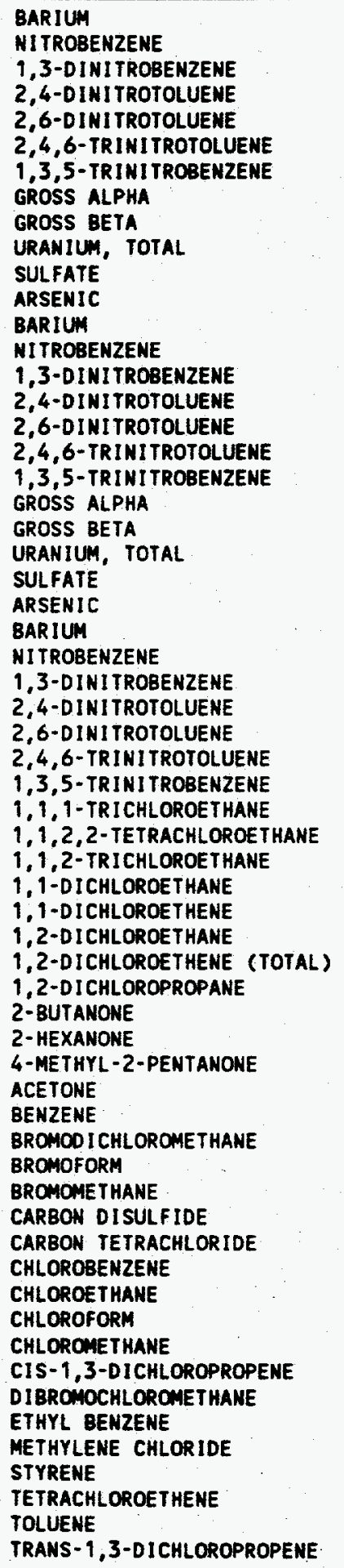 & $\begin{array}{l}408 \\
\text { ND } \\
\text { ND } \\
\text { ND } \\
\text { ND } \\
\text { ND } \\
\text { ND } \\
1.41 \\
3.83 \\
\text { ND } \\
11.0 \\
40.8 \\
597 \\
\text { ND } \\
\text { ND } \\
\text { ND } \\
\text { ND } \\
\text { ND } \\
\text { ND } \\
1.43 \\
4.36 \\
1.95 \\
31.5 \\
27.0 \\
233 \\
\text { ND } \\
\text { ND } \\
\text { ND } \\
\text { ND } \\
\text { ND } \\
\text { ND } \\
\text { ND } \\
\text { ND } \\
\text { ND } \\
\text { ND } \\
\text { ND } \\
\text { ND } \\
\text { ND } \\
\text { ND } \\
\text { ND } \\
\text { ND } \\
\text { ND } \\
\text { ND } \\
\text { ND } \\
\text { ND } \\
\text { ND } \\
\text { ND } \\
\text { ND } \\
\text { ND } \\
\text { ND } \\
\text { ND } \\
\text { ND } \\
\text { ND } \\
\text { ND } \\
\text { ND } \\
\text { ND } \\
\text { ND } \\
\text { ND } \\
\text { ND } \\
\text { ND } \\
\text { ND } \\
\text { No }\end{array}$ & $\begin{array}{l}2.0 \\
0.030 \\
0.090 \\
0.030 \\
0.010 \\
0.030 \\
0.030 \\
0.919 \\
0.872 \\
0.677 \\
0.5 \\
4.0 \\
2.0 \\
0.030 \\
0.090 \\
0.030 \\
0.010 \\
0.030 \\
0.030 \\
1.07 \\
0.871 \\
0.677 \\
1.0 \\
4.0 \\
2.0 \\
0.030 \\
0.090 \\
0.030 \\
0.010 \\
0.030 \\
0.030 \\
10 \\
10 \\
10 \\
10 \\
10 \\
10 \\
10 \\
10 \\
10 \\
10 \\
10 \\
10 \\
10 \\
10 \\
10 \\
10 \\
10 \\
10 \\
10 \\
10 \\
10 \\
10 \\
10 \\
10 \\
10 \\
10 \\
10 \\
10 \\
10 \\
10 \\
0\end{array}$ & 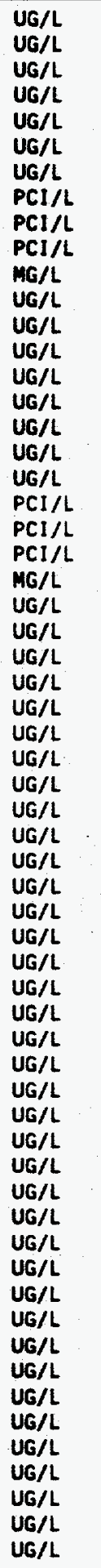 & $\begin{array}{l}\text { EPA CLP } \\
\text { USATHAMA } \\
\text { USATHAMA } \\
\text { USATHAMA } \\
\text { USATHAMA } \\
\text { USATHAMA } \\
\text { USATHAMA } \\
\text { EPA } 900.0 \\
\text { EPA } 900.0 \\
\text { ASTM } 5174-91 \\
\text { EPA } 300.0 \\
\text { EPA CLP } \\
\text { EPA CLP } \\
\text { USATHAMA } \\
\text { USATHAMA } \\
\text { USATHAMA } \\
\text { USATHAMA } \\
\text { USATHAMA } \\
\text { USATHAMA } \\
\text { EPA } 900.0 \\
\text { EPA 900.0 } \\
\text { ASTM } 5174-91 \\
\text { EPA } 300.0 \\
\text { EPA CLP } \\
\text { EPA CLP } \\
\text { USATHAMA } \\
\text { USATHAMA } \\
\text { USATHAMA } \\
\text { USATHAMA } \\
\text { USATHAMA } \\
\text { USATHAMA } \\
\text { EPA CLP } \\
\text { EPA CLP } \\
\text { EPA CLP } \\
\text { EPA CLP } \\
\text { EPA CLP } \\
\text { EPA CLP } \\
\text { EPA CLP } \\
\text { EPA CLP } \\
\text { EPA CLP } \\
\text { EPA CLP } \\
\text { EPA CLP } \\
\text { EPA CLP } \\
\text { EPA CLP } \\
\text { EPA CLP } \\
\text { EPA CLP } \\
\text { EPA CLP } \\
\text { EPA CLP } \\
\text { EPA CLP } \\
\text { EPA CLP } \\
\text { EPA CLP } \\
\text { EPA CLP } \\
\text { EPA CLP } \\
\text { EPA CLP } \\
\text { EPA CLP } \\
\text { EPA CLP } \\
\text { EPA CLP } \\
\text { EPA CLP } \\
\text { EPA CLP } \\
\text { EPA CLP } \\
\text { EPA CLP } \\
\text { SPA }\end{array}$ \\
\hline
\end{tabular}




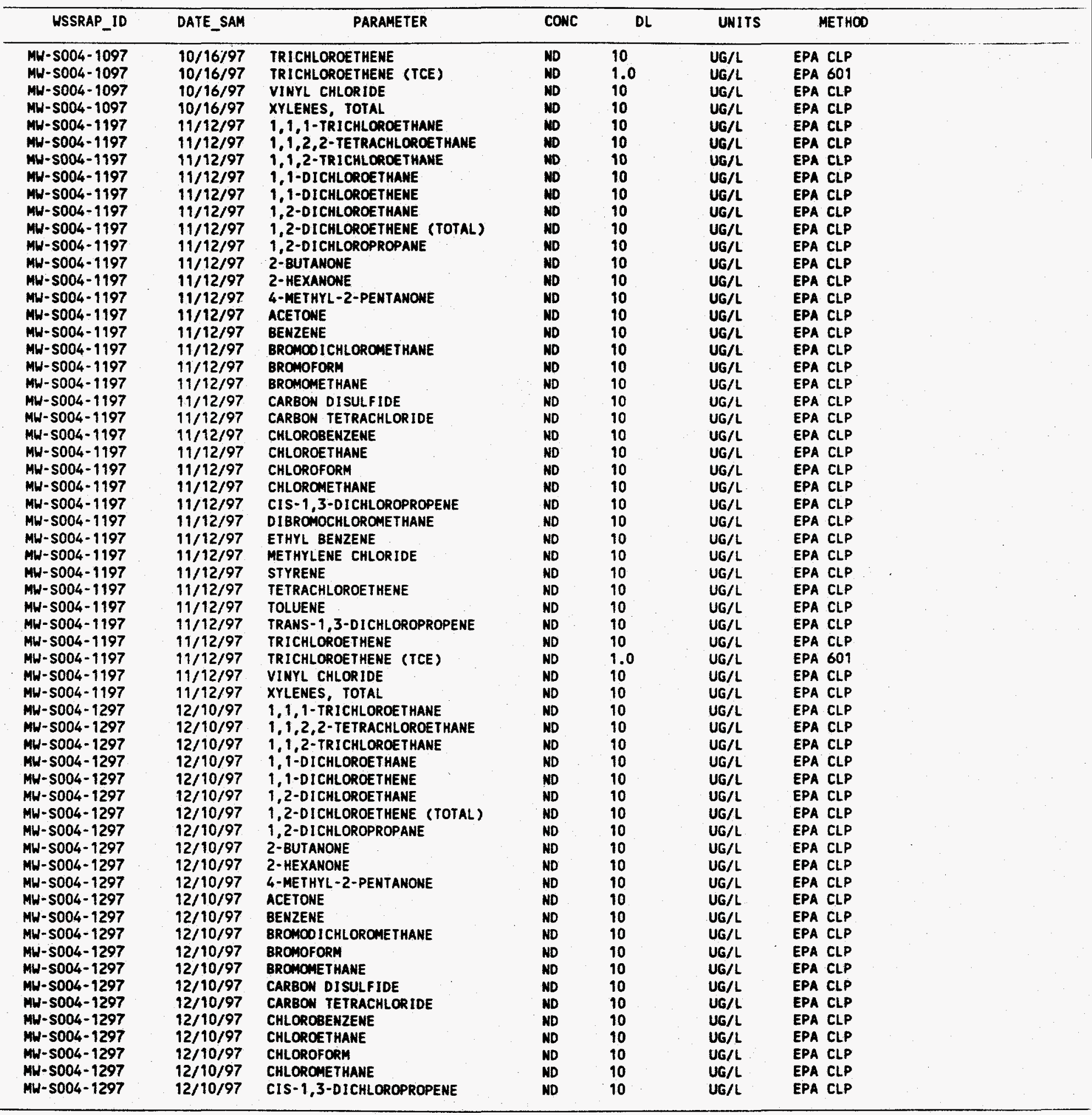




\begin{tabular}{|c|c|c|c|c|c|c|}
\hline WSSRAP_ID & DATE_SAM & PARAMETER & CONC & DL & UNITS & METHOO \\
\hline 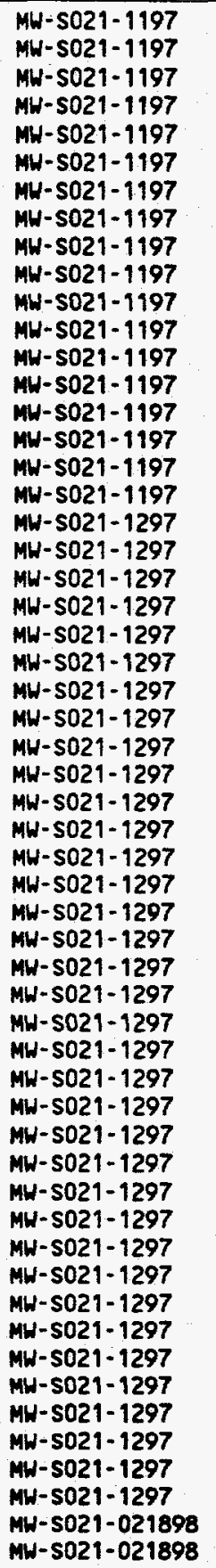 & $\begin{array}{l}11 / 12 / 97 \\
11 / 12 / 97 \\
11 / 12 / 97 \\
11 / 12 / 97 \\
11 / 12 / 97 \\
11 / 12 / 97 \\
11 / 12 / 97 \\
11 / 12 / 97 \\
11 / 12 / 97 \\
11 / 12 / 97 \\
11 / 12 / 97 \\
11 / 12 / 97 \\
11 / 12 / 97 \\
11 / 12 / 97 \\
11 / 12 / 97 \\
11 / 12 / 97 \\
11 / 12 / 97 \\
11 / 12 / 97 \\
12 / 10 / 97 \\
12 / 10 / 97 \\
12 / 10 / 97 \\
12 / 10 / 97 \\
12 / 10 / 97 \\
12 / 10 / 97 \\
12 / 10 / 97 \\
12 / 10 / 97 \\
12 / 10 / 97 \\
12 / 10 / 97 \\
12 / 10 / 97 \\
12 / 10 / 97 \\
12 / 10 / 97 \\
12 / 10 / 97 \\
12 / 10 / 97 \\
12 / 10 / 97 \\
12 / 10 / 97 \\
12 / 10 / 97 \\
12 / 10 / 97 \\
12 / 10 / 97 \\
12 / 10 / 97 \\
12 / 10 / 97 \\
12 / 10 / 97 \\
12 / 10 / 97 \\
12 / 10 / 97 \\
12 / 10 / 97 \\
12 / 10 / 97 \\
12 / 10 / 97 \\
12 / 10 / 97 \\
12 / 10 / 97 \\
12 / 10 / 97 \\
12 / 10 / 97 \\
12 / 10 / 97 \\
12 / 10 / 97 \\
12 / 10 / 97 \\
12 / 10 / 97 \\
02 / 18 / 98 \\
02 / 18 / 98\end{array}$ & 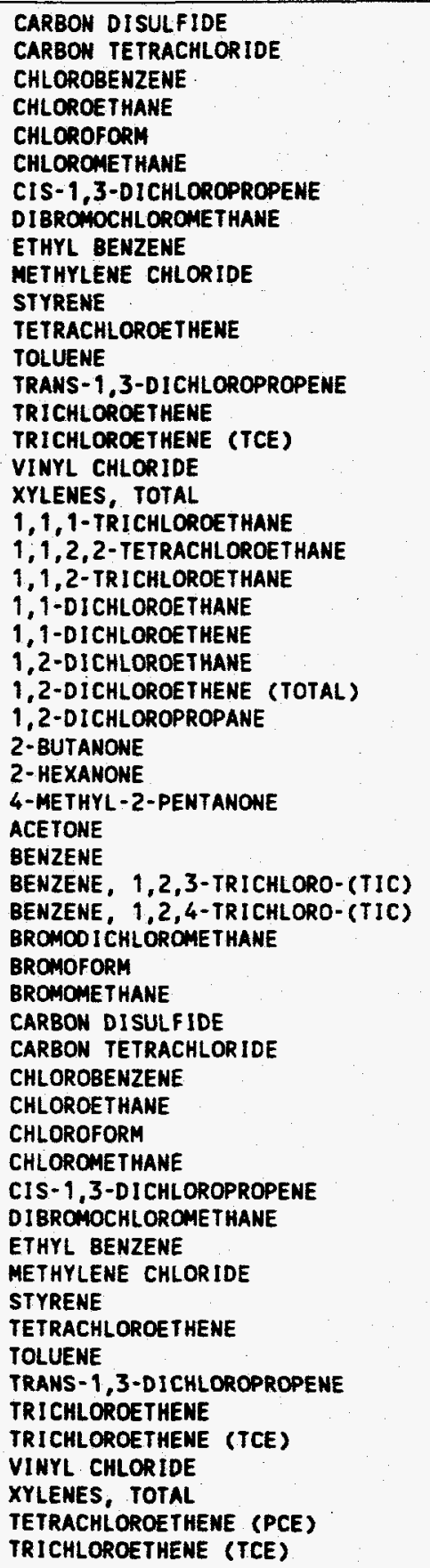 & 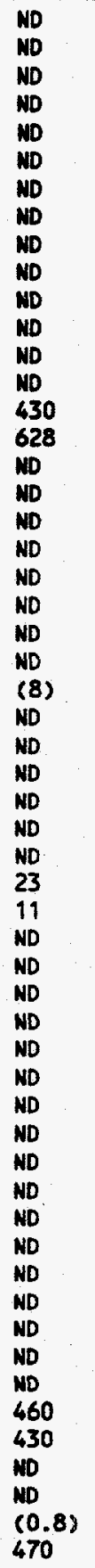 & $\begin{array}{l}10 \\
10 \\
10 \\
10 \\
10 \\
10 \\
10 \\
10 \\
10 \\
10 \\
10 \\
10 \\
10 \\
10 \\
10 \\
10 \\
10 \\
10 \\
100 \\
100 \\
10 \\
10 \\
10 \\
10 \\
10 \\
10 \\
10 \\
10 \\
10 \\
10 \\
10 \\
10 \\
10 \\
10 \\
10\end{array}$ & 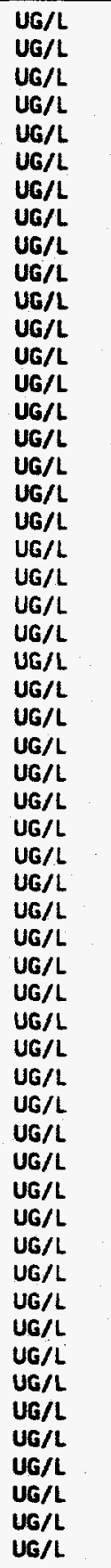 & $\begin{array}{l}\text { EPA CLP } \\
\text { EPA CLP } \\
\text { EPA CLP } \\
\text { EPA CLP } \\
\text { EPA CLP } \\
\text { EPA CLP } \\
\text { EPA CLP } \\
\text { EPA CLP } \\
\text { EPA CLP } \\
\text { EPA CLP } \\
\text { EPA CLP } \\
\text { EPA CLP } \\
\text { EPA CLP } \\
\text { EPA CLP } \\
\text { EPA CLP } \\
\text { EPA } 601 \\
\text { EPA CLP } \\
\text { EPA CLP } \\
\text { EPA CLP } \\
\text { EPA CLP } \\
\text { EPA CLP } \\
\text { EPA CLP } \\
\text { EPA CLP } \\
\text { EPA CLP } \\
\text { EPA CLP } \\
\text { EPA CLP } \\
\text { EPA CLP } \\
\text { EPA CLP } \\
\text { EPA CLP } \\
\text { EPA CLP } \\
\text { EPA CLP } \\
\text { EPA CLP } \\
\text { EPA CLP } \\
\text { EPA CLP } \\
\text { EPA CLP } \\
\text { EPA CLP } \\
\text { EPA CLP } \\
\text { EPA CLP } \\
\text { EPA CLP } \\
\text { EPA CLP } \\
\text { EPA CLP } \\
\text { EPA CLP } \\
\text { EPA CLP } \\
\text { EPA CLP } \\
\text { EPA CLP } \\
\text { EPA CLP } \\
\text { EPA CLP } \\
\text { EPA CLP } \\
\text { EPA CLP } \\
\text { EPA CLP } \\
\text { EPA CLP } \\
\text { EPA } 601 \\
\text { EPA CLP } \\
\text { EPA CLP } \\
\text { EPA } 8260 \\
\text { EPA } 8260\end{array}$ \\
\hline
\end{tabular}




\begin{tabular}{|c|c|c|c|c|c|c|}
\hline WSSRAP_ID & DATE_SAM & PARAMETER & CONC & DL & UNITS & METHOO \\
\hline 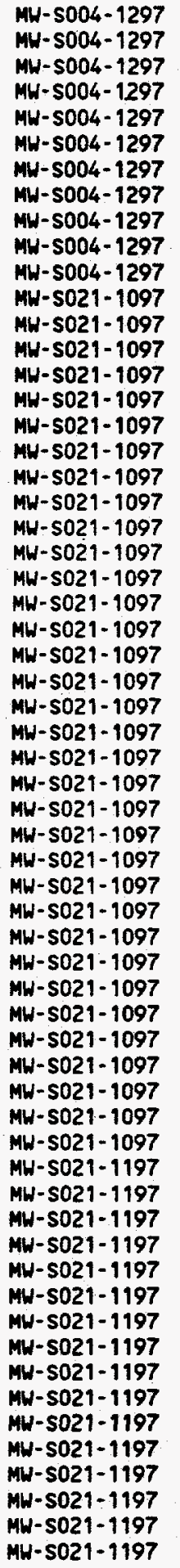 & $\begin{array}{l}12 / 10 / 97 \\
12 / 10 / 97 \\
12 / 10 / 97 \\
12 / 10 / 97 \\
12 / 10 / 97 \\
12 / 10 / 97 \\
12 / 10 / 97 \\
12 / 10 / 97 \\
12 / 10 / 97 \\
12 / 10 / 97 \\
12 / 10 / 97 \\
10 / 16 / 97 \\
10 / 16 / 97 \\
10 / 16 / 97 \\
10 / 16 / 97 \\
10 / 16 / 97 \\
10 / 16 / 97 \\
10 / 16 / 97 \\
10 / 16 / 97 \\
10 / 16 / 97 \\
10 / 16 / 97 \\
10 / 16 / 97 \\
10 / 16 / 97 \\
10 / 16 / 97 \\
10 / 16 / 97 \\
10 / 16 / 97 \\
10 / 16 / 97 \\
10 / 16 / 97 \\
10 / 16 / 97 \\
10 / 16 / 97 \\
10 / 16 / 97 \\
10 / 16 / 97 \\
10 / 16 / 97 \\
10 / 16 / 97 \\
10 / 16 / 97 \\
10 / 16 / 97 \\
10 / 16 / 97 \\
10 / 16 / 97 \\
10 / 16 / 97 \\
10 / 16 / 97 \\
10 / 16 / 97 \\
10 / 16 / 97 \\
10 / 16 / 97 \\
10 / 16 / 97 \\
10 / 16 / 97 \\
11 / 12 / 97 \\
11112 / 97 \\
11 / 12 / 97 \\
11 / 12 / 97 \\
11 / 12 / 97 \\
11 / 12 / 97 \\
111112 / 97 \\
11 / 12 / 97 \\
11 / 12 / 97 \\
11 / 12 / 97 \\
11 / 12 / 97 \\
11 / 12 / 97 \\
11 / 112 / 97 \\
11 / 12 / 97 \\
11 / 12 / 97 \\
11 / 12 / 97\end{array}$ & 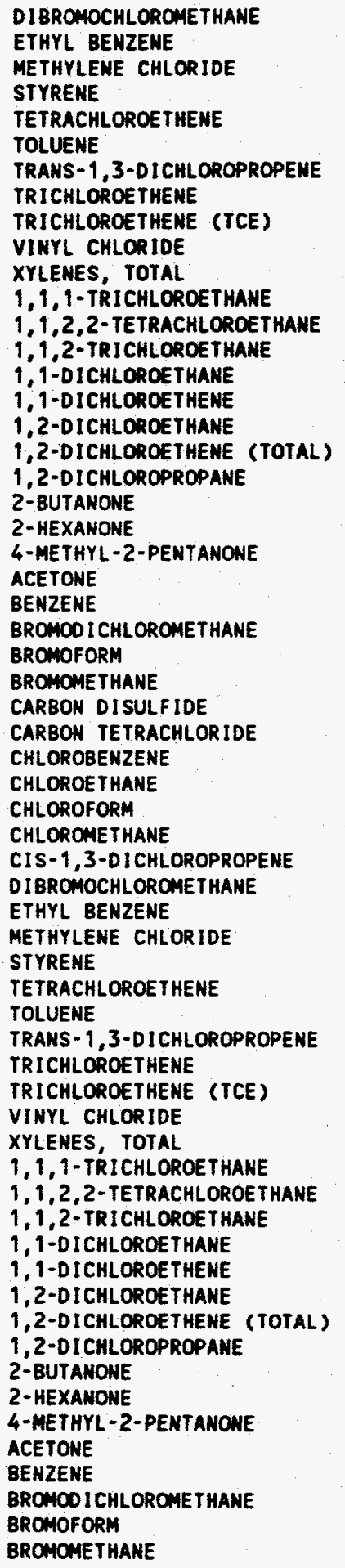 & 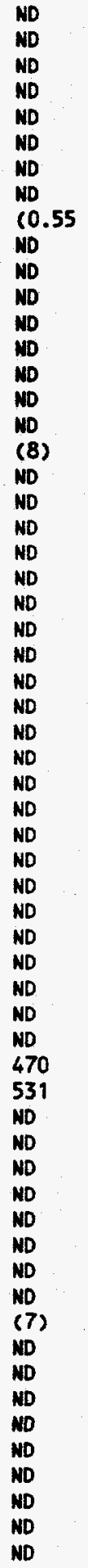 & $\begin{array}{l}10 \\
10 \\
10 \\
10 \\
10 \\
10 \\
10 \\
10 \\
1.0 \\
10 \\
10 \\
10 \\
10 \\
10 \\
10 \\
10 \\
10 \\
10 \\
10 \\
10 \\
10 \\
10 \\
10 \\
10 \\
10 \\
10 \\
10 \\
10 \\
10 \\
10 \\
10 \\
10 \\
10 \\
10 \\
10 \\
10 \\
10 \\
10 \\
10 \\
10 \\
10 \\
100 \\
100 \\
10 \\
10 \\
10 \\
10 \\
10 \\
10 \\
10 \\
10 \\
10 \\
10 \\
10 \\
10 \\
10 \\
10 \\
10 \\
10 \\
10 \\
10\end{array}$ & 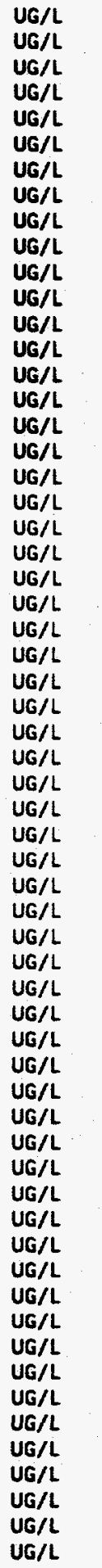 & 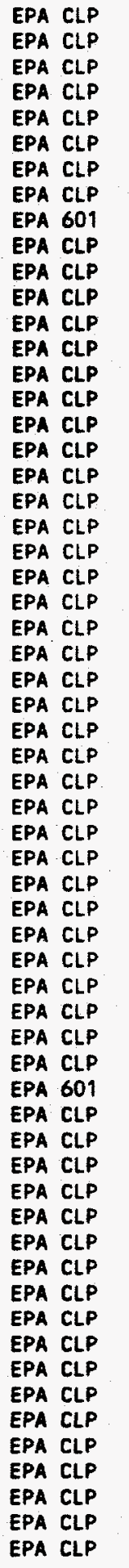 \\
\hline
\end{tabular}


is

nition

itmonim

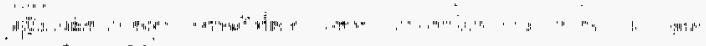

Hathond

(n)

and

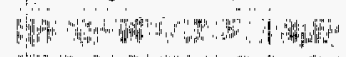

Ifit

ifind

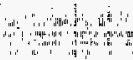

\section{SURFACE WATER}

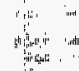

int

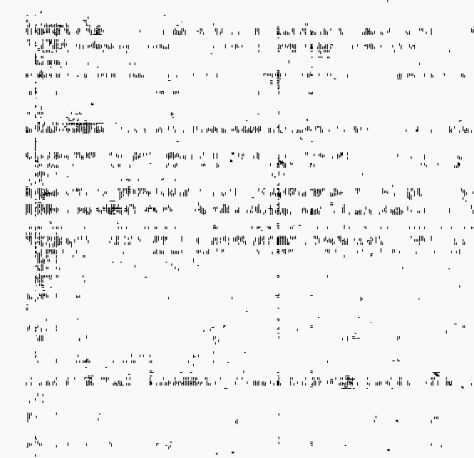

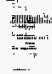

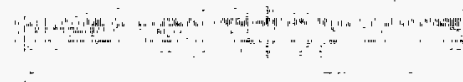
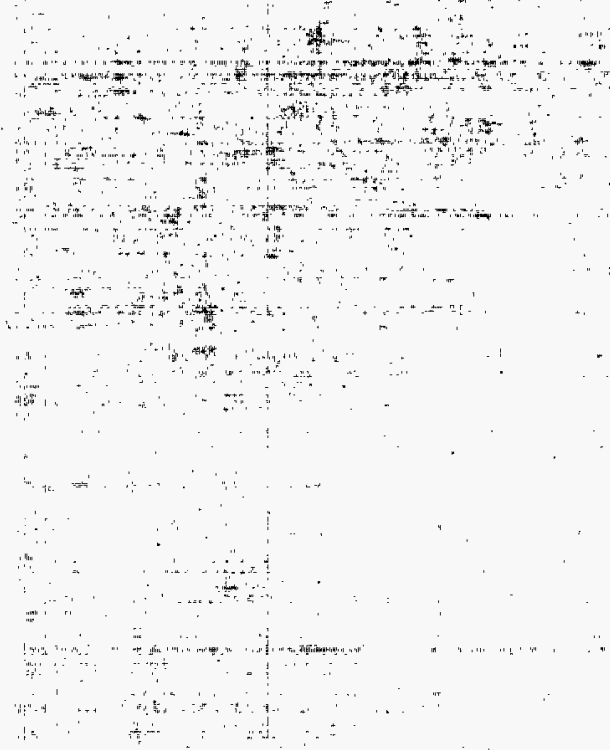

Pit 
SURFACE WATER

DATA MERGED DURING FIRST QUARTER 1998

\begin{tabular}{|c|c|c|c|c|c|c|}
\hline USSRAP_ID & DATE_SAM & PARAMETER & conc & $D L$ & UNITS & METHOD \\
\hline 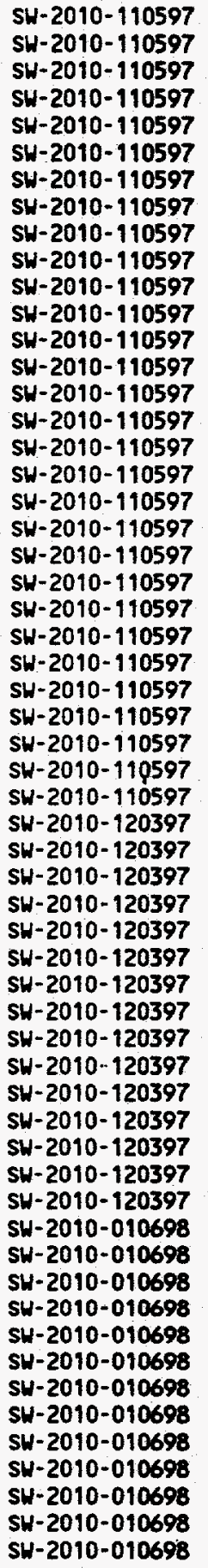 & $\begin{array}{l}11 / 05 / 97 \\
11 / 05 / 97 \\
11 / 05 / 97 \\
11 / 05 / 97 \\
11 / 05 / 97 \\
11 / 05 / 97 \\
11 / 05 / 97 \\
11 / 05 / 97 \\
11 / 05 / 97 \\
11 / 05 / 97 \\
11 / 05 / 97 \\
11 / 05 / 97 \\
11 / 05 / 97 \\
11 / 05 / 97 \\
11 / 05 / 97 \\
11 / 05 / 97 \\
11 / 05 / 97 \\
11 / 05 / 97 \\
11 / 05 / 97 \\
11 / 05 / 97 \\
11 / 05 / 97 \\
11 / 05 / 97 \\
11 / 05 / 97 \\
11 / 05 / 97 \\
11 / 05 / 97 \\
11 / 05 / 97 \\
11 / 05 / 97 \\
11 / 05 / 97 \\
11 / 05 / 97 \\
11 / 05 / 97 \\
12 / 03 / 97 \\
12 / 03 / 97 \\
12 / 03 / 97 \\
12 / 03 / 97 \\
12 / 03 / 97 \\
12 / 03 / 97 \\
12 / 03 / 97 \\
12 / 03 / 97 \\
12 / 03 / 97 \\
12 / 03 / 97 \\
12 / 03 / 97 \\
12 / 03 / 97 \\
12 / 03 / 97 \\
12 / 03 / 97 \\
12 / 03 / 97 \\
01 / 06 / 98 \\
011 / 06 / 98 \\
01 / 06 / 98 \\
01 / 06 / 98 \\
01 / 06 / 98 \\
011 / 06 / 98 \\
01 / 06 / 98 \\
01 / 06 / 98 \\
01 / 06 / 98 \\
01 / 06 / 98 \\
01 / 06 / 98 \\
01 / 06 / 98 \\
01 / 06 / 98\end{array}$ & 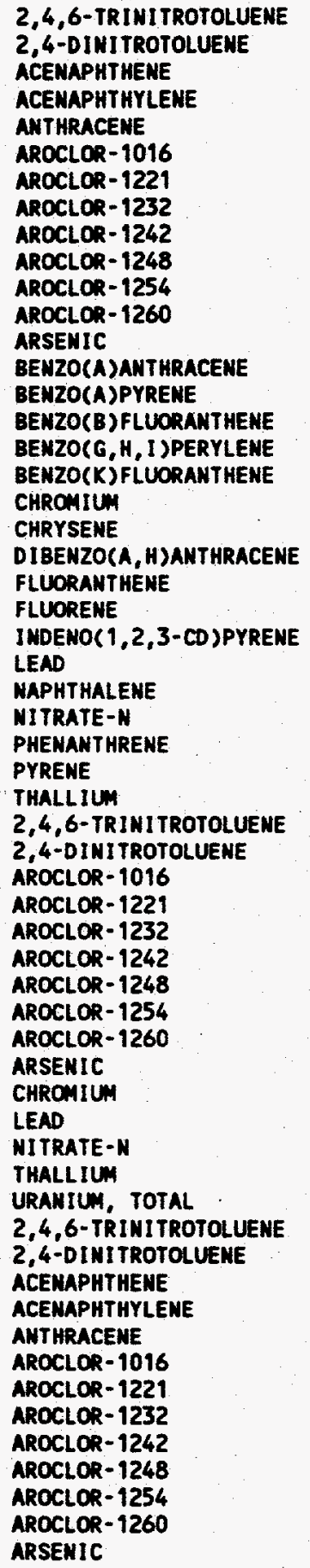 & 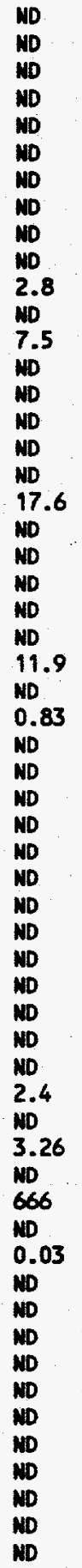 & 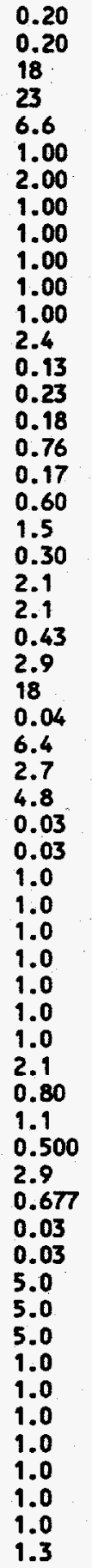 & $\begin{array}{l}\text { UG/L } \\
\text { UG/L } \\
\text { UG/L } \\
\text { UG/L } \\
\text { UG/L } \\
\text { UG/L } \\
\text { UG/L } \\
\text { UG/L } \\
\text { UG/L } \\
\text { UG/L } \\
\text { UG/L } \\
\text { UG/L } \\
\text { UG/L } \\
\text { UG/L } \\
\text { UG/L } \\
\text { UG/L } \\
\text { UG/L } \\
\text { UG/L } \\
\text { UG/L } \\
\text { UG/L } \\
\text { UG/L } \\
\text { UG/L } \\
\text { UG/L } \\
\text { UG/L } \\
\text { UG/L } \\
\text { UG/L } \\
\text { UG/L } \\
\text { UG/L } \\
\text { UG/L } \\
\text { UG/L } \\
\text { UG/L } \\
\text { UG/L } \\
\text { UG/L } \\
\text { UG/L } \\
\text { UG/L } \\
\text { UG/L } \\
\text { UG/L } \\
\text { UG/L } \\
\text { UG/L } \\
\text { UG/L } \\
\text { UG/L } \\
\text { UG/L } \\
\text { UG/L } \\
\text { UG/L } \\
P C I / L \\
\text { UG/L } \\
\text { UG/L } \\
\text { UG/L } \\
\text { UG/L } \\
\text { UG/L } \\
\text { UG/L } \\
\text { UG/L } \\
\text { UG/L } \\
\text { UG/L } \\
\text { UG/L } \\
\text { UG/L } \\
\text { UG/L } \\
\text { UG/L }\end{array}$ & $\begin{array}{l}\text { EPA } 8330 \\
\text { EPA } 8330 \\
\text { EPA } 8310 \\
\text { EPA } 8310 \\
\text { EPA } 8310 \\
\text { EPA } 8081 \\
\text { EPA } 8081 \\
\text { EPA } 8081 \\
\text { EPA } 8081 \\
\text { EPA } 8081 \\
\text { EPA } 8081 \\
\text { EPA } 8081 \\
\text { EPA CLP } \\
\text { EPA } 8310 \\
\text { EPA } 8310 \\
\text { EPA } 8310 \\
\text { EPA } 8310 \\
\text { EPA } 8310 \\
\text { EPA CLP } \\
\text { EPA } 8310 \\
\text { EPA } 8310 \\
\text { EPA } 8310 \\
\text { EPA } 8310 \\
\text { EPA } 8310 \\
\text { EPA CLP } \\
\text { EPA } 8310 \\
\text { EPA } 353.2 \\
\text { EPA } 8310 \\
\text { EPA } 8310 \\
\text { EPA CLP } \\
\text { USATHAMA } \\
\text { USATHAMA } \\
\text { EPA } 8080 A \\
\text { EPA } 8080 A \\
\text { EPA } 8080 A \\
\text { EPA } 8080 A \\
\text { EPA } 8080 A \\
\text { EPA } 8080 A \\
\text { EPA } 8080 A \\
\text { EPA CLP } \\
\text { EPA CLP } \\
\text { EPA CLP } \\
\text { EPA } 353.1 \\
\text { EPA CLP } \\
\text { ASTM } 5174-91 \\
\text { USATHAMA } \\
\text { USATHAMA } \\
\text { EPA } 8310 \\
\text { EPA } 8310 \\
\text { EPA } 8310 \\
\text { EPA } 8080 A \\
\text { EPA } 8080 A \\
\text { EPA } 8080 A \\
\text { EPA } 8080 A \\
\text { EPA } 8080 A \\
\text { EPA } 8080 A \\
\text { EPA } 8080 A \\
\text { EPA CLP } \\
\end{array}$ \\
\hline
\end{tabular}




\begin{tabular}{|c|c|c|c|c|c|c|}
\hline WSSRAP_ID & DATE_SAM & PARAMETER & CONC & DL & UNITS & METHOO \\
\hline $\begin{array}{l}\text { SW-2010-010698 } \\
\text { SW-2010-010698 } \\
\text { SW-2010-010698 } \\
\text { SW-2010-010698 } \\
\text { SW-2010-010698 } \\
\text { SW-2010-010698 } \\
\text { SW-2010-010698 } \\
\text { SW-2C10-010698 } \\
\text { SW-2010-010698 } \\
\text { SW-2010-010698 } \\
\text { SW-2010-010698 } \\
\text { SW-2010-010698 } \\
\text { SW-2010-010698 } \\
\text { SW-2010-010698 } \\
\text { SW-2010-010698 } \\
\text { SW-2010-010698 } \\
\text { SW-2010-010698 } \\
\text { SW-2010-010698 } \\
\text { SW-2010-010698 } \\
\text { SW-2010-010698 } \\
\text { SW-2010-010698 } \\
\text { SW-2010-010698 } \\
\text { SW-2010-010698 } \\
\text { SW-2010-010698 } \\
\text { SW-2010-021098 } \\
\text { SW-2010-030998 } \\
\text { SW-2017-110397 } \\
\text { SW-2017-110397 } \\
\text { SW-2017-1110397 } \\
\text { SW-2017-110397 } \\
\text { SW-2017-110397 } \\
\text { SW-2017-120397 } \\
\text { SW-2017-120397 } \\
\text { SW-2017-120397 } \\
\text { SW-2017-120397 } \\
\text { SW-2017-120397 } \\
\text { SW-2017-010698 } \\
\text { SW-2017-010698 } \\
\text { SW-2017-010698 } \\
\text { SW-2017-010698 } \\
\text { SW-2017-010698 } \\
\text { SW-2017-021098 } \\
\text { SW-2017-030998 } \\
\text { SW-2018-0111298 } \\
\text { SW-2018-0111298 } \\
\text { SW-2018-0112988 } \\
\text { SW-2018-011298 } \\
\text { SW-2018-2018-0111298 } \\
\text { SW-2018-0111298 } \\
\text { SW-2018-0112988 } \\
\text { SW-2018-011298 } \\
\text { SW-2018-011298 } \\
\text { SW-2018-0112988 } \\
\text { SW-2018-0112988 } \\
\text { SW-2018-0111298 } \\
\text { SW-2018-011298 } \\
\text { SW-2018-011298 }\end{array}$ & $\begin{array}{l}01 / 06 / 98 \\
01 / 06 / 98 \\
01 / 06 / 98 \\
01 / 06 / 98 \\
01 / 06 / 98 \\
01 / 06 / 98 \\
01 / 06 / 98 \\
01 / 06 / 98 \\
01 / 06 / 98 \\
01 / 06 / 98 \\
01 / 06 / 98 \\
01 / 06 / 98 \\
01 / 06 / 98 \\
01 / 06 / 98 \\
01 / 06 / 98 \\
01 / 06 / 98 \\
01 / 06 / 98 \\
01 / 06 / 98 \\
01 / 06 / 98 \\
01 / 06 / 98 \\
01 / 06 / 98 \\
01 / 06 / 98 \\
01 / 06 / 98 \\
01 / 06 / 98 \\
02 / 10 / 98 \\
03 / 09 / 98 \\
11 / 03 / 97 \\
11 / 03 / 97 \\
11 / 03 / 97 \\
11 / 03 / 97 \\
11 / 03 / 97 \\
12 / 03 / 97 \\
11 / 03 / 97 \\
12 / 03 / 97 \\
12 / 03 / 97 \\
12 / 03 / 97 \\
01 / 06 / 98 \\
01 / 06 / 98 \\
01 / 06 / 98 \\
01 / 06 / 98 \\
01 / 06 / 98 \\
02 / 10 / 98 \\
03 / 09 / 98 \\
01 / 12 / 98 \\
01 / 12 / 98 \\
01 / 12 / 98 \\
01 / 12 / 98 \\
01 / 12 / 98 \\
01 / 12 / 98 \\
01 / 12 / 98 \\
01 / 12 / 98 \\
01 / 12 / 98 \\
01 / 12 / 98 \\
01 / 12 / 98 \\
01 / 12 / 98 \\
01 / 12 / 98 \\
01 / 12 / 98 \\
01 / 12 / 98 \\
01 / 12 / 98 \\
01 / 12 / 98 \\
01 / 12 / 98\end{array}$ & 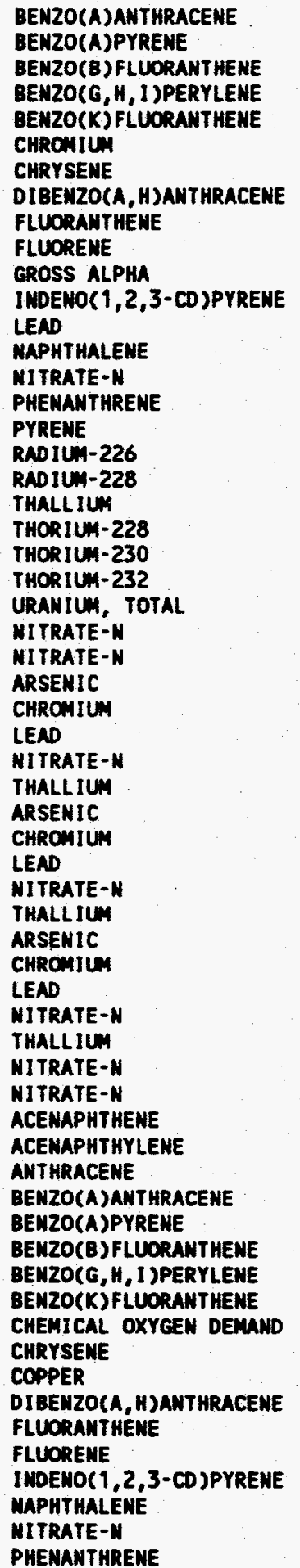 & 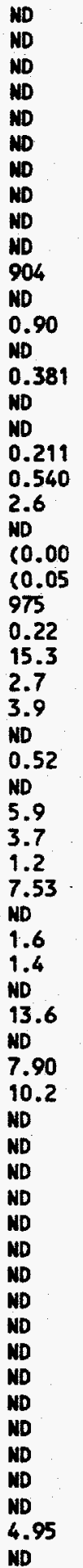 & $\begin{array}{l}5.0 \\
5.0 \\
5.0 \\
5.0 \\
5.0 \\
0.60 \\
5.0 \\
5.0 \\
5.0 \\
5.0 \\
1.98 \\
5.0 \\
0.90 \\
5.0 \\
0.050 \\
5.0 \\
5.0 \\
0.023 \\
0.319 \\
1.8 \\
0.057 \\
0.180 \\
0.074 \\
13.5 \\
0.050 \\
1.00 \\
2.4 \\
0.60 \\
2.9 \\
0.02 \\
4.8 \\
2.1 \\
0.80 \\
1.1 \\
0.500 \\
2.9 \\
1.3 \\
0.60 \\
0.90 \\
1.00 \\
1.8 \\
1.00 \\
1.00 \\
5.0 \\
5.0 \\
5.0 \\
5.0 \\
5.0 \\
5.0 \\
5.0 \\
5.0 \\
5.00 \\
5.0 \\
3.1 \\
5.0 \\
5.0 \\
5.0 \\
5.0 \\
5.0 \\
1.00 \\
5.0\end{array}$ & 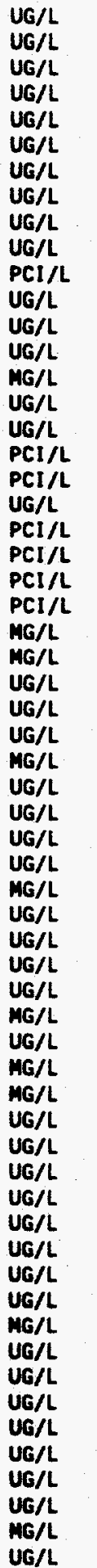 & $\begin{array}{l}\text { EPA } 8310 \\
\text { EPA } 8310 \\
\text { EPA } 8310 \\
\text { EPA } 8310 \\
\text { EPA } 8310 \\
\text { EPA CLP } \\
\text { EPA } 8310 \\
\text { EPA } 8310 \\
\text { EPA } 8310 \\
\text { EPA } 8310 \\
\text { EPA } 900.0 \\
\text { EPA } 8310 \\
\text { EPA CLP } \\
\text { EPA } 8310 \\
\text { EPA } 353.1 \\
\text { EPA } 8310 \\
\text { EPA } 8310 \\
\text { EPA } 904.0 \\
\text { EPA } 904.0 \\
\text { EPA CLP } \\
\text { MAS- } 15-3004 \\
\text { NAS- HS-3004 } \\
\text { MAS- KS-3004 } \\
\text { ASTM } 5174-91 \\
\text { EPA } 353.1 \\
\text { EPA } 353.1 \\
\text { EPA CLP } \\
\text { EPA CLP } \\
\text { EPA CLP } \\
\text { EPA } 353.2 \\
\text { EPA CLP } \\
\text { EPA CLP } \\
\text { EPA CLP } \\
\text { EPA CLP } \\
\text { EPA } 353.1 \\
\text { EPA CLP } \\
\text { EPA CLP } \\
\text { EPA CLP } \\
\text { EPA CLP } \\
\text { EPA } 353.1 \\
\text { EPA CLP } \\
\text { EPA } 353.1 \\
\text { EPA } 353.1 \\
\text { EPA } 8310 \\
\text { EPA } 8310 \\
\text { EPA } 8310 \\
\text { EPA } 8310 \\
\text { EPA } 8310 \\
\text { EPA } 8310 \\
\text { EPA } 8310 \\
\text { EPA } 8310 \\
\text { EPA } 410.4 \\
\text { EPA } 8310 \\
\text { EPA CLP } \\
\text { EPA } 8310 \\
\text { EPA } 8310 \\
\text { EPA } 8310 \\
\text { EPA } 8310 \\
\text { EPA } 8310 \\
\text { EPA } 353.1 \\
\text { EPA } 8310 \\
\end{array}$ \\
\hline
\end{tabular}




\begin{tabular}{|c|c|c|c|c|c|c|}
\hline WSSRAP_ID & DATE_SAM & PARAMETER & conc & $D L$ & UNITS & METHOD \\
\hline 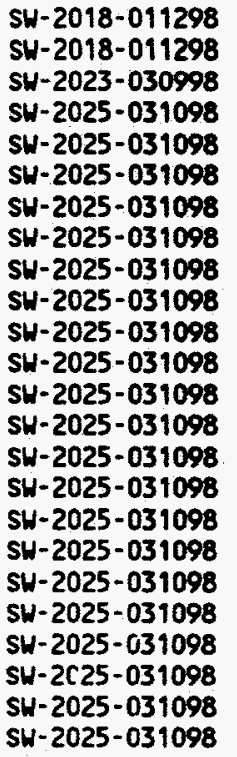 & $\begin{array}{l}01 / 12 / 98 \\
01 / 12 / 98 \\
03 / 09 / 98 \\
03 / 10 / 98 \\
03 / 10 / 98 \\
03 / 10 / 98 \\
03 / 10 / 98 \\
03 / 10 / 98 \\
03 / 10 / 98 \\
03 / 10 / 98 \\
03 / 10 / 98 \\
03 / 10 / 98 \\
03 / 10 / 98 \\
03 / 10 / 98 \\
03 / 10 / 98 \\
03 / 10 / 98 \\
03 / 10 / 98 \\
03 / 10 / 98 \\
03 / 10 / 98 \\
03 / 10 / 98 \\
03 / 10 / 98 \\
03 / 10 / 98 \\
03 / 10 / 98 \\
03 / 10 / 98\end{array}$ & $\begin{array}{l}\text { PYRENE } \\
\text { ZINC } \\
\text { MITRATE-M } \\
\text { ARSENIC } \\
\text { CHEMICAL OXYGEN DEMAND } \\
\text { CHLORIDE } \\
\text { CHROMILM } \\
\text { CYANIDE, MMEMABLE } \\
\text { FLUORIDE } \\
\text { GROSS ALPHA } \\
\text { GROSS BETA } \\
\text { LEAD } \\
\text { MANGANESE } \\
\text { MERCURY } \\
\text { MITRATE-N } \\
\text { RADIUM-226 } \\
\text { RADIUN-228 } \\
\text { SELENIUM } \\
\text { SULFATE } \\
\text { THORIUM-228 } \\
\text { THORIUM-230 } \\
\text { THORIUM-232 } \\
\text { TOTAL SUSPENDED SOLIDS } \\
\text { URANIUM, TOTAL }\end{array}$ & $\begin{array}{l}\text { ND } \\
117 \\
8.44 \\
\text { ND } \\
\text { ND } \\
0.61 \\
1.8 \\
\text { ND } \\
0.26 \\
0.591 \\
1.51 \\
\text { ND } \\
3.7 \\
\text { ND } \\
4.30 \\
C 0.04 \\
C 0.13 \\
\text { ND } \\
11.1 \\
C 0.04 \\
C 0.04 \\
C 0.04 \\
3.0 \\
N D\end{array}$ & $\begin{array}{l}5.0 \\
1.3 \\
0.50 \\
1.3 \\
5.00 \\
0.20 \\
0.60 \\
5.00 \\
0.10 \\
0.359 \\
0.768 \\
0.90 \\
0.30 \\
0.10 \\
1.00 \\
0.080 \\
0.297 \\
2.2 \\
0.50 \\
0.076 \\
0.070 \\
0.058 \\
1.00 \\
0.677\end{array}$ & $\begin{array}{l}U G / L \\
U G / L \\
M G / L \\
U G / L \\
M G / L \\
M G / L \\
U G / L \\
U G / L \\
M G / L \\
P C I / L \\
P C I / L \\
U G / L \\
U G / L \\
U G / L \\
M G / L \\
P C I / L \\
P C I / L \\
U G / L \\
M G / L \\
P C I / L \\
P C I / L \\
P C I / L \\
M G / L \\
P C I / L\end{array}$ & $\begin{array}{l}\text { EPA } 8310 \\
\text { EPA CLP } \\
\text { EPA } 353.1 \\
\text { EPA } 200.7 \\
\text { EPA } 410.4 \\
\text { EPA } 300.0 \\
\text { EPA } 200.7 \\
\text { EPA } 9010 \\
\text { EPA } 300.0 \\
\text { EPA } 900.0 \\
\text { EPA } 900.0 \\
\text { EPA } 200.7 \\
\text { EPA } 200.7 \\
\text { EPA } 245.1 \\
\text { EPA } 353.1 \\
\text { EPA } 904.0 \\
\text { EPA } 904.0 \\
\text { EPA } 200.7 \\
\text { EPA } 300.0 \\
\text { NAS-NS-3004 } \\
\text { NAS-NS-3004 } \\
\text { NAS-NS-3004 } \\
\text { EPA } 160.2 \\
\text { ASTM } 5174-91\end{array}$ \\
\hline
\end{tabular}




\section{DOWNSTREAM SURFACE WATER URANIUM RESULTS}

\begin{tabular}{|c|c|c|c|c|}
\hline WSSRAP ID & DATE & PARAMETER & CONCENTRATION & UNITS \\
\hline SW-2001-Q198 & $2 / 19 / 98$ & Uranium, Total & 1.49 & $\mathrm{pCi} /$ \\
\hline SW-2002-Q198 & $2 / 19 / 98$ & Uranium, Total & 62.39 & $\mathrm{pCi} /$ \\
\hline SW-2003-Q198 & $2 / 19 / 98$ & Uranium, Total & 11.92 & $\mathrm{pCi} /$ \\
\hline SW-2004-Q198 & $2 / 19 / 98$ & Uranium, Total & 13.81 & $\mathrm{pCi} /$ \\
\hline SW-2005-Q198 & $2 / 19 / 98$ & Uranium, Total & 42.28 & $\mathrm{pCi} /$ \\
\hline SW-2012-Q198 & $2 / 19 / 98$ & Uranium, Total & 8.28 & $\mathrm{pCi} /$ \\
\hline SW-2016-Q198 & $2 / 19 / 98$ & Uranium, Total & 1.63 & $\mathrm{pCi} /$ \\
\hline
\end{tabular}




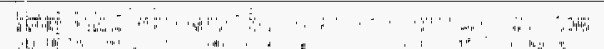

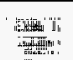

Jand

ind

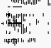

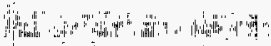

(4)

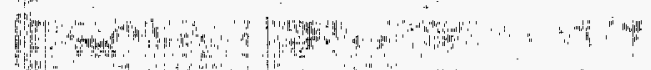
iting

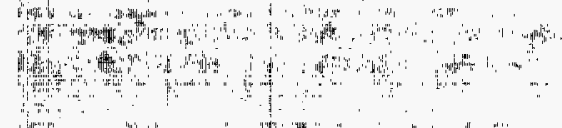

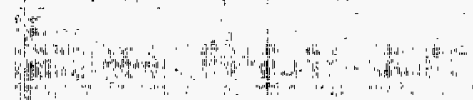

is.".

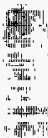

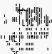

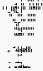

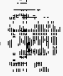

\section{SPRINGS}

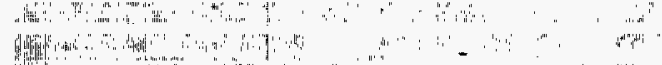

in

, inin.

种情

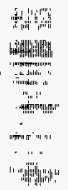

, ind

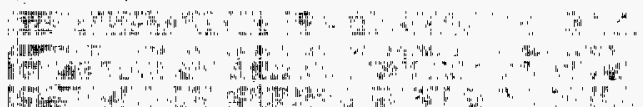

(1)

tit

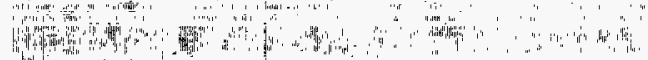

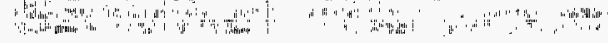

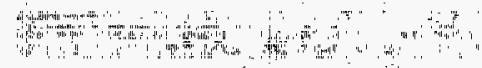

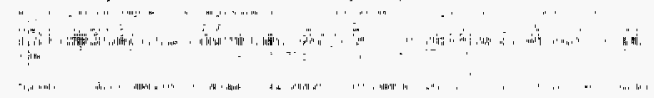

H.in. , wi.

titur:
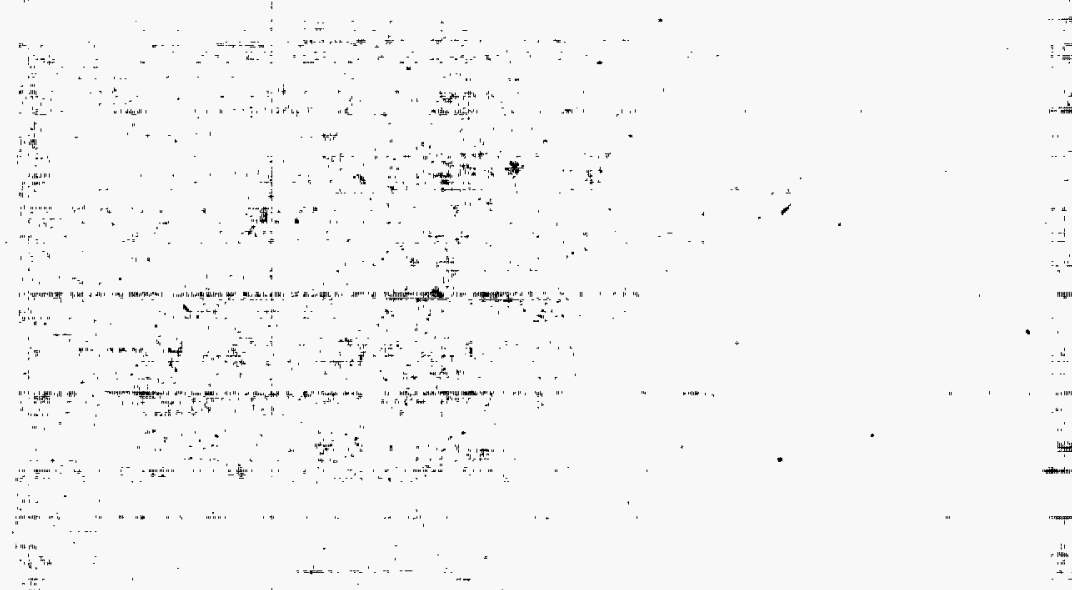

,

(n)

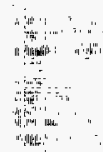




\begin{tabular}{|c|c|c|c|c|c|c|}
\hline-10 & DATE_SAM & PARAMETER & CONC & DL & UNITS & METHOD \\
\hline 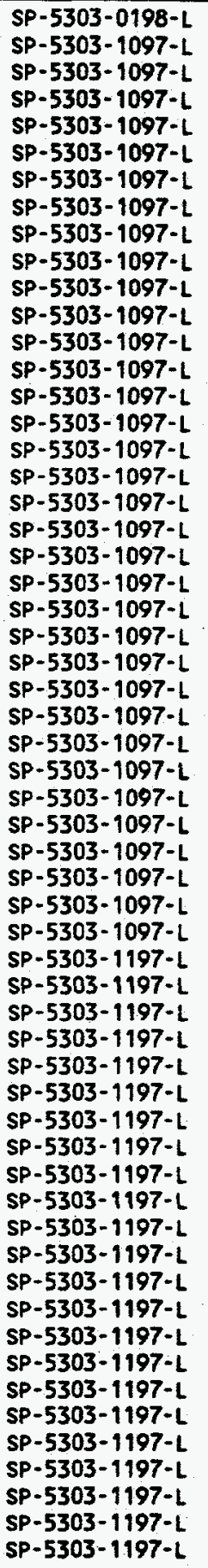 & $\begin{array}{l}01 / 15 / 98 \\
10 / 29 / 97 \\
10 / 29 / 97 \\
10 / 29 / 97 \\
10 / 29 / 97 \\
10 / 29 / 97 \\
10 / 29 / 97 \\
10 / 29 / 97 \\
10 / 29 / 97 \\
10 / 29 / 97 \\
10 / 29 / 97 \\
10 / 29 / 97 \\
10 / 29 / 97 \\
10 / 29 / 97 \\
10 / 29 / 97 \\
10 / 29 / 97 \\
10 / 29 / 97 \\
10 / 29 / 97 \\
10 / 29 / 97 \\
10 / 29 / 97 \\
10 / 29 / 97 \\
10 / 29 / 97 \\
10 / 29 / 97 \\
10 / 29 / 97 \\
10 / 29 / 97 \\
10 / 29 / 97 \\
10 / 29 / 97 \\
10 / 29 / 97 \\
10 / 29 / 97 \\
10 / 29 / 97 \\
10 / 29 / 97 \\
10 / 29 / 97 \\
10 / 29 / 97 \\
10 / 29 / 97 \\
10 / 29 / 97 \\
11 / 20 / 97 \\
11 / 20 / 97 \\
11 / 20 / 97 \\
11 / 20 / 97 \\
11 / 20 / 97 \\
11 / 20 / 97 \\
11 / 20 / 97 \\
11 / 20 / 97 \\
11 / 20 / 97 \\
11 / 20 / 97 \\
11 / 20 / 97 \\
111 / 20 / 97 \\
11 / 20 / 97 \\
11 / 20 / 97 \\
11 / 20 / 97 \\
111 / 20 / 97 \\
11 / 20 / 97 \\
11 / 20 / 97 \\
11 / 20 / 97 \\
11 / 20 / 97 \\
11 / 20 / 97 \\
11 / 20 / 97 \\
11 / 20 / 97\end{array}$ & 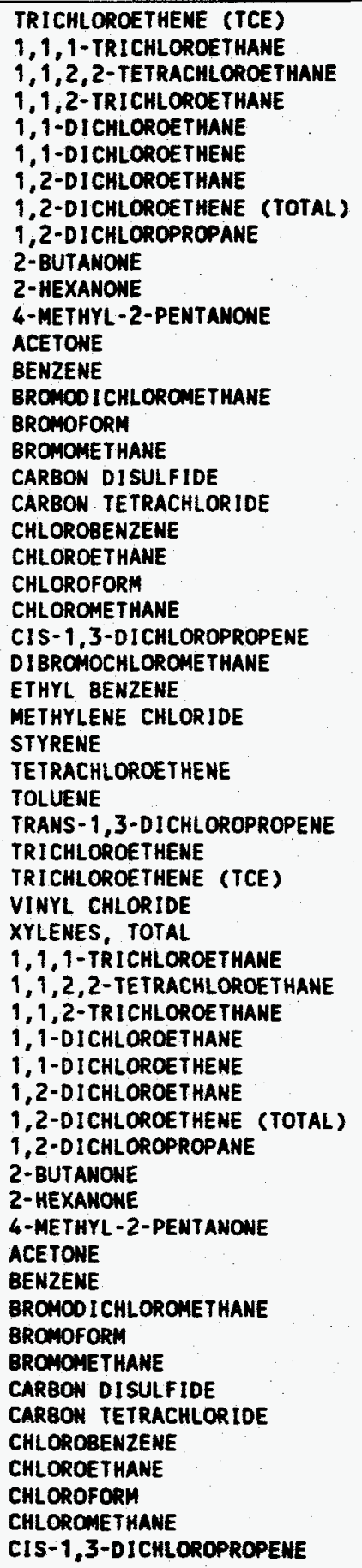 & $\begin{array}{l}\text { ND } \\
\text { ND } \\
\text { ND } \\
\text { ND } \\
\text { ND } \\
\text { ND } \\
\text { ND } \\
\text { ND } \\
\text { ND } \\
\text { ND } \\
\text { ND } \\
\text { ND } \\
\text { ND } \\
\text { ND } \\
\text { ND } \\
\text { ND } \\
\text { ND } \\
\text { ND } \\
\text { ND } \\
\text { ND } \\
\text { ND } \\
\text { ND } \\
\text { ND } \\
\text { ND } \\
\text { ND } \\
\text { ND } \\
\text { ND } \\
\text { ND } \\
\text { ND } \\
\text { ND } \\
\text { ND } \\
\text { ND } \\
\text { ND } \\
\text { ND } \\
\text { ND } \\
\text { ND } \\
\text { ND } \\
\text { ND } \\
\text { ND } \\
\text { ND } \\
\text { ND } \\
\text { ND } \\
\text { ND } \\
\text { ND } \\
\text { ND } \\
\text { ND } \\
\text { ND } \\
\text { ND } \\
\text { ND } \\
\text { ND } \\
\text { ND } \\
\text { ND } \\
\text { ND } \\
\text { ND } \\
\text { ND } \\
\text { ND } \\
\text { ND } \\
\text { ND }\end{array}$ & $\begin{array}{l}1 \\
10 \\
10 \\
10 \\
10 \\
10 \\
10 \\
10 \\
10 \\
10 \\
10 \\
10 \\
10 \\
10 \\
10 \\
10 \\
10 \\
10 \\
10 \\
10 \\
10 \\
10 \\
10 \\
10 \\
10 \\
10 \\
10 \\
10 \\
10 \\
10 \\
10 \\
10 \\
1.0 \\
10 \\
10 \\
10 \\
10 \\
10 \\
10 \\
10 \\
10 \\
10 \\
10 \\
10 \\
10 \\
10 \\
10 \\
10 \\
10 \\
10 \\
10 \\
10 \\
10 \\
10 \\
10 \\
10 \\
10 \\
10\end{array}$ & $\begin{array}{l}U G / L \\
U G / L \\
U G / L \\
U G / L \\
U G / L \\
U G / L \\
U G / L \\
U G / L \\
U G / L \\
U G / L \\
U G / L \\
U G / L \\
U G / L \\
U G / L \\
U G / L \\
U G / L \\
U G / L \\
U G / L \\
U G / L \\
U G / L \\
U G / L \\
U G / L \\
U G / L \\
U G / L \\
U G / L \\
U G / L \\
U G / L \\
U G / L \\
U G / L \\
U G / L \\
U G / L \\
U G / L \\
U G / L \\
U G / L \\
U G / L \\
U G / L \\
U G / L \\
U G / L \\
U G / L \\
U G / L \\
U G / L \\
U G / L \\
U G / L \\
U G / L \\
U G / L \\
U G / L \\
U G / L \\
U G / L \\
U G / L \\
U G / L \\
U G / L \\
U G / L \\
U G / L \\
U G / L \\
U G / L \\
U G / L \\
U G / L \\
U G / L \\
U\end{array}$ & $\begin{array}{l}\text { EPA } 8260 \\
\text { EPA CLP } \\
\text { EPA CLP } \\
\text { EPA CLP } \\
\text { EPA CLP } \\
\text { EPA CLP } \\
\text { EPA CLP } \\
\text { EPA CLP } \\
\text { EPA CLP } \\
\text { EPA CLP } \\
\text { EPA CLP } \\
\text { EPA CLP } \\
\text { EPA CLP } \\
\text { EPA CLP } \\
\text { EPA CLP } \\
\text { EPA CLP } \\
\text { EPA CLP } \\
\text { EPA CLP } \\
\text { EPA CLP } \\
\text { EPA CLP } \\
\text { EPA CLP } \\
\text { EPA CLP } \\
\text { EPA CLP } \\
\text { EPA CLP } \\
\text { EPA CLP } \\
\text { EPA CLP } \\
\text { EPA CLP } \\
\text { EPA CLP } \\
\text { EPA CLP } \\
\text { EPA CLP } \\
\text { EPA CLP } \\
\text { EPA CLP } \\
\text { EPA } 601 \\
\text { EPA CLP } \\
\text { EPA CLP } \\
\text { EPA CLP } \\
\text { EPA CLP } \\
\text { EPA CLP } \\
\text { EPA CLP } \\
\text { EPA CLP } \\
\text { EPA CLP } \\
\text { EPA CLP } \\
\text { EPA CLP } \\
\text { EPA CLP } \\
\text { EPA CLP } \\
\text { EPA CLP } \\
\text { EPA CLP } \\
\text { EPA CLP } \\
\text { EPA CLP } \\
\text { EPA CLP } \\
\text { EPA CLP } \\
\text { EPA CLP } \\
\text { EPA CLP } \\
\text { EPA CLP } \\
\text { EPA CLP } \\
\text { EPA CLP } \\
\text { EPA CLP } \\
\text { EPA CLP }\end{array}$ \\
\hline
\end{tabular}




\begin{tabular}{|c|c|c|c|c|c|c|}
\hline WSSRAP 10 & DATE_SAM & PARAMETER & CONC & DL & UNITS & METHOD \\
\hline $\begin{array}{l}\text { SP-5303-1197-L } \\
\text { SP- } 5303-1197-L \\
\text { SP }-5303-1197-L \\
\text { SP-5303-1197-L } \\
\text { SP-5303-1197-L } \\
\text { SP-5303-1197-L } \\
\text { SP-5303-1197-L } \\
\text { SP-5303-1197-L } \\
\text { SP-5303-1197-L } \\
\text { SP-5303-1197-L } \\
\text { SP-5303-1197-L } \\
\text { SP-5303-1297-L } \\
\text { SP-5303-1297-L } \\
\text { SP-5303-1297-L } \\
\text { SP-5303-1297-L } \\
\text { SP-5303-1297-L } \\
\text { SP-5303-1297-L } \\
\text { SP-5303-1297-L } \\
\text { SP-5303-1297-L } \\
\text { SP-5303-1297-L } \\
\text { SP-5303-1297-L } \\
\text { SP-5303-1297-L } \\
\text { SP-5303-1297-L } \\
\text { SP-5303-1297-L } \\
\text { SP-5303-1297-L } \\
\text { SP-5303-1297-L } \\
\text { SP-5303-1297-L } \\
\text { SP-5303-1297-L } \\
\text { SP-5303-1297-L } \\
\text { SP-5303-1297-L } \\
\text { SP-5303-1297-L } \\
\text { SP-5303-1297-L } \\
\text { SP-5303-1297-L } \\
\text { SP-5303-1297-L } \\
\text { SP-5303-1297-L } \\
\text { SP-5303-1297-L } \\
\text { SP-5303-1297-L } \\
\text { SP-5303-1297-L } \\
\text { SP-5303-1297-L } \\
\text { SP-5303-1297-L } \\
\text { SP-5303-1297-L } \\
\text { SP-5303-1297-L } \\
\text { SP-5303-1297-L } \\
\text { SP-5303-1297-L } \\
\text { SP-5303-1297-L } \\
\text { SP-5304-0198-L } \\
\text { SP-5304-1097-L } \\
\text { SP-5304-1097-L } \\
\text { SP-5304-1097-L } \\
\text { SP-5304-1097-L } \\
\text { SP-5304-1097-L } \\
\text { SP-5304-1097-L } \\
\text { SP-5304-1097-L } \\
\text { SP-5304-1097-L } \\
\text { SP-5304-1097-L } \\
\text { SP-5304-1097-L } \\
\text { SP-5304-1097-L } \\
\text { SP-5304-1097-L } \\
\text { SP-5304-1097-L } \\
\text { SP-5304-1097-L } \\
\text { SP-5304-1097-L }\end{array}$ & $\begin{array}{l}11 / 20 / 97 \\
11 / 20 / 97 \\
11 / 20 / 97 \\
11 / 20 / 97 \\
11 / 20 / 97 \\
11 / 20 / 97 \\
11 / 20 / 97 \\
11 / 20 / 97 \\
11 / 20 / 97 \\
11 / 20 / 97 \\
11 / 20 / 97 \\
12 / 23 / 97 \\
12 / 23 / 97 \\
12 / 23 / 97 \\
12 / 23 / 97 \\
12 / 23 / 97 \\
12 / 23 / 97 \\
12 / 23 / 97 \\
12 / 23 / 97 \\
12 / 23 / 97 \\
12 / 23 / 97 \\
12 / 23 / 97 \\
12 / 23 / 97 \\
12 / 23 / 97 \\
12 / 23 / 97 \\
12 / 23 / 97 \\
12 / 23 / 97 \\
12 / 23 / 97 \\
12 / 23 / 97 \\
12 / 23 / 97 \\
12 / 23 / 97 \\
12 / 23 / 97 \\
12 / 23 / 97 \\
12 / 23 / 97 \\
12 / 23 / 97 \\
12 / 23 / 97 \\
12 / 23 / 97 \\
12 / 23 / 97 \\
12 / 23 / 97 \\
12 / 23 / 97 \\
12 / 23 / 97 \\
12 / 23 / 97 \\
12 / 23 / 97 \\
12 / 23 / 97 \\
12 / 23 / 97 \\
01 / 15 / 98 \\
10 / 29 / 97 \\
10 / 29 / 97 \\
10 / 29 / 97 \\
10 / 29 / 97 \\
10 / 29 / 97 \\
10 / 29 / 97 \\
10 / 29 / 97 \\
10 / 29 / 97 \\
10 / 29 / 97 \\
10 / 29 / 97 \\
10 / 29 / 97 \\
10 / 29 / 97 \\
10 / 29 / 97 \\
10 / 29 / 97 \\
10 / 29 / 97\end{array}$ & 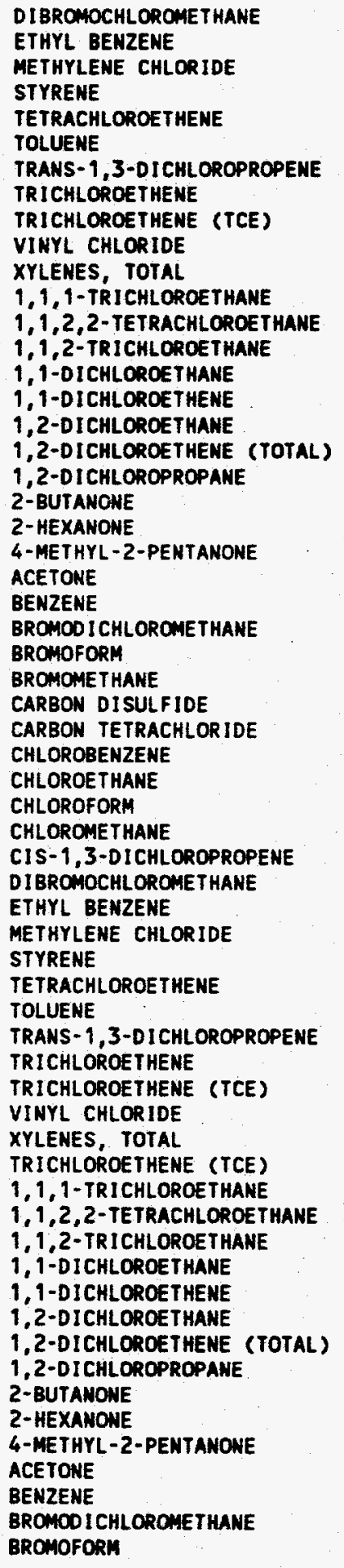 & $\begin{array}{l}\text { ND } \\
\text { ND } \\
\text { ND } \\
\text { ND } \\
\text { ND } \\
\text { ND } \\
\text { ND } \\
\text { ND } \\
\text { ND } \\
\text { ND } \\
\text { ND } \\
\text { ND } \\
\text { ND } \\
\text { ND } \\
\text { ND } \\
\text { ND } \\
\text { ND } \\
\text { ND } \\
\text { ND } \\
\text { ND } \\
\text { ND } \\
\text { ND } \\
\text { ND } \\
\text { ND } \\
\text { ND } \\
\text { ND } \\
\text { ND } \\
\text { ND } \\
\text { ND } \\
\text { ND } \\
\text { ND } \\
\text { ND } \\
\text { ND } \\
\text { ND } \\
\text { ND } \\
\text { ND } \\
\text { ND } \\
\text { ND } \\
\text { ND } \\
\text { ND } \\
\text { ND } \\
\text { ND } \\
\text { ND } \\
\text { ND } \\
\text { ND } \\
\text { ND } \\
\text { ND } \\
\text { ND } \\
\text { ND } \\
\text { ND } \\
\text { ND } \\
\text { ND } \\
\text { ND } \\
\text { ND } \\
\text { ND } \\
\text { ND } \\
\text { ND } \\
\text { ND } \\
\text { ND } \\
\text { ND } \\
\text { ND } \\
\text { ND }\end{array}$ & $\begin{array}{l}10 \\
10 \\
10 \\
10 \\
10 \\
10 \\
10 \\
10 \\
1.0 \\
10 \\
10 \\
10 \\
10 \\
10 \\
10 \\
10 \\
10 \\
10 \\
10 \\
10 \\
10 \\
10 \\
10 \\
10 \\
10 \\
10 \\
10 \\
10 \\
10 \\
10 \\
10 \\
10 \\
10 \\
10 \\
10 \\
10 \\
10 \\
10 \\
10 \\
10 \\
10 \\
10 \\
1.0 \\
10 \\
10 \\
1 \\
10 \\
10 \\
10 \\
10 \\
10 \\
10 \\
10 \\
10 \\
10 \\
10 \\
10 \\
10 \\
10 \\
10 \\
10\end{array}$ & 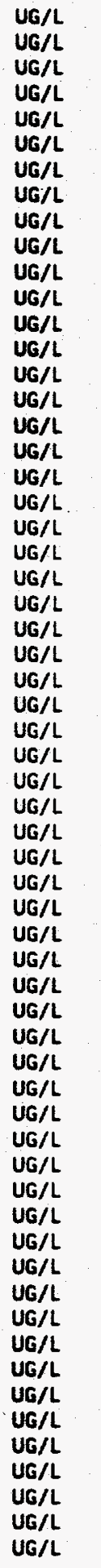 & $\begin{array}{l}\text { EPA CLP } \\
\text { EPA CLP } \\
\text { EPA CLP } \\
\text { EPA CLP } \\
\text { EPA CLP } \\
\text { EPA CLP } \\
\text { EPA CLP } \\
\text { EPA CLP } \\
\text { EPA } 601 \\
\text { EPA CLP } \\
\text { EPA CLP } \\
\text { EPA CLP } \\
\text { EPA CLP } \\
\text { EPA CLP } \\
\text { EPA CLP } \\
\text { EPA CLP } \\
\text { EPA CLP } \\
\text { EPA CLP } \\
\text { EPA CLP } \\
\text { EPA CLP } \\
\text { EPA CLP } \\
\text { EPA CLP } \\
\text { EPA CLP } \\
\text { EPA CLP } \\
\text { EPA CLP } \\
\text { EPA CLP } \\
\text { EPA CLP } \\
\text { EPA CLP } \\
\text { EPA CLP } \\
\text { EPA CLP } \\
\text { EPA CLP } \\
\text { EPA CLP } \\
\text { EPA CLP } \\
\text { EPA CLP } \\
\text { EPA CLP } \\
\text { EPA CLP } \\
\text { EPA CLP } \\
\text { EPA CLP } \\
\text { EPA CLP } \\
\text { EPA CLP } \\
\text { EPA CLP } \\
\text { EPA CLP } \\
\text { EPA } 601 \\
\text { EPA CLP } \\
\text { EPA CLP } \\
\text { EPA B260 } \\
\text { EPA CLP } \\
\text { EPA CLP } \\
\text { EPA CLP } \\
\text { EPA CLP } \\
\text { EPA CLP } \\
\text { EPA CLP } \\
\text { EPA CLP } \\
\text { EPA CLP } \\
\text { EPA CLP } \\
\text { EPA CLP } \\
\text { EPA CLP } \\
\text { EPA CLP } \\
\text { EPA CLP } \\
\text { EPA CLP } \\
\text { EPA CLP }\end{array}$ \\
\hline
\end{tabular}




\begin{tabular}{|c|c|c|c|c|c|c|}
\hline WSSRAP_ID & DATE_SAM & PARAMETER & CONC & DL & UNITS & METHOD \\
\hline 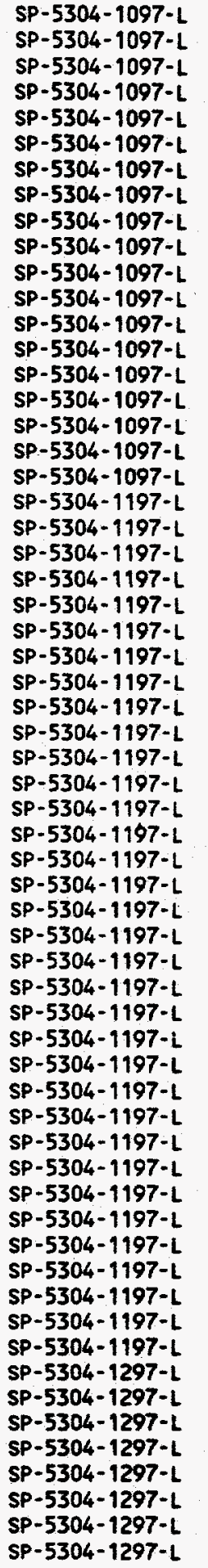 & $\begin{array}{l}10 / 29 / 97 \\
10 / 29 / 97 \\
10 / 29 / 97 \\
10 / 29 / 97 \\
10 / 29 / 97 \\
10 / 29 / 97 \\
10 / 29 / 97 \\
10 / 29 / 97 \\
10 / 29 / 97 \\
10 / 29 / 97 \\
10 / 29 / 97 \\
10 / 29 / 97 \\
10 / 29 / 97 \\
10 / 29 / 97 \\
10 / 29 / 97 \\
10 / 29 / 97 \\
10 / 29 / 97 \\
10 / 29 / 97 \\
10 / 29 / 97 \\
111 / 20 / 97 \\
11 / 20 / 97 \\
11 / 20 / 97 \\
11 / 20 / 97 \\
11 / 20 / 97 \\
11 / 20 / 97 \\
11 / 20 / 97 \\
11 / 20 / 97 \\
11 / 20 / 97 \\
111 / 20 / 97 \\
11 / 20 / 97 \\
11 / 20 / 97 \\
11 / 20 / 97 \\
11 / 20 / 97 \\
11 / 20 / 97 \\
11 / 20 / 97 \\
11 / 20 / 97 \\
11 / 20 / 97 \\
11 / 20 / 97 \\
11120 / 97 \\
11 / 20 / 97 \\
11 / 20 / 97 \\
11 / 20 / 97 \\
11 / 20 / 97 \\
11 / 20 / 97 \\
11 / 20 / 97 \\
11 / 20 / 97 \\
11 / 20 / 97 \\
11 / 20 / 97 \\
11 / 20 / 97 \\
11 / 20 / 97 \\
11 / 20 / 97 \\
11 / 20 / 97 \\
11120 / 97 \\
12 / 23 / 97 \\
12 / 23 / 97 \\
12 / 23 / 97 \\
12 / 23 / 97 \\
12 / 23 / 97 \\
12 / 23 / 97 \\
12 / 23 / 97 \\
12 / 23 / 97\end{array}$ & 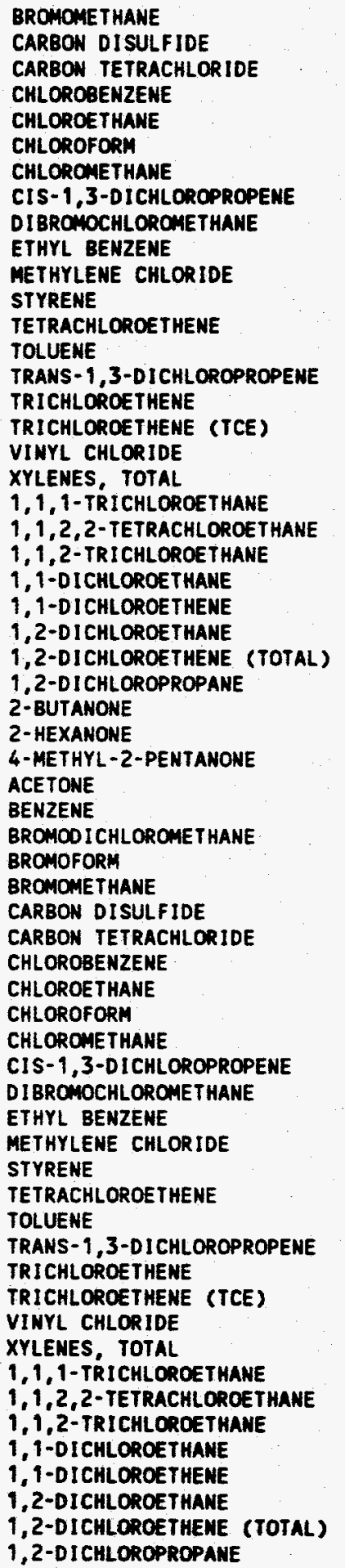 & $\begin{array}{l}\text { ND } \\
\text { ND } \\
\text { ND } \\
\text { ND } \\
\text { ND } \\
\text { ND } \\
\text { ND } \\
\text { ND } \\
\text { ND } \\
\text { ND } \\
\text { ND } \\
\text { ND } \\
\text { ND } \\
\text { ND } \\
\text { ND } \\
\text { ND } \\
\text { ND } \\
\text { ND } \\
\text { ND } \\
\text { ND } \\
\text { ND } \\
\text { ND } \\
\text { ND } \\
\text { ND } \\
\text { ND } \\
\text { ND } \\
\text { ND } \\
\text { ND } \\
\text { ND } \\
\text { ND } \\
\text { ND } \\
\text { ND } \\
\text { ND } \\
\text { ND } \\
\text { ND } \\
\text { ND } \\
\text { ND } \\
\text { ND } \\
\text { ND } \\
\text { ND } \\
\text { ND } \\
\text { ND } \\
\text { ND } \\
\text { ND } \\
\text { ND } \\
\text { ND } \\
\text { ND } \\
\text { ND } \\
\text { ND } \\
\text { ND } \\
\text { ND } \\
\text { ND } \\
\text { ND } \\
\text { ND } \\
\text { ND } \\
\text { ND } \\
\text { ND } \\
\text { ND } \\
\text { ND } \\
\text { ND } \\
\text { ND }\end{array}$ & $\begin{array}{l}10 \\
10 \\
10 \\
10 \\
10 \\
10 \\
10 \\
10 \\
10 \\
10 \\
10 \\
10 \\
10 \\
10 \\
10 \\
10 \\
1.0 \\
10 \\
10 \\
10 \\
10 \\
10 \\
10 \\
10 \\
10 \\
10 \\
10 \\
10 \\
10 \\
10 \\
10 \\
10 \\
10 \\
10 \\
10 \\
10 \\
10 \\
10 \\
10 \\
10 \\
10 \\
10 \\
10 \\
10 \\
10 \\
10 \\
10 \\
10 \\
10 \\
10 \\
1.0 \\
10 \\
10 \\
10 \\
10 \\
10 \\
10 \\
10 \\
10 \\
10 \\
10 \\
10\end{array}$ & 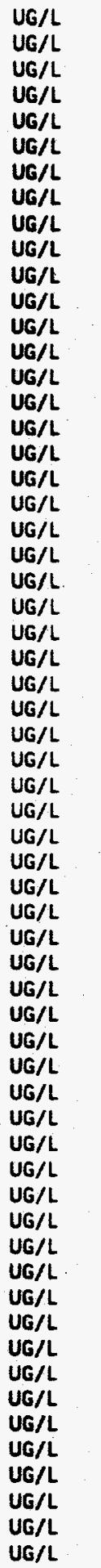 & 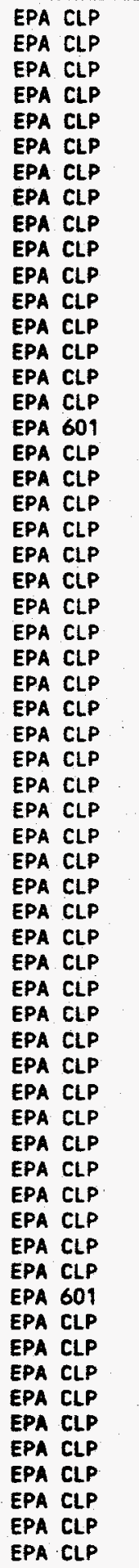 \\
\hline
\end{tabular}




\begin{tabular}{|c|c|c|c|c|c|c|}
\hline WSSRAP_ID & DATE_SAM & PARAMETER & $\operatorname{conc}$ & DL & UNITS & METHOO \\
\hline 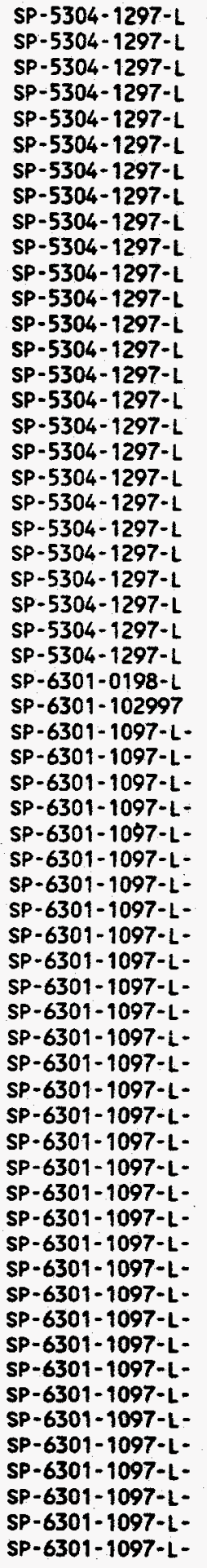 & $\begin{array}{l}12 / 23 / 97 \\
12 / 23 / 97 \\
12 / 23 / 97 \\
12 / 23 / 97 \\
12 / 23 / 97 \\
12 / 23 / 97 \\
12 / 23 / 97 \\
12 / 23 / 97 \\
12 / 23 / 97 \\
12 / 23 / 97 \\
12 / 23 / 97 \\
12 / 23 / 97 \\
12 / 23 / 97 \\
12 / 23 / 97 \\
12 / 23 / 97 \\
12 / 23 / 97 \\
12 / 23 / 97 \\
12 / 23 / 97 \\
12 / 23 / 97 \\
12 / 23 / 97 \\
12 / 23 / 97 \\
12 / 23 / 97 \\
12 / 23 / 97 \\
12 / 23 / 97 \\
12 / 23 / 97 \\
12 / 23 / 97 \\
01 / 15 / 98 \\
10 / 29 / 97 \\
10 / 15 / 97 \\
10 / 15 / 97 \\
10 / 15 / 97 \\
10 / 15 / 97 \\
10 / 15 / 97 \\
10 / 15 / 97 \\
10 / 15 / 97 \\
10 / 15 / 97 \\
10 / 15 / 97 \\
10 / 15 / 97 \\
10 / 15 / 97 \\
10 / 15 / 97 \\
10 / 15 / 97 \\
10 / 15 / 97 \\
10 / 15 / 97 \\
10 / 15 / 97 \\
10 / 15 / 97 \\
10 / 15 / 97 \\
10 / 15 / 97 \\
10 / 15 / 97 \\
10 / 15 / 97 \\
10 / 15 / 97 \\
10 / 15 / 97 \\
10 / 15 / 97 \\
10 / 15 / 97 \\
10 / 15 / 97 \\
10 / 15 / 97 \\
10 / 15 / 97 \\
10 / 15 / 97 \\
10 / 15 / 97 \\
10 / 15 / 97 \\
10 / 15 / 97 \\
10 / 15 / 97\end{array}$ & 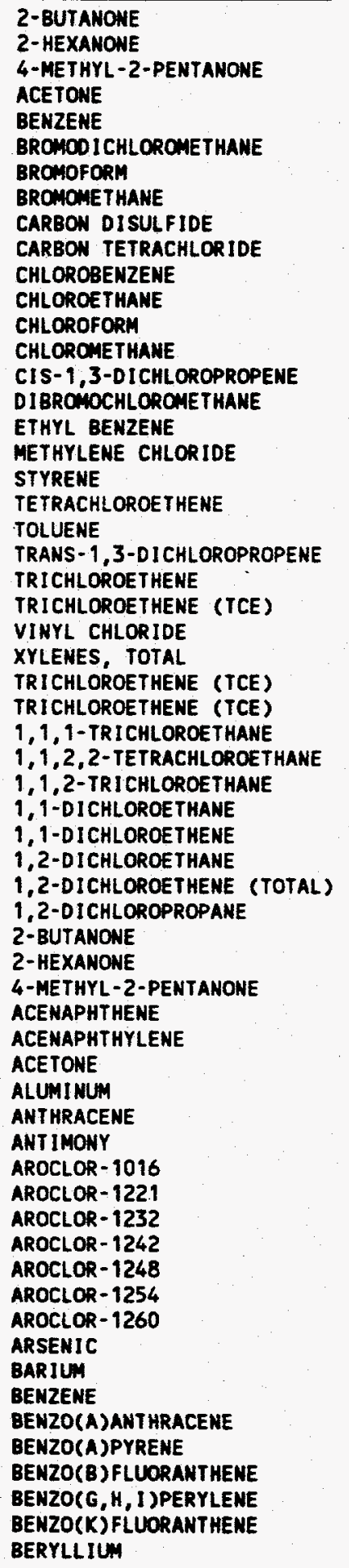 & $\begin{array}{l}\text { ND } \\
\text { ND } \\
\text { ND } \\
\text { ND } \\
\text { ND } \\
\text { ND } \\
\text { ND } \\
\text { ND } \\
\text { ND } \\
\text { ND } \\
\text { ND } \\
\text { ND } \\
\text { ND } \\
\text { ND } \\
\text { ND } \\
\text { ND } \\
\text { ND } \\
\text { ND } \\
\text { ND } \\
\text { ND } \\
\text { ND } \\
\text { ND } \\
\text { ND } \\
\text { ND } \\
\text { ND } \\
\text { ND } \\
\text { ND } \\
\text { ND } \\
\text { ND } \\
\text { ND } \\
\text { ND } \\
\text { ND } \\
\text { ND } \\
\text { ND } \\
\text { ND } \\
\text { ND } \\
\text { ND } \\
\text { ND } \\
\text { ND } \\
\text { ND } \\
\text { ND } \\
\text { ND } \\
\text { I34 } \\
\text { ND } \\
\text { ND } \\
\text { ND } \\
\text { ND } \\
\text { ND } \\
\text { ND } \\
\text { ND } \\
\text { ND } \\
\text { ND } \\
\text { ND } \\
\text { I37 } \\
\text { ND } \\
\text { ND } \\
\text { ND } \\
\text { ND } \\
\text { ND } \\
\text { ND } \\
\text { ND }\end{array}$ & $\begin{array}{l}10 \\
10 \\
10 \\
10 \\
10 \\
10 \\
10 \\
10 \\
10 \\
10 \\
10 \\
10 \\
10 \\
10 \\
10 \\
10 \\
10 \\
10 \\
10 \\
10 \\
10 \\
10 \\
10 \\
1.0 \\
10 \\
10 \\
1 \\
1.0 \\
10 \\
10 \\
10 \\
10 \\
10 \\
10 \\
10 \\
10 \\
10 \\
10 \\
10 \\
0.5 \\
0.5 \\
10 \\
60.0 \\
0.5 \\
11.0 \\
1.0 \\
1.0 \\
1.0 \\
1.0 \\
1.0 \\
1.0 \\
1.0 \\
4.0 \\
74.0 \\
10 \\
0.5 \\
0.5 \\
0.5 \\
0.5 \\
0.5 \\
1.0\end{array}$ & 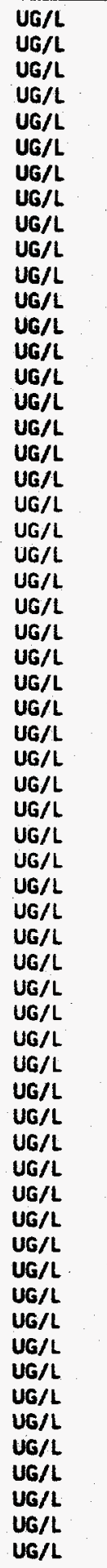 & $\begin{array}{l}\text { EPA CLP } \\
\text { EPA CLP } \\
\text { EPA CLP } \\
\text { EPA CLP } \\
\text { EPA CLP } \\
\text { EPA CLP } \\
\text { EPA CLP } \\
\text { EPA CLP } \\
\text { EPA CLP } \\
\text { EPA CLP } \\
\text { EPA CLP } \\
\text { EPA CLP } \\
\text { EPA CLP } \\
\text { EPA CLP } \\
\text { EPA } C L P \\
\text { EPA CLP } \\
\text { EPA CLP } \\
\text { EPA CLP } \\
\text { EPA CLP } \\
\text { EPA CLP } \\
\text { EPA CLP } \\
\text { EPA CLP } \\
\text { EPA CLP } \\
\text { EPA } 601 \\
\text { EPA } C L P \\
\text { EPA CLP } \\
\text { EPA } 8260 \\
\text { EPA } 601 \\
\text { EPA CLP } \\
\text { EPA CLP } \\
\text { EPA CLP } \\
\text { EPA CLP } \\
\text { EPA CLP } \\
\text { EPA CLP } \\
\text { EPA CLP } \\
\text { EPA CLP } \\
\text { EPA CLP } \\
\text { EPA CLP } \\
\text { EPA CLP } \\
\text { EPA } 8310 \\
\text { EPA } 8310 \\
\text { EPA CLP } \\
\text { EPA } 6010 A \\
\text { EPA } 8310 \\
\text { EPA } 6010 A \\
\text { EPA } 8081 \\
\text { EPA } 8081 \\
\text { EPA } 8081 \\
\text { EPA } 8081 \\
\text { EPA } 8081 \\
\text { EPA } 8081 \\
\text { EPA } 8081 \\
\text { EPA } 6010 A \\
\text { EPA } 6010 A \\
\text { EPA CLP } \\
\text { EPA } 8310 \\
\text { EPA } 8310 \\
\text { EPA } 8310 \\
\text { EPA } 8310 \\
\text { EPA } 8310 \\
\text { EPA } 6010 A\end{array}$ \\
\hline
\end{tabular}




\begin{tabular}{|c|c|c|c|c|c|c|}
\hline WSSRAP_ID & DATE_SAM & PARAMETER & conc & $D L$ & UNITS & METHOD \\
\hline $\begin{array}{l}\text { SP-6301-1097-L- } \\
\text { SP-6301-1097-L- } \\
\text { SP-6301-1097-L- } \\
\text { SP-6301-1097-L- } \\
\text { SP-6301-1097-L- } \\
\text { SP-6301-1097-L- } \\
\text { SP-6301-1097-L- } \\
\text { SP-6301-1097-L- } \\
\text { SP-6301-1097-L- } \\
\text { SP-6301-1097-L- } \\
\text { SP-6301-1097-L- } \\
\text { SP-6301-1097-L- } \\
\text { SP-6301-1097-L- } \\
\text { SP-6301-1097-L- } \\
\text { SP-6301-1097-L- } \\
\text { SP-6301-1097-L- } \\
\text { SP-6301-1097-L- } \\
\text { SP-6301-1097-L- } \\
\text { SP-6301-1097-L- } \\
\text { SP-6301-1097-L- } \\
\text { SP-6301-1097-L- } \\
\text { SP-6301-1097-L- } \\
\text { SP-6301-1097-L- } \\
\text { SP-6301-1097-L- } \\
\text { SP-6301-1097-LL- } \\
\text { SP-6301-1097-L- } \\
\text { SP-6301-1097-L- } \\
\text { SP-6301-1097-L- } \\
\text { SP-6301-1097-L- } \\
\text { SP-6301-1097-L- } \\
\text { SP-6301-1097-L- } \\
\text { SP-6301-1097-L- } \\
\text { SP-6301-1097-L- } \\
\text { SP-6301-1097-L- } \\
\text { SP-6301-1097-L- } \\
\text { SP-6301-1097-L- } \\
\text { SP-6301-1097-L- } \\
\text { SP-6301-1097-L- } \\
\text { SP-6301-1097-L- } \\
\text { SP-6301-1097-L- } \\
\text { SP-6301-1097-L- } \\
\text { SP-6301-1097-L- } \\
\text { SP-6301-1097-L- } \\
\text { SP-6301-1097-L- } \\
\text { SP-6301-1097-L- } \\
\text { SP-6301-1097-L- } \\
\text { SP-6301-1097-L- } \\
\text { SP-6301-1097-L- } \\
\text { SP-6301-1097-L- } \\
\text { SP-6301-1097-L- } \\
\text { SP-6301-1097-L- } \\
\text { SP-6301-1097-L- } \\
\text { SP-6301-1097-L- } \\
\text { SP-6301-1097-L- } \\
\text { SP-6301-1097-L- } \\
\text { SP-6301-1097-L- } \\
\text { SP-6301-1097-L- } \\
\text { SP-6301-1097-L- } \\
\text { SP-6301-1097-L- } \\
\text { SP-6301-1097-L- } \\
\text { SP-6301-1097-L- }\end{array}$ & $\begin{array}{l}10 / 15 / 97 \\
10 / 15 / 97 \\
10 / 15 / 97 \\
10 / 15 / 97 \\
10 / 15 / 97 \\
10 / 15 / 97 \\
10 / 15 / 97 \\
10 / 15 / 97 \\
10 / 15 / 97 \\
10 / 15 / 97 \\
10 / 15 / 97 \\
10 / 15 / 97 \\
10 / 15 / 97 \\
10 / 15 / 97 \\
10 / 15 / 97 \\
10 / 15 / 97 \\
10 / 15 / 97 \\
10 / 15 / 97 \\
10 / 15 / 97 \\
10 / 15 / 97 \\
10 / 15 / 97 \\
10 / 15 / 97 \\
10 / 15 / 97 \\
10 / 15 / 97 \\
10 / 15 / 97 \\
10 / 15 / 97 \\
10 / 15 / 97 \\
10 / 15 / 97 \\
10 / 15 / 97 \\
10 / 15 / 97 \\
10 / 15 / 97 \\
10 / 15 / 97 \\
10 / 15 / 97 \\
10 / 15 / 97 \\
10 / 15 / 97 \\
10 / 15 / 97 \\
10 / 15 / 97 \\
10 / 15 / 97 \\
10 / 15 / 97 \\
10 / 15 / 97 \\
10 / 15 / 97 \\
10 / 15 / 97 \\
10 / 15 / 97 \\
10 / 15 / 97 \\
10 / 15 / 97 \\
10 / 15 / 97 \\
10 / 15 / 97 \\
10 / 15 / 97 \\
10 / 15 / 97 \\
10 / 15 / 97 \\
10 / 15 / 97 \\
10 / 15 / 97 \\
10 / 15 / 97 \\
10 / 15 / 97 \\
10 / 15 / 97 \\
10 / 15 / 97 \\
10 / 15 / 97 \\
10 / 15 / 97 \\
10 / 15 / 97 \\
10 / 15 / 97 \\
10 / 15 / 97\end{array}$ & 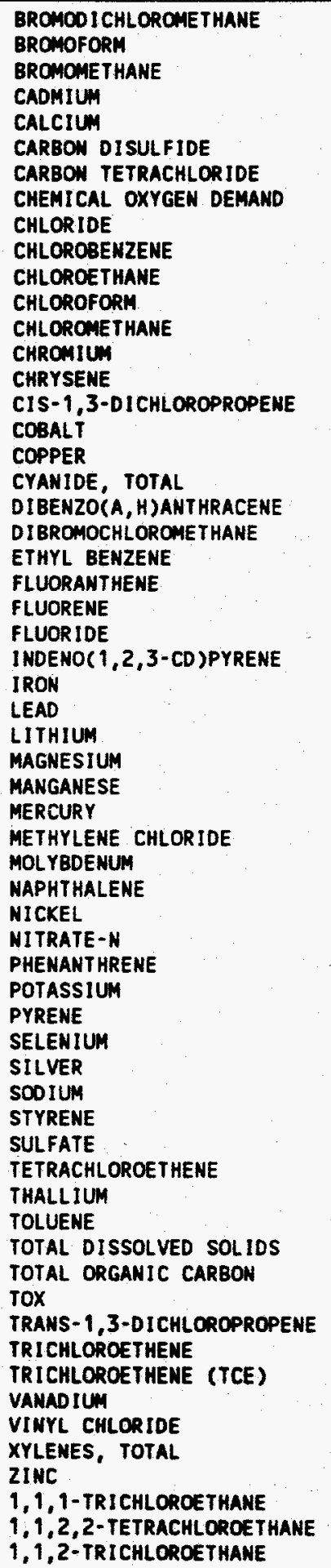 & 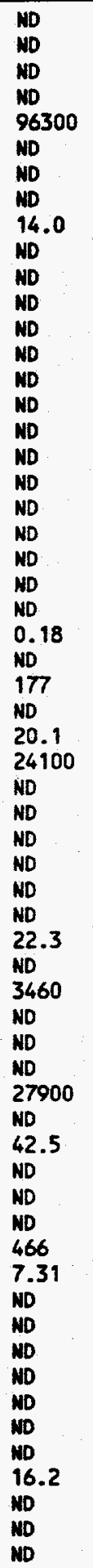 & 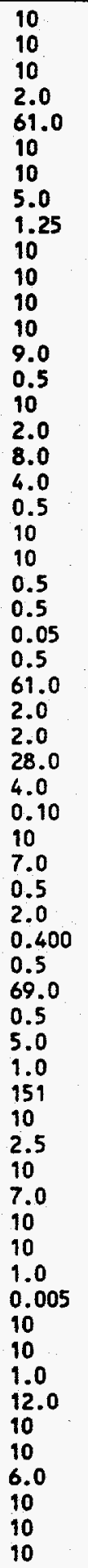 & $\begin{array}{l}\text { UG/L } \\
U G / L \\
U G / L \\
U G / L \\
U G / L \\
U G / L \\
U G / L \\
U G / L \\
\text { MG/L } \\
U G / L \\
U G / L \\
U G / L \\
U G / L \\
U G / L \\
U G / L \\
U G / L \\
U G / L \\
U G / L \\
U G / L \\
U G / L \\
U G / L \\
U G / L \\
U G / L \\
U G / L \\
M G / L \\
U G / L \\
U G / L \\
U G / L \\
U G / L \\
U G / L \\
U G / L \\
U G / L \\
U G / L \\
U G / L \\
U G / L \\
U G / L \\
M G / L \\
U G / L \\
U G / L \\
U G / L \\
U G / L \\
U G / L \\
U G / L \\
U G / L \\
M G / L \\
U G / L \\
U G / L \\
U G / L \\
M G / L \\
\text { UG/L} \\
M G / L \\
U G / L \\
U G / L \\
U G / L \\
U G / L \\
U G / L \\
U G / L \\
U G / L \\
U G / L \\
U G / L \\
U G / L \\
U\end{array}$ & 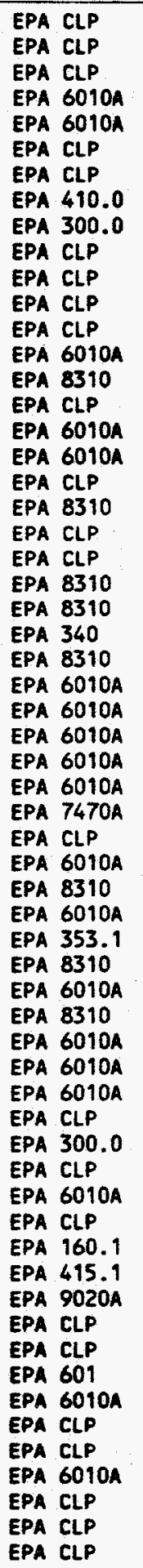 \\
\hline
\end{tabular}




\begin{tabular}{|c|c|c|c|c|c|c|c|}
\hline USSRAP_ID & DATE_SAM & PARAMETER & conc & $D L$ & UNITS & METHOD & \\
\hline $\begin{array}{l}\text { SP-6301-1097-L- } \\
\text { SP-6301-1097-L- } \\
\text { SP-6301-1097-L- } \\
\text { SP-6301-1097-L- } \\
\text { SP-6301-1097-L- } \\
\text { SP-6301-1097-L- } \\
\text { SP-6301-1097-L- } \\
\text { SP-6301-1097-L- } \\
\text { SP-6301-1097-L- } \\
\text { SP-6301-1097-L- } \\
\text { SP-6301-1097-L- } \\
\text { SP-6301-1097-L- } \\
\text { SP-6301-1097-L- } \\
\text { SP-6301-1097-L- } \\
\text { SP-6301-1097-L- } \\
\text { SP-6301-1097-L- } \\
\text { SP-6301-1097-L- } \\
\text { SP-6301-1097-L- } \\
\text { SP-6301-1097-L- } \\
\text { SP-6301-1097-L- } \\
\text { SP-6301-1097-L- } \\
\text { SP-6301-1097-L- } \\
\text { SP-6301-1097-L- } \\
\text { SP-6301-1097-L- } \\
\text { SP-6301-1097-L- } \\
\text { SP-6301-1097-L- } \\
\text { SP-6301-1097-L- } \\
\text { SP-6301-1097-L- } \\
\text { SP-6301-1097-L- } \\
\text { SP-6301-1097-L- } \\
\text { SP-6301-1097-L- } \\
\text { SP-6301-1097-L- } \\
\text { SP-6301-1097-L- } \\
\text { SP-6301-1097-L- } \\
\text { SP-6301-1097-L- } \\
\text { SP-6301-1097-L- } \\
\text { SP-6301-1097-L- } \\
\text { SP-6301-1097-L- } \\
\text { SP-6301-1097-L- } \\
\text { SP-6301-1097-L- } \\
\text { SP-6301-1097-L- } \\
\text { SP-6301-1097-L- } \\
\text { SP-6301-1097-L- } \\
\text { SP-6301-1097-L- } \\
\text { SP-6301-1097-L- } \\
\text { SP-6301-1097-L- } \\
\text { SP-6301-1097-L- } \\
\text { SP-6301-1097-L- } \\
\text { SP-6301-1097-L- } \\
\text { SP-6301-1097-L- } \\
\text { SP-6301-1097-L- } \\
\text { SP-6301-1097-L- } \\
\text { SPP-6301-1097-L- } \\
\text { SP-6301-1097-LL- } \\
\text { SP-6301-1097-L- } \\
\text { SP-6301-1097-L- } \\
\text { SP-6301-1097-L- } \\
\text { SP-6301-1097-L- } \\
\text { SP-6301-1097-L- } \\
\text { SP-6301-1097-L- } \\
\text { SP-6301-1097-L- }\end{array}$ & $\begin{array}{l}10 / 15 / 97 \\
10 / 15 / 97 \\
10 / 15 / 97 \\
10 / 15 / 97 \\
10 / 15 / 97 \\
10 / 15 / 97 \\
10 / 15 / 97 \\
10 / 15 / 97 \\
10 / 15 / 97 \\
10 / 15 / 97 \\
10 / 15 / 97 \\
10 / 15 / 97 \\
10 / 15 / 97 \\
10 / 15 / 97 \\
10 / 15 / 97 \\
10 / 15 / 97 \\
10 / 15 / 97 \\
10 / 15 / 97 \\
10 / 15 / 97 \\
10 / 15 / 97 \\
10 / 15 / 97 \\
10 / 15 / 97 \\
10 / 15 / 97 \\
10 / 15 / 97 \\
10 / 15 / 97 \\
10 / 15 / 97 \\
10 / 15 / 97 \\
10 / 15 / 97 \\
10 / 15 / 97 \\
10 / 15 / 97 \\
10 / 15 / 97 \\
10 / 15 / 97 \\
10 / 15 / 97 \\
10 / 15 / 97 \\
10 / 15 / 97 \\
10 / 15 / 97 \\
10 / 15 / 97 \\
10 / 15 / 97 \\
10 / 15 / 97 \\
10 / 15 / 97 \\
10 / 15 / 97 \\
10 / 15 / 97 \\
10 / 15 / 97 \\
10 / 15 / 97 \\
10 / 15 / 97 \\
10 / 15 / 97 \\
10 / 15 / 97 \\
10 / 15 / 97 \\
10 / 15 / 97 \\
10 / 15 / 97 \\
10 / 15 / 97 \\
10 / 15 / 97 \\
10 / 15 / 97 \\
10 / 15 / 97 \\
10 / 15 / 97 \\
10 / 15 / 97 \\
10 / 15 / 97 \\
10 / 15 / 97 \\
10 / 15 / 97 \\
10 / 15 / 97 \\
10 / 15 / 97\end{array}$ & 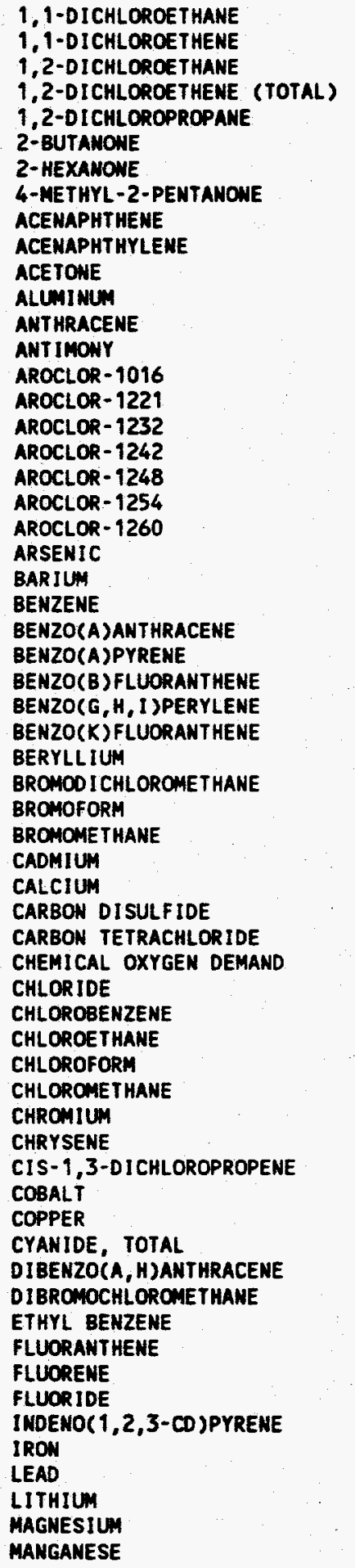 & 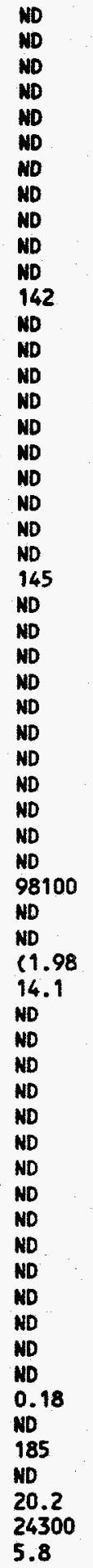 & $\begin{array}{l}10 \\
10 \\
10 \\
10 \\
10 \\
10 \\
10 \\
10 \\
0.5 \\
0.5 \\
10 \\
60.0 \\
0.5 \\
11.0 \\
1.0 \\
1.0 \\
1.0 \\
1.0 \\
1.0 \\
1.0 \\
1.0 \\
4.0 \\
74.0 \\
10 \\
0.5 \\
0.5 \\
0.5 \\
0.5 \\
0.5 \\
1.0 \\
10 \\
10 \\
10 \\
2.0 \\
61.0 \\
10 \\
10 \\
5.0 \\
1.25 \\
10 \\
10 \\
10 \\
10 \\
9.0 \\
0.5 \\
10 \\
2.0 \\
8.0 \\
4.0 \\
0.5 \\
10 \\
10 \\
0.5 \\
0.5 \\
0.05 \\
0.5 \\
61.0 \\
2.0 \\
2.0 \\
28.0 \\
4.0\end{array}$ & 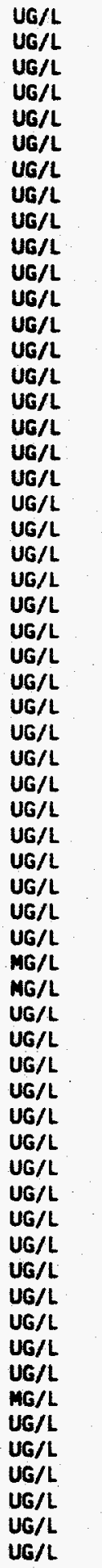 & 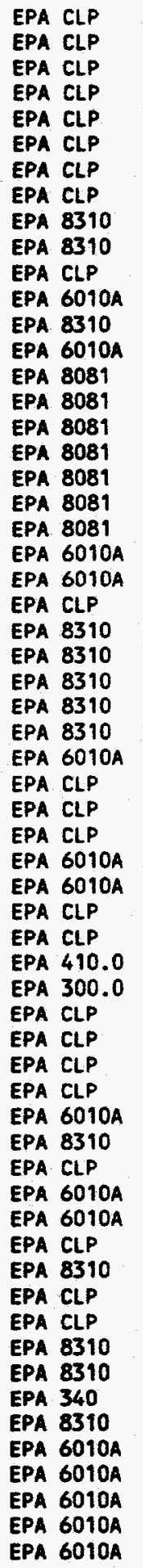 & - \\
\hline
\end{tabular}




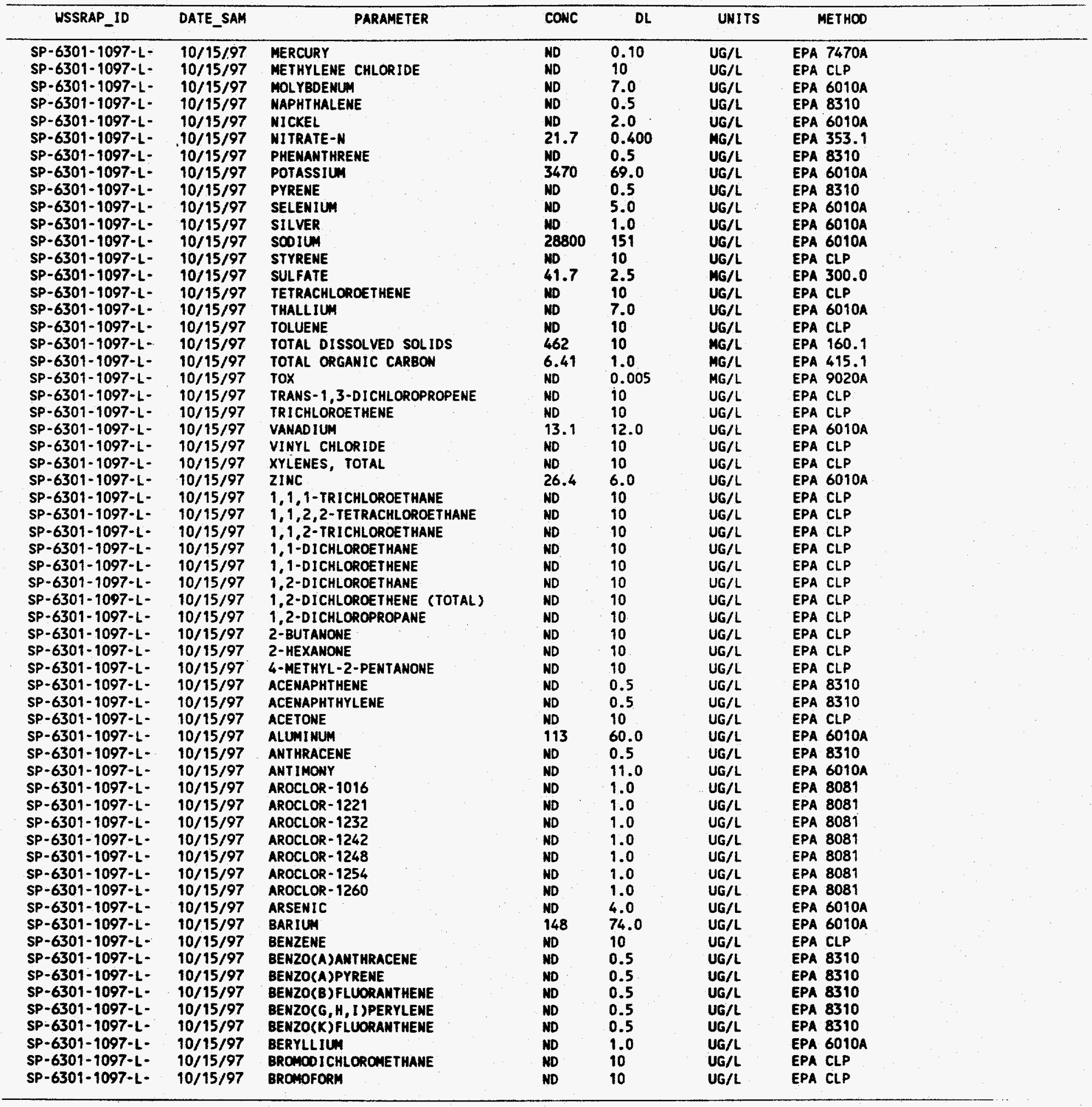




\begin{tabular}{|c|c|c|c|c|c|c|}
\hline USSRAP_ID & DATE_SAM & PARAMETER & CONC & DL & UNITS & METHOD \\
\hline $\begin{array}{l}\text { SP-6301-1097-L- } \\
\text { SP-6301-1097-L- } \\
\text { SP-6301-1097-L- } \\
\text { SP-6301-1097-L- } \\
\text { SP-6301-1097-L- } \\
\text { SP-6301-1097-L- } \\
\text { SP-6301-1097-L- } \\
\text { SP-6301-1097-L- } \\
\text { SP-6301-1097-L- } \\
\text { SP-6301-1097-L- } \\
\text { SP-6301-1097-L- } \\
\text { SP-6301-1097-L- } \\
\text { SP-6301-1097-L- } \\
\text { SP-6301-1097-L- } \\
\text { SP-6301-1097-L- } \\
\text { SP-6301-1097-L- } \\
\text { SP-6301-1097-L- } \\
\text { SP-6301-1097-L- } \\
\text { SP-6301-1097-L- } \\
\text { SP-6301-1097-L- } \\
\text { SP-6301-1097-L- } \\
\text { SP-6301-1097-L- } \\
\text { SP-6301-1097-L- } \\
\text { SP-6301-1097-L- } \\
\text { SP-6301-1097-L- } \\
\text { SP-6301-1097-L- } \\
\text { SP-6301-1097-L- } \\
\text { SP-6301-1097-L- } \\
\text { SP-6301-1097-L- } \\
\text { SP-6301-1097-L- } \\
\text { SP-6301-1097-L- } \\
\text { SP-6301-1097-L- } \\
\text { SP-6301-1097-L- } \\
\text { SP-6301-1097-L- } \\
\text { SP-6301-1097-L- } \\
\text { SP-6301-1097-L- } \\
\text { SP-6301-1097-L- } \\
\text { SP-6301-1097-L- } \\
\text { SP-6301-1097-L- } \\
\text { SP-6301-1097-L- } \\
\text { SP-6301-1097-L- } \\
\text { SP-6301-1097-L- } \\
\text { SP-6301-1097-L- } \\
\text { SP-6301-1097-L- } \\
\text { SP-6301-1097-L- } \\
\text { SP-6301-1097-L- } \\
\text { SP-6301-1097-L- } \\
\text { SP-6301-1097-L- } \\
\text { SPP-6301-1097-L- } \\
\text { SP-6301-1097-L- } \\
\text { SP-6301-1097-L- } \\
\text { SP-6301-1097-L- } \\
\text { SP-6301-1097-L- } \\
\text { SP-6301-1097-L- } \\
\text { SP-6301-1097-L- } \\
\text { SP-6301-1097-L- } \\
\text { SP-6301-1097-L- } \\
\text { SP-6301-1097-L- } \\
\text { SP }-6301-1097-L- \\
\text { SP-6301-1097-L- } \\
\text { SP-6301-1097-L- }\end{array}$ & $\begin{array}{l}10 / 15 / 97 \\
10 / 15 / 97 \\
10 / 15 / 97 \\
10 / 15 / 97 \\
10 / 15 / 97 \\
10 / 15 / 97 \\
10 / 15 / 97 \\
10 / 15 / 97 \\
10 / 15 / 97 \\
10 / 15 / 97 \\
10 / 15 / 97 \\
10 / 15 / 97 \\
10 / 15 / 97 \\
10 / 15 / 97 \\
10 / 15 / 97 \\
10 / 15 / 97 \\
10 / 15 / 97 \\
10 / 15 / 97 \\
10 / 15 / 97 \\
10 / 15 / 97 \\
10 / 15 / 97 \\
10 / 15 / 97 \\
10 / 15 / 97 \\
10 / 15 / 97 \\
10 / 15 / 97 \\
10 / 15 / 97 \\
10 / 15 / 97 \\
10 / 15 / 97 \\
10 / 15 / 97 \\
10 / 15 / 97 \\
10 / 15 / 97 \\
10 / 15 / 97 \\
10 / 15 / 97 \\
10 / 15 / 97 \\
10 / 15 / 97 \\
10 / 15 / 97 \\
10 / 15 / 97 \\
10 / 15 / 97 \\
10 / 15 / 97 \\
10 / 15 / 97 \\
10 / 15 / 97 \\
10 / 15 / 97 \\
10 / 15 / 97 \\
10 / 15 / 97 \\
10 / 15 / 97 \\
10 / 15 / 97 \\
10 / 15 / 97 \\
10 / 15 / 97 \\
10 / 15 / 97 \\
10 / 15 / 97 \\
10 / 15 / 97 \\
10 / 15 / 97 \\
10 / 15 / 97 \\
10 / 15 / 97 \\
10 / 15 / 97 \\
10 / 15 / 97 \\
10 / 15 / 97 \\
10 / 15 / 97 \\
10 / 15 / 97 \\
10 / 15 / 97 \\
10 / 15 / 97\end{array}$ & 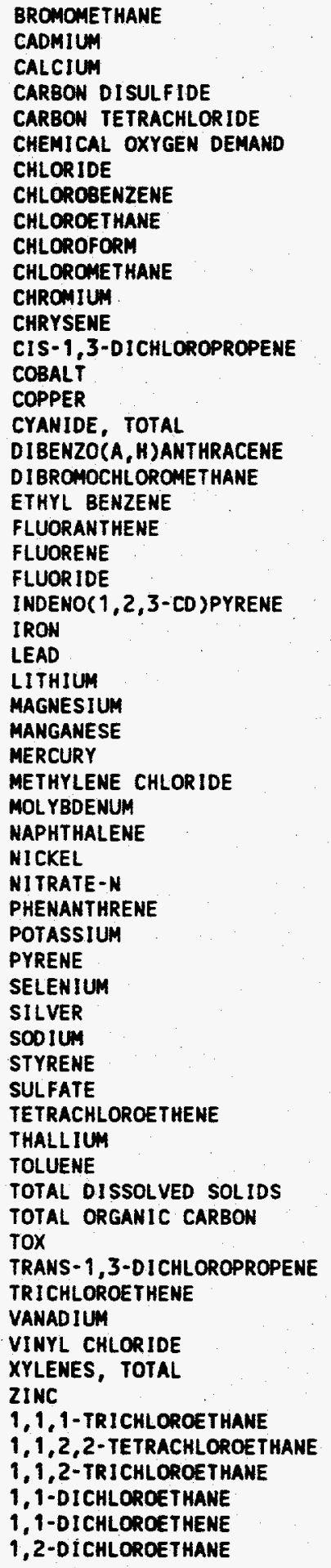 & $\begin{array}{l}\text { ND } \\
\text { ND } \\
10000 \\
\text { ND } \\
\text { ND } \\
\text { ND } \\
\text { 15.2 } \\
\text { ND } \\
\text { ND } \\
\text { ND } \\
\text { ND } \\
\text { ND } \\
\text { ND } \\
\text { ND } \\
\text { ND } \\
\text { ND } \\
\text { ND } \\
\text { ND } \\
\text { ND } \\
\text { ND } \\
\text { ND } \\
\text { ND } \\
\text { O.19 } \\
\text { ND } \\
215 \\
2.0 \\
20.7 \\
24800 \\
\text { ND } \\
\text { ND } \\
\text { ND } \\
\text { ND } \\
\text { ND } \\
\text { ND } \\
22.5 \\
\text { ND } \\
3620 \\
\text { ND } \\
\text { ND } \\
\text { ND } \\
29200 \\
\text { ND } \\
41.4 \\
\text { ND } \\
\text { ND } \\
\text { ND } \\
440 \\
6.22 \\
\text { ND } \\
\text { ND } \\
\text { ND } \\
\text { ND } \\
\text { ND } \\
\text { ND } \\
14.7 \\
\text { ND } \\
\text { ND } \\
\text { ND } \\
\text { ND } \\
\text { ND } \\
\text { ND } \\
\text { D }\end{array}$ & $\begin{array}{l}10 \\
2.0 \\
61.0 \\
10 \\
10 \\
5.0 \\
1.25 \\
10 \\
10 \\
10 \\
10 \\
9.0 \\
0.5 \\
10 \\
2.0 \\
8.0 \\
4.0 \\
0.5 \\
10 \\
10 \\
0.5 \\
0.5 \\
0.05 \\
0.5 \\
61.0 \\
2.0 \\
2.0 \\
28.0 \\
4.0 \\
0.10 \\
10 \\
7.0 \\
0.5 \\
2.0 \\
0.400 \\
0.5 \\
69.0 \\
0.5 \\
5.0 \\
1.0 \\
151 \\
10 \\
2.5 \\
10 \\
7.0 \\
10 \\
10 \\
1.0 \\
0.005 \\
10 \\
10 \\
12.0 \\
10 \\
10 \\
6.0 \\
10 \\
10 \\
10 \\
10 \\
10 \\
10 \\
10 \\
0\end{array}$ & 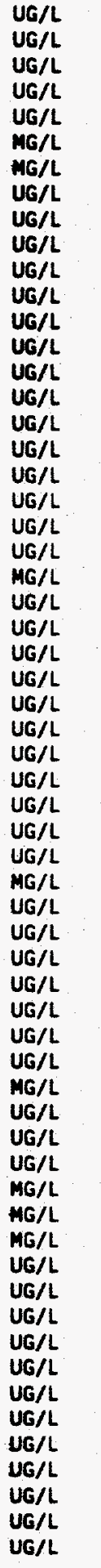 & 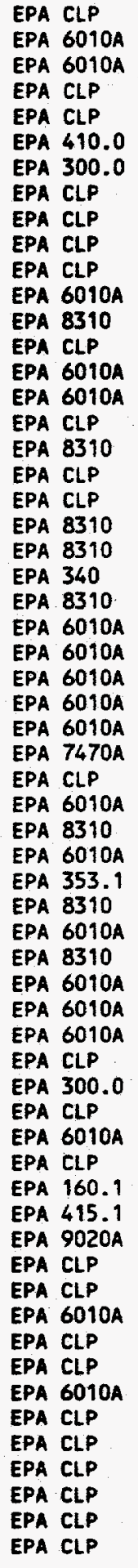 \\
\hline
\end{tabular}




\begin{tabular}{|c|c|c|c|c|c|c|}
\hline USSRAP_ID & DATE_SAM & PARAMETER & CONC & DL & UNITS & METHOO \\
\hline $\begin{array}{l}\text { SP-6301-1097-L- } \\
\text { SP-6301-1097-L- } \\
\text { SP-6301-1097-L- } \\
\text { SP-6301-1097-L- } \\
\text { SP-6301-1097-L- } \\
\text { SP-6301-1097-L- } \\
\text { SP-6301-1097-L- } \\
\text { SP-6301-1097-L- } \\
\text { SP-6301-1097-L- } \\
\text { SP-6301-1097-L- } \\
\text { SP-6301-1097-L- } \\
\text { SP-6301-1097-L- } \\
\text { SP-6301-1097-L- } \\
\text { SP-6301-1097-L- } \\
\text { SP-6301-1097-L- } \\
\text { SP-6301-1097-L- } \\
\text { SP-6301-1097-L- } \\
\text { SP-6301-1097-L- } \\
\text { SP-6301-1097-L- } \\
\text { SP-6301-1097-L- } \\
\text { SP-6301-1097-L- } \\
\text { SP-6301-1097-L- } \\
\text { SP-6301-1097-L- } \\
\text { SP-6301-1097-L- } \\
\text { SP-6301-1097-L- } \\
\text { SP-6301-1097-L- } \\
\text { SP-6301-1097-L- } \\
\text { SP-6301-1097-L- } \\
\text { SP-6301-1097-L- } \\
\text { SP-6301-1097-L- } \\
\text { SP-6301-1097-L- } \\
\text { SP-6301-1097-L- } \\
\text { SP-6301-1097-L- } \\
\text { SP-6301-1097-L- } \\
\text { SP-6301-1097-L- } \\
\text { SP-6301-1097-L- } \\
\text { SP-6301-1097-L- } \\
\text { SP-6301-1097-L- } \\
\text { SP-6301-1097-L- } \\
\text { SP- } 6301-1097-L- \\
\text { SP-6301-1097-L- } \\
\text { SP-6301-1097-L- } \\
\text { SP-6301-1097-L- } \\
\text { SP-6301-1097-L- } \\
\text { SP-6301-1097-L- } \\
\text { SP-6301-1097-L- } \\
\text { SP-6301-1097-L- } \\
\text { SP-6301-1097-L- } \\
\text { SP-6301-1097-L- } \\
\text { SP-6301-1097-L- } \\
\text { SP-6301-1097-L- } \\
\text { SP-6301-1097-L- } \\
\text { SPP-6301-1097-L- } \\
\text { SP-6301-1097-L- } \\
\text { SP-6301-1097-L- } \\
\text { SP-6301-1097-L- } \\
\text { SP-6301-1097-L- } \\
\text { SP-6301-1097-L- } \\
\text { SP-6301-1097-L- } \\
\text { SP-6301-1097-L- } \\
\text { SP-6301-1097-L- }\end{array}$ & $\begin{array}{l}10 / 15 / 97 \\
10 / 15 / 97 \\
10 / 15 / 97 \\
10 / 15 / 97 \\
10 / 15 / 97 \\
10 / 15 / 97 \\
10 / 15 / 97 \\
10 / 15 / 97 \\
10 / 15 / 97 \\
10 / 15 / 97 \\
10 / 15 / 97 \\
10 / 15 / 97 \\
10 / 15 / 97 \\
10 / 15 / 97 \\
10 / 15 / 97 \\
10 / 15 / 97 \\
10 / 15 / 97 \\
10 / 15 / 97 \\
10 / 15 / 97 \\
10 / 15 / 97 \\
10 / 15 / 97 \\
10 / 15 / 97 \\
10 / 15 / 97 \\
10 / 15 / 97 \\
10 / 15 / 97 \\
10 / 15 / 97 \\
10 / 15 / 97 \\
10 / 15 / 97 \\
10 / 15 / 97 \\
10 / 15 / 97 \\
10 / 15 / 97 \\
10 / 15 / 97 \\
10 / 15 / 97 \\
10 / 15 / 97 \\
10 / 15 / 97 \\
10 / 15 / 97 \\
10 / 15 / 97 \\
10 / 15 / 97 \\
10 / 15 / 97 \\
10 / 15 / 97 \\
10 / 15 / 97 \\
10 / 15 / 97 \\
10 / 15 / 97 \\
10 / 15 / 97 \\
10 / 15 / 97 \\
10 / 15 / 97 \\
10 / 15 / 97 \\
10 / 15 / 97 \\
10 / 15 / 97 \\
10 / 15 / 97 \\
10 / 15 / 97 \\
10 / 15 / 97 \\
10 / 15 / 97 \\
10 / 15 / 97 \\
10 / 15 / 97 \\
10 / 15 / 97 \\
10 / 15 / 97 \\
10 / 15 / 97 \\
10 / 15 / 97 \\
10 / 15 / 97 \\
10 / 15 / 97\end{array}$ & 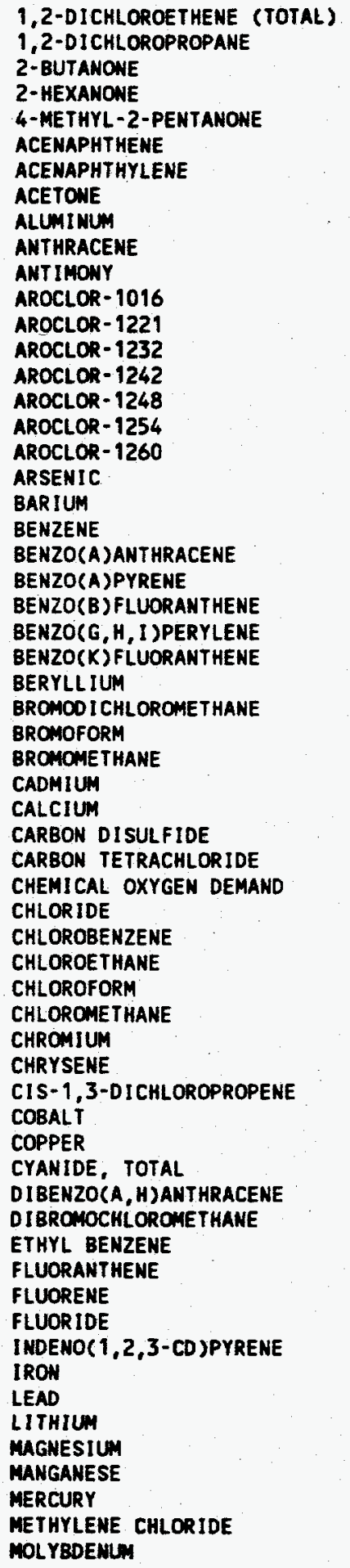 & 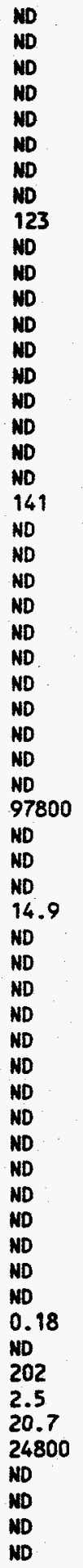 & 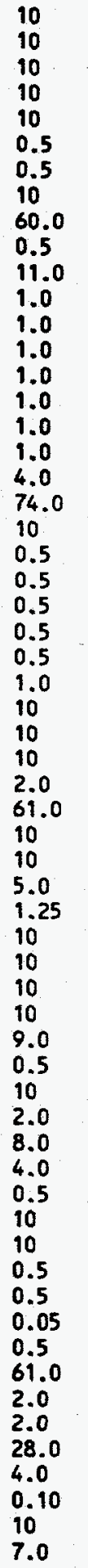 & 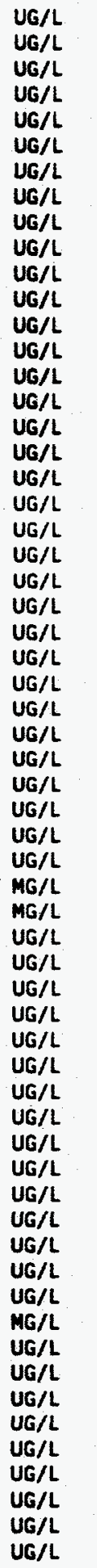 & 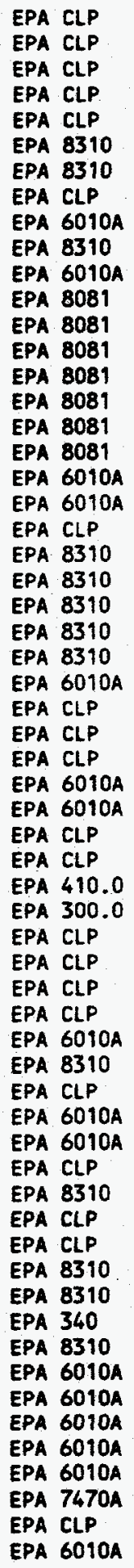 \\
\hline
\end{tabular}




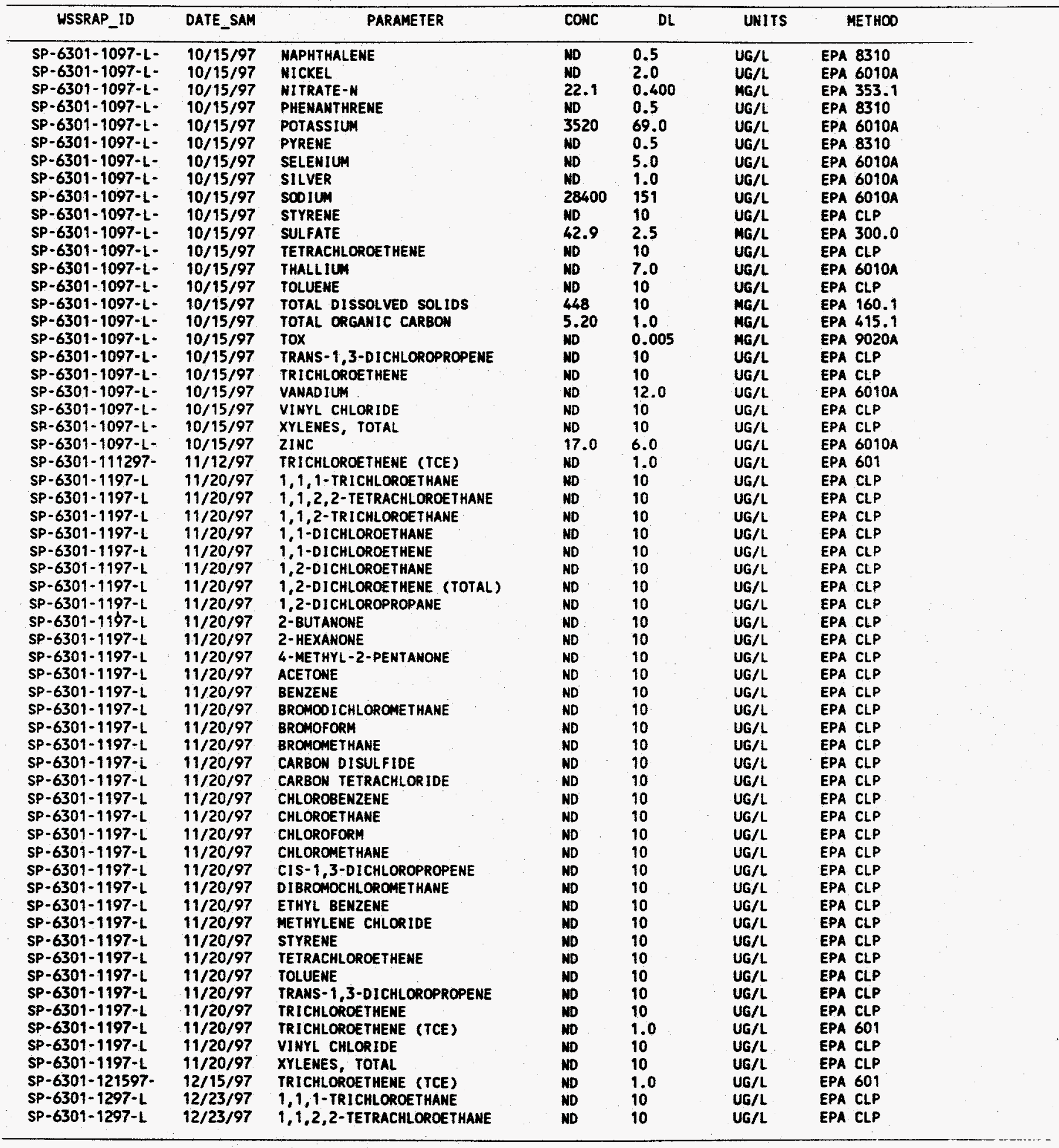




\begin{tabular}{|c|c|c|c|c|c|c|}
\hline WSSRAP_ID & DATE_SAM & PARAMETER & CONC & DL & UNITS & METHOD \\
\hline 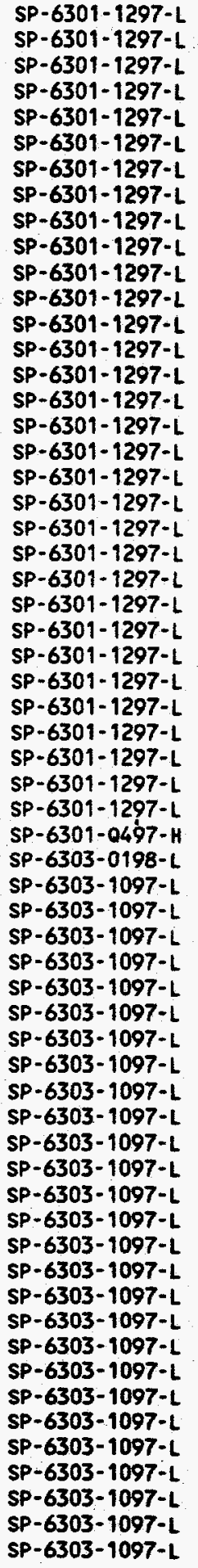 & $\begin{array}{l}12 / 23 / 97 \\
12 / 23 / 97 \\
12 / 23 / 97 \\
12 / 23 / 97 \\
12 / 23 / 97 \\
12 / 23 / 97 \\
12 / 23 / 97 \\
12 / 23 / 97 \\
12 / 23 / 97 \\
12 / 23 / 97 \\
12 / 23 / 97 \\
12 / 23 / 97 \\
12 / 23 / 97 \\
12 / 23 / 97 \\
12 / 23 / 97 \\
12 / 23 / 97 \\
12 / 23 / 97 \\
12 / 23 / 97 \\
12 / 23 / 97 \\
12 / 23 / 97 \\
12 / 23 / 97 \\
12 / 23 / 97 \\
12 / 23 / 97 \\
12 / 23 / 97 \\
12 / 23 / 97 \\
12 / 23 / 97 \\
12 / 23 / 97 \\
12 / 23 / 97 \\
12 / 23 / 97 \\
12 / 23 / 97 \\
12 / 23 / 97 \\
12 / 23 / 97 \\
11 / 06 / 97 \\
01 / 15 / 98 \\
10 / 29 / 97 \\
10 / 29 / 97 \\
10 / 29 / 97 \\
10 / 29 / 97 \\
10 / 29 / 97 \\
10 / 29 / 97 \\
10 / 29 / 97 \\
10 / 29 / 97 \\
10 / 29 / 97 \\
10 / 29 / 97 \\
10 / 29 / 97 \\
10 / 29 / 97 \\
10 / 29 / 97 \\
10 / 29 / 97 \\
10 / 29 / 97 \\
10 / 29 / 97 \\
10 / 29 / 97 \\
10 / 29 / 97 \\
10 / 29 / 97 \\
10 / 29 / 97 \\
10 / 29 / 97 \\
10 / 29 / 97 \\
10 / 29 / 97 \\
10 / 29 / 97 \\
10 / 29 / 97 \\
10 / 29 / 97 \\
10 / 29 / 97\end{array}$ & 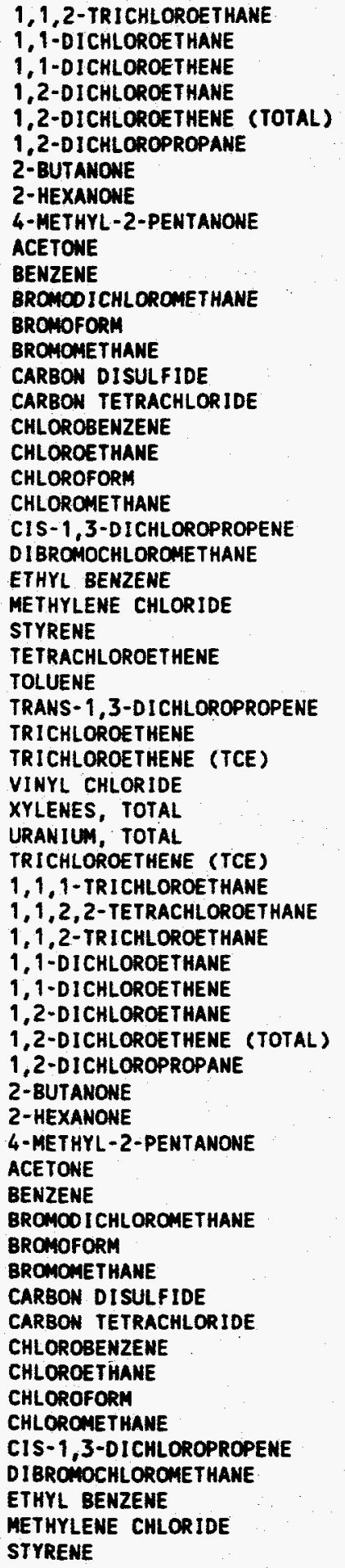 & $\begin{array}{l}\text { ND } \\
\text { ND } \\
\text { ND } \\
\text { ND } \\
\text { ND } \\
\text { ND } \\
\text { ND } \\
\text { ND } \\
\text { ND } \\
\text { ND } \\
\text { ND } \\
\text { ND } \\
\text { ND } \\
\text { ND } \\
\text { ND } \\
\text { ND } \\
\text { ND } \\
\text { ND } \\
\text { ND } \\
\text { ND } \\
\text { ND } \\
\text { ND } \\
\text { ND } \\
\text { ND } \\
\text { ND } \\
\text { ND } \\
\text { ND } \\
\text { ND } \\
\text { ND } \\
\text { ND } \\
\text { ND } \\
\text { ND } \\
27 \\
\text { CO.2) } \\
\text { ND } \\
\text { ND } \\
\text { ND } \\
\text { ND } \\
\text { ND } \\
\text { ND } \\
\text { ND } \\
\text { ND } \\
\text { ND } \\
\text { ND } \\
\text { ND } \\
\text { ND } \\
\text { ND } \\
\text { ND } \\
\text { ND } \\
\text { ND } \\
\text { ND } \\
\text { ND } \\
\text { ND } \\
\text { ND } \\
\text { ND } \\
\text { ND } \\
\text { ND } \\
\text { ND } \\
\text { ND } \\
\text { ND } \\
\text { ND }\end{array}$ & $\begin{array}{l}10 \\
10 \\
10 \\
10 \\
10 \\
10 \\
10 \\
10 \\
10 \\
10 \\
10 \\
10 \\
10 \\
10 \\
10 \\
10\end{array}$ & 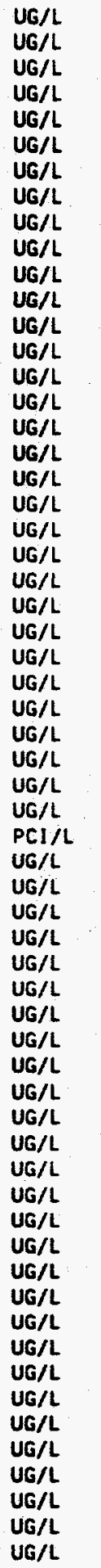 & 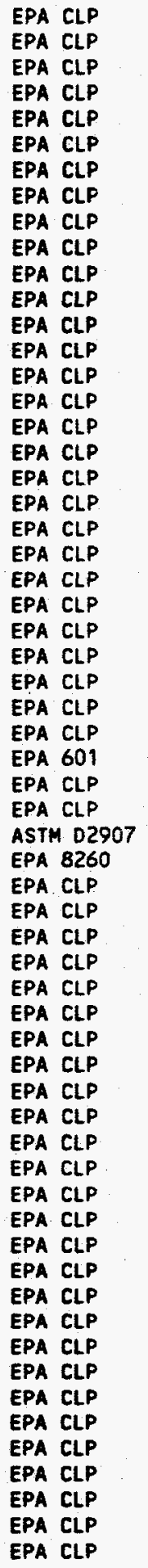 \\
\hline
\end{tabular}




\begin{tabular}{|c|c|c|c|c|c|c|}
\hline WSSRAP_ID & DATE_SAM & PARAMETER & CONC & $D L$ & UNITS & METHOO \\
\hline 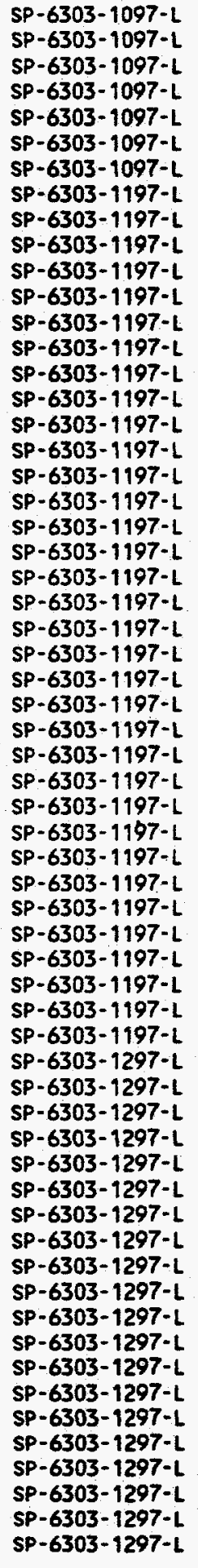 & $\begin{array}{l}10 / 29 / 97 \\
10 / 29 / 97 \\
10 / 29 / 97 \\
10 / 29 / 97 \\
10 / 29 / 97 \\
10 / 29 / 97 \\
10 / 29 / 97 \\
11 / 20 / 97 \\
11 / 20 / 97 \\
11 / 20 / 97 \\
11 / 20 / 97 \\
11 / 20 / 97 \\
11 / 20 / 97 \\
11 / 20 / 97 \\
11 / 20 / 97 \\
11 / 20 / 97 \\
11 / 20 / 97 \\
11 / 20 / 97 \\
11 / 20 / 97 \\
11 / 20 / 97 \\
11 / 20 / 97 \\
11 / 20 / 97 \\
11 / 20 / 97 \\
11 / 20 / 97 \\
11 / 20 / 97 \\
11 / 20 / 97 \\
11 / 20 / 97 \\
11 / 20 / 97 \\
11 / 20 / 97 \\
11 / 20 / 97 \\
11 / 20 / 97 \\
11 / 20 / 97 \\
11 / 20 / 97 \\
11 / 20 / 97 \\
11120 / 97 \\
11 / 20 / 97 \\
11 / 20 / 97 \\
11 / 20 / 97 \\
11 / 20 / 97 \\
11 / 20 / 97 \\
11 / 20 / 97 \\
12 / 23 / 97 \\
12 / 23 / 97 \\
12 / 23 / 97 \\
12 / 23 / 97 \\
12 / 23 / 97 \\
12 / 23 / 97 \\
12 / 23 / 97 \\
12 / 23 / 97 \\
12 / 23 / 97 \\
12 / 23 / 97 \\
12 / 23 / 97 \\
12 / 23 / 97 \\
12 / 23 / 97 \\
12 / 23 / 97 \\
12 / 23 / 97 \\
12 / 23 / 97 \\
12 / 23 / 97 \\
12 / 23 / 97 \\
12 / 23 / 97 \\
12 / 23 / 97 \\
\end{array}$ & 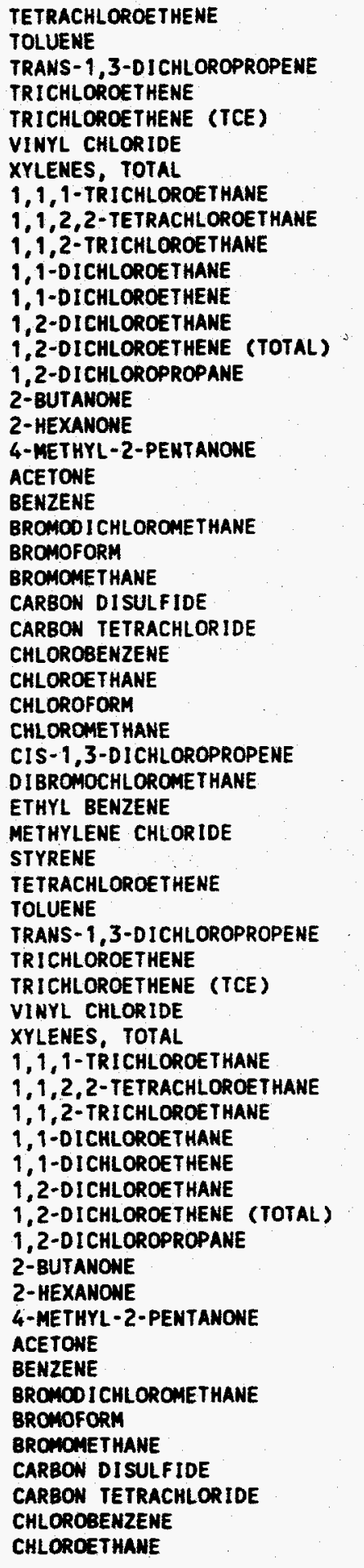 & $\begin{array}{l}\text { ND } \\
\text { ND } \\
\text { ND } \\
\text { ND } \\
\text { CD.35 } \\
\text { ND } \\
\text { ND } \\
\text { ND } \\
\text { ND } \\
\text { ND } \\
\text { ND } \\
\text { ND } \\
\text { ND } \\
\text { ND } \\
\text { ND } \\
\text { ND } \\
\text { ND } \\
\text { ND } \\
\text { ND } \\
\text { ND } \\
\text { ND } \\
\text { ND } \\
\text { ND } \\
\text { ND } \\
\text { ND } \\
\text { ND } \\
\text { ND } \\
\text { ND } \\
\text { ND } \\
\text { ND } \\
\text { ND } \\
\text { ND } \\
\text { ND } \\
\text { ND } \\
\text { ND } \\
\text { ND } \\
\text { ND } \\
\text { ND } \\
\text { ND } \\
\text { ND } \\
\text { ND } \\
\text { (2) } \\
\text { ND } \\
\text { ND } \\
\text { ND } \\
\text { ND } \\
\text { ND } \\
\text { ND } \\
\text { ND } \\
\text { ND } \\
\text { ND } \\
\text { ND } \\
\text { ND } \\
\text { ND } \\
\text { ND } \\
\text { ND } \\
\text { ND } \\
\text { ND } \\
\text { ND } \\
\text { ND } \\
\text { ND } \\
\text { ND } \\
\text { ND }\end{array}$ & $\begin{array}{l}10 \\
10 \\
10 \\
10 \\
1.0 \\
10 \\
10 \\
10 \\
10 \\
10 \\
10 \\
10 \\
10 \\
10 \\
10 \\
10 \\
10 \\
10 \\
10 \\
10 \\
10 \\
10 \\
10 \\
10 \\
10 \\
10 \\
10 \\
10 \\
10 \\
10 \\
10 \\
10 \\
10 \\
10 \\
10 \\
10 \\
10 \\
10 \\
1.0 \\
10 \\
10 \\
10 \\
10 \\
10 \\
10 \\
10 \\
10 \\
10 \\
10 \\
10 \\
10 \\
10 \\
10 \\
10 \\
10 \\
10 \\
10 \\
10 \\
10 \\
10 \\
10\end{array}$ & $\begin{array}{l}\text { UG/L } \\
U G / L \\
U G / L \\
U G / L \\
U G / L \\
U G / L \\
U G / L \\
U G / L \\
U G / L \\
U G / L \\
U G / L \\
U G / L \\
U G / L \\
U G / L \\
U G / L \\
U G / L \\
U G / L \\
U G / L \\
U G / L \\
U G / L \\
U G / L \\
U G / L \\
U G / L \\
U G / L \\
U G / L \\
U G / L \\
U G / L \\
U G / L \\
U G / L \\
U G / L \\
U G / L \\
U G / L \\
U G / L \\
U G / L \\
U G / L \\
U G / L \\
U G / L \\
U G / L \\
U G / L \\
U G / L \\
U G / L \\
U G / L \\
U G / L \\
U G / L \\
U G / L \\
U G / L \\
U G / L \\
U G / L \\
U G / L \\
U G / L \\
U G / L \\
U G / L \\
U G / L \\
U G / L \\
U G / L \\
U G / L \\
U G / L \\
U G / L \\
U G / L \\
U G / L \\
U G / L \\
\text { U. } \\
\text { U. }\end{array}$ & $\begin{array}{l}\text { EPA CLP } \\
\text { EPA CLP } \\
\text { EPA CLP } \\
\text { EPA CLP } \\
\text { EPA } 601 \\
\text { EPA CLP } \\
\text { EPA CLP } \\
\text { EPA CLP } \\
\text { EPA CLP } \\
\text { EPA CLP } \\
\text { EPA CLP } \\
\text { EPA CLP } \\
\text { EPA CLP } \\
\text { EPA CLP } \\
\text { EPA CLP } \\
\text { EPA CLP } \\
\text { EPA CLP } \\
\text { EPA CLP } \\
\text { EPA CLP } \\
\text { EPA CLP } \\
\text { EPA CLP } \\
\text { EPA CLP } \\
\text { EPA CLP } \\
\text { EPA CLP } \\
\text { EPA CLP } \\
\text { EPA CLP } \\
\text { EPA CLP } \\
\text { EPA CLP } \\
\text { EPA CLP } \\
\text { EPA CLP } \\
\text { EPA CLP } \\
\text { EPA CLP } \\
\text { EPA CLP } \\
\text { EPA CLP } \\
\text { EPA CLP } \\
\text { EPA CLP } \\
\text { EPA CLLP } \\
\text { EPA CLL } \\
\text { EPA } 601 \\
\text { EPA CLP } \\
\text { EPA CLLP } \\
\text { EPA CLP } \\
\text { EPA CLP } \\
\text { EPA CLP } \\
\text { EPA CLP } \\
\text { EPA CLP } \\
\text { EPA CLP } \\
\text { EPA CLP } \\
\text { EPA CLP } \\
\text { EPA CLP } \\
\text { EPA } ~ C L P ~ \\
\text { EPA CLP } \\
\text { EPA CLP } \\
\text { EPA CLP } \\
\text { EPA CLP } \\
\text { EPA CLP } \\
\text { EPA CLP } \\
\text { EPA CLP } \\
\text { EPA CLP } \\
\text { EPA CLP } \\
\text { EPA CLP }\end{array}$ \\
\hline
\end{tabular}




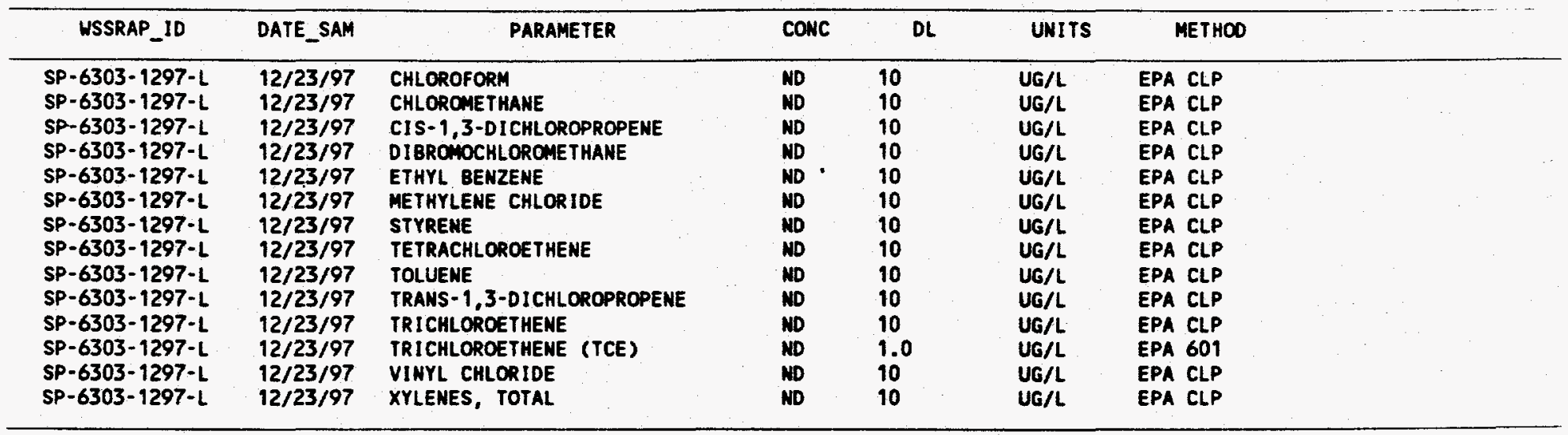


ting

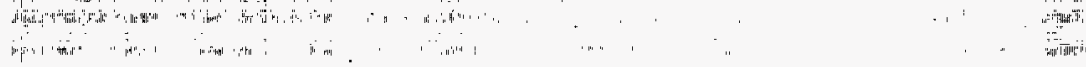

and

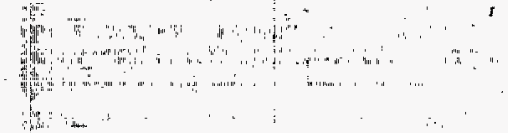

(1)

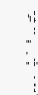

H.t.

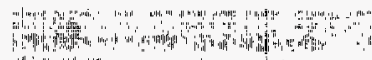

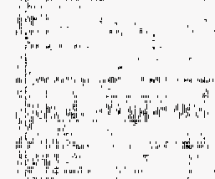

Hint

,

\section{AIR MONITORING}

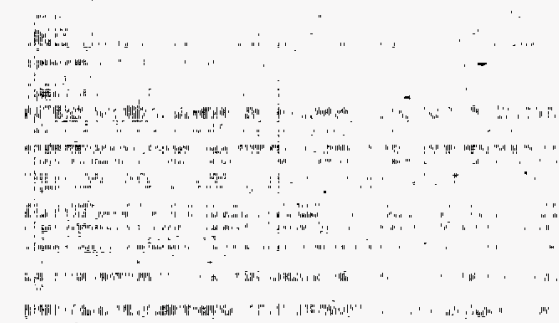

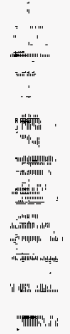

a

$=$

$\cdots$

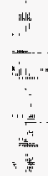

and

ind

"int

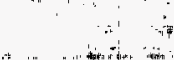

and

inti

n. $\cdots$

atin

a

- :i:

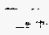

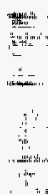




\section{First Quarter 1998 Air Particulate Results}

LOCATION ID
QUARTERLY AVERAGE GROSS

ALPHA CONCENTRATION (uCi/ml)
STANDARD

DEVIATION

$6.76 \mathrm{E}-16$

4.17E-16

3.55E-16

3.86E-16

$3.58 \mathrm{E}-16$

2.73E-16

$5.87 \mathrm{E}-16$

$4.50 \mathrm{E}-16$

3.71E-16

4.02E-16

$2.54 \mathrm{E}-16$

$3.89 \mathrm{E}-16$

3.92E-16

$3.34 \mathrm{E}-16$

$3.40 \mathrm{E}-16$

2.60E-16
NO. WEEKS

COLleCTED

AP-1017

$1.04 \mathrm{E}-15$

$1.02 \mathrm{E}-15$

* A new station installed in March

** Background monitoring location 
Air Particulate Concentration (uCi/ml)

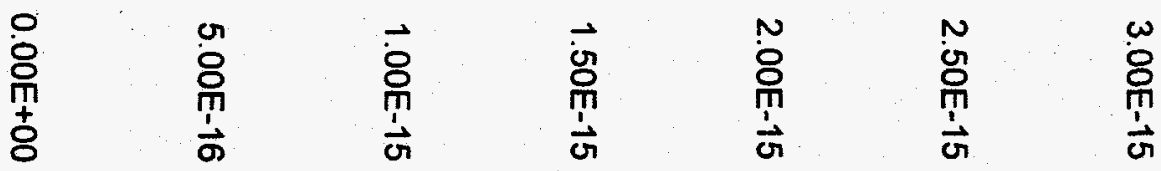

AP2001

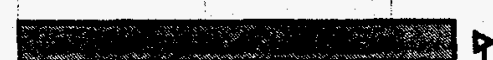

AP2002

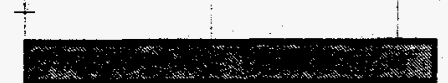

AP3003
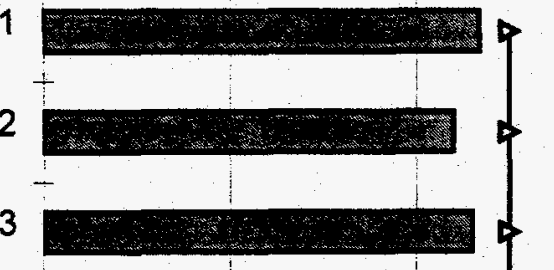

AP3004

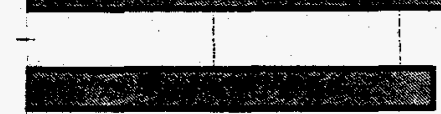

AP2005

$$
\div
$$

AP2008

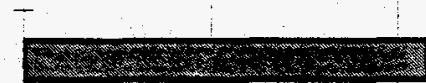

AP4006
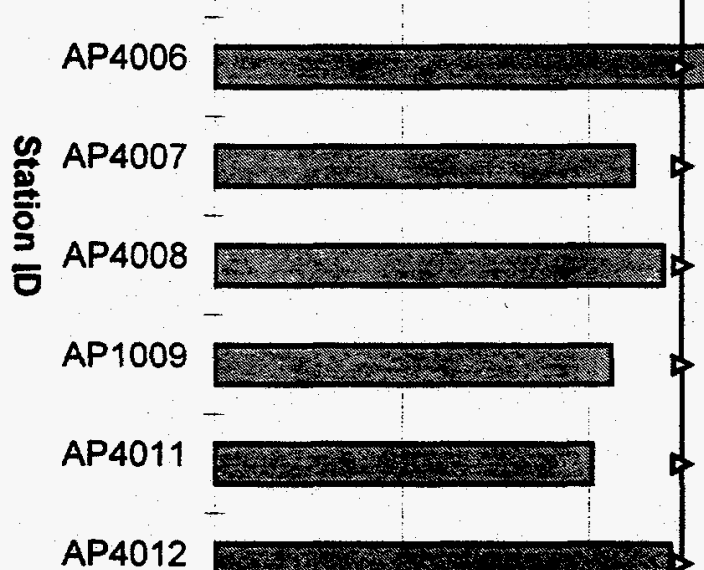

AP4012

AP4013

$-$

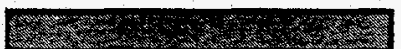

AP3014
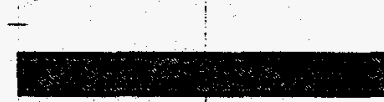


\section{Fourth Quarter 1997 Alpha Track Radon Monitoring Results}

LOCATION ID

$$
\text { AVERAGE }(\mathrm{pCi} / 1)^{@} \quad \text { PERCENT OF DCG }{ }^{\prime \prime}
$$

Weldon Spring Quarry

RD-1002

RD-1005

$\begin{array}{ll}0.4 & 0 \\ 0.4 & 0\end{array}$

Weldon Spring Chemical Plant

RD-2002

RD-2025

$\mathrm{RD}-2004$

$\mathrm{RD}-2006$

RD-2007

$\begin{array}{ll}0.4 & 0 \\ 0.4 & 0 \\ 0.3 & 0 \\ 0.3 & 0 \\ 0.5 & 3\end{array}$

Weldon Spring Raffinate Pits

RD-3001

RD-3002

$\mathrm{RD}-3003$

RD-3004

RD-3005

$\mathrm{RD}-3007$

$\mathrm{RD}-3008$

RD-3009

RD-3010

RD-3011

RD-3012

$\mathrm{RD}-3013$

RD-3014

$\mathrm{RD} \cdot 3015$

$\begin{array}{ll}0.7 & 10 \\ 0.7 & 10 \\ 1.0 & 20 \\ 0.4 & 0 \\ 0.5 & 3 \\ 6.5 & 203 \\ 0.7 & 10 \\ 0.5 & 3 \\ 2.4 & 67 \\ 0.6 & 7 \\ 1.0 & 20 \\ 2.3 & 63 \\ 1.0 & 20 \\ 0.9 & 17\end{array}$

Off-Site

$\begin{array}{lcc}\text { RD-4001 } & 0.3 & 0 \\ \text { RD-4002 } & 0.2 & 0 \\ \text { RD-4003 } & 0.2 & 0 \\ \text { RD-4005* } & 0.4 & \text { N/A } \\ \text { RD-4007 } & 0.4 & 0 \\ \text { RD-4009* } & 0.4 & \text { N/A } \\ \text { RD-4013 } & 0.2 & 0\end{array}$

* Denotes background location

N/A No percentage calculation performed on background locations

\# Derived Concentration Guide

@. Includes both radon and thoron 


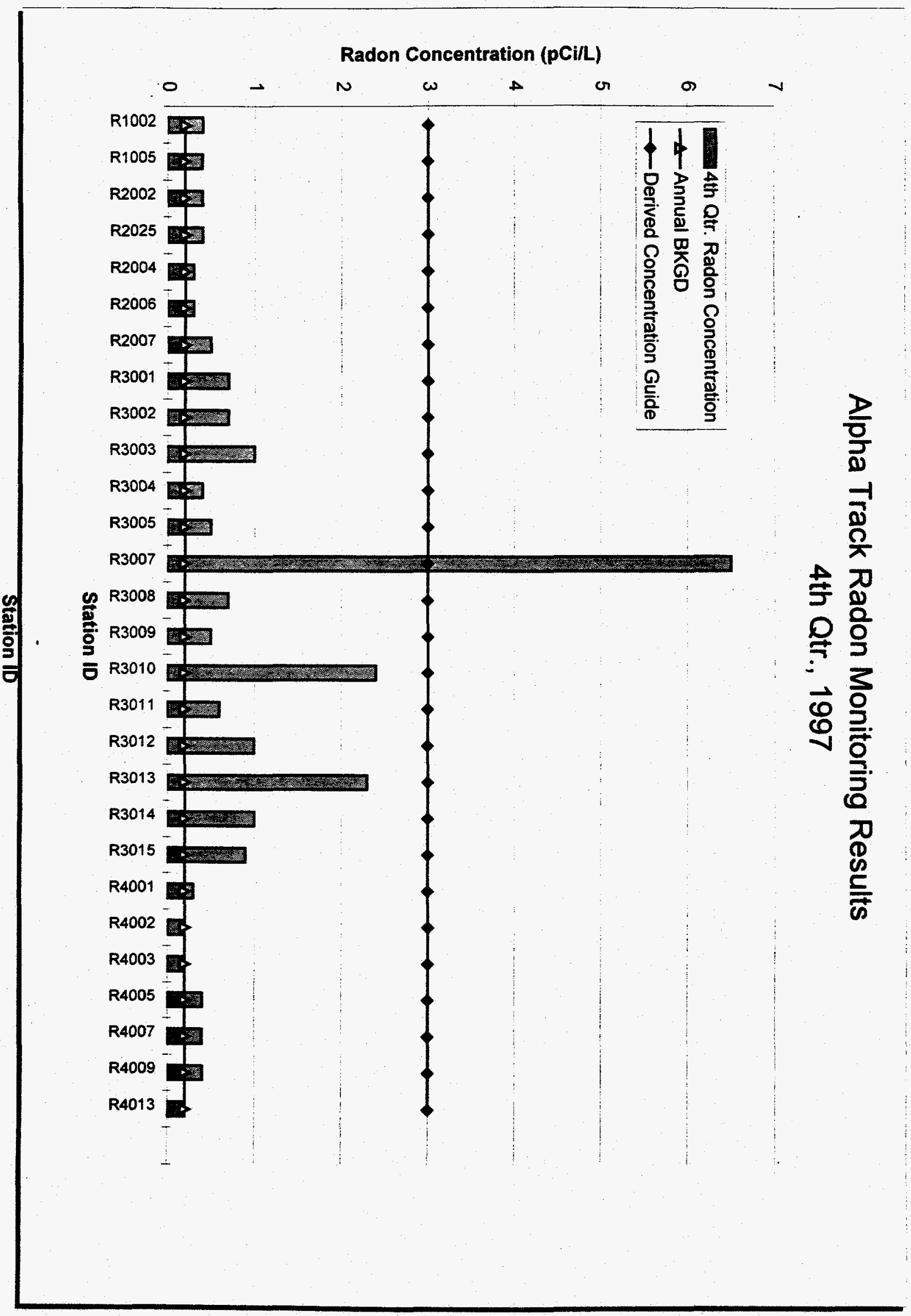




\section{Fourth Quarter 1997 Alpha Track Thoron Monitoring Results}

LOCATION ID

Weldon Spring Quarry

RD-1002

Weldon Spring Chemical Plant

RD-2002

RD-2025

$\mathrm{RD}-2004$

RD-2006

RD-2007

Weldon Spring Raffinate Pits

RD-3001

RD-3002

RD-3003

RD-3004

RD-3005

RD-3007

RD-3010

RD-3014

Off-Site

RD-4001

$\mathrm{RD}-4002$

RD 4003

RD-4005*

$\mathrm{RD}-4007$

RD-4009*

$\mathrm{RD}-4013$
AVERAGE(pCi $/ 1)$

0.3

0.3

0.3

0.2

0.0

0.3

$\begin{array}{ll}0.6 & 17 \\ 0.3 & 7 \\ 1.1 & 33 \\ 0.2 & 3 \\ 0.5 & 13 \\ 7.9 & 260 \\ 2.5 & 80 \\ 0.3 & 7\end{array}$

$\begin{array}{ll}0.2 & 3 \\ 0.0 & 0 \\ 0.0 & 0 \\ 0.0 & \text { N/A } \\ 0.2 & 3 \\ 0.2 & \text { N/A } \\ 0.1 & 0\end{array}$

* Denotes background location

N/A No percentage calculation performed on background locations

\# Derived Concentration Guide 


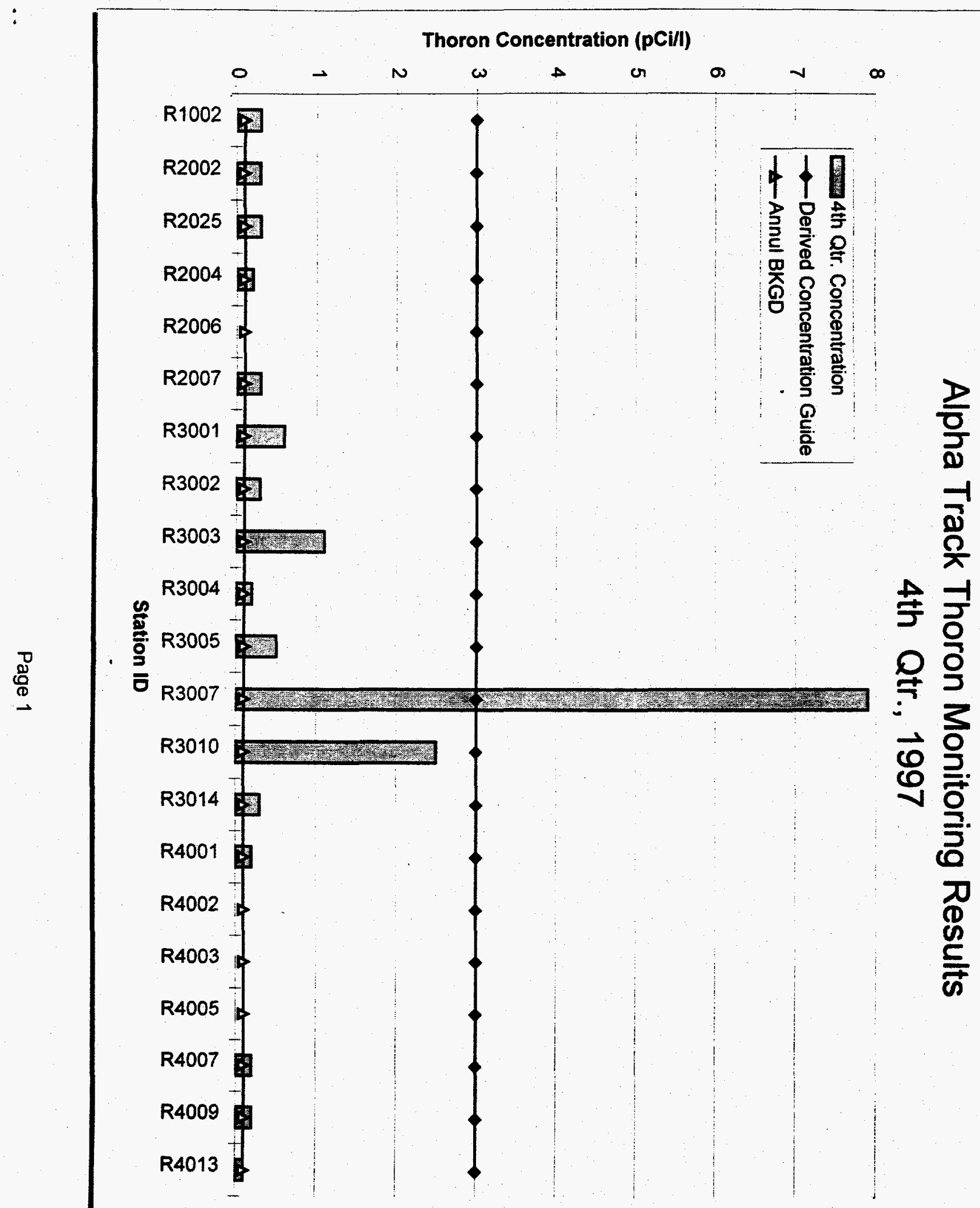




\title{
Fourth Quarter 1997 Environmental TLD Monitoring Results
}

LOCATION ID

\author{
GAMMA EXPOSURE
}

( $m R / Q t r)$

Weldon Spring Quarry

TD-1002

15.5

Weldon Spring Chemical Plant

TD-2025

TD-2004

TD-2006

17.7

18.3

15.8

Weldon Spring Raffinate Pits

TD-3001

TD-3002

TD-3003

TD-3004

17.6

19.5

20.0

19.6

Off-Site

TD-4001

TD-4002

TD-4003

TD.4005*

TD-4007

TD $-4009^{*}$

TD-4013

16.4

14.0

13.3

.14 .3

15.2

14.8

* Denotes background location

16.4

** Denotes the missing TLDs. 


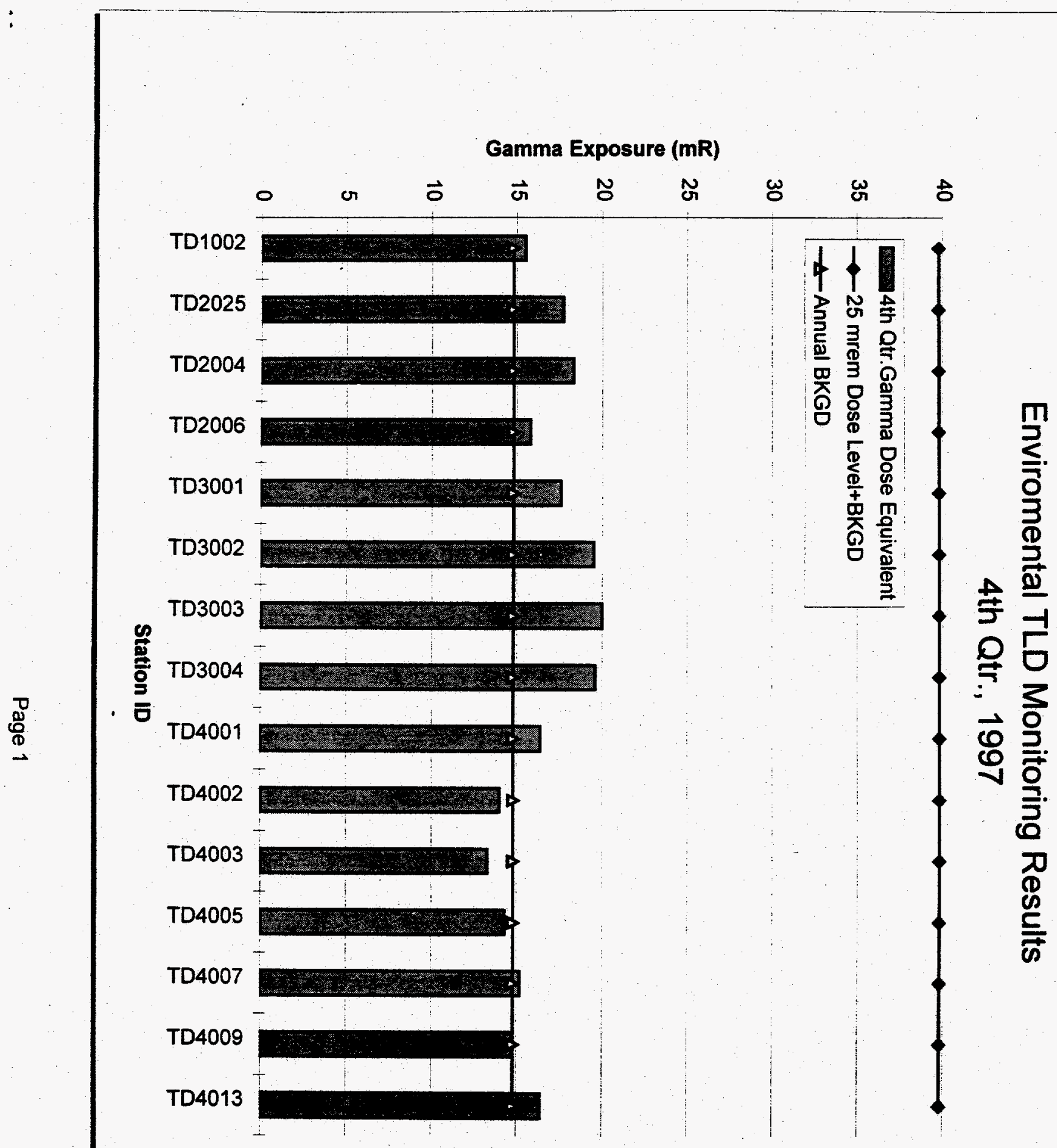

